University of Louisville

ThinkIR: The University of Louisville's Institutional Repository

Electronic Theses and Dissertations

$5-2021$

\title{
Anxiety and self-efficacy related to learning neuroanatomy in an integrated medical curriculum.
}

Jessica S. Bergden

University of Louisville

Follow this and additional works at: https://ir.library.louisville.edu/etd

Part of the Medical Education Commons

\section{Recommended Citation}

Bergden, Jessica S., "Anxiety and self-efficacy related to learning neuroanatomy in an integrated medical curriculum." (2021). Electronic Theses and Dissertations. Paper 3649.

https://doi.org/10.18297/etd/3649

This Doctoral Dissertation is brought to you for free and open access by ThinkIR: The University of Louisville's Institutional Repository. It has been accepted for inclusion in Electronic Theses and Dissertations by an authorized administrator of ThinkIR: The University of Louisville's Institutional Repository. This title appears here courtesy of the author, who has retained all other copyrights. For more information, please contact thinkir@louisville.edu. 


\title{
ANXIETY AND SELF-EFFICACY RELATED TO LEARNING NEUROANATOMY IN AN INTEGRATED MEDICAL CURRICULUM \\ By
}

\author{
Jessica S. Bergden \\ B.S., Purdue University, 1996 \\ M.S., University of Colorado Anschutz Medical Campus, 2015 \\ A dissertation submitted to the Faculty of the School of Medicine of the University of \\ Louisville in Partial Fulfillment of the Requirements for the Degree of
}

Doctor of Philosophy in Anatomical Sciences and Neurobiology

Department of Anatomical Sciences and Neurobiology,

University of Louisville,

Louisville, Kentucky

May 2021 

ANXIETY AND SELF-EFFICACY RELATED TO LEARNING NEUROANATOMY IN

AN INTEGRATED MEDICAL CURRICULUM

By

Jessica S. Bergden, M.S.

A Dissertation Approved on

April 16, 2021

By the following Dissertation Committee:

Jennifer Brueckner-Collins, Ph.D.

Dissertation Director

Nicole R. Herring, Ph.D.

Jason C. Immekus, Ph.D.

Erin M. Davis, Ph.D.

Robert F. Lundy, Jr. Ph.D. 


\section{DEDICATION}

This dissertation is dedicated to Dr. Robert Acland, whose innovation in medical education made this work possible. 


\section{ACKNOWLEDGMENTS}

I would like to acknowledge my mentor, Dr. Jennifer Brueckner-Collins. I could not have done this without your support.

A special thank you to my committee members: Dr. Jason Immekus for your patience, guidance, and high expectations that pushed me to challenge myself; Dr. Erin Davis for the countless supportive emails, calls, and texts that kept me going when I wanted to give up. Thank you for the time you devoted to this project; Dr. Nicole Herring for keeping me on task and setting the standard for dissection to which I aspire; and Dr. Robert Lundy for his neuroanatomy expertise and instructional direction.

To my family: Judi, Carl, Becky, and Jameson, for your love and support.

To all my friends who helped keep me sane during this process. Lisa, who sees my potential and pushes me to be the best version of myself. Jag vill tacka dig med hela mitt hjärta. Sanaya, who introduced me to a practice for which I will forever be grateful. You taught me my obstacles are only opportunities for growth. 


\section{ABSTRACT \\ ANXIETY AND SELF-EFFICACY RELATED TO LEARNING NEUROANATOMY IN AN INTEGRATED MEDICAL CURRICULUM}

Jessica S. Bergden

April 16, 2021

Medical student anxiety towards learning neuroanatomy — neurophobia, a key obstacle in medical education, is influencing medical student success and potentially swaying medical students away from neurology careers. This is particularly concerning with condensed neuroanatomy instructional hours from curriculum integration and the concurrent shortage of neurologists, combined with the rising prevalence of neurological disease, and medical students choosing neurological specialties at lower rates. Neuroanatomy education is one factor described in the literature as contributing to neurophobia, yet specific ways in which neuroanatomy education could be improved have not yet been explained. In this present work, we demonstrate four specific domains, namely content, instruction, communication, and organization, through which neuroanatomy education may be improved. We propose neuroanxiety may more accurately describe this phenomenon and developed a novel neuroanxiety scale. Our survey data show upper-class and female medical students exhibit greater neuroanxiety. Additionally, we demonstrate premedical neuroanatomy and/or neuroscience experience 
predicts a decrease in neuroanxiety. Another construct was explored as a way of potentially improving student task-specific confidence in neuroanatomy, namely — neuroanatomy self-efficacy. A novel neuroanatomy self-efficacy scale was developed and the effect of premedical neuroanatomy and/or neuroscience experiences on neuroanatomy self-efficacy was explored. Consistent with the literature on anatomy self-efficacy, our survey data revealed that premedical neuroanatomy experiences, especially with cadaveric dissection, improve neuroanatomy self-efficacy. Additionally, our data is trending towards female medical students showing a greater increase in neuroanatomy self-efficacy with premedical neuroscience and/or neuroanatomy exposure. Lastly, the exploration of developing a time-efficient learning intervention to be administered within an integrated curriculum led to the development of adaptive neuroanatomy eLearning intervention. Comparing the effects of the adaptive and non-adaptive eLearning interventions on medical student neuroanxiety and neuroanatomy self-efficacy demonstrated adaptive eLearning only significantly influenced neuroanatomy selfefficacy. This finding, together with our novel finding that premedical neuroanatomy and/or neuroscience experience also predicts lower neuroanxiety in medical school, suggests neuroanatomy self-efficacy may hold the key to mitigating the effects of neuroanxiety in neuroanatomy education. 
TABLE OF CONTENTS

PAGE

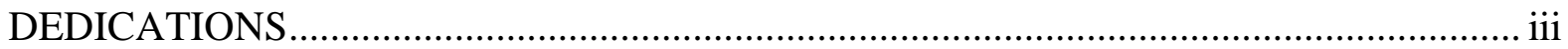

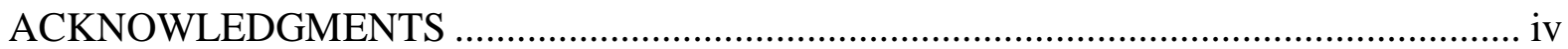

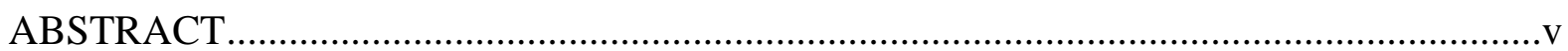

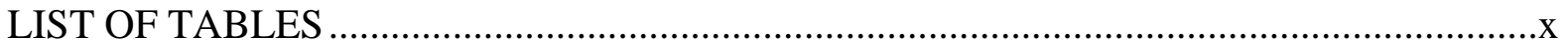

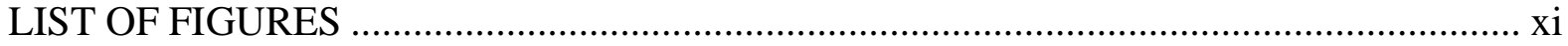

CHAPTER $\quad$ PAGE

CHAPTER 1 GENERAL INTRODUCTION ...............................................................

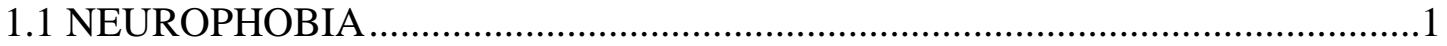

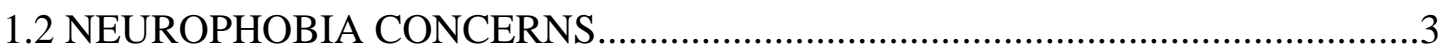

1.3 INSTRUCTIONAL CHALLENGES WITH NEUROPHOBIA ...........................

1.4 NEUROPHOBIA: PHOBIA OR ANXIETY? ...................................................

1.5 ACADEMIC SELF-EFFICACY AND NEUROANATOMY

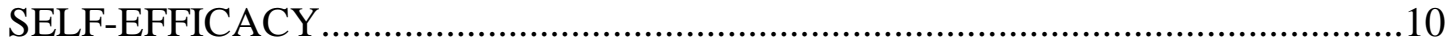

1.6 RELATIONSHIP BETWEEN SELF-EFFICACY AND ANXIETY ...................12

1.7 CURRICULUM INTEGRATION AND THE LOSS OF MEDICAL

NEUROANATOMY INSTRUCTIONAL HOURS ............................................. 14

1.8 DEVELOPING AN ADAPTIVE ELEARNING INTERVENTION ...................15

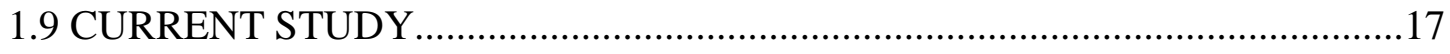

CHAPTER 2 MEDICAL STUDENTS AND NEUROPHOBIA: AN ESSENTIAL DISCUSSION PRIOR TO DEVELOPING AN ELEARNING INTERVENTION ..............20

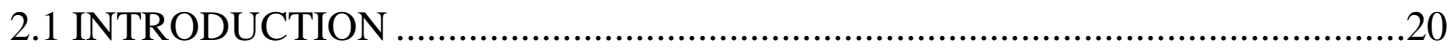

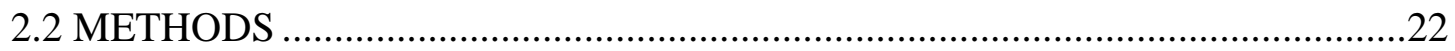

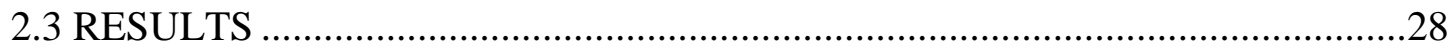

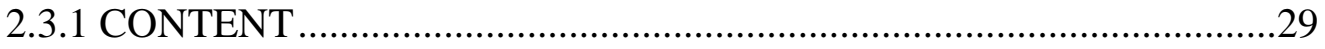

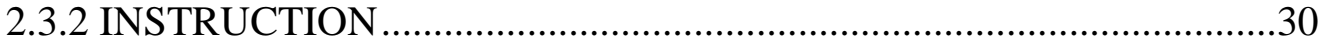

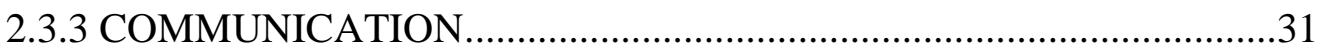

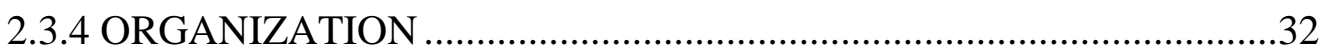

2.3.5 NEGATIVE EMOTIONAL RESPONSES .........................................33

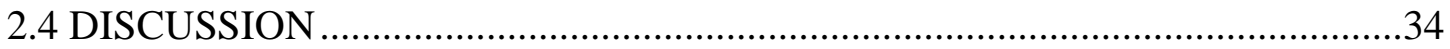

CHAPTER 3 PREDICTORS OF NEUROANATOMY ANXIETY (NEUROANXIETY)

AMONG MEDICAL STUDENTS IN AN INTEGRATED CURRICULUM......................48

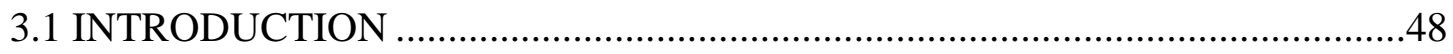


3.2 STUDY 1 NEUROANXIETY SCALE DEVELOPMENT AND INITIAL

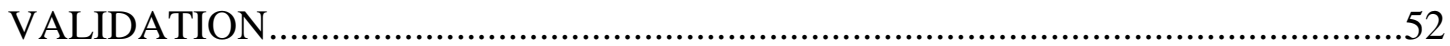

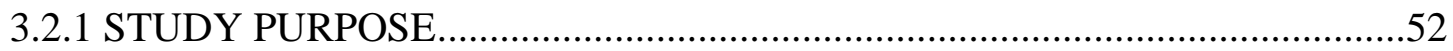

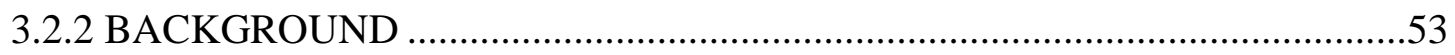

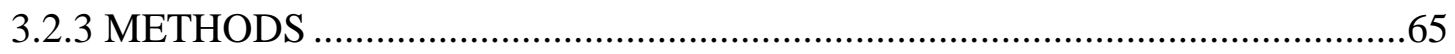

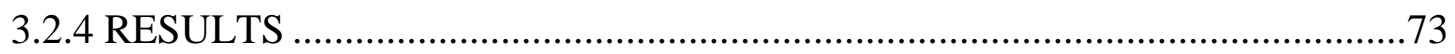

3.2.4.1 PARTICIPANTS AND RESPONSE RATE …………………...........73

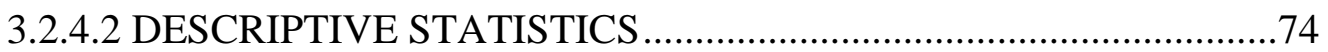

3.2.4.3 EXPLORATORY FACTOR ANALYSIS .......................................75

3.2.4.4 INTERNAL CONSISTENCY, VALIDITY, AND RELIABILITY ...76

3.2.4.5 NEUROANXIETY SCORES ………………...............................77

3.3 STUDY 2 PREDICTORS OF NEUROANXIETY ………................................

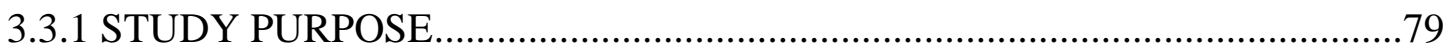

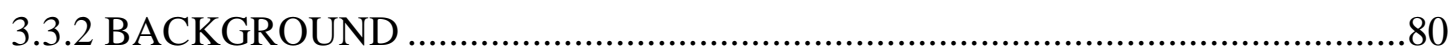

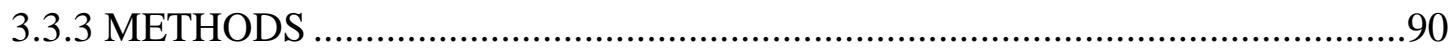

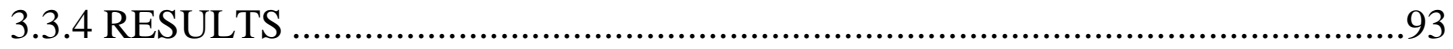

3.3.4.1 PREDICTORS OF NEUROANXIETY …………….......................93

3.5 OVERALL DISCUSSION …………………………...................................94

CHAPTER 4 EFFECTS OF PREMEDICAL NEUROANATOMY EXPERIENCES AND GENDER ON NEUROANATOMY SELF-EFFICACY IN AN INTEGRATED MEDICAL

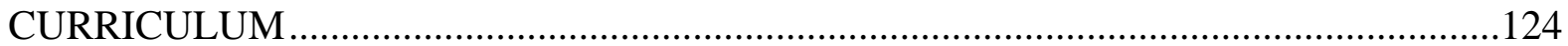

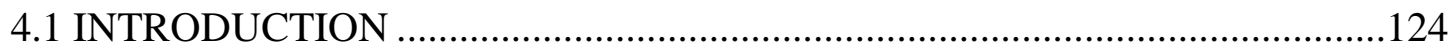

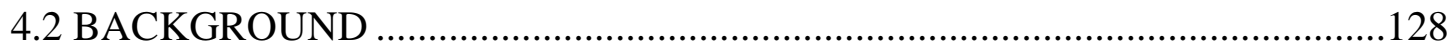

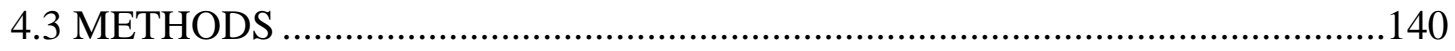

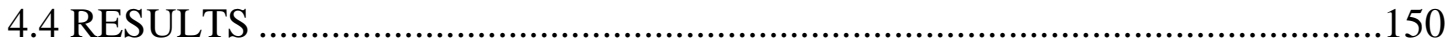

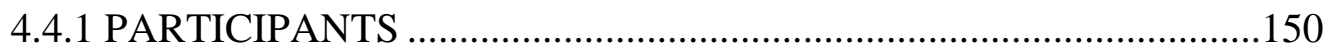

4.4.2 RESPONSE RATE AND DESCRIPTIVE STATISTICS ......................151

4.4.3 EXPLORATORY FACTOR ANALYSIS..........................................151

4.4.4 INTERNAL CONSISTENCY, VALIDITY, AND RELIABILITY ....154

4.4.5 NEUROANATOMY SELF-EFFICACY DIFFERENCES AND

PREMEDICAL NEUROSCIENCE AND/OR NEUROANATOMY

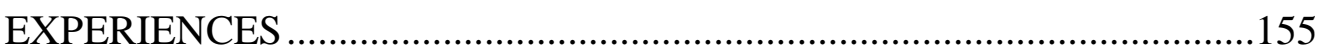

4.4.6 GENDER DIFFERENCES IN NEUROANATOMY SELF-EFFICACY AND PREMEDICAL NEUROSCIENCE AND NEUROANATOMY

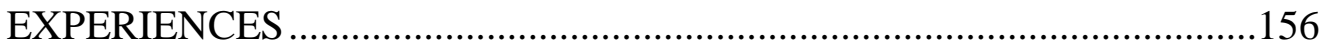

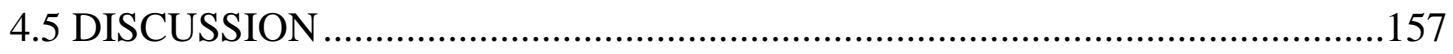

CHAPTER 5 COMPARING THE EFFECTS OF A NON-ADAPTIVE AND AN ADAPTIVE ELEARNING INTERVENTION ON FIRST-YEAR MEDICAL STUDENT NEUROANATOMY SELF-EFFICACY AND NEUROANXIETY IN AN INTEGRATED CURRICULUM.

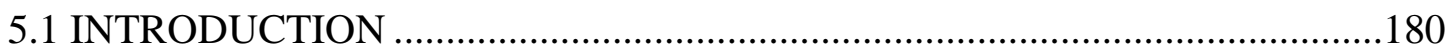

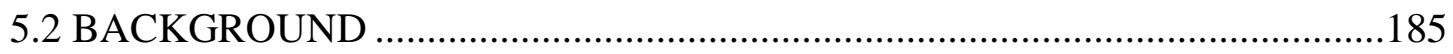

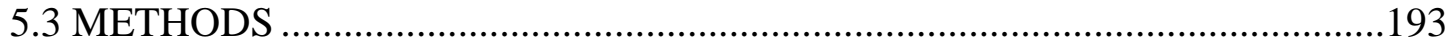

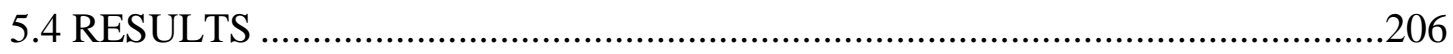


5.4.1 MODULE USAGE AND RESPONSE RATES ………………….......206

5.4.2 DESCRIPTIVE STATISTICS .........................................................207

5.4.3 NEUROANATOMY SELF-EFFICACY DIFFERENCES USING A NON-ADAPTIVE COMPARED TO AN ADAPTIVE NEUROANATOMY

ELEARNING INTERVENTION

5.4.4 NEUROANXIETY DIFFERENCES USING A NON-ADAPTIVE COMPARED TO AN ADAPTIVE NEUROANATOMY ELEARNING INTERVENTION

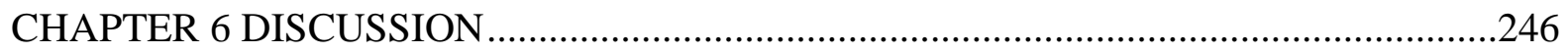

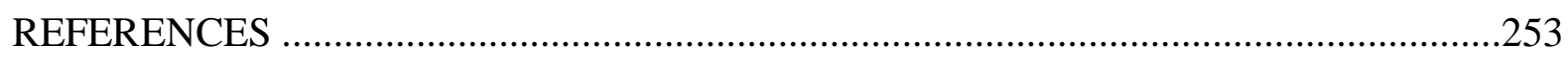

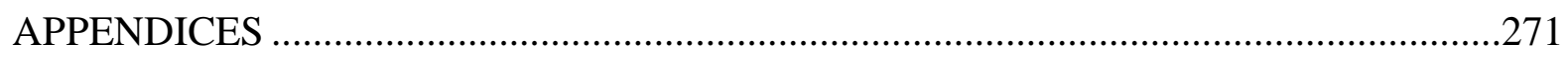

APPENDIX A ABBREVIATIONS...............................................................271

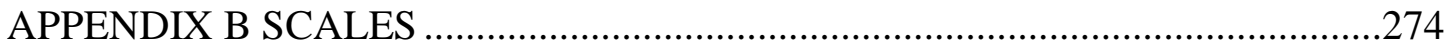

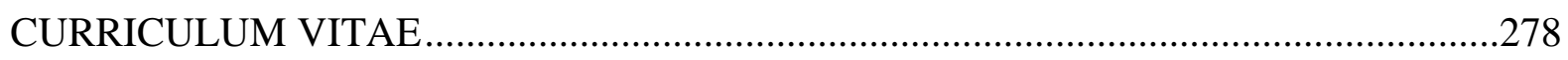




\section{LIST OF TABLES}

TABLE PAGE

1. TABLE 2.1

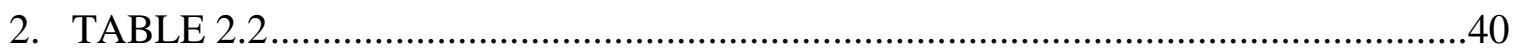

3. TABLE 2.3

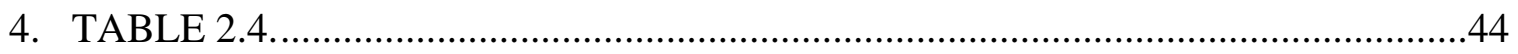

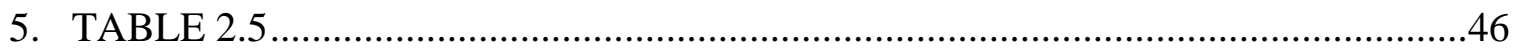

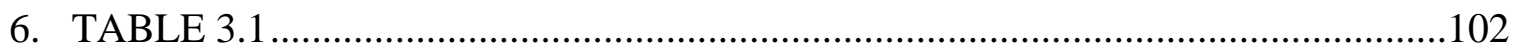

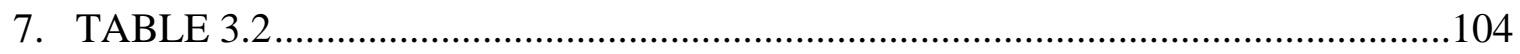

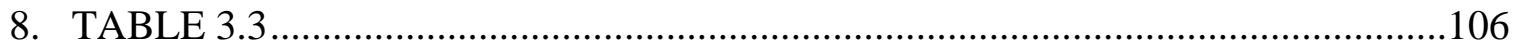

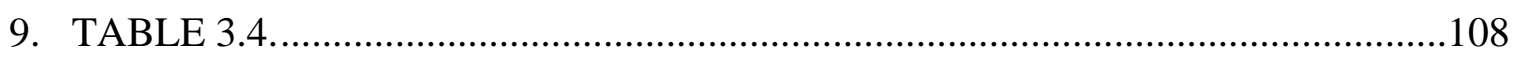

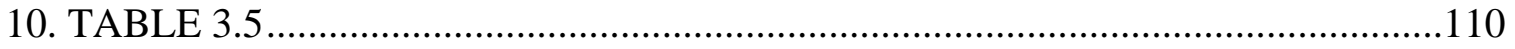

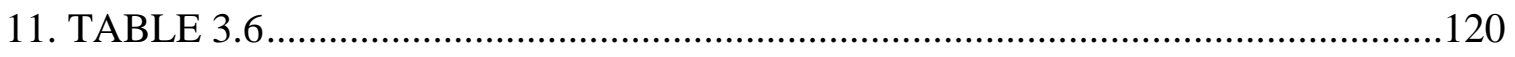

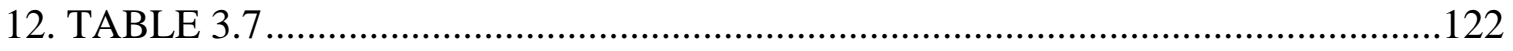

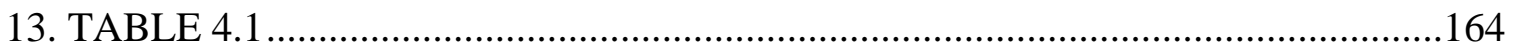

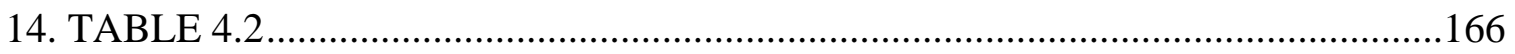

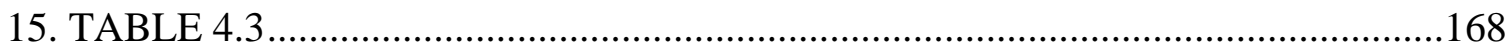

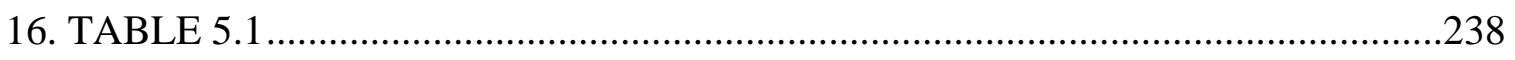

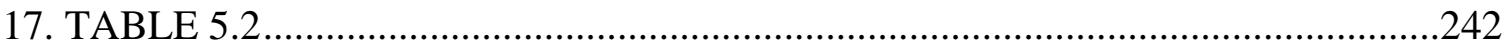




\section{LIST OF FIGURES}

FIGURE $\quad$ PAGE

1. FIGURE 3.1

2. FIGURE 3.2

3. FIGURE 3.3

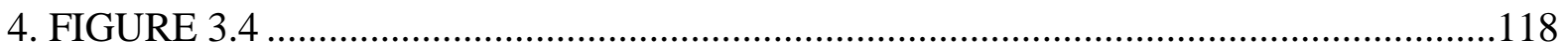

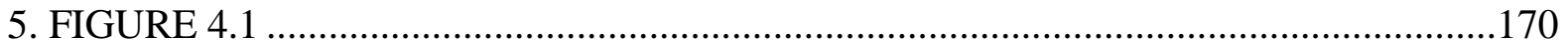

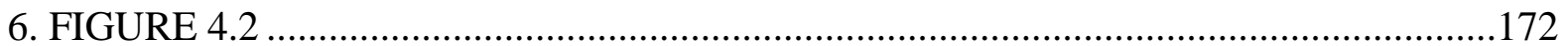

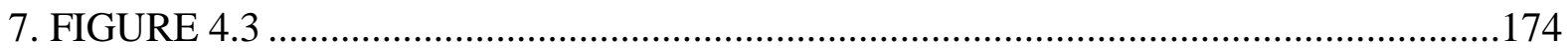

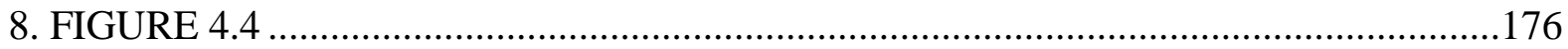

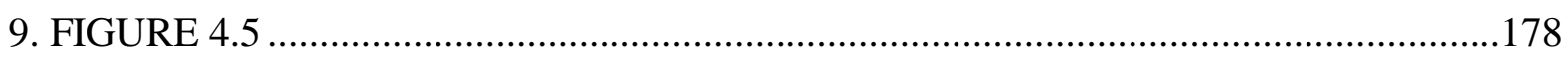

10. FIGURE 5.1

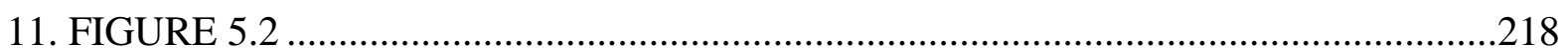

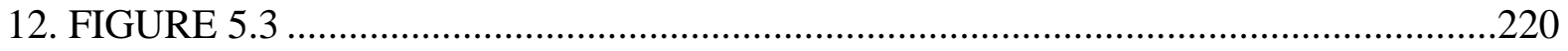

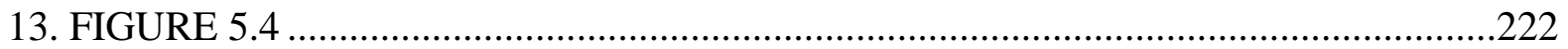

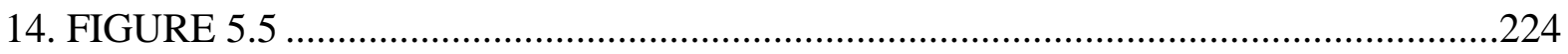

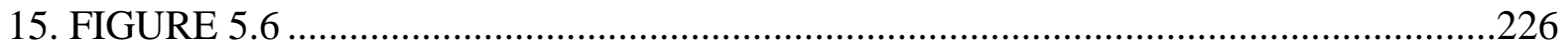

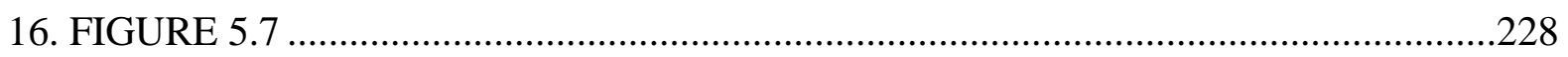

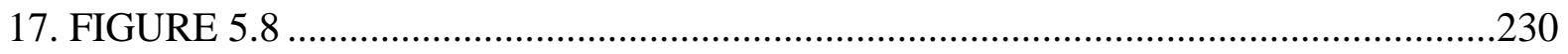

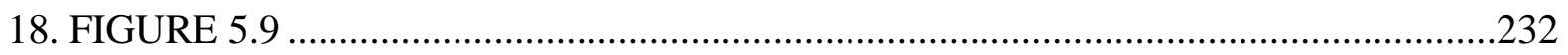

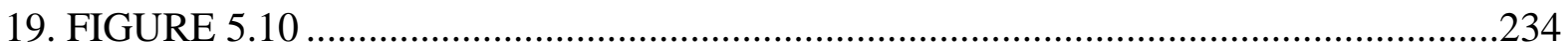




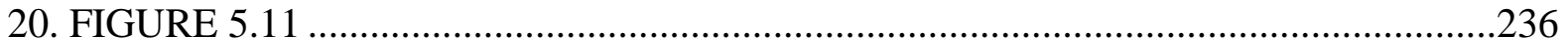

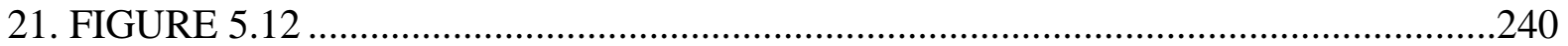

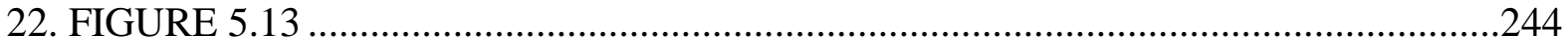




\section{CHAPTER 1}

\section{GENERAL INTRODUCTION}

1. 1 Neurophobia. Medical neuroanatomy is one of the most feared subjects by medical students. This fear of neuroanatomy in medical students led to the development of the term neurophobia, the debilitating "fear of the neural sciences and clinical neurology...due to the student's inability to apply their knowledge of basic sciences to clinical situations" (Jozefowicz, 1994, p. 328) in 1994. This neurophobia phenomenon has been widely documented to impact medical students worldwide in Australia (Hudson, 2006), the Caribbean (Youssef, 2009), India (Gupta et al., 2013), Ireland (Flanagan et al., 2007), Nigeria (Sanya et al., 2010), Saudi Arabia (Ablulaban et al., 2015), Sri Lanka (Matthias et al., 2013), the United Kingdom (Pakpoor et al., 2014), and the United States (Zinchuk et al., 2010). Neurophobia has even been documented, and on the rise, in practicing physicians as well (McCarron et al., 2014; Sandrone et al., 2019).

Neurophobia is a key issue in medical education and, as such, neurophobia will be explored in this dissertation. First, this general introduction will describe the major concerns surrounding neurophobia and why medical educators are investing time in exploring ways to minimize its effects. Next, instructional challenges with neurophobia will be highlighted. The study in Chapter 2 of this dissertation will add depth to the existing literature by exploring specific ways neuroanatomy instruction can be improved. 
The neurophobia construct itself will then be discussed and factors contributing to its development will be identified. Chapter 3 of this dissertation will explore the neurophobia construct, develop a scale to measure it, and contribute to the existing literature by investigating specific instructional predictors of neurophobia, informed from Chapter 2, in medical students. Additionally, another construct potentially related to anxiety called self-efficacy will be described. Chapter 4 of this dissertation will first explore academic self-efficacy and one of its main predictors, previous experience, and its relationship to medical student success. Subsequently, Chapter 4 will define a construct called neuroanatomy self-efficacy, develop a scale to measure neuroanatomy self-efficacy, and explore the effects of premedical neuroanatomy and/or neuroscience experience on neuroanatomy self-efficacy. Lastly, challenges of curriculum integration, namely the shortening neuroanatomy instructional hours in medical school, will be described and how decreased instructional hours may be influencing neurophobia. Chapter 5 of this dissertation describes the development of a time-efficient learning intervention that helps resolve the loss of neuroanatomy instructional hours. Additionally, the learning intervention described in Chapter 5, informed by the specific instructional strategies provided in Chapter 2, is designed to both reduce medical student neurophobia and improve neuroanatomy self-efficacy. The scales developed from Chapter 3 of this dissertation, measuring neurophobia, and Chapter 4, assessing neuroanatomy selfefficacy, will be utilized to explore the learning intervention's effects on both neurophobia and neuroanatomy self-efficacy in medical students. 
1.2 Neurophobia concerns. Currently, neurophobia is a major concern in medical education and clinical medicine, as a shortage of neurologists and neurosurgeons exists worldwide. This is alarming, as $6.4 \%$ of the world's health disease is due to neurological illness (World Health Organization, 2006), leading to a 12\% global mortality rate. These numbers are likely to rise due to the prevalence of neurological diseases, such as Alzheimer's disease and stroke later in life, combined with an aging world population (United Nations, 2002). Concurrently, a report by the American Academy of Neurologists (Freeman et al., 2013) already shows a present need for $11 \%$ more neurologists and this need is projected to rise to $19 \%$ by 2025 due to the aging population. The medical specialties of neurology, neuroradiology and neurosurgery are unable to meet the current patient demand for services. According to the American Association of Medical Colleges and American Medical Association (AMA, 2014), the neurosurgeon population alone has declined 4.5\% from 2008-2013 and more than 50.5\% of neurologists are 55 years and older. It is unknown if neurophobia is one reason for the decline in medical students choosing neurology as a specialty, however, it is an important consideration due to the shortage of specialists in the field.

Studies are underway to explore the declining interest in pursuing a neurology career and determine how to best attract medical students to the field. Gutmann et al. (2019) found the lack of work/life balance, salary expectations, personality fit, and poor clinical prognosis of neurology patients as reasons for medical students avoiding a career in neurology (Gutmann et al., 2019). Additionally, Gutman et al. (2019) found medical students who rated their pre-clinical neuroanatomy course as an excellent experience were $44 \%$ more likely to pursue a career in neurology compared to students who rated 
neuroanatomy as good. Students who reported strongly agree with clerkship neurology teaching effectiveness had a 50\% higher likelihood of entering neurology. Therefore, favorable teaching experiences, especially early in their medical education, may attract more students to the field. Further support for this relationship was demonstrated in a qualitative study investigating reasons behind medical students choosing neurology as a specialty by Gottlieb-Smith (2020). This study found class-wide burnout- due to the difficulty of the neurology course compressed into a short duration-as one reason for students shying away from a neurology career (Gottlieb-Smith, 2020). While favorable teaching experiences in neurology may attract students to neurology, alternately, unfavorable experiences may push students away from the field. These studies emphasize the importance of exploring which teaching experiences, perceived by medical students, improve overall learning in Chapter 2 of this dissertation. Additionally, the Gutman et al. (2019) study highlights the importance of early favorable neuroanatomy experiences on neurology specialty choice and stresses the importance of exploring neuroanatomy experiences of medical students before they enter medical school in Chapter 4 of this dissertation. These studies may add depth to the literature on how to attract more medical students to the field of neurology.

A second major concern with neurophobia in medical education is the gender disparity in neurological medicine with fewer female medical students pursuing careers in neurology, neurosurgery and neuroradiology. In a 2018 study surveying 1,712 academic neurologists, $30.8 \%(n=528)$ were women and $69.2 \%(n=1184)$ were men (McDermott et al., 2018). Men outnumbered women at all academic faculty ranks but the disparity grew with advancing rank, and only $13.8 \%$ neurology full professor 
appointments were women (McDermott et al., 2018). One may argue a gender disparity exists in medical school faculty departmental appointments in other specialties. A 2014 study from the Journal of the American Medical Association (JAMA) reports $11.9 \%$ of full professor appointments were women compared to $28.6 \%$ men in a cross-sectional database of 91,073 medical school faculty appointments- only a 16.7\% difference (Jena et al., 2015). While a gender disparity exists in many medical school faculty departmental appointments, a particularly large gender gap exists in neurological medicine.

Women specializing in neurology, neurosurgery, and neuroradiology earn disproportionately less than their male colleagues. The latest data from the American Academy of Neurologists (AAN) shows female academic neurologists earn $\$ 189,365$ compared with $\$ 250,000$ for men- a difference of $\$ 60,635$ (Fallik, 2016). The gender pay difference in the neurological specialties is particularly large when compared to other specialties. This is in contrast to a 2016 study from $J A M A$ demonstrating that the gender gap is closing when it comes to comparing the earning potentials academic physicians, among all specialties, at United States medical schools (Jena et al., 2016). Female full professors earn about the same $(\$ 247,212)$, on average, as male full professors $(\$ 250,971)$ at American medical schools. The gender pay gap may be one reason neurological specialties are less attractive for female medical students- the disparity in earning potential is larger in neurology compared to other specialties.

Addressing the factors contributing to the gender gap in physician numbers and salary are particularly important given that women are affected at higher rates for some long-term neurological diseases, (Multiple Sclerosis and Alzheimer's disease) (Zagni et al., 2016) yet there are fewer women neurologists to care for them. The American 
Academy of Neurology created a Gender Disparity Task Force (GDTF) to address the problem (AAN, 2017). Education is one focus of the GDTF since female medical students are choosing neurology $(5.4 \%$ vs $7 \%)$ and neurosurgery $(5.3 \%$ vs $7.3 \%)$ at a lower rate than their male counterparts (Ng-Sueng, 2016). Additional studies are investigating how to increase medical student recruitment (Lubelski et al., 2019), with a focus on women in order to narrow the gender gap in the field (LaFaver et al., 2018; Dixon et al., 2019; Hasan et al.; 2019). In a 2019 study to investigate female student retention in neurosurgery, Dixon et al. found $26.9 \%$ of female respondents considered a career in neurosurgery yet only $17.5 \%$ of matched neurosurgery residents in 2018 were women. Those female respondents were dissuaded from a neurosurgery career because they: felt they would face inequality and adversity that would prevent their success in a male-dominated field (26.9\%) and cited a lack of female neurosurgery mentors to mentor them $(88 \%)$ (Dixon et al., 2019). Increasing the number of female neurological specialists could help supplement the current shortage of neurologists and neurosurgeons in the United States while concurrently narrowing the gender gap. It is unknown whether neurophobia may be differentially affecting female medical students, thereby preventing them from pursuing a neurology career and is the rationale behind Chapter 3 of this dissertation exploring the gender differences in neurophobia.

1.3 Instructional challenges with neurophobia. Poor teaching is a consistent theme in neurophobia research (Flanagan et al, 2007; Youssef, 2009; Zinchuk et al., 2010; Abulaban et al., 2015). Flanagan et al. (2007) conducted a survey on neuroanatomy teaching administered to medical students $(n=457)$ and found respondents rated 
neuroanatomy teaching as moderate to poor on a 5-point Likert scale $(1=$ very poor to $5=$ very good). Another large-scale survey of medical student attitudes of neurology was conducted with 422 medical students at six different medical schools in Saudi Arabia (Abulaban et al., 2015). On a four-point Likert scale ( $1=$ least difficult to $4=$ most difficult), most (85.5\%) participants perceived neurology as difficult and $16 \%$ of respondents felt teachers were inadequate or needed improved teaching skills. Similarly, a survey of medical students and residents ( $n=152)$ (Zinchuk et al., 2010) reported poor neuroanatomy teaching in the first two years of medical school as contributing to neurophobia. This is concerning as most medical students (70.4\%) attribute their neurophobia to a bad teaching experience (Abulaban et al., 2015). However, the literature does not expand on the definition of poor teaching. Chapter 2 of this dissertation aims to address this literature gap by conducting a qualitative study exploring what specifically about neuroanatomy instruction improves or hinders medical student neuroanatomy learning. These instructional strategies learned from this study will inform the development of a learning intervention in Chapter 5 of this dissertation.

Investigating instructional methods and educational interventions designed to reduce neurophobia have been explored in the literature (Hudson et al., 2006; Flanagan et al., 2007; Anwar et al., 2015; Shiels et al., 2017; Javaid et al., 2018). Flanagan et al. (2007) conducted a survey to investigate which instructional methods may reduce neurophobia. Students were asked to rank 24 different instructional methods and suggested tutorials (29\%), greater exposure to neurology patients (11\%), video teaching (2.5\%), and improved online resources $(0.5 \%)$ were ways students perceived neuroanatomy teaching could be improved (Flanagan et al., 2007). Greater exposure to 
neurology patients has been explored in the literature through case-based teaching (CBT), team-based learning (TBL), and problem-based learning (PBL). Hudson et al. (2006) designed four 2-hour case-based tutorials (CBT) and assessed medical student $(n=119)$ performance on two neurological clinical examinations based on the CBT sessions. Of the students admitting to experiencing neurophobia (34\%), 20\% of these students described the CBT sessions as giving them more confidence in preparation for the clinical examination (Hudson et al., 2006). Anwar et al. (2015) used a causal comparative design to explore the effects of team-based learning (TBL) on neuroanatomy grades and neurophobia. Anwar et al. (2015) compared neuroanatomy grades of TBL students $(n=156)$ to neuroanatomy grades of the non-TBL students from the year prior and found TBL students performed better and fewer students overall were at lower risk of failing. Qualitative data was used to explore TBL on neurophobia and students reported TBL as beneficial in reducing their neurophobia (Anwar et al., 2015). Shiels et al. (2017) took a different approach and compared the effects of TBL, CBL, and PBL on first-year $(n=446)$ and second-year ( $n=206)$ medical student self-reported neurophobia. The authors found that CBT was the only instructional method that significantly reduced overall neurophobia (Shiels et al., 2017). Lastly, computer aided learning, or eLearning, was investigated in a separate study (Javaid et al., 2020). Medical students were either given an eLearning tool and didactic lecture $(n=40)$ or only didactic lecture $(n=36)$ to help isolate spinal cord and brainstem lesion problems. Students in the eLearning group reported greater assessment scores (Javaid et al., 2020) which suggests eLearning may benefit students learning neuroanatomy. 
The studies in this dissertation aim to contribute to the depth of this existing literature on instructional strategies to reduce neurophobia. Chapter 2 will add depth to specific instructional strategies to improve neuroanatomy learning and these strategies will be used to create an eLearning intervention in Chapter 5. Additionally, Chapter 3 will explore the instructional deficiencies, described by students in the qualitative study in Chapter 2, and their influence on neurophobia.

1.4 Neurophobia: Phobia or Anxiety? Critics of neurophobia have described this term as being "not totally serious" (Schon et al., 2002; p. 557), yet this phenomenon plagues our medical students. Neurophobia, if not being taken seriously, may be minimized by some in medical education despite its prevalence in medical students and physicians (Flanagan et al., 2007; Risdale et al., 2007; Youssef, 2009; Zinchuk et al., 2010; McCarron et al., 2014; Pakpoor et al., 2014; Javaid et al., 2018). This dissertation explores if there is a more accurate term that describes this neurophobia phenomenon. Jozefowicz defined neurophobia in 1994 as, "The debilitating fear of the neural sciences and clinical neurology...due to the student's inability to apply their knowledge of basic sciences to clinical situations" (p. 328). One medical student reported neurophobia elicited clinical levels of anxiety on a mental health diagnostic tool (Giles, 2010), the State Trait Anxiety Inventory (Spielberger et al., 1989). Even though this describes only a case study of one medical student, if neurophobia elicits anxiety on a published and validated anxiety disorder scale, one could hypothesize neurophobia may be more accurately classified as an anxiety and not a phobia. No other published psychological measures of anxiety or phobia were used to validate whether neurophobia was more 
appropriately categorized as a phobia or an anxiety. Chapter 3 of this dissertation will conceptualize neurophobia as neuroanxiety and develop an instrument designed to measure neuroanxiety levels in medical students.

1.5 Academic self-efficacy and neuroanatomy self-efficacy. Academic self-efficacy improves overall performance in medical school (Burgoon et al., 2012; Stegers-Jager et al., 2012; Hayat et al., 2020). Self-efficacy is defined as, "one's own perception of their ability to succeed at a task" (Bandura, 1977, p. 193) and the judgements one makes about their own abilities to complete tasks reflects their level of self-efficacy (Schunk, 1991). Self-efficacy is considered the critical agentic mechanism (Bandura, 1977), meaning one's belief in their ability to make things happen, and occupies a pivotal role in Social Cognitive Theory (SCT) (Bandura, 2001). In an academic setting, students with high selfefficacy believe they are capable of succeeding on examinations and have an influence on producing a desired outcome, such as receiving high scores. Medical students with increased self-efficacy performed better in the first year of medical school in gross anatomy (Burgoon et al., 2012) and on first-year final examinations (Stegers-Jager et al., 2012; Hayat et al., 2020). Additionally, medical students with higher self-efficacy scored higher on clinical performance examinations (Mavis, 2001) and self-efficacy predicted a medical student's clinical performance (Opacic, 2003). After graduation, medical residents with higher self-efficacy demonstrated improved patient care experiences (Young et al., 2012). Self-efficacy in relation to Social Cognitive Theory will be further explored further in Chapter 4 of this dissertation. 
Neuroanatomy specific self-efficacy has yet to be explored in the medical education literature. A definition of neuroanatomy self-efficacy, as proposed in Chapter 4, is based on Bandura's original definition of self-efficacy, "one's own perception of their ability to succeed at a task" (Bandura, 1977, p. 193). Chapter 4 proposes neuroanatomy self-efficacy will be defined as: One's perceived ability to successfully complete a neuroanatomical or clinical neurological task. In order to explore how to create an instrument to measure neuroanatomy self-efficacy, and create a learning intervention designed to improve neuroanatomy self-efficacy, one should consider the sources of self-efficacy, as described by Bandura (1977).

Bandura describes sources of self-efficacy as performance mastery, vicarious experience, verbal persuasion and emotional arousal (Bandura, 1977, 1986, 2001, 2012). Chapter 4 proposes these four sources of self-efficacy also contribute to neuroanatomy specific self-efficacy. Bandura (2001) explains that performance mastery creates the strongest level of self-efficacy through one's own experiences. Medical students performing, through independently successfully completing neuroanatomy questions, will successively build neuroanatomy self-efficacy. The specific questions to ask students must be organized according to difficulty level, however, asking easier questions before more difficult ones in order to accomplish building neuroanatomy self-efficacy. A second way of building self-efficacy, through vicarious experience, occurs through modeling one's thoughts or actions (Bandura, 2001). Modeling a thought process, especially the thought process required for challenging clinically-based lesion questions, will aim to increase neuroanatomy self-efficacy. It is also important for the instructor to remember how they felt when they were initially in the role of the student. Bandura (2001) proposed 
vicarious experience has more influence when modeled by someone who is similar to the student in emotional state. Not only modeling how to do the problem, but also modeling someone who is possibly feeling anxious about neuroanatomy and who overcame those feelings and succeeded in spite of their anxiety. Verbal persuasion is another source of self-efficacy and Bandura (2001) cautions it should be used minimally. However, consistent positive feedback while performing difficult neuroanatomy problems may provide medical students with some neuroanatomy self-efficacy. Lastly, perception of one's emotional arousal level is important on determining self-efficacy (Bandura, 2001). One student may perceive high emotional arousal (increased heart rate, breathing rate, sweating, etc...) as excitement, which may increase motivation and self-efficacy, while another student might perceive this emotional arousal as fear or anxiety. Possibly using words to describe complex neuroanatomy problems as exciting and energizing may increase neuroanatomy self-efficacy by framing emotional arousal towards excitement and away from anxiety. These instructional approaches are used to develop an eLearning module designed to increase neuroanatomy self-efficacy in Chapter 5 of this dissertation.

\subsection{The relationship between self-efficacy and anxiety. Emotional arousal, as}

described above, is one source of self-efficacy explained by Bandura (2001). How one perceives their level of emotional arousal may either improve, or diminish, one's selfefficacy (Bandura, 1997). As in the example above, one may perceive increased heart rate, breathing rate, and sweating as anxiety or as feeling energized and excited. This largely depends on how these emotions are interpreted. Bandura (2001) describes one aspect of Social Cognitive Theory called self-reactiveness, one's own self-reflection of 
their internal physiological state, has direct connections to the assessments one makes about their own emotions. Therefore, one medical student can feel emotional arousal (i.e. increased heart rate, breathing rate, sweating, etc...) and interpret this feeling as excitement which inspires a student to keep studying and learning through intentional action. Alternatively, another medical student may feel the same emotional arousal and perceive this emotion as anxiety which overwhelms the student and demotivates study. Anxiety and self-efficacy are related through one's emotional arousal level and concurrent perception of this emotional arousal. One may theorize that neuroanatomy self-efficacy and neuroanatomy anxiety have a similar relationship. This hypothesized relationship between self-efficacy and anxiety through Social Cognitive Theory provides the rationale behind designing the eLearning intervention to target both neuroanxiety and neuroanatomy self-efficacy, and will be further described, in Chapter 5 of this dissertation.

Few studies suggest the potential of self-efficacy to moderate (decrease) educational anxiety. One study (Barrows et al., 2013) of college students ( $n=110)$ measured both student text anxiety and self-efficacy three days prior and immediately after an examination. Barrows et al. (2013) found self-efficacy did not decrease test anxiety for a single college examination. However, students with higher self-efficacy and lower anxiety scored higher on the examination than those students with lower selfefficacy and higher anxiety. No published studies to-date were found comparing medical student neuroanatomy self-efficacy and anxiety in neuroanatomy. Medical students have the documented perception that neuroanatomy is an anxiety-inducing subject because of its difficulty level (Schon et al., 2002; Flanagan et al., 2007; Youssef, 2009; Zinchuk et 
al., 2010; McCarron et al., 2014; Abulaban et al., 2015). Improving neuroanatomy selfefficacy may be more effective than attempting to change student's perception about neuroanatomy, or even their neuroanxiety level. Chapter 5 aims to fill that gap in the literature by measuring the effect of an eLearning intervention designed to reduce neuroanxiety and increase neuroanatomy self-efficacy of medical students.

\subsection{Curriculum integration and loss of medical neuroanatomy instructional hours.}

Recent curriculum change in medical education has transitioned a systems-based approach to a clinically integrated approach (Muller et al., 2008; Drake et al., 2009; Chen et al., 2014; Gonzalo et al., 2017). The new clinically integrated approach requires decreasing medical school instruction hours (Drake et al., 2009; McBride \& Drake, 2018; Choi-Lundberg et al., 2019; Selvarajah \& Aojula, 2019; Hannan et al., 2020), favors competency-based education over traditional grading systems (Berwick et al., 2010; Frenk et al., 2010; Combes \& Arespacochaga, 2012), and a collaborative team-based approach over independent physician problem-solving (Lucey, 2013). An integrated curriculum presents basic science information alongside relevant clinical applications throughout every step of a student's medical education. Brauer and Ferguson (2015) defined three ways in which medical curricula can be integrated: horizontal, vertical, and spiral. Horizontal integration merges subjects within a specific timeframe. For example, instead of teaching gross anatomy, histology, neuroanatomy and clinical skills separately in their first year of medical school- one integrated first year course replaces these by presenting the gross anatomy of the muscles concurrently with their histology, innervation and clinical muscle testing on a patient. Vertical integration is presentation of 
material across all four years of medical education. In practice, this is demonstrated by exposure to clinical applications early in the curriculum and testing of basic science information later in the curriculum. A fully integrated medical curriculum is a spiral model consisting of both horizontal and vertical types of integration. The new framework of a fully integrated curriculum aims to prepare students for the real-life practice of medicine (Drake et al., 2002; Price, 2005; Gonzalo et al., 2017).

However, one weakness of curriculum integration has been the loss of neuroanatomy instructional hours. Mateen and D'Eon (2008) describe neuroanatomy as being "increasingly marginalized in medical school curricula" (p. 538). Neuroanatomy suffered the largest drop- $18 \%$ decrease in lecture and laboratory hours in a survey of medical schools ( $n=84$ ) when converting the curriculum from systems-based to an integrated approach (Drake et al., 2002; Drake et al. 2009). Concurrently, medical students are experiencing neurophobia at increasing rates (Schon et al., 2002; Flanagan et al., 2007; Youssef, 2009; Zinchuk et al., 2010; McCarron et al., 2014; Abulaban et al., 2015). The combined loss of neuroanatomy instructional hours and increasing medical student anxiety towards learning neuroanatomy in medical education is an obstacle needing attention. Chapter 5 of this dissertation aims to address this challenge by providing a supplemental neuroanatomy instructional tool, for use within an integrated curriculum, designed to reduce neuroanxiety.

\subsection{Developing an adaptive eLearning intervention. Developing a supplemental} neuroanatomy eLearning intervention may be an innovative solution as both neuroanatomy instructional hours are decreasing (Drake et al., 2002; Drake et al. 2009) 
and students are demonstrating increased anxiety towards learning neuroanatomy (Schon et al., 2002; Flanagan et al., 2007; Youssef, 2009; Zinchuk et al., 2010; McCarron et al., 2014; Abulaban et al., 2015). There is an increasing trend of implementing adaptive eLearning in medical education because adaptive eLearning may decrease instructional time while concurrently increase accuracy and student performance (Krasne et al., 2013; Hu et al., 2016; Romito et al., 2016; Samulski et al., 2018). Adaptive eLearning forces students to demonstrate stepwise competencies- where students progress easily through competent areas while additional content is provided for those areas where students struggle. High performing students can quickly progress through an adaptive eLearning module in a time efficient manner by demonstrating competency. Additionally, lower performing students will receive the extra information they need to understand the subject, prior to subsequently demonstrating competency and completing the module.

Research studies show medical students perceived neuroanatomy eLearning as beneficial (Foreman et al., 2005; Svirko and Mellanby, 2017). In one study, $88 \%$ of students found eLearning beneficial overall with $95 \%$ of students rating eLearning as better than traditional learning tools (Foreman et al., 2005). A 2017 study examined the depth of learning in second year medical students' $(n=869)$ approach to neuroanatomy; deep learning was defined as being motivated by subject matter interest and alternatively, surface learning was defined as being motivated by fear of failure (Svirko \& Mellanby, 2017). This study found eLearning was positively correlated with deep learning; concurrently, a positive correlation between deep learning and academic performance was reported $(r=0.12, p<0.001)$ (Svirko \& Mellanby, 2017). The development of an adaptive eLearning intervention may decrease overall instructional time and be more time 
effective for the individual student. In summary, the literature suggests neuroanatomy eLearning is perceived as beneficial by medical students while promoting deep learning with improved medical student outcomes. This is the rationale behind creating the adaptive eLearning intervention, for use in an integrated curriculum, in Chapter 5 of this dissertation.

1.9 Current study. Four studies are proposed in light of the current challenges that exist in neuroanatomy education with a concurrent neurology shortage and a gender disparity in neurological medicine. These studies will explore the constructs of neurophobia and neuroanatomy self-efficacy, provide scales to measure them, and assess an eLearning intervention designed to mitigate neurophobia and improve neuroanatomy self-efficacy among first-year medical students.

The first qualitative study will explore medical student attitudes towards neuroanatomy instruction to both identify the presence of neurophobia at our institution and inform the development of eLearning interventions aimed to target neurophobia. The second study will explore neurophobia and aims to develop a novel scale to measure it. One limitation of published neurophobia research is the lack of a validated scale to measure it. Much of the published neurophobia research (Flanagan et al., 2007; Youssef, 2009; Zinchuk et al., 2010; McCarron et al., 2014; Abulaban et al., 2015) uses a survey derived from a single scale (Schon et al., 2002). These survey items compare neurology with other medical specialties, yet do not measure neurophobia itself. An operational definition of a latent construct is vital in order to create an instrument to adequately measure it, as described by Standard 1.1 from the Standards in Educational \& 
Psychological Measurement: “...the construct or constructs that the test is intended to assess should be clearly described" (American Educational Research Association et al., 2014). Development of a novel neurophobia scale and initial scale validation will be explored. Additionally, gender differences in neurophobia will be explored in light of fewer women choosing a career in neurological specialties (Zagni et al., 2016). The third study will investigate the construct of neuroanatomy self-efficacy. Neuroanatomy selfefficacy has yet to be explored in the literature and this study will fill this gap by providing a theoretical framework for neuroanatomy self-efficacy, develop a novel instrument to measure it, and provide data for initial validity and reliability evidence for the instrument. Additionally, the effect of premedical neuroscience and/or neuroanatomy experiences on neuroanatomy self-efficacy will be explored. The final study will investigate the effects of an eLearning intervention on neurophobia and neuroanatomy self-efficacy levels of first-year medical students utilizing the newly developed scales in the second and third studies. The aims of the four studies are highlighted below:

(1) This study investigates first-year medical students' perceptions of neuroanatomy in order to investigate the presence of neurophobia at our institution and explore instructional strategies that may lead to, or alternately reduce, neurophobia. Specific instructional strategies, provided by qualitative student responses in this study, will be used to develop an eLearning intervention designed to strategically mitigate neurophobia in the fourth study.

(2) An initial neurophobia scale development study will seek to: (a) measure the construct of neurophobia to obtain psychometric properties for initial validation; 
(b) investigate predictor variables of neurophobia; and (c) provide a measure to study the efficacy of a learning intervention designed to mitigate neurophobia.

(3) An initial neuroanatomy self-efficacy scale development study will seek to: (a) measure the construct of neuroanatomy self-efficacy to obtain psychometric properties for initial validation; (b) investigate the effect of gender and premedical neuroanatomy experiences on neuroanatomy self-efficacy; and (c) provide a measure to study the effectiveness of a learning intervention designed to improve neuroanatomy self-efficacy.

(4) This study will aim to: (a) design an eLearning intervention to both reduce neurophobia and increase neuroanatomy self-efficacy; and (b) investigate the effects of an adaptive versus non-adaptive eLearning intervention on neurophobia and neuroanatomy self-efficacy levels of first-year medical students. 


\section{CHAPTER 2}

\section{MEDICAL STUDENTS AND NEUROPHOBIA: AN ESSENTIAL DISCUSSION PRIOR TO DEVELOPING A LEARNING INTERVENTION}

2.1 Introduction. Inadequate teaching is a consistent theme in neurophobia research (Flanagan et al, 2007; Youssef, 2009; Zinchuk et al., 2010; Abulaban et al., 2015) yet a description of what is meant by inadequate teaching is lacking in the literature. Flanagan et al. (2007) conducted a survey on neuroanatomy teaching administered to medical students $(n=457)$ and found respondents rated neuroanatomy teaching as moderate to poor on a 5-point Likert scale $(1=$ very poor to $5=$ very good $)$. Students ranked 24 different instructional methods and suggested tutorials (29\%), greater exposure to neurology patients $(11 \%)$, video teaching $(2.5 \%)$, and improved online resources $(0.5 \%)$ were ways in which teaching could be improved. Another large-scale survey of medical student attitudes about neurology was conducted with 422 medical students at six different medical schools in Saudi Arabia (Abulaban et al., 2015). On a four-point Likert scale $(1=$ least difficult to $4=$ most difficult $)$, most $(85.5 \%)$ participants perceived neurology as difficult and were provided an open-ended response to why they perceive neurology as difficult. The responses were grouped into four categories: (1) $23 \%$ felt more clinical exposure was needed; (2) $16 \%$ felt teachers were inadequate or needed 
improved teaching skills; (3) 13\% wanted more neuroanatomy teaching time; and, (4) $10 \%$ wanted access to technologies such as 3D models. Similarly, a survey of medical students and residents $(n=152)$ (Zinchuk et al., 2010) reported poor neuroanatomy teaching in the first two years of medical school as contributing to neurophobia. This is concerning as most medical students (70.4\%) attribute their neurophobia to a bad teaching experience (Abulaban et al., 2015). These studies, however, did not report suggestions on how instructors could improve their lecturing skills outside of providing supplemental instructional tools. This study aims to address this gap in literature by conducting a qualitative study requesting medical students provide open responses on specific factors about neuroanatomy instruction that hinder, or may improve, their neuroanatomy learning.

Additionally, students report a lack of instructional (lecture) time as another obstacle to learning neuroanatomy (Flanagan et al., 2007; Youssef, 2009). However, lecture hours devoted to medical instruction have largely decreased in recent years due to curriculum integration (Drake et al., 2002; Drake et al. 2009). Neuroanatomy suffered the largest (18\%) decrease in lecture and laboratory hours in a survey of medical schools $(n=84)$ when converting the curriculum from systems-based to an integrated approach (Drake et al., 2002; Drake et al. 2009). This paradox of students reporting both lack of instructional time (Flanagan et al., 2007; Youssef, 2009) and inadequacy of teaching received (Flanagan et al, 2007; Youssef, 2009; Zinchuk et al., 2010; Abulaban et al., 2015) highlights the importance of investigating which specific instructional methods medical students perceive as beneficial. Medical educators need to know which instructional methods work best for first-year medical students learning neuroanatomy, 
given the documented presence of neurophobia (Schon et al., 2002; Flanagan et al., 2007; Youssef, 2009; Zinchuk et al., 2010; McCarron et al., 2014; Abulaban et al., 2015), as to make best use of the limited instructional time devoted to neuroanatomy (Drake et al., 2002; Drake et al. 2009).

Study Purpose. While inadequate teaching is a documented contributor of neurophobia in medical schools, the specific underlying reasons why students perceive neuroanatomy instruction to be inadequate are still largely unknown. One purpose of this study is to explore first-year medical students' perceptions of neuroanatomy in order to investigate the presence of neurophobia at our institution. A second purpose of this study is to explore instructional strategies that may lead to, or alternately reduce, neurophobia. Specific instructional strategies, provided by qualitative student responses in this study will be used to develop a neuroanatomy learning intervention designed to strategically mitigate neurophobia in the fourth study of this dissertation.

\subsection{Methods}

Recruitment. An email invitation from the Clinical Anatomy, Development and Examination (CADE) course director at the University of Louisville School of Medicine (ULSOM) was sent to all first-year medical students enrolled in the ULSOM CADE course $(n=155)$. This email invited students to voluntarily participate in a one-hour facilitated group discussion to provide "student attitudes towards neuroanatomy". The discussion group was entitled, "Assessing Student Attitudes Toward Neuroanatomy". Additionally, students were provided a copy of the consent preamble via email that 
explained the study purpose, voluntary participation, risks, confidentiality, and subject rights. The discussion group was scheduled at the end of the first semester of the ULSOM CADE course after the dissemination of the neuroanatomy content within the course. The discussion group was held during an available lunch session for all first-year medical students at the ULSOM. Students were informed that no names were being recorded, data would be anonymized, and their attendance at the discussion group authorized consent for this study approved by the University of Louisville Institutional Review Board.

Participants. Sixty, first year medical students $(n=60)$ voluntarily attended the session. No demographic information was recorded to provide anonymity to the students. These participants drew from the incoming ULSOM class of Fall 2017 ( $n=155)$ with an average GPA of 3.73, an average science GPA of 3.65, and MCAT scores averaging 507. The class is comprised of 54\% male and $46 \%$ female, two neuroscience majors and $6 \%$ of individuals underrepresented in medicine.

Discussion Group. One moderated, large discussion group was conducted to address the research questions: (1) Exploring student attitudes towards neuroanatomy to identify the presence of neurophobia at our institution; and (2) Investigating instructional strategies that may lead to, or alternately reduce, neurophobia. Methods of conducting the discussion group were modeled after the focus group methods described by Kruger (1994). The discussion group was led by three moderators. Two main moderators led the discussion while one research observer recorded notes during the focus group to identify body language, emotional responses and student gestures that might not have been 
otherwise recorded during the session. The research observer recorded time points when strong emotional reactions or gestures were made.

The discussion group lasted one hour. During this time, the moderators guided participants through a discussion of student attitudes towards neuroanatomy and what helps and hinders them from learning neuroanatomy. The discussion group was held in a room where each table was equipped with a microphone. Students took turns responding into the microphone for the recorded session to maintain structure during this large session.

Data Collection. A PowerPoint ${ }^{\circledR}$ presentation was used to facilitate the data collection process with the large discussion group. The participants were welcomed with a Welcome slide with the study purpose "To assess student attitudes towards neuroanatomy". A Guidelines slide informed participants the session was being recorded with a confidentiality statement, a "please be respectful and please listen respectfully as others share their views" statement, and "please know that if we interrupt you it is only to keep everyone on task and ensure everyone who wants to participate can participate" statement. A definition of Neuroanatomy and examples of "Brain and Brainstem, Spinal Cord Tracts, Basal Ganglia" were provided to participants to ensure data collected was representative of neuroanatomy, and not an alternate construct such as neuroscience. A discussion of attitudes towards neuroanatomy was guided from five predetermined main questions, and secondary probing questions. Each question allowed for 10-12 minutes of responses, and probing questions, before moving on to the next question. 
Broad questions were asked prior to probing with more specific questions, and positive questions were asked before to negative questions to reduce question bias. Moderators were instructed to maintain neutral vocal tone to minimize moderator bias, including neutral facial expressions and body posture. The moderators were also instructed to refrain from providing their own opinions during the discussion. The invitation title of the discussion reflected a neutral tone: "Assessing Student Attitudes Toward Neuroanatomy" to reduce sampling bias and discourage only students who had strong opinions, either for or against neuroanatomy, from attending. This language was intended to be welcoming of all students to be inclusive of all feedback about neuroanatomy.

To investigate student attitudes towards neuroanatomy and identify the presence of neurophobia, "How do you feel about neuroanatomy?" was asked. Probing questions, “Could you give me an example?” and, "Could you explain further?” were used for each main question. The moderators asked for alternate opinions and for input from students who did not answer as frequently. However, it was not possible to allow everyone to respond to each question before moving on due to the size of the discussion group.

To explore instructional strategies that may lead to, or alternately reduce, neurophobia, the following four main questions were used: (1) What specifically makes neuroanatomy easy?; (2) What specifically makes neuroanatomy difficult?; (3) What is working well in your neuroanatomy learning?; and, (4) What would you change to improve your neuroanatomy learning? The same probing questions "Could you give me an example?" and, "Could you explain further?" were used for each main question. 
Similarly, due to the size of the group it was not possible to allow everyone to respond to each question before continuing the discussion.

Data Analysis. Systematic review of the transcribed discussion group was modeled after grounded theory methodology (Strauss \& Corbin, 1998). Three members of the research team, representing diverse backgrounds, included one School of Medicine faculty member (Ph.D., JBC) and two graduate students (Both MS level: one Ph.D. candidate, JSB, and one pre-medical student, GBM). Researchers were instructed to set aside preconceived ideas of what may be revealed by the data and allow patterns and themes to emerge from the data.

The data analysis methods, described by Ose (2016), were conducted in Microsoft Word ${ }^{\mathrm{TM}}$ and Excel $^{\mathrm{TM}}$ for ease of utility. The recorded session was transcribed using Word ${ }^{\mathrm{TM}}$ with each question, response or comment followed by a colon (:). Student responses were numbered (i.e., Student_1:, Student_2:, etc.) and moderator questions and comments were recorded as "M." No names were recorded to protect student anonymity. A copy of the file was utilized for the initial reading of all the responses. Each researcher (JBC, JSB, and GBM) was instructed to observe, code and analyze the data independently prior to collaboration with the other researchers to minimize bias and improve credibility of the observations (Denzin, 2012). Researchers (JBC, JSB, and GBM) made note of broad themes that emerged from the data by grouping main themes of responses together. The two moderators (JBC, JSB) and research observer (GBM) independently coded the responses into data segments and grouped them into categories to observe emerging patterns and themes. 
The text from Word ${ }^{\mathrm{TM}}$ was transferred into Excel $^{\mathrm{TM}}$ using the following steps: 1) all text was copied and pasted into the top-left cell of an $\operatorname{Excel}^{\mathrm{TM}}$ spreadsheet, 2) all items in the column were highlighted and the "Data" tab was selected. Then, the "Text to Columns" option was selected, "Delimited" was chosen, "Next" was selected, and lastly "Other" was selected and a colon was inserted. The text was then separated into two columns: one column for the speaker and a second column for the moderator questions and student responses ( $\mathrm{M}$ or Student_X). Student responses were numbered in order of appearance in transcription. Time points of 5-minute intervals were recorded in the transcription.

The answers to each of the five questions (Q1-Q5) were copied and pasted into a different worksheet within the Excel ${ }^{\mathrm{TM}}$ workbook. Each worksheet was numbered Q1, Q2, Q3, Q4 or Q5. A second independent reading of all the responses was conducted with identification of quotes and supporting themes with codes created for each data segment. The research team (JBC, JSB, and GBM) reviewed the independently coded data together in a second round of data coding. Once repetitive themes were identified and no new themes surfaced from the data, the research team reached a consensus regarding the dominant themes. Finally, subthemes within each dominant domain were identified and quotes were selected as representative of each subtheme. A consensus was made by the research team of which quotes were best representative of each domain and subtheme.

Additionally, triangulation was used to validate observations in this study. Triangulation was used to validate the observations of strong emotional reactions, for example, frustration and anger in a third round of coding with the research observer (GBM) and one moderator (JSB). The research observer (GBM) noted body language, 
emotional responses and student expressions that might not have been otherwise recorded during the discussion session. The research observer (GBM) made notes of time points with these emotional responses so they could be correlated with the 5-minute intervals in the transcription. In one final meeting, the research observer (GBM) and moderators (JSB and JBC) additionally discussed these events, compared them to the transcription and their memory of the events to reach a consensus.

2.3 Results. Sixty, first-year medical students $(n=60)$ provided a total of 118 responses from the 5 main questions, along with 23 probing questions, during one-hour facilitated group discussion. Analyses of the responses identified four themes contributing to the perceived level of difficulty of neuroanatomy: Content (Table 2.1), Instruction (Table 2.2), Communication (Table 2.3), and Organization (Table 2.4). Subthemes from each of these domains emerged from the data. Tables include selected, supporting quotes as representations of student perceptions of neuroanatomy within each domain and subtheme.

Analyses of the responses also identified negative emotional responses to neuroanatomy as a result of these difficulties within the course with three subthemes emerging from the data: overwhelm, frustration, and feeling unsupported. Representative quotes from the negative emotional responses received by students are presented in Table 2.5. These negative student emotional responses led to negative feelings about neuroanatomy and may contribute to neurophobia. Each of these areas will be discussed in the following sections. 
2.3.1 Content. Four content subthemes emerged from the data as making neuroanatomy difficult: abstract nature of neuroanatomy, clinical case complexity, 3D relationships, and neuroanatomy verbiage (Table 2.1). The abstract nature of the content itself emerged as the main subtheme. Students specifically described brainstem cross-sectional anatomy, brainstem pathways and neuroanatomy tracts as not being as tangible as other anatomical structures making them inherently more difficult to learn. During the lab content, students described the anatomical differences in cortical regions of the brain between specimens as being difficult. The students interpreted this anatomical variation as "arbitrariness" or "vagueness" that needed to be accepted in order to learn neuroanatomy.

Complexity of clinical cases was another subtheme that contributed to the perceived difficulty level of neuroanatomy. Neurological clinical cases were presented in class as a Team Based Learning (TBL) exercise for the first-year students. Case presentations were interesting, yet complex, and were interpreted as overwhelming by the first-year students. Discussions that neurological lesions could have originated from more than one location contributed to neuroanatomy's "vagueness" and feelings of frustration by the students (Table 2.5).

Students also had difficulty with the $3 D$ relationships of the content. The students described the problem with seeing a cross-sectional image and not knowing where it was in 3D space. Additionally, the utilization of brain slices in different orientations (ex. coronal, sagittal, horizontal) were challenging for students to know both which orientation the slice was taken, and at which level within the brain it originated from. 
Neuroanatomical verbiage, use of terms with which the students were not yet familiar, also contributed to the perception of neuroanatomy being difficult. Students were given a comprehensive list of neuroanatomical terms for each block (or section) within the course. Novel terms combined with the description of neuroanatomy lesion locations, tract decussation points, and depiction of symptoms (ex. ipsilateral, contralateral) contributed to making neuroanatomy difficult for students.

2.3.2 Instruction. Lacking a step-by-step approach, inadequate clinical context, and structure-function correlation were the main instructional subthemes that led to the perception of neuroanatomy being difficult (Table 2.2). Students explained how the instruction was difficult to follow because it lacked a step-by-step approach. For example, blood supply and tracts were taught separately from brainstem cross-sectional anatomy. Students described these topics as puzzle pieces that did not fit together which led them to feel frustrated and overwhelmed (Table 2.5).

Brainstem cross-sectional anatomy and blood supply were also described by students as deficient in clinical context. Providing simple clinical case problems with a lesion- of one limited area of the brainstem or one specific tract- were given as examples of how to incorporate more clinical context. Dissemination of progressively more difficult clinical case problems throughout the semester, with a step-by-step walkthrough from start to finish by faculty, were also given as helpful examples to solve more complex problems like this in the future.

Another instructional challenge the students described was inadequate correlations between structure and function. They lacked a functional framework for 
these structures; thus, making learning neuroanatomy more difficult. One example the students mentioned was "kicking a soccer ball" which was used as an instructional example in teaching the motor pathways. The students wanted more of these examples of how to connect a neuroanatomy structure (tract) to a function (motor action). The crosssectional anatomy in the neuroanatomy lab was described by the students as "rote memorization" - highlighting the disjuncture between structure and function of our instruction. Students felt as if they were being thrown the information with all the pieces in the air, not knowing which to grasp, where they fit, and how to put them together; this led to students feeling frustrated, overwhelmed, and unsupported (Table 2.5).

\subsubsection{Communication. Overestimation of student knowledge, unclear student} expectations, and faculty inconsistencies were communication difficulties that made neuroanatomy unnecessarily difficult (Table 2.3). Students reported that professors overestimated student knowledge of neuroanatomy from both their prerequisite courses and their current level of understanding. Faculty frequently disseminated information above the level of students' baseline understanding which resulted in students feeling frustrated and unsupported (Table 2.5). In a team-taught neuroanatomy course, this also highlighted communication difficulties between faculty. Faculty members were unclear about what the previous faculty member covered, thereby overestimating what the students had been taught.

Another subtheme, unclear student expectations, emerged from the data as making neuroanatomy unnecessarily difficult. The students were given a large amount of material, yet it was unclear what they were specifically expected to know for their exams. 
The neuroanatomy learning objectives were vague and not as specific as their learning objectives provided in other areas of the CADE course. The students were unclear about which material was most important to focus their attention- and depth of knowledge of that material expected- leading students to feel frustrated and unsupported (Table 2.5).

Faculty inconsistencies were evident in the neuroanatomy lab contributing to making neuroanatomy difficult. Students described receiving conflicting laboratory instruction. For example, some students were taught Wernicke's area of the cerebral cortex as the entire left superior temporal gyrus from one instructor. Other students were taught the same cortical region was only the posterior aspect of the left superior temporal gyrus from another instructor. It was this ambiguity that led students to feel frustrated. Improving communication between faculty, having clear student expectations, and consistent neuroanatomy laboratory instruction will be necessary to improve students' perceptions of neuroanatomy.

2.3.4 Organization. The organizational domain describes how material organization and lecture spacing, timing, and length contributed to neuroanatomy difficulties of first-year medical students (Table 2.4). Material organization emerged as the core subtheme contributing to organizational difficulties. The order in which the neuroanatomy was presented led to students feeling overwhelmed and frustrated (Table 2.5). Neuroanatomy content was presented before the corresponding anatomy content in the course. The students lacked the base anatomy background framework for which to build the neuroanatomy content when neuroanatomy was presented prior to background anatomy lectures. Students with more experience in neuroanatomy said they felt this approach was 
“disjointed." For example, brainstem cross-sectional anatomy and tracts were placed before the location of cranial nerves exiting the external brainstem in the course. Students with prior neuroanatomy experience explained they determined which level a brainstem cross-section was located based on which cranial nerve exited the brainstem at that level. Lecture spacing, timing, and length of neuroanatomy content were also contributors to neuroanatomy difficultly. The CADE course is separated into five blocks of material throughout the semester from August to December. The bulk of the neuroanatomy content was delivered in the second and fifth blocks of the CADE course. The students described a main issue with lecture spacing was forgetting neuroanatomy material covered in the second block by the time they reached the fifth block. Having the material delivered in two short blocks instead of spreading throughout the semester, led to issues with both lecture timing and length. Neuroanatomy lectures were frequently held back-to-back with lectures often followed immediately by the lab, leaving students without sufficient time to review and digest the material between lecture and lab. A compounding factor of this organization was the length of these lectures; students were in lecture for hours at a time. This led to students feeling overwhelmed (Table 2.5).

2.3.5 Negative Emotional Responses. This domain describes the negative emotional responses students reported while learning neuroanatomy as a result of the difficulties in the four domains of content, instruction, communication, and organization. Of the total reported emotional responses $(n=16), 81 \%(n=13)$ were negative and $19 \%(n=3)$ were positive. The negative emotional responses grouped into subthemes of students feeling overwhelmed, frustrated, and unsupported while learning and studying neuroanatomy 
(Table 2.5). Students $(n=8)$ commented neuroanatomy made them feel "crushed," and "defeated," and becoming "so incredibly overwhelmed the rest of the day". This suggests this overwhelmed feeling, from learning neuroanatomy, may have influenced other areas of a student's medical school experience. A few students $(n=3)$ described feelings of frustration when trying to localize lesions. The concurrent forceful tone of voice and facial expressions described by the observer (GBM) while answering these questions supports the feelings of frustration students felt. A couple of students $(n=2)$ reported feeling unsupported by faculty through "mistakes made in lectures" and from a lack of feedback on how to correctly work through lesion problems. In summary, students learning neuroanatomy described feeling overwhelmed, frustrated, and unsupported while learning and studying neuroanatomy at our institution.

2.4 Discussion. Our open dialogue with first-year medical students demonstrated the presence of neurophobia from the negative emotional responses students described while learning neuroanatomy at our institution. This need for improvement in neuroanatomy instruction within the four domains of content, instruction, communication and organization. We found many of the same challenges with our neuroanatomy instruction as other institutions in previous studies (Youssef, 2009; Abulaban et al., 2015; Javaid et al., 2018); our students also wanted more clinical case integration, improved teaching, and explanations of 3D relationships. Similar to Javaid et al. (2018), tracts, brainstem anatomy, and clinical lesion problems were also mentioned as confusing topics for our students. However, in contrast to the descriptive study by Javaid et al. (2018), our analysis goes one step further to assess the underlying reasons for challenges in those 
areas. Our data suggested neuroanatomy content curriculum could be improved through a progression of clinical case complexity throughout the semester, more frequent distribution of concise neuroanatomy terms lists, and neuroanatomy laboratory videos to explain anatomical variability. This analysis also goes one step beyond inadequate teaching described in previous studies (Flanagan et al, 2007; Youssef, 2009; Zinchuk et al., 2010; Abulaban et al., 2015) and provides insight to what is meant by inadequate teaching. Our data suggest instruction could be improved through greater structurefunction correlation and guiding students through the thought process of solving clinical case problems. It became evident that greater communication between faculty was needed as well as improving the neuroanatomy learning objectives, making it clear what is expected of students, and adding neuroanatomy laboratory videos to improve consistency of neuroanatomy lab content. A needed organizational change to the CADE course was to have neuroanatomy content more evenly disseminated throughout the semester. These curricular changes aim to improve first-year medical student perceptions towards neuroanatomy.

The second purpose of this study was to use the specific instructional strategies, provided by qualitative student responses in this study, to develop a neuroanatomy learning intervention designed to strategically mitigate neurophobia. This study was foundational in illuminating specific aspects of neuroanatomy instruction medical students found inadequate and drove the development of the learning intervention within the scope of this dissertation. The development of this learning intervention, discussed in Chapter 5 of this dissertation, incorporated the content, instruction, communication, and 
organization domains, and associated major subthemes, described by first-year medical students from this study.

Potential limitations of this study include: lack of generalizability, moderator bias, sampling bias, and researcher bias. Data derived from a single university lacks generalization to all medical institutions due to varying implementation of first-year curriculum. ULSOM utilizes an integrated first-year curriculum with clinical examination, anatomy, neuroanatomy, and embryology taken concurrently. The focus group moderators included the CADE course director and a course teaching assistant which may have induced moderator bias. Students may not have felt entirely comfortable with sharing their negative opinions about the course in which they were enrolled. Even though the data was collected at the end of the semester, after the close of the neuroanatomy content, students may have feared negative grading repercussions because there were still a few weeks until of the end of the semester grading period. Conversely, students may have shared overly positive opinions to impress the course director. Convenience sampling may have impacted the study results, as it is not known if students with strong opinions, either for or against neuroanatomy, were the only ones to participate. Further, the large group setting may have disincentivized students who dislike speaking in public from sharing. Researcher bias was minimized with instructions to maintain neutral facial expressions, body posture, and tone. During coding, researchers were reminded to allow patterns and themes to emerge from the data instead of trying to fit the data into preconceived patterns and themes. Additionally, due to time constraints the researchers were able to schedule only one discussion group session. The large size of the discussion group limited the number of student responses and prevented grounded 
theory methodology to be used fully. Grounded theory methodology is best used with smaller, successive focus groups that are questioned until there is redundancy in participant responses.

In summary, this in-depth qualitative analysis of first-year medical students' perceptions toward neuroanatomy provides enlightenment on reasons why neuroanatomy is often perceived to be difficult and suggests specific curricular changes for improving neuroanatomy instruction. This study provides evidence for neurophobia at this institution, supports further investigation of neurophobia in this dissertation and informs the development of educational interventions designed to mitigate it. Student reflections provide insight into how we can teach and organize neuroanatomy differently to minimize neurophobia and increase interest and comprehension. Development of a novel survey to explore and measure the construct of neurophobia will be described in the next study, Chapter 3 of this dissertation. This survey will be used to assess the efficacy of the learning intervention, the development of which was informed from this study, designed to reduce neurophobia in Chapter 5 of this dissertation. 


\section{TABLE 2.1}

\begin{tabular}{|c|c|}
\hline \multicolumn{2}{|r|}{ Content Domain } \\
\hline Subtheme & Supporting Key Quotes \\
\hline $\begin{array}{l}\text { Abstract } \\
\text { Nature }(n=7)\end{array}$ & $\begin{array}{l}\text { Student_22: ...it doesn't click right away and if we had a picture with just } \\
\text { a... brain and that red pane instead of just being like ... anterior } \\
\text { commissure... and I'm just like ok where the hell is that...where am I? } \\
\text { Student_34: I think just one thing that makes neuro difficult is just there are } \\
\text { a lot of...arbitrariness when it comes to areas of the brain...the brain is just } \\
\text { very vague I guess...it's one of those things that you have to kind of swallow } \\
\text { that you know there's a lot of variation in areas and structure }\end{array}$ \\
\hline $\begin{array}{l}\text { Clinical Case } \\
\text { Complexity } \\
(n=7)\end{array}$ & $\begin{array}{l}\text { Student_10: ...the clinical cases after quizzes and we would try to localize } \\
\text { lesions but then he was like, every answer is correct...like it could be a lesion } \\
\text { anywhere } \\
\text { Student 40: ... the clinical exercises that we do were...really intricate } \\
\text { cases. While those are really interesting, it is important to know there are } \\
\text { always going to be really complicated cases }\end{array}$ \\
\hline $\begin{array}{l}\text { 3D } \\
\text { Relationships } \\
(n=4)\end{array}$ & $\begin{array}{l}\text { Student_16: ...I think that (having } 3 D \text { models) would be a lot easier than } \\
\text { having to translate } 2 D \text { images from a pdf into where they are spatially } \\
\text { Student_1: I think through things three-dimensionally and I think that a lot } \\
\text { of the slides, where we're at, kind of lacks that...it's a little harder to } \\
\text { understand when you don't know where things are three-dimensionally }\end{array}$ \\
\hline $\begin{array}{l}\text { Neuroanatomy } \\
\text { Lexicon }(n=5)\end{array}$ & $\begin{array}{l}\text { Student_24: We didn't really learn very much in the first few neuro brain } \\
\text { identification labs... you are getting engrossed in the terminology and you're } \\
\text { like, I don't know if it's this ridge or that ridge... } \\
\text { Student_44: when I'm reading slides the use of contralateral, ipsilateral, } \\
\text { bilateral...things can get a little bit convoluted when he's up there talking } \\
\text { and using all three of those words in one sentence and you still aren't quite } \\
\text { sure where the lesion is even coming from } \\
\text { Student_42:... when you are like just drowning in terms } \\
\text { Student_43: ... when you are trying to understand like the basics of } \\
\text { something and then all these words are coming, like corticobulbar, and all of } \\
\text { those things like, wait, what is that again? }\end{array}$ \\
\hline
\end{tabular}


TABLE 2.1 Content Domain. Samples of qualitative student responses representative of the content domain. Qualitative data analysis supports four subthemes of abstract nature, clinical case complexity, 3D relationships, and neuroanatomy lexicon contributing to perceived student difficulties with the neuroanatomy content. (Student comments were anonymized and numbered in the order of appearance within the transcript) 


\section{TABLE 2.2}

\begin{tabular}{|c|c|}
\hline \multicolumn{2}{|r|}{ Instruction Domain } \\
\hline Subtheme & Supporting Key Quotes \\
\hline $\begin{array}{l}\text { Lacking } \\
\text { Step-by- } \\
\text { Step } \\
\text { Approach } \\
(n=14)\end{array}$ & $\begin{array}{l}\text { Student_11: ...when we actually had the MRI slices as well as the true brain } \\
\text { slices that he went over and actually said like, "This is the Putamen" and that } \\
\text { was incredibly helpful } \\
\text { Student_42: I think there are some teaching strategies that could be... adopted } \\
\text { by another lecturer....in general, I think like dumbing down (simplifying) the } \\
\text { material a little but-even though we are all really smart it just really helps } \\
\text { when she's like ascending-and by that I mean going from here to here... } \\
\text { Student_59: I think like putting all the different pieces of neuroanatomy } \\
\text { together so like the blood supply versus like where it's physically located on the } \\
\text { slide versus, you know, just like all of those little pieces to summarize them and } \\
\text { kind of like make them fit together a little better }\end{array}$ \\
\hline $\begin{array}{l}\text { Absent } \\
\text { Clinical } \\
\text { Context } \\
(n=6)\end{array}$ & $\begin{array}{l}\text { Student_8: I kind of wish we had more real-life examples... } \\
\text { Student_41: I think one of the big things, like overarching themes that makes } \\
\text { neuroanatomy difficult is just lacking clinical context...one example would be } \\
\text { when we learn about several different pathways and we saw them on the slides } \\
\text { but we didn't really like learn a lot about all the different pieces and now we } \\
\text { are kind of starting to put those pieces in there...it was just really hard because } \\
\text { we were just like rote memorizing words out of a slide...integrated is helpful }\end{array}$ \\
\hline $\begin{array}{l}\text { Missing } \\
\text { Correlation } \\
\text { Between } \\
\text { Structure } \\
\text { and } \\
\text { Function } \\
(n=5)\end{array}$ & $\begin{array}{l}\text { Student_37: One thing that I think really helps me to...learn the things on the } \\
\text { brainstem slices is when we're in lecture...throw up a picture of where it is at } \\
\text { in the brainstem, here it is, and this is what it does...this is what its purpose is, I } \\
\text { think that helps me more so than just separating the two issues } \\
\text { Student_4: ...the tracts we learned, weren't really explained what they } \\
\text { did...they were just like, this is a tract, right...for me that was sort of difficult to } \\
\text { put in my memory because it was some random word and some random blob on } \\
\text { some random slide ... so I didn't have like a context to put any of this in and } \\
\text { learn it }\end{array}$ \\
\hline
\end{tabular}


TABLE 2.2 Instruction Domain. Samples of qualitative student responses representative of the instruction domain. Data analysis supports three subthemes of lacking a step-by-step approach, absent clinical content, and missing correlation between structure and function leading to student perception of instructional challenges with learning neuroanatomy (Student comments were anonymized and numbered in the order of appearance within the transcript) 


\section{TABLE 2.3}

\begin{tabular}{|l|l|}
\hline \multicolumn{2}{|c|}{ Communication Domain } \\
\hline Subtheme & Supporting Key Quote(s) \\
\hline $\begin{array}{l}\text { Overestimation } \\
\text { of Student } \\
\text { Knowledge } \\
(n=2)\end{array}$ & $\begin{array}{l}\text { Student_39: ... since we have so many different lecturers for neuroanatomy } \\
\text { material and it seems like they don't communicate enough with each } \\
\text { other...because one lecturer will always assume that we know something } \\
\text { that we don't know and then like they start off... and then five minutes in and } \\
\text { you're already lost and the whole fifty-minute lecture is completely useless } \\
\text { because they think that you know something that you don't know... }\end{array}$ \\
\hline $\begin{array}{l}\text { Unclear } \\
(n=2)\end{array}$ & $\begin{array}{l}\text { Student_52: What is the jist of what you were supposed to get from the last } \\
\text { four slides...? }\end{array}$ \\
$\begin{array}{l}\text { Student_36: ...in the lectures there will be like here 's like three slides of } \\
\text { this but like, oh, you don't need to know that, so it's just like I have trouble } \\
\text { when I am studying and I get to a point and it's like oh like they're } \\
\text { discussing that more later so I like I don't really know where to go with that } \\
\text { when it's like we're coming to it later or you don't need to worry about it }\end{array}$ \\
$\begin{array}{l}\text { Student_45: So, I like live, eat and breathe the learning objectives for } \\
\text { anatomy because they are probably saving my life, but the neuroanatomy } \\
\text { learning objectives...seem to be like very broad and sometimes they don't } \\
\text { even seem to be like over what we're doing... }\end{array}$ \\
$\begin{array}{l}\text { Student_13: And then occasionally we'd get different input from different } \\
\text { instructors so that was hard to overcome as well }\end{array}$ \\
$\begin{array}{l}\text { Student_14: Ifeel like we get more guidance that is more consistent in } \\
\text { gross anatomy...so there is a lot of information available for neuroanatomy- } \\
\text { but it's not a consensus (from the faculty) all the time... }\end{array}$ \\
$\begin{array}{l}\text { Inconsistencies } \\
(n=2)\end{array}$
\end{tabular}


TABLE 2.3 Communication Domain. Samples of qualitative student responses representative of the communication domain. Data analysis supports three subthemes of overestimation of student knowledge, unclear expectations, and faculty inconsistencies leading students to perceive neuroanatomy as unnecessarily difficult (Student comments were anonymized and numbered in the order of appearance within the transcript) 
TABLE 2.4

\begin{tabular}{|c|c|}
\hline \multicolumn{2}{|r|}{ Organization Domain } \\
\hline Subtheme & Supporting Key Quote(s) \\
\hline $\begin{array}{l}\text { Material } \\
\text { Organization } \\
(n=14)\end{array}$ & $\begin{array}{l}\text { Student_38: I found it difficult several times we were given } \\
\text { neuroanatomy lectures prior to having the gross anatomy lectures and I } \\
\text { thought that was just useless because I couldn't imagine the neuro if I } \\
\text { didn't know the anatomy yet } \\
\text { Student_42: ...some of the (PowerPoint) slides would have five bullet } \\
\text { points and what they intended for was the last word to fall under the } \\
\text { first one-but they were all in the same indentation...then also } \\
\text { sometimes the bullet points were meant to be a pathway going from one } \\
\text { to the next... but I didn't pick up on that because it wasn't mentioned in } \\
\text { the slide that it was supposed to be a pathway moving from one bullet } \\
\text { point to the next } \\
\text { Student_46: ... it seemed really odd that we are only now getting an } \\
\text { introduction to the cranial nerves because I use them to orient myself at } \\
\text { like all the different (brainstem) levels...like where they come off the } \\
\text { brainstem while we are doing external brainstem, um, like that didn't } \\
\text { make sense to me...it seemed a little bit disjointed in the way that I tend } \\
\text { to approach it... }\end{array}$ \\
\hline $\begin{array}{l}\text { Lecture } \\
\text { Spacing } \\
(n=9)\end{array}$ & $\begin{array}{l}\text { Student_17: The biggest thing I feel like would be helpful is if the } \\
\text { blocks weren't as spread out, because I feel like when we started block } \\
\text { five, I had to take a couple days to like review block two... so it would } \\
\text { be a little more helpful if they were a little closer together in time } \\
\text { Student_54: I think that block five is extremely front loaded... those } \\
\text { first two weeks we probably had more lectures than some blocks even } \\
\text { had as a whole...I found myself trying to just like not drown in the } \\
\text { information... }\end{array}$ \\
\hline $\begin{array}{l}\text { Lecture } \\
\text { Timing } \\
(n=4)\end{array}$ & $\begin{array}{l}\text { Student_20: I thought that the first neuro block labs were kind of rough } \\
\text { because we would have a lecture but then we would go into the lab } \\
\text { right after } \\
\text { Student_48:...you go into one PowerPoint, assuming that you know the } \\
\text { previous one, but there's been fifteen minutes in between instead of } \\
\text { having a day (between lectures) }\end{array}$ \\
\hline $\begin{array}{l}\text { Lecture } \\
\text { Length } \\
(n=3)\end{array}$ & $\begin{array}{l}\text { Student_52: I think having four straight hours of neuroanatomy lecture } \\
\text { in the morning is exhausting and disheartening and nearly impossible } \\
\text { Student_48: ...the large days of neuro were really killer }\end{array}$ \\
\hline
\end{tabular}


TABLE 2.4 Organization Domain. Samples of qualitative student responses representative of the organization domain. Data analysis supports four subthemes of material organization, lecture spacing, timing, and length contributing to organizational difficulties leading neuroanatomy to be perceived as difficult (Student comments were anonymized and numbered in the order of appearance within the transcript) 


\section{TABLE 2.5}

\begin{tabular}{|c|c|}
\hline \multicolumn{2}{|r|}{ Student Negative Emotional Responses } \\
\hline Subtheme & Supporting Key Quote(s) \\
\hline $\begin{array}{l}\text { Overwhelmed } \\
(n=8)\end{array}$ & $\begin{array}{l}\text { Student_40: really intricate cases were...extremely overwhelming to } \\
\text { hear that the lesion could have been in like thirty different places } \\
\text { most of which we never heard of before... } \\
\text { Student_2: Well, I came in kind of really excited about } \\
\text { neuroanatomy because I like the brain, and that is sort of what I've } \\
\text { always liked, but I was quickly crushed, or defeated, or like quickly } \\
\text { starting hating this } \\
\text { Student_5: I was really intimidated and overwhelmed and I thought, } \\
\text { oh, this is horrible } \\
\text { Student_20: I've never even like worked with brains before in my } \\
\text { life so it's just really overwhelming } \\
\text { Student_51: I became so incredibly overwhelmed that the rest of the } \\
\text { day-I think we had two other lectures that day-and the next day } \\
\text { there were three-Ijust lost it }\end{array}$ \\
\hline $\begin{array}{l}\text { Frustrated } \\
(n=3)\end{array}$ & $\begin{array}{l}\text { Student_10: Ijust had a lot of frustration with that specific time } \\
\text { where we'd try to localize lesions and then we never got a specific } \\
\text { answer on how that would present } \\
\text { Student_42: So where is this lesion? It's like I can't remember forty } \\
\text { minutes ago I don't even remember my name right now... }\end{array}$ \\
\hline $\begin{array}{l}\text { Unsupported } \\
(n=2)\end{array}$ & $\begin{array}{l}\text { Student_60: It would be helpful to be able to have that (practice } \\
\text { questions) like feedback on if you do get it wrong here's why so you } \\
\text { can work through it and use it to help increase your learning rather } \\
\text { than just telling you how much you don't know } \\
\text { Student_35:...there was like not a day we went home for the last } \\
\text { few weeks where we didn't get something where it was like this } \\
\text { mistake was made in lecture today and by the time it was done-I } \\
\text { know a lot of people probably looked through the notes and kind of } \\
\text { like solidified that information and it's a lot harder to relearn } \\
\text { information a different way after you've been given the information } \\
\text { incorrectly }\end{array}$ \\
\hline
\end{tabular}


TABLE 2.5 Student Negative Emotional Responses. Samples of qualitative student responses demonstrating the overall results of the study. The four domains of content, instruction, communication, and organization led to negative emotional responses of students towards neuroanatomy. Students were left feeling overwhelmed, frustrated, and unsupported while learning neuroanatomy in their first-year medical student course. (Student comments were anonymized and numbered in the order of appearance within the transcript) 


\section{CHAPTER 3}

\section{PREDICTORS OF NEUROANXIETY AMONG MEDICAL STUDENTS IN AN INTEGRATED MEDICAL CURRICULUM}

3.1 Introduction. Medical neuroanatomy is one of the most feared subjects by medical students. This fear of neuroanatomy in medical students led to the development of the term neurophobia, the debilitating "fear of the neural sciences and clinical neurology...due to the student's inability to apply their knowledge of basic sciences to clinical situations" (Jozefowicz, 1994, p. 328) in 1994. Critics of neurophobia have considered the term itself as not being "not totally serious" (Schon et al., 2002; p. 557) and to-date no known in-depth conceptualization of this construct has been conducted. Additionally, a literature search to-date found no psychometrically validated scales measuring neurophobia. Neurophobia research has focused on finding the factors associated with it (Flanagan et al, 2007; Youssef, 2009; Zinchuk et al., 2010; Abulaban et al., 2015) and investigating educational interventions and strategies to mitigate it (Hudson et al., 2006; Anwar et al., 2015; Shiels et al., 2017; Javaid et al., 2018; Sotgiu et al., 2020), yet the construct itself has yet to be explored in-depth. The first study in this chapter aims to fill the gap in the literature by conceptualizing neurophobia prior to developing a novel instrument to measure it. Conceptualization of neurophobia as a type 
of anxiety, called neuroanxiety, was based on the seminal psychological research on anxiety (Lang, 1971; Hodgson \& Rachman, 1974; Lang, 1978; Ost \&Hugdahl, 1981; Norton \& Johnson, 1983; Michelson, 1986). The first study in this chapter will contribute to the literature by providing a theoretical framework for neuroanxiety, develop a novel instrument to measure it, and provide data for initial validity and reliability evidence for the instrument. This instrument will provide medical educators with a tool to measure neuroanxiety differences in subpopulations of medical students in this study; additionally, this scale will be used to measure the efficacy of educational strategies designed to reduce it in Chapter 5 of this dissertation.

A major concern with neurophobia in medical education is the gender disparity in neurological medicine with fewer female medical students pursuing careers in neurology. In a 2018 study surveying 1,712 academic neurologists, 30.8\% (n=528) were women and 69.2\% ( $n=1184)$ were men (McDermott et al., 2018). While a gender disparity exists in many medical school faculty appointments, a particularly large gender gap exists in neurological medicine (38.4\%) when compared to all other medical specialties $(16.7 \%)$ (Jena et al., 2015; McDermott et al., 2018). Concurrently, a report by the American Academy of Neurologists (Freeman et al., 2013) shows a neurologist shortage with a projected need for $19 \%$ more neurologists by 2025 . The American Academy of Neurology created a Gender Disparity Task Force (GDTF) to address the problem (AAN, 2017). Increasing the number of female neurological specialists could help supplement the current shortage of neurologists and neurosurgeons in the United States while concurrently narrowing the gender gap. It is unknown whether neurophobia may be differentially affecting female medical students, thereby preventing them from pursuing a 
neurology career. Studies report conflicting results regarding neurophobia and gender (Kam et al., 2013; Shiels et al., 2017). Kam et al. (2013) reported gender is one of the main factors that predicts neurophobia in a study of medical students $(n=158)$ and residents $(n=131)$. A later study by Shiels et al. (2017) reported no significant gender difference in neurophobia in a study of medical students $(n=446)$. One purpose of this study is to add to the depth of literature on investigating gender differences in neurophobia (neuroanxiety) among medical students to explore if neuroanxiety is preferentially affecting female medical students.

Additionally, these studies by Kam et al. (2013) and Shiels et al. (2017) report differing neurophobia scores depending on a student's medical school class and neuroanatomy knowledge level. Kam et al. (2013) reported novice medical students, with low neuroanatomy knowledge, scored higher on neurophobia surveys. Along with female gender, the Kam et al. (2013) study described how low neuroanatomy interest and low neuroanatomy knowledge predicted neurophobia in a multivariate analysis. Kam et al. (2013) included questions about neuroanatomy knowledge level in their survey. Medical students $(n=158)$, with low neuroanatomy knowledge, reported a higher prevalence (47.5\%) of neurophobia than residents ( $n=131)$, with high neuroanatomy knowledge, showing a lower prevalence (36.6\%) of neurophobia. Alternatively, Shiels et al. (2017) observed a higher prevalence of self-reported neurophobia (26\%) in second-year medical students when compared to first year-medical students (19\%). Although Shiels et al. (2017) did not ask respondents to rate their neuroanatomy knowledge, presumably second-year medical students would have more neuroanatomy knowledge than first-year medical students. These conflicting results suggest further investigation is needed into 
medical school class, with differing neuroanatomy knowledge levels, and neurophobia. This study adds to the breadth of literature by exploring the differences in neurophobia (neuroanxiety) among first-year and upper-class medical students.

Other factors contributing to neurophobia have been explored in the research with neuroanatomy difficulty level and complex physical exam (Youssef, 2009) with poor teaching experiences identified as major contributors (Schon et al., 2002; Abulaban et al., 2015). Inadequate teaching is a consistent theme with neurophobia research (Schon et al., 2002; Flanagan et al, 2007; Youssef, 2009; Zinchuk et al., 2010; Abulaban et al., 2015; Javaid et al., 2018). Yet, details about these teaching experiences and, alternatively, instructional strategies that could improve neuroanatomy instruction are lacking in the literature. The second study in this chapter aims to contribute depth to the existing literature by exploring specific instructional predictors of neurophobia (neuroanxiety). The purpose of this second study is to investigate variables that predict neuroanxiety in a medical student population. This study will explore other variables, such as gender, premedical experiences, and neuroanatomy's reputation, in addition to specific instructional predictors of neuroanxiety. Premedical neuroscience and/or neuroanatomy experience, and specific instructional variables, have not yet been investigated as a predictor of neuroanxiety and this study aims to address this gap in the literature. Additionally, exploring specific instructional predictors of neuroanxiety will inform the curriculum development and learning strategies designed minimize the effects of neuroanxiety.

The first study, Study 1 Neuroanxiety Scale Development and Initial Validation, will explore the development of the survey and steps taken to evaluate scale validity. The 
exploration of the neurophobia construct, its conceptualization as neuroanxiety, and a novel neuroanxiety scale will be developed and used to explore the differences in neuroanxiety between medical school classes and binary gender. The second study, Study 2 Predictors of Neuroanxiety will be described next. Specific instructional predictors, along with other predictors found in the literature, will be described and investigated in this second study. Subsequently, an overall discussion for both studies will complete the chapter.

\subsection{STUDY 1 NEUROANXIETY SCALE DEVELOPMENT AND INTIAL}

\section{VALIDATION}

3.2.1 Study Purpose. The purpose of this study is to develop and explore the initial validation of an instrument designed to measure neuroanxiety in medical students. First, the neurophobia construct will be explored as to whether it could more accurately be described as a subtype of anxiety, called neuroanxiety. Conceptualization of neuroanxiety was based the seminal psychological research on anxiety during the development of this novel instrument (Lang, 1971; Hodgson \& Rachman, 1974; Lang, 1978; Ost \& Hugdahl, 1981; Norton \& Johnson, 1983; Michelson, 1986). This present study will fill this gap in the literature by providing a theoretical framework for neuroanxiety, develop a novel instrument to measure it, and provide data for initial validity and reliability evidence for the instrument. A second purpose of this study is to use the novel neuroanxiety scale to add to the breadth of literature by exploring the differences in neuroanxiety between medical school classes and binary gender at our institution. Lastly, the neuroanxiety scale 
developed in this study will be used to assess the efficacy of a learning intervention designed to reduce neurophobia in Chapter 5 of this dissertation.

\subsubsection{Background}

Neuroanxiety defined. An investigation into the original neurophobia construct was conducted. Neurophobia's definition is: "The debilitating fear of the neural sciences and clinical neurology...due to the student's inability to apply their knowledge of basic sciences to clinical situations" originated in 1994 (Jozefowicz, 1994, p. 328). Considering critics have described this term as being "not totally serious" (Schon et al., 2002; p. 557), an alternate definition was explored. Kam et al. (2013) defined neurophobia as a combination of low neurology confidence and high perceived difficulty level as scored on a 5-point Likert scale (the instrument itself will be reviewed in the Measurement Issues section below). Kam et al. (2013) interpreted "lack of confidence as intimidation and anxiety which is a prominent symptom of neurophobia" (p. 560). No other competing definitions of neurophobia were found in the literature.

Many studies of neurophobia (Flanagan et al., 2007; Risdale et al., 2007;

Youssef, 2009; Zinchuk et al., 2010; McCarron et al., 2014; Pakpoor et al., 2014; Javaid et al., 2018) cite the original definition by Jozefowicz as the definition of neurophobia. Kam et al. (2013) described interpreting the "lack of confidence" in neurology as an anxiety. This study will consider and explore neurophobia and describe how neurophobia may be more accurately defined as a type of anxiety. In this study, neuroanxiety will be defined as: An anxiety towards learning neuroanatomy and applying neuroanatomical 
concepts to a clinical setting. The conceptualization of neurophobia as neuroanxiety is described below.

Neurophobia: Phobia or Anxiety? Initially, the diagnostic criteria for phobias and anxiety disorders in the DSM-5 (5th ed.; DSM-5; American Psychiatric Association, 2013) were reviewed to determine which term more accurately describes the debilitating fear aspect of neurophobia, namely: anxiety or phobia. A case study by Giles (2010) found neurophobia elicited clinical levels of anxiety according to the State Trait Anxiety Inventory (Spielberger et al., 1989) in one medical student. However, no other published psychological measures of anxiety or phobia were used to validate whether neurophobia was more appropriately categorized as a phobia or an anxiety. This exploration, detailed below, explores the diagnostic criteria of phobias and anxiety and proposes this construct more accurately reflects a type of anxiety.

Phobias must contain the following diagnostic criteria: unreasonable fear, immediate anxiety response out of proportion with the actual danger, avoidance or endures with extreme distress, life-limiting, six months in duration and not caused by another disorder or medication (APA, 2013). The categorization of this construct as a phobia may be extreme as most students are not life-limited, for example, unable come to class and drop out of medical school when suffering from neurophobia. The literature was explored to investigate if medical students avoid class, and subsequently drop out of medical school, because of neurophobia. To-date no literature was found providing evidence of increased medical student drop-out rates due to neurophobia. Ahamdy et al. (2019) evaluated 89 studies detailing medical student attrition and found most medical 
students fail or dropout due to personal reasons unrelated to either anxiety or neurophobia. If neurophobia is a true phobia, it could be theorized that more medical students would be dropping out of medical school at higher rates due to the increasing prevalence of neurophobia (Anwar et al., 2015; Shiels et al., 2017; Javaid et al., 2018; Sotgiu et al., 2020).

In a case study of one medical student, Giles (2010) found neurophobia elicited clinical levels of anxiety according to the State Trait Anxiety Inventory (Spielberger et al., 1989), a validated mental health diagnostic tool for anxiety. Appreciating this is a case study of one medical student, yet, if neurophobia elicits anxiety on a published and validated anxiety disorder scale, one could hypothesize neurophobia may be more accurately classified as an anxiety and not a phobia. The diagnostic criteria for an (generalized) anxiety disorder is having three of the following symptoms (for six months or more) according to the DSM-5 (5th ed.; DSM-5; American Psychiatric Association, 2013): Restlessness or feeling on edge, easily fatigued, difficulty concentrating, irritability, muscle tension, or sleep disturbance impairing occupational functioning not caused by another disorder or medication. Considering medical students may have difficulty concentrating or feeling on edge when experiencing neurophobia, which are diagnostic anxiety symptoms, exploration into whether neurophobia could be more accurately described as a type of anxiety will be explored.

Conceptualizing neurophobia as neuroanxiety. A review of anxiety models by psychological researchers guided the conceptualization of neurophobia as neuroanxiety (Lang, 1971; Lang, 1978; Barlow, 1985; Hodgson \& Rachman, 1974; Ost \&Hugdahl, 
1981; Norton \& Johnson, 1983; Michelson, 1986; Spitzer et al., 2006). Lang described a three-dimensional model of anxiety as having cognitive, behavioral, and physiological factors (Lang, 1971; Lang, 1978). Cognitive, behavioral, and physiological factors of anxiety are rooted in an evolutionary hardwired response that processes emotionally significant stimuli to initiate a behavioral response. The hypothalamus-pituitary-adrenal (HPA) axis, hippocampus, amygdala and the medial prefrontal cortex (mPFC) are involved in the circuitry responsible for these symptoms and behaviors (Martin et al., 2009; Shin \& Liberzon, 2010; Ferrari et al., 2011). The following cognitive, behavioral, and physiological factors of anxiety will be reviewed and compared with medical students experiencing neurophobia in the literature.

Cognitive. Cognitive aspects of anxiety include symptoms such as worry, rumination of fearful or negative thoughts, or overestimation of negative future events. Medical school is a stressful environment, and one study shows most $(81.1 \%)$ medical students $(n=94)$ at one institution exhibited high levels of general anxiety (Kim, 2016). Cognitive anxiety may affect encoding of novel memories within the hippocampal circuitry (Ferrari et al., 2011) which may subsequently influence memory and a student's performance on examinations. Studies show medical students are affected by test-anxiety on high-stakes examinations (Kim, 2016) and this high level of anxiety may actually demotivate medical students (Saravann \& Wilks, 2014). While no studies to-date were found exploring neurophobia and medical student neuroanatomy test-anxiety directly, studies report a correlation between lack of interest (amotivation) and neurophobia (Kam et al., 2013; Shiels et al., 2017; Javaid et al., 2018). It could be hypothesized that neurophobia, 
considered as a type of anxiety, may influence this lack of interest (amotivation) in neuroanatomy. Kam et al. (2013) found lack of neuroanatomy interest was one predictor of neurophobia.

Behavioral. Behavioral features of anxiety include avoidance, restlessness, agitation or the desire escape from anxiety producing situations are also hardwired from this evolutionary circuitry (Tachere \& Modirrousta, 2017; Kirk et al., 2019). Rachman and Hodgson (1974) initially described the interrelationship between fear and avoidance. Rachman later clarifies, "in fears acquired by a conditioning process...the components that will be most prominent are the psychophysiological and behavioral" (1978. p. 198). Behavioral aspects of anxiety, such as avoidance, are stronger if one participates in an active (conditioning) process. One may argue that attending medical school neuroanatomy lecture is a conditioning process, of which medical students attend on a regular basis and consistently rate as inadequate (Flanagan et al, 2007; Youssef, 2009; Zinchuk et al., 2010; Abulaban et al., 2015). Possibly medical students exhibiting neurophobia may avoid studying, or procrastinate, neuroanatomy. Academic procrastination among medical students $(n=400)$ in one study is rated as high $(47.9 \%)$ and students reported a negative relationship with their academic achievement (Hyat et al., 2020). Potentially related to avoidance, Kam et al. (2013) found lack of general knowledge, as well as interest, as predictors of neurophobia. One might theorize that medical students exhibiting a lack of interest, and subsequent lack of knowledge from avoiding pursuing neuroanatomy education, could be concurrently exhibiting neurophobia. 
Physiological. Anxiety produces physiological symptoms including sweating, increased heart rate, increased breathing rate or feeling jittery. Studies from the literature show more than half $(53.9 \%)$ of medical students $(n=154)$ at one institution (Saravann \& Wilks, 2014) and most (81.1\%) medical students ( $n=94)$ at another institution exhibited high levels of general anxiety (Kim, 2016). Saravann and Wilks (2014) also reported medical students experiencing physiological and psychological distress from their anxiety. Every medical student is required to take a neuroanatomy course. It is unknown if neurophobia is a distinct form of anxiety or an expression of anxious medical students learning neuroanatomy because of the concurrent high levels of general anxiety (Saravann \& Wilks, 2014; Kim, 2016) and neurophobia reported at medical schools (Kam et al., 2013; Shiels et al., 2017; Javaid et al., 2018).

In summary, it is hypothesized that neurophobia could be more accurately described as a type of anxiety because of the cognitive, behavioral and physiological behaviors reported by medical students (Saravann \& Wilks, 2014; Kim, 2016; Shiels et al., 2017). Additionally, qualitative data from our discussion group in Chapter 2 of this dissertation yielded student responses consistent with symptoms of anxiety. Student responses supporting each factor of the neuroanxiety construct were counted and examples of key supporting quotes were provided (Table 3.2). Physiological effects described by students were feeling jittery, exhausted and angry when explaining their experiences in neuroanatomy. Negative thoughts and memory difficulties were the cognitive symptoms described. There was a tone of anger and frustration in the student comment, I don't even remember my name right now. Students even described 
demonstrating avoidance behavior when it came to studying for or attending neuroanatomy lectures. Our qualitative data analysis, as well as the literature on anxiety, suggests neurophobia may more accurately described as a type of anxiety, called neuroanxiety.

Distinguishing neuroanxiety from neuroscience anxiety. The fields of neuroanatomy and neuroscience are areas whose content appears to be analogous to a novice learner such as a first-year medical student. When considering defining neuroanatomy for the measurement of neuroanxiety, however, these terms must be differentiated to avoid confusion. Neuroanatomy is considered the structural elements, neurons and their structural connectivity with associated glial cells, comprising the nervous system (DuqueParra, 2002; Fischl et al., 2002; Rushmore et al., 2020). Neuroscience is a broad term that reflects the interdisciplinary nature of brain and spinal cord research and including, but not limited to, neuroanatomy, neurophysiology, molecular biology, and other disciplines (Duque-Parra, 2002). We defined neuroanatomy as anatomical connectivity within the brain and spinal cord. Neuroanatomy examples provided to respondents were the locations of cell bodies with associated brainstem nuclei, synapses, and decussation points of specific tracts (corticospinal tract, spinocerebellar tract, spinothalamic tract, etc...). The scale specifically describes lesion location isolation within the brain and spinal cord- utilizing clinical case scenarios- as neuroanatomy. We defined neuroscience as the molecular study of neurons. The study of synapses, axonal physiology, neurotransmitters with receptor physiology, and microscopic neuronal connectivity were provided as examples of neuroscience (Duque-Parra, 2002; Fischl et al., 2002; Rushmore 
et al., 2020). Care was taken to provide students a clear definition of neuroanatomy to help define the variable and preserve content validity of the scale. The researcher's intention is to measure anxiety towards neuroanatomy and not capture student anxiety towards neuroscience.

Measurement Issues. Much of the research measuring neurophobia (Schon et al., 2002; Flanagan et al., 2007; Risdale et al., 2007; Youssef, 2009; Zinchuk et al., 2010; McCarron et al., 2014; Pakpoor et al., 2014; Javaid et al., 2018) is derived from a single scale (Schon et al., 2002). The scale developed by Schon et al. (2002) uses a 5-point Likert scale to measure levels of: current interest, level of knowledge, perceived difficulty and patient care confidence in seven different medical specialties. This instrument may not be accurately capturing fear of neuroscience and one's ability to learn and apply neuroanatomy in a clinical setting as based on the original definition (Jozefowicz, 1994; p. 328). Fear of a subject may affect the level of one's interest, knowledge, perception of difficulty and confidence. However, one might argue that low interest and low knowledge level in a subject may not directly translate to feelings of fear and anxiety. For example, one initially may have low interest and knowledge in a subject, but with focused time and effort could expand their knowledge, subsequently naturally increasing their interest. Patient care confidence could reflect a medical student's lack of experience, deficiencies in their physical examination skills, or for other reasons outside of feelings of fear or anxiety. Consequently, this scale may not be as accurately capturing fear of neuroanatomy as the scale intended. One may be measuring level of interest, knowledge, and physical examination skills of medical students among different medical 
specialties. Neurophobia scales developed in the literature, based on the Schon et al. (2002) scale, were reviewed and improvements authors made to the original neurophobia scale are described below.

Flanagan et al. (2007) improved the Schon et al. (2002) scale with additional questions to explore reasons why neurology is difficult for medical students. The additional question, Why is neurology difficult, was scored on a 5-point Likert scale (1= not at all to 5=major contributor). Students were asked to rate the following reasons why neurology is so difficult: neuroanatomy content, neuroscience, diagnostic complexity of neurological conditions, limited patient exposure in medical school, insufficient teaching, poor teaching, and neurological examination. The meanings of the terms insufficient and poor teaching were not described, and their definitions may have been unclear to the respondent. Is unknown if insufficient teaching means lack of time for lecture or is more reflective of the instructor's teaching ability (which may also be captured in the poor teaching item). Flanagan et al. (2007) broadened the scale, from comparing different medical specialties, to investigating potential reasons why neurology is perceived as difficult. One limitation of this scale was the lack of open-ended questions for students to provide their own reasons they may perceive neurology as difficult.

Zinchuk et al. (2010) filled this literature gap by further developing their scale, based on both the Schon et al. (2002) and Flanagan et al. (2007) scales, with an additional open-ended question where participants could provide their own reason(s) neurology is difficult. This allowed researchers to capture qualitative data not previously reported. Medical students began reporting reasons why neurology is difficult for them, which allowed the measurement of broad, encompassing qualitative data. It allowed students to 
clearly explain why they find neurology difficult. Overall, this influenced future neurophobia scale development in which researchers now commonly include at least one open-ended response to capture similar data from medical students.

The scale developed by McCarron et al. (2014) fills a literature gap in the reported reliability of neurophobia scales. McCarron et al. (2014) reported the internal consistency of their scale items developed from items in the previous scales (Schon et al., 2002; Flanagan et al., 2007; Zinchuk et al., 2010) as a Cronbach's $\alpha=0.73$. Cronbach's $\alpha$ is measured on a scale from 0 to 1 and $\alpha=0.73$ represents moderate internal consistency. A Cronbach's $\alpha$ closer to 1 represents more items within a scale have more shared covariance, meaning they are more likely to be measuring the same underlying construct (Cronbach, 1951). It is promising that the scale items previously developed are most likely measuring the same construct which McCarron et al. (2014) report is "a reliable finding of neurology's poor rating" (p. 3).

Subsequently, Pakpoor et al. (2014) incorporated two additional items along with the previous neurophobia scale items (Schon et al., 2002; Flanagan et al., 2007; Zinchuk et al., 2010). The two additional items were attempting to measure neurophobia by asking respondents to rate, on a 5-point Likert scale, their comfort in examination and comfort in developing a differential diagnosis of different medical specialties. Pakpoor et al. (2014) improved the original Schon et al. (2002) item that only measured a medical student's confidence in performing a physical examination (in different medical specialties). The original item may be capturing a medical student's deficiencies in their physical examination skills and not their comfort, or anxiety level, with the subject itself. Adding the word comfort potentially broadened the scope of this item and captures the 
medical student's feelings of ease, or potential lack of ease, with a subject. The authors theorize this lack of ease in a subject may consequently reflect anxiety. Further investigation on how the lack of ease in a subject directly measures the anxiety level of a subject was not explored in this study.

Shiels et al. (2017) described adding one question to their instrument, developed from previous studies (Schon et al., 2002; Flanagan et al., 2007; Zinchuk et al., 2010), to directly measure the level of neurophobia. The one additional question asks respondents to, Rank your agreement with the following statement: I have an aversion to neuroscience on a 5-point Likert-scale. This one item may be capturing neurophobia through the phrase aversion to neuroscience. It is plausible, however, the meanings of the words aversion and neuroscience may be misinterpreted by respondents. The respondent may be averse to neuroscience from disliking the subject, not because of fear related to neuroscience that would need to be present to measure neurophobia. Secondarily, the respondent may fear neuroscience, the molecular detail of neurons, and alternatively enjoy learning neuroanatomy, the anatomical connectivity within the brain and spinal cord. Additionally, the neurophobia construct might not be measurable by only one question item. A novel scale to measure a construct should have enough items to adequately encompass it and potentially include other scales to assess its initial validity and reliability.

Another issue related to measurement of this neurophobia construct as neuroanxiety is our inability to assess student's general anxiety level on a validated scale, namely the GAD-7 (Spitzer et al., 2006). Ideally, correlation of scores on the GAD-7 (Spitzer et al., 2006) with the NAS would provide convergent validity and allow 
researchers to control for medical students with general anxiety disorder when assessing neuroanxiety levels. Additionally, since many medical students are high-achieving students it could be theorized that neuroanxiety may be representing generalized anxiety disorder in high-achieving students learning neuroanatomy. Allowing researchers to measure both generalized anxiety disorder and neuroanxiety concurrently would allow further exploration into whether or not neuroanxiety is separate and distinct from generalized anxiety disorder. If it is found neuroanxiety is, perhaps, generalized anxiety disorder in medicals students learning neuroanatomy, further access and integration of mental health care in medical students may be warranted to treat medical student anxiety. One measurement limitation in our study with using a mental health screening tool, GAD-7 (Spitzer et al., 2006), is the lack qualified mental health professionals on the research team. For that rationale, the GAD-7 (Spitzer et al., 2006) was not administered along with the NAS.

In summary, medical education researchers have improved upon the original Schon et al. (2002) neurophobia scale by adding items to investigate reasons why students find neuroanatomy difficult (Flanagan et al., 2007) and providing openresponses to collect qualitative data on why neurology is difficult (Zinchuk et al., 2010). Additionally, researchers used the words comfort (Pakpoor et al., 2014) and averse (Shiels et al., 2017) to clarify the original perceived level of difficulty from the Schon et al. (2002) scale in efforts to capture fear and anxiety towards neurology. McCarron et al. (2014) added to the breadth of neurophobia scale development literature by reporting an internal consistency of Cronbach's $\alpha=0.73$. However, the scales are incorporating many medical specialties and are lacking focus only on the subject of neurology. The terms 
neurology, neuroanatomy, and neuroscience are used interchangeably in these studies and do not provide respondents clear definitions of these terms. Additionally, a literature search to-date found no psychometrically validated scales, outside of the McCarron et al. (2014) scale, measuring neurophobia. This present study will fill this gap in the literature by providing a theoretical framework for neuroanxiety, develop a novel instrument to measure it, and provide data for initial validity and reliability evidence for the instrument. Developing a scale to measure neuroanxiety will allow researchers to measure medical student neuroanxiety and potentially assess the efficacy of learning strategies or interventions designed to address it. Scale development methods used in this study are described below.

\subsubsection{Methods.}

Study Population. First through fourth year medical students are the population for the scale development portion of this study; second through fourth year medical students are the target population for finding the best predictors of neuroanxiety with multiple linear regression analysis. A convenience sample of medical students enrolled at a large, public, Southeastern university were invited to participate in the online survey administered in Qualtrics ${ }^{\circledR}$ in 2018. A second cohort of first-year medical students were invited to participate in the study in the Fall 2019 semester.

Data Collection Procedures. The director of the integrated first semester medical school course sent an email invitation to participate in the online survey twice during the Fall 2018 and Fall 2019 semesters. First-year medical students were invited to complete the 
survey at the beginning of the semester (pre-course) and again at the end of the semester (post-course). Ideally, two data points would be collected for each first-year medical student. Students were asked to provide a 5-digit code (suggested the last 5 digits of the student's phone number) for matching pre-course and post-course surveys. Upper-class medical students in 2018 were invited to participate in the online survey once at the end of the Fall 2018 semester. Completed surveys were exported from Qualtrics into a csv file in Microsoft Excel $^{\circledR}$. IP addresses were deleted from the files to protect anonymity. The files were imported into IBM SPSS ${ }^{\circledR}$ Version 26 where missing or non-complete surveys were deleted prior to analysis. Scales were coded and scored as described below.

Item Development of Neuroanxiety Scale (NAS). An existing published scale to measure general anxiety level, the GAD-7 (Spitzer et al., 2006), was chosen as a model from which to structure the NAS scale (Appendix B). One strength of the GAD-7 (Spitzer et al., 2006) is that it is a short, quick screening tool for generalized anxiety disorder that can be completed without the presence of a mental health professional. It can be completed quickly in a busy environment. However, it is only a screening tool for one type of anxiety disorder and a high score requires follow up with a mental health professional for further diagnosis. Overall, the GAD-7 (Spitzer et al., 2006) was chosen to model our scale after due to its high internal consistency $(\alpha=0.92)$ and test-retest reliability ( $r=0.83$ ) (Spitzer et al., 2006). Validity testing of the GAD-7 (Spitzer et al., 2006) scores against scores from two other validated anxiety scales showed strong correlations, Beck Anxiety Inventory (BAI) $r=0.72$ (Beck et al., 1998) and the Symptom Checklist-90 (SC-90) $r=0.74$ (Evenson et al., 1980). The BAI (Beck et al., 1998) is an 
established mental health screening tool for anxiety disorders. The SC-90 (Evenson et al., 1980) is a diagnostic mental health tool for disorders leading to psychological distress; anxiety disorder is one of the subscale measures considered to cause psychological distress. This suggests that a score on the GAD-7 (Spitzer et al., 2006) is measuring the same construct as the BAI (Beck et al., 1998) and the SC-90 (Evenson et al., 1980). Implications for this study suggest modeling our scale after an anxiety scale with strong validity and reliability evidence will aim to measure a form of anxiety. In our case, we aim to measure anxiety towards learning neuroanatomy in medical school.

Respondents were asked to subjectively rate how often they were bothered by the following symptoms during the past two weeks while studying neuroanatomy, during neuroanatomy lecture or while performing a neurological exam $(0=$ Not at All, $1=$ Several Days, 2= More Than Half the Days and 3= Nearly Every Day). These items encompass the scope of the operational definition of neuroanxiety: fear of neuroanatomy, ability to learn neuroanatomy and its clinical applications. Care with wording items to reflect the three dimensions of anxiety with physiological, cognitive, and behavioral factors (Lang, 1971; Lang, 1978) was taken (see Appendix B NAS Instrument Modifications for further information). Scoring of the NAS, adapted from the GAD-7 (Sptizer et al., 2006), is reported as a sum total of raw scores where significantly higher scores represent greater self-reported neuroanxiety and lower scores represent lower self-reported neuroanxiety.

Exploratory Factor Analysis. An exploratory factor analysis (EFA) with principal axis factoring will be used to explore the underlying factor structure of the NAS instrument using the entire sample population. EFA is recommended over a confirmatory factor 
analysis for initial scale development since it is possible our hypotheses regarding the latent construct's factor structure may not be correct (Carpenter, 2018). Principle axis factoring, assuming a causal model which generalizes to the population exists (Mabel \& Olayemi, 2020), was chosen over a principal components analysis which is typically used to reduce the number of items in a scale (Abdi \& Williams, 2010).

A Bartlett's test of sphericity and the Kaiser-Meyer-Olkin test (KMO) will be conducted to determine if the data for the 7-item NAS are suitable for exploratory factor analysis (EFA). A significance level of $p<0.01$ for the Bartlett's test of sphericity (Bartlett, 1950) and a KMO > 0.6 will suggest the proportion of variance of the items are due to an underlying factor- which makes a factor analysis useful for these data. Several measures will be used to determine the number of factors to retain for this scale: Eigenvalues greater than 1 according to the Kaiser-Guttman rule (Kaiser, 1991), the elbow of the scree plot, and parallel analysis with principal components analysis $\left(95^{\text {th }}\right.$ percentile criterion). If applicable, the following criteria will be used for retaining factors: (1) minimal pattern coefficients of 0.4 ; (2) nonrelevant factor pattern coefficients are less than 0.3 ; and (3) nonrelevant factor pattern coefficients were 0.2 less than the pattern coefficient on the retained factor (Thompson, 2004; Shuck et al., 2017). One factor is anticipated based on the principal component analysis (PCA) results from which the NAS scale was derived, the GAD-7 (Spitzer et al., 2006). PCA on a 15-item scale combining the 7-item GAD-7 (Spitzer et al., 2006) instrument and the 8-item Patient Health Questionnaire (PHQ-8) (Kroenke et al., 2001) instrument identified the seven items from the GAD-7 (Spitzer et al., 2006) scale loaded onto one factor with an eigenvalue greater 
than 1; PCA confirmed the eight depression items, the PHQ-8 (Kroenke et al., 2001), loaded onto a separate factor (Spitzer et al., 2006).

Instruments for initial validity analysis. In addition to the 7 NAS items, the development of three additional instruments is described below. The purpose of administering scales, concurrently with the NAS, will allow initial validation of this novel instrument. These instruments aim to measure neuroanatomy beliefs, emotions towards neuroanatomy, and neuroanatomy self-efficacy.

Scales specific to measuring neuroanatomy beliefs and emotions, and neuroanatomy self-efficacy, have not yet been explored in the research. Previous studies on recognizing emotion of language (Whissell,1989; Whissell, 2009) informed item development of the neuroanatomy beliefs and emotions scale items. Items developed for the neuroanatomy self-efficacy scale were informed by an anatomy self-efficacy scale (Burgoon et al., 2012); neuroanatomy self-efficacy scale development is described in Chapter 4. Neuroanatomy beliefs, emotions towards neuroanatomy, and neuroanatomy self-efficacy will be correlated with NAS scores to provide convergent validity evidence.

Item Development of Neuroanatomy Beliefs Scale. This scale contains three, openresponse items requiring students to provide three beliefs they have about the field of neurology and neurologists. It was decided against providing a series of common negative neurology beliefs and neurologist stereotypes, for which students would score on a Likert scale, to minimize both response bias and survey fatigue. Keeping a neutral tone of survey items prevents leading students into providing answers, students believe, 
the researchers may want to hear. Having students provide open responses may prevent survey fatigue from the other $(n=20)$ response items within the instrument. Scoring emotional words is based on the circumplex model of human affect which proposes emotional states arise from two main dimensions: valence (level of pleasantness) and arousal (level of alertness or activation) (Russell, 1980; Barrett, 2004; Posner et al., 2005). A literature search found the Dictionary of Affect in Language (DAL) (Whissell, 1989; Whissell, 2009) provides an evaluation (pleasantness) and arousal (activation level) score for emotional words based on a 3-point scale in both the evaluation $(1=$ unpleasant, $2=$ in-between, $3=$ pleasant $)$ and arousal $(1=$ passive, $2=$ in-between, $3=$ active $)$ dimensions. The concurrent validity reported for the 2009 revised DAL is $r=0.71$ for evaluation and $r=0.45$ for activation based on words from the original dictionary (Whissell, 1989); test-retest reliability correlations were $r=0.80$ for evaluation and $r=$ 0.69 for activation (Whissell, 2009). The total score is reported as an average of evaluation and activation scores for all three words provided by respondents. A lower score suggests the respondent had more fear-based beliefs towards neurology, whereas higher scores correspond to higher positive beliefs towards neurology and neurologists.

\section{Item Development of Emotions Towards Neuroanatomy Scale. The Emotions}

Towards Neuroanatomy scale contains three, open-response items requesting students to self-reflect on how they felt about neuroanatomy at the start of medical school and to provide three strongest emotions they felt during that time. Similar to the neuroanatomy beliefs scale, providing an encompassing list of common positive and negative emotions, for which students would score on a Likert scale may lead to survey fatigue. 
Alternatively, if a student feels an emotion that is not on the list may lead to inaccurate reporting or response bias. Scoring emotional words is based on the circumplex model of human affect which proposes emotional states arise from two main dimensions: valence (level of pleasantness) and arousal (level of alertness or activation) (Russell, 1980; Barrett, 2004; Posner et al., 2005). The researcher assigns a 3-point score for evaluation $(1=$ unpleasant, $2=$ in-between, $3=$ pleasant $)$ and arousal $(1=$ passive, $2=$ in-between, $3=$ active) dimensions for each word provided by the student based on the Dictionary of Affect in Language (DAL) (Whissell, 1989; Whissell, 2009). The concurrent validity reported for the 2009 revised DAL is $r=0.71$ for evaluation and $r=0.45$ for activation based on words from the original dictionary (Whissell, 1989); test-retest reliability correlations were $r=0.80$ for evaluation and $r=0.69$ for activation (Whissell, 2009). The total score is reported as an average of evaluation and activation scores for all three words provided by respondents. A lower score suggests the respondent felt more fearful, or negatively, towards neuroanatomy at the beginning of medical school and higher scores suggests respondents felt excited, or more positive, towards neuroanatomy at the start of medical school.

Item Development of Neuroanatomy Self-Efficacy Scale. The thirteen subjective items that comprises this subscale represents a person's beliefs in his or her own abilities to successfully complete a neuroanatomy task, based Bandura's Social Cognitive Theory (1989). These thirteen items were developed and scored based on the 16-item anatomy self-efficacy scale developed by Burgoon et al. (2012) (Appendix B). Principal Axis Factoring of the original 16-item scale found one factor, anatomy self-efficacy, with an 
internal consistency (Cronbach's $\alpha$ ) between 0.90 to 0.96 for all sixteen items (Burgoon et al., 2012). We modified this scale for use as a neuroanatomy self-efficacy scale and the development of the NSES scale providing initial validity and reliability evidence is described in Chapter 4. Respondents were asked to rate their confidence level on a 5point Likert scale in doing 13 different neuroanatomy related tasks (1= Not at All Confident, $2=$ Only a Little Confident, $3=$ Fairly Confident, $4=$ Very Confident and $5=$ Totally Confident). Neuroanatomy self-efficacy is scored as raw scores reported as a sum score of all items where lower scores signify lower self-reported neuroanatomy selfefficacy while higher scores represent greater self-reported neuroanatomy self-efficacy.

Pilot. A pilot of 10 medical student volunteers was conducted to assess content validity, online administration functionality in Qualtrics ${ }^{\circledR}$, and timing the length of the survey. Respondents were asked for their feedback assessing content validity: grammar, language, wording and question sensitivity. The pilot test assessed the length of time for pilot survey participation for informing participants of the survey's anticipated time commitment. Students responded favorably to wording of question items during the pilot assessment and no edits to language or grammar of scale items were suggested by respondents. The online administration in Qualtrics ${ }^{\circledR}$ functioned without issue and the average time to complete the survey was 12 minutes.

Internal reliability and validity analysis. The internal consistency reliability for the 7item scale will be examined by calculating Cronbach's alpha and a Guttman split-half reliability coefficient (Guttman, 1946; Cronbach, 1951; Clark \& Watson, 1995). For 
items that group together onto factors, subscale Cronbach's alphas for grouped items for each factor will be examined. The recommended acceptable 0.8 level for internal consistency will be used for all analyses (Cronbach, 1951; Clark \& Watson, 1995).

We will examine relationships (correlations) between the novel NAS scale with other scale scores to further provide validity evidence. Convergent validity assesses how well the score of other scales theoretically measures this same construct (neuroanxiety) concurrently. The scores of the neuroanatomy beliefs, neuroanatomy emotions, and neuroanatomy self-efficacy scales will be correlated with the novel NAS scores. Higher scores of the neuroanatomy emotions and neuroanatomy beliefs subscales represent less fear-based, anxious emotions and beliefs while higher NAS scores reflect greater anxiety towards learning neuroanatomy; therefore, a negative correlation between NAS scores and neuroanatomy emotions and neuroanatomy beliefs subscales is anticipated. Negative emotions, particularly anxiety, and fear-based beliefs have been shown to be negatively correlated with student self-efficacy in the literature (van Dinther et al., 2011; Barrows et al., 2013; Razavi et al., 2018). A negative correlation between neuroanxiety and neuroanatomy self-efficacy is expected based on previous literature demonstrating an inverse relationship between fear-based emotions and self-efficacy.

\subsubsection{Results.}

3.2.4.1 Participants and response rate. All medical students enrolled at a large, public, Southeastern university ( $n=625$ ) were invited to participate in the online survey administered in Qualtrics ${ }^{\circledR}$ in 2018. A second cohort of first-year medical students ( $n=$ 162) were invited to participate in the study in the Fall 2019 semester. A total of 280 
medical students voluntarily participated with a $35 \%$ response rate. The sample was comprised of $48.6 \%(n=135)$ female and $44.2 \%(n=123)$ male respondents; $7.2 \%(n=20)$ self-identified as non-binary $(n=1)$ or did not provide gender information $(n=19)$. Of the respondents, $60.1 \%(n=169)$ were first-year and $39.9 \%(n=111)$ were upper-class (second-, third- or fourth-year) medical students. A small number $(n=47)$ first-year medical students completed both pre-course and post-course surveys, and entered their 5digit code, for matching data analysis.

The study drew from a total student population of 787 medical students, $55 \%$ male and $45 \%$ female, 23 of which were undergraduate neuroscience majors $(2.92 \%)$ (see Table 3.3). Publicly available data from the University of Louisville School of Medicine show the average age of all five entering classes $(2015-2019)$ was 23.2. The average undergraduate overall GPA was 3.68 with the average Biology Chemistry Physics and Math (BCPM) GPA being 3.60. Racial demographics, reported as underrepresented in medicine, comprised $11 \%$ of entering students. It should be noted that the entering class of Fall 2017 had the lowest racial diversity (6\% underrepresented in medicine) while the Fall 2015 entering class profile comprised the highest diversity (14\%). The average age of the entering classes from 2015 to 2019 appears to decrease slightly from 24 to 23 , with the Fall 2019 with the youngest average age of 22.

3.2.4.2 Descriptive statistics. Chi square tests were used to examine the gender differences between the participants who completed the instrument and the general population of medical students at the university. Chi square tests indicated there were no differences in gender between first-year medical student participants and the entering 
first-year classes in both the Fall 2018, $X^{2}(1, n=30)=0.54, p=0.46$, and Fall 2019, $X^{2}(1, n=107)=1.7, p=0.19$, semesters. A chi square test also showed no differences in gender between upper-class medical students who completed the survey compared to enrolled upper-class medical students in $2018, X^{2}(1, n=111)=2.3, p=0.13$. Incomplete surveys $(n=45)$ were dropped from the analysis resulting in a total of $n=233$ surveys for data analysis. The sample was comprised of $48.56 \%(n=119)$ female, $44.24 \%(n=113)$ male respondents, and ( $n=1)$ non-binary gender student. The non-binary gender student was given their own category, however not included in the binary gender analysis.

3.2.4.3 Exploratory Factor Analysis (EFA). Initially, the factorability of the 7-item NAS scale was examined. The results of measuring sampling adequacy using Bartlett's test of sphericity $\left(\chi^{2}=1144.92, d f=21, p<0.001\right)$ and the Kaiser-Meyer-Olkin test (0.902) suggests the data were suitable for EFA. Exploratory factor analysis (EFA) results are shown in the factor matrix and extraction communalities (Table 3.5), eigenvalues and scree plot (Figure 3.1). In Figure 3.1, the scree plot suggests factors to the left of the sharp bend, or elbow, are considered significant and should be retained.

The factor matrix demonstrates a high correlation between each of the scale items and the factor resulting in a one factor solution for the NAS scale (Table 3.5). The factor matrix for scale items ranges from 0.62 for Item 5 to 0.84 and 0.85 for Items 3 and 2, respectively. Item 5, "being so restless that it's hard to sit still" reflects physiological aspects of anxiety. The directions for the scale reminded the respondent to take into consideration being in a neuroanatomy classroom or clinical setting, however this item does not mention neuroanatomy. Unlike Items 3 and 2, which showed much stronger 
factor loadings, Item 3 mentions "not being able to focus on neuroanatomy" and Item 2 describes "thinking neuroanatomy is too difficult". Similarly, the proportion of variance in the items explained by the factor $\left(h^{2}\right)$ was higher for Items 3 and $2(0.7$ and 0.72$)$ than for Item 5 (0.39). A parallel analysis also suggests a one factor solution with one eigenvalue greater than one explaining $57.4 \%$ of the variance. The factor analysis results suggest the NAS is a brief scale that reflects one construct.

3.2.4.4 Internal consistency, validity, and reliability. The Cronbach's alpha of the 7item NAS $(\alpha=0.91)$ is above the recommended acceptable 0.8 level (Cronbach, 1951; Clark \& Watson, 1995) for internal consistency. A Guttman split-half reliability coefficient was calculated to be 0.86 (Guttman, 1946).

The following scales were included in survey administration to assess convergent and discriminant validity of the novel NAS scale: neuroanatomy beliefs, emotions towards neuroanatomy, and neuroanatomy self-efficacy scale (NSES). Convergent validity was calculated by correlating the NAS scores with neuroanatomy beliefs and emotions towards neuroanatomy scales. These scales are scored to reflect more positive, less anxious and fearful emotions as higher scores and lower scores reflect more anxious and fearful emotions; therefore, a negative correlation is anticipated for convergent validity. A significant negative correlation between neuroanxiety and each of the subscales (emotions towards neuroanatomy $r=-0.55, p<0.0001$; beliefs towards neuroanatomy $r=-0.25, p<0.0001$ ) was observed. Discriminant validity was measured using the NSES scale, measuring a student's neuroanatomy self-efficacy. A higher NSES score reflects greater confidence in completing neuroanatomy specific tasks. A negative 
correlation is anticipated between these negatively correlated constructs (educational anxiety and educational self-efficacy) as reported in the literature (van Dinther et al., 2011; Barrows et al., 2013; Razavi et al., 2018). A negative correlation ( $r=-0.33, p<$ 0.0001) was observed.

3.2.4.5 Neuroanxiety Scores. The overall neuroanxiety score of all our medical student respondents $(n=280)$ was, $M=4.99, S D=4.56$. Scoring of respondents is based on the scale from which it was developed, the GAD-7 (Spitzer et al., 2006). Scores of $\geq 6$ on the GAD-7 (Spitzer et al., 2006) are considered to be exhibiting signs of anxiety. Respondents with higher scores $(\geq 6)$ will be considered as exhibiting neuroanxiety. Higher scores represent greater neuroanxiety while lower scores represent less neuroanxiety. Approximately $40 \%$ ( $n=113)$ of our respondents reported experiencing neuroanxiety in medical school with NAS scores $\geq 6$.

Neuroanxiety and medical school class. Interestingly, neuroanxiety levels differed significantly between first year medical students and upper-class medical students, $t(278)=4.03, p<0.001$, with a medium effect size (Cohen's $d=0.49)$ indicating a moderate practical significance (Figure 3.2). Our results were consistent with the Shiels et al. (2017) study; first year medical students overall exhibited low neuroanxiety, $M=$ 4.12, $S D=4.33, n=169$, with $31.95 \%$ ( $n=54)$ of students reporting neuroanxiety with scores $\geq 6$. Upper-class medical students reported exhibiting significantly higher neuroanxiety, $M=6.31, S D=4.63, n=111$, with $58.5 \%(n=65)$ showing neuroanxiety scores $\geq 6$. 
Neuroanxiety and gender. An independent $t$-test showed female medical students reported higher levels of neuroanxiety than their male counterparts, $t(230)=3.92, p<$ 0.001 , with a medium effect size (Cohen's $d=0.52)$ indicating a moderate practical significance (Figure 3.3). Female medical students reported on average $(M=6.16, S D=$ $4.56, n=119)$ nearly double the neuroanxiety than male participants $(M=3.89, S D=4.24$, $n=113)$.

To further investigate this relationship, a factorial analysis of variance (ANOVA) was conducted to compare the main effects of gender and previous neuroscience experience and the interaction effect between gender and previous neuroscience experience on neuroanxiety levels. As anticipated, the main effect analysis showed females demonstrated significantly higher levels of neuroanxiety $(p<0.001)$. However, there was no main effect for previous neuroscience experience on neuroanxiety ( $p=$ 0.064) and no interaction effect between gender and previous neuroscience experience $(p=0.067)$ on neuroanxiety levels. However, additional analyses suggest that the data line is trending towards female students without any premedical neuroanatomy and/or neuroscience experience reporting greater neuroanxiety levels than their female classmates with previous neuroanatomy and/or neuroscience experience, although the results are not statistically significant (Figure 3.4). Interestingly, even female medical students with previous neuroscience experience have increased neuroanxiety scores than their male classmates without previous experience (Figure 3.4). Of students reporting any premedical exposure to neuroscience or neuroanatomy before starting medical school, 
$47.3 \%$ were male and $52.7 \%$ were female; male students did not have significantly more premedical neuroscience and/or neuroanatomy exposure, $X^{2}(2, n=232)=1.52, p=0.47$.

\subsection{STUDY 2 PREDICTORS OF NEUROANXIETY}

3.3.1 Study Purpose. The purpose of this second study is to investigate variables that predict neuroanxiety in a medical student population. Kam et al. (2013) reported three main predictors of neurophobia in medical students: female gender, low neuroanatomy interest, and low neuroanatomy knowledge in a multivariate analysis. Other factors contributing to neurophobia have been explored in the research with neuroanatomy difficulty level and complex physical exam (Youssef, 2009) with poor teaching experiences identified as major contributors (Schon et al., 2002; Abulaban et al., 2015). Inadequate teaching is a consistent theme with neurophobia research (Schon et al., 2002; Flanagan et al, 2007; Youssef, 2009; Zinchuk et al., 2010; Abulaban et al., 2015; Javaid et al., 2018). Yet, details about these teaching experiences and, alternatively, instructional strategies that could improve neuroanatomy instruction are lacking in the literature. The second study in this chapter aims to fill this literature gap by exploring specific instructional predictors of neurophobia (neuroanxiety). This study will add to the existing literature investigating gender and neuroanatomy knowledge predicting neurophobia (neuroanxiety). Exploring specific instructional predictors of neuroanxiety will inform the curriculum development and learning strategies designed minimize the effects of neuroanxiety. The predictors of neuroanxiety found in this study will inform the development of an eLearning intervention designed to minimize its effects Chapter 5 of this dissertation. 


\subsubsection{Background}

Predictors of neuroanxiety. A review of the literature reveals inadequate, even described as poor, teaching as a key contributor to neuroanxiety (Flanagan et al, 2007; Youssef, 2009; Zinchuk et al., 2010; Abulaban et al., 2015). Medical education research studies reveal neuroanatomy teaching is rated as moderate to poor (Flanagan et al., 2007) and neuroanatomy instructors themselves are consistently described as inadequate or needing improved teaching skills (Zinchuk et al., 2010; Abulaban et al., 2015) leading many medical students (70\%) to report their neurophobia is the result of a bad teaching experience (Abulaban et al., 2015). Yet, specific descriptions of bad teaching experiences and, alternatively, instructional strategies that could improve neuroanatomy instruction are not provided. This study aims to contribute depth to the existing literature by examining specific instructional deficiency variables (i.e., lecture, laboratory, 3D models, clinical examples, and learning objectives) with other variables retrieved from the literature (i.e., gender, previous experience, and reputation) in relation to neuroanxiety. A review of the predictor variables of gender, previous neuroscience experience, instructional deficiencies (i.e., lecture, laboratory, 3D models, clinical examples, and learning objectives), and neuroanatomy reputation in relation to neuroanxiety are presented.

Gender (G). Female gender has been described in the literature as a possible contributor of neurophobia (Kam et al., 2013; Pakpoor et al., 2014; Anwar et al., 2015). One study of both medical students and residents $(n=158)$ reported female gender increases the risk of 
neurophobia (Kam et al., 2013). Another large-scale study of medical students $(n=2877)$ at 24 medical schools in the United Kingdom suggests female medical students are less likely to choose a neurology career possibly due to neurophobia (Pakpoor et al., 2014). Similarly, another study investigating gender and specialty choice found female medical students are choosing neurology (5.4\% vs $7 \%)$ and neurosurgery (5.3\% vs $7.3 \%)$ at a lower rate than their male counterparts (Ng-Sueng, 2016). The American Academy of Neurology created a Gender Disparity Task Force (GDTF) to address the neurology gender gap (AAN, 2017). Increasing the number of female neurological specialists could help supplement the current shortage of neurologists and neurosurgeons in the United

States while concurrently narrowing the gender gap. It is unknown whether neurophobia may be differentially affecting female medical students, thereby preventing them from pursuing a neurology career. It is, therefore, important to study female gender as a predictor of neuroanxiety.

\section{Premedical neuroanatomy and/or neuroscience experience (PNE). Premedical}

experiences have been described in the literature to benefit first-year medical student success (Foreseter et al., 2002; Peterson \& Tucker, 2005; Kondrashov et al., 2017). Premedical experiences, specifically with a cadaveric dissection laboratory component, are positively correlated with medical school grades and GPA in medical gross anatomy courses. Forester et al. (2002) surveyed first-year medical students $(n=440)$ and found those with premedical gross anatomy with cadaveric specimens showed higher anatomy grades than students with premedical anatomy experience lacking cadaveric specimens. Peterson and Tucker (2005) found students with premedical anatomy laboratory 
dissection experience is a predictor of GPA and moderately correlates with gross anatomy grades in medical school. Similarly, Kondrashov et al. (2017) found medical students having experience with premedical cadaveric specimens provided the greatest perceived benefit for medical school gross anatomy success. No studies were found, todate, reporting premedical neuroanatomy and/neuroscience experience and medical student success. This is an important literature gap to address, especially with less medical students choosing neurology as a specialty (AAN, 2017; Gutmann et al., 2019) and the concurrent a shortage of neurological specialists (AAN, 2013; AMA 2014; Hasan et al., 2019).

Interestingly, a large-scale study $(n=51,816)$ identified characteristics of students that chose to pursue neurology $(n=1,920)$ (Gutmann et al., 2019). Analysis of the Association of American Medical Colleges (AAMC) Matriculating Student Questionnaires (2013 - 2017) found students who rated their basic neuroscience course as excellent, or majored in neuroscience, were more likely to pursue neurology (Gutmann et al., 2019). Gutmann et al. (2019) demonstrate the importance of positive educational experiences early in the basic medical neuroscience curriculum, or even before entering medical school, on later choosing a neurology career.

Data from our discussion group, in Chapter 2 of this dissertation, suggest students with premedical neuroanatomy experiences felt more comfortable, and therefore, potentially exhibit less neuroanxiety. For example, a student quote from the Chapter 2 study yielded: If I hadn't seen it (neuroanatomy) before... I would not have felt comfortable doing that (neuroanatomy) at all. Our findings, combined with the Gutmann et al. (2019) study, suggest positive educational experiences early in the basic medical 
neuroscience curriculum may increase student comfort level, possibly reducing neuroanxiety. It is unknown whether having premedical experience in neuroscience or neuroanatomy predicts lower neuroanxiety in medical students without any premedical experience.

Instructional Deficiency: Lecture (Lec). Didactic neuroanatomy lecture experiences are documented as one contributor of neurophobia (Youssef, 2009; Abulaban et al., 2015; Javaid et al., 2018). Additionally, students report decreased lecture time has contributed to neurophobia (Flanagan et al., 2007; Youssef, 2009), especially with the decrease in lecture hours devoted to neuroanatomy instruction in recent years due to curriculum integration (Drake et al., 2002; Drake et al. 2009). Medical educators are exploring ways to improve neuroanatomy lecture experiences through flipped-classroom (Veeramani et al., 2015) and team-based learning exercises (Shiels et al., 2017) to address this issue. Veeramani et al. (2015) found $92 \%$ of medical students $(n=130)$ perceived the flipped classroom experience as providing a better understanding of the subject contributing to students feeling more comfortable with the material than didactic lecture. Although this study did not directly investigate the effect of the flipped classroom on neurophobia, it indicates a potential lecture approach worth pursuing. Shiels et al. (2017) found casebased teaching (CBT) reduced overall neurophobia, which also indicates a potential educational strategy worth pursuing to potentially mitigate neurophobia.

Medical educators at our institution have incorporated problem-based learning (PBL) and flipped-classroom exercises within the neuroanatomy curriculum to both improve the lecture experience and potentially reduce neuroanxiety. However, our 
discussion group data from the qualitative study in Chapter 2 of this dissertation suggest that didactic lectures may contribute to neuroanxiety. Students reported the organization of long lectures spaced too closely together (Table 2.4) as contributing to neuroanxiety. The instructional team often overestimated student's base neuroanatomy knowledge during didactic lectures, which also contributed to neuroanxiety at our institution (Table 2.3). It is unknown if these lecture experiences at our institution predict neuroanxiety, and therefore, is an important variable to investigate.

Instructional Deficiency: Lab (L). Cadaveric laboratory experiences in neuroanatomy have been reported in the literature to benefit both test scores and long-term retention (Macchi et al., 2007; Rae et al., 2016) and even increased scores on the $\mathrm{NBME}^{\circledR}$ Subject Examination (Rae et al., 2016). Macchi et al. (2007) compared the long-term retention of second-year medical students $(n=40)$ who performed brain dissection to students $(n=40)$ who did not. Students who completed the brain dissections scored higher on the post-test examination (57\%) than the non-dissection control group (43\%). Interestingly, one year later the dissection group performed much better (65\%) overall compared to the nondissection control group (40\%) (Macchi et al., 2007). Similarly, Rae et al. (2016) performed a study comparing students in dissection $(n=80)$ and non-dissection groups ( $n=85)$ and reported higher post-test examination scores from students in the dissection group. Additionally, students were followed longitudinally and their scores on the $\mathrm{NBME}^{\circledR}$ Subject Examination were compared. Students who participated in the dissections scored significantly higher on the $\mathrm{NBME}^{\circledR}$ Subject Examination one year later. These studies suggest cadaveric laboratory dissection may improve neuroanatomy 
learning and increase long-term retention. It is unknown, however, whether neuroanatomy cadaveric laboratory experiences influence neuroanxiety.

Neuroanatomy laboratory prosections, identifying anatomical structures on already cut and dissected specimens, comprise the neuroanatomy laboratory component of the curriculum at our institution. The qualitative data from the discussion group in Chapter 2 of this dissertation identified neuroanatomy laboratory instruction as a potential source of neuroanxiety. Our students reported frustration with the instruction and faculty inconsistencies in the neuroanatomy laboratory (Table 2.3). The neuroanatomy faculty rotating within the neuroanatomy laboratory were described by students as not getting a consensus (from the faculty) all the time. These inconsistencies were described as contributing to students feeling frustrated and overwhelmed while learning neuroanatomy (Table 2.5). While the literature report neuroanatomy laboratory experiences positively influence student learning through test-scores and long-term retention (Macchi et al., 2007; Rae et al., 2016), it is hypothesized neuroanatomy laboratory will be a predictor of neuroanxiety due to the inconsistent instruction reported by students at our institution.

Instructional Deficiency: 3D models (3D). Medical students have reported the lack of 3D models to aid in visualization of neuroanatomy as another contributor of neurophobia (Youssef, 2009; Abulaban et al., 2015; Javaid et al., 2018). Interestingly, the literature supports this student perspective; studies investigating student learning in neuroanatomy using 3D models show increased test scores when compared to 2D instruction (Estevez et al., 2010; Kockro et al., 2015; Allen et al. 2016). Estevez et al. (2010) compared overall 
quiz scores of first-year medical students $(n=50)$ receiving the $3 \mathrm{D}$ model compared to first-year medical students $(n=51)$ receiving traditional 2D instruction. Students in the 3D model group performed better on quiz scores, particularly when 3D specific questions were isolated. In a separate study, Kockro et al. (2015) randomly assigned second-year medical students to receive a lecture $(n=80)$ and a (3D) animated tour $(n=89)$ of the third ventricle. Not only did students who received the 3D animation score higher on the posttest, they also perceived the 3D animation as beneficial to spatial orientation. A preliminary study investigating gender differences of spatial orientation of medical students $(n=70)$ used a spatial orientation test (Mental Rotation Test) reported women scored significantly lower than men with spatial orientation abilities (Akle et al., 2018). Additionally, Akle et al. (2018) have a future study planned to compare the effect of 3D models to $2 \mathrm{D}$ instruction in neuroanatomy learning and spatial orientation using the Mental Rotation Test. Results from this study could support the further development and implementation of neuroanatomy 3D eLearning tools.

Neuroanatomy instruction at our institution has limited access to 3D models for neuroanatomy. The data from our discussion group in Chapter 2 of this dissertation are consistent with the literature; our students described having more access to 3D models would be easier for translating where neuroanatomy landmarks are spatially located on a 2D image (Table 2.1). It is predicted that lacking 3D models in our curriculum will predict neuroanxiety in this study.

Instructional Deficiency: Clinical examples (CE). Both the difficulty level of neurological clinical cases (Schon et al., 2002) and the lack of clinical case integration in 
the neuroanatomy curriculum (Youssef, 2009; Abulaban et al., 2015; Javaid et al., 2018) are found to contribute to neurophobia. The neurology clinical examination is complex (Schon et al., 2002) and interpreting the results may be difficult for a novice learner of neuroanatomy. A study comparing a clinical case-based curriculum, assisting novice students to interpret these clinical examination results, with conventional teaching methods showed promising results. First-year medical students in a human neuroanatomy course were randomly assigned to a conventional group $(n=27)$ or a clinical case group $(n=58)$. The clinical case group performed $11 \%$ better than the conventional group on mid-semester benchmarks and $12 \%$ better on their final examinations (Greenwald and Quitadamo, 2014).

The neuroanatomy curriculum at our institution incorporates clinical cases as part of the curriculum, however it is not a case-based curriculum. Similar to medical students in the literature (Youssef, 2009; Abulaban et al., 2015; Javaid et al., 2018), student responses from the discussion group in Chapter 2 of this dissertation report not enough clinical content in the curriculum (Table 2.2). Additionally, students described the clinical content that was presented was too complex (Table 2.1), overwhelming (Table 2.5) and contributed towards neuroanxiety. Considering the literature and findings at our institution, it is hypothesized the lack of clinical-case examples in the curriculum will predict neuroanxiety at our institution.

Instructional Deficiency: Learning objectives (LO). Medical education literature is lacking evidence to support learning objectives significantly contribute to educational anxiety. However, learning objectives may be related to uncertainty and anxiety more 
globally. Learning objectives allow students to clearly know what is expected from them in a course. Grupe and Nitschke (2013) propose that uncertainty leads to anxiety through the Uncertainty and Anticipation Model of Anxiety (UAMA). This model proposes the neurobiology circuitry underlying why uncertainty, or not knowing what to expect, promotes maladaptive anxiety responses. One could hypothesize, therefore, that unclear neuroanatomy learning objectives could lead to neuroanxiety through student uncertainty. Student responses from our discussion group in Chapter 2 of this dissertation are consistent with the UAMA model (Grupe \& Nitschke, 2013). Students reported neuroanatomy learning objectives were unclear and not reflective of what the students were learning (Table 2.3). Students described not knowing what material to study, due to poorly written neuroanatomy learning objectives, contributed to being frustrated and overwhelmed when learning neuroanatomy (Table 2.5). This uncertainty, and not knowing what was expected of them in the neuroanatomy course, suggests it may contribute to anxiety towards learning neuroanatomy. This is the rationale behind our hypothesis that poorly written neuroanatomy learning objectives will predict neuroanxiety.

University of Louisville's neuroanatomy reputation (UL-REP). Neurology has a reputation for engendering low interest among medical students (Kam et al., 2013; Shiels et al., 2017) and is even reported as a predictor of neurophobia (Kam et al., 2013). The Kam et al. (2013) study found both medical students and residents $(n=158)$ who reported low interest in neurology exhibited greater neurophobia levels. Another study of first- 
and second-year medical students $(n=466)$ found $51 \%$ of students with reported low interest in neuroscience exhibited moderate to extreme neurophobia levels.

Neuroanatomy also has the reputation in the literature for being one of the most difficult medical specialties (Schon et al., 2002; Youssef, 2009; Abulaban et al., 2015). One survey of medical students $(n=345)$ found students perceived neurology as far more difficult than any other subject (Schon et al., 2002). Similarly, another study of fourthand fifth-year medical students $(n=167)$ found medical students rated neurology the most difficult subject (Youssef, 2009). Many (24\%) of these respondents assessed neurology difficulty as a 5, on a 5-point Likert scale, which was much higher than any other discipline in the study (Youssef, 2009). Another wide-scale survey of medical student ( $n=422)$ attitudes towards neurology was conducted at six different medical schools in Saudi Arabia (Abulaban et al., 2015). This survey reported most (85.5\%) participants perceived neurology as difficult (4) on a 4-point Likert scale. Historically, even neurologists seem to perpetuate the myth that "only young Einsteins" can succeed in neurology (Schon et al., 2002, p. 559).

Our data suggest that neuroanatomy's reputation at our university may be contributing to neuroanxiety. From our qualitative discussion group, we identified firstyear medical students reported statements such as, The second-years told me neuroanatomy was really overwhelming! Now I am worried out about neuro. Other firstyear medical students at our institution reported not believing they could complete the course or even wanting to drop out of medical school because the neuroanatomy portion was so difficult. Our qualitative findings are consistent with the literature suggesting neuroanatomy has a difficult reputation (Schon et al., 2002; Youssef, 2009; Abulaban et 
al., 2015). This study will explore whether neuroanatomy's reputation for being difficult at our institution will predict neuroanxiety.

\subsubsection{Methods}

Predictor Variables for Multiple Linear Regression Analysis. The eight predictor (independent) variables of gender, premedical neuroscience and/or neuroanatomy experience, lecture, laboratory, 3D models, clinical examples, learning objectives and the University of Louisville's neuroanatomy reputation will be explored to determine which variables explain a significant amount of the variability in neuroanxiety. The development of predictor variable items is described below.

Demographic Information (G). One demographic item, asking the respondent's gender, was informed by studies for inclusive measures of gender (Reisner et al., 2014; Bauer et al., 2017; Fraser, 2018). With $0.6 \%$ of the United States population (approximately 1.4 million adults) identifying as transgender (Flores et al., 2016), survey research has increasingly sought to include individuals that do not identify as a binary (male or female) gender. We relied on survey research in this area to inform our item development (Reisner et al., 2014; Bauer et al., 2017; Fraser, 2018) since there is currently no "gold standard" for gender inclusive survey items. We used the flow chart published in Fraser (2018) to determine the best practices for writing our gender item. Even though it was not necessary to identify transgender participants within our sample, we wanted to include non-cisgender survey items to respect our student diversity. The gender item provides an open response where respondents can self-describe their gender or prefer not to say. 
Premedical Neuroscience and/or Neuroanatomy Experience (PNE). One dichotomous item $(0=N o, 1=Y e s)$ differentiated between any premedical neuroanatomy and/or neuroscience experience $(1=Y e s)$ and none $(0=N o)$. This item is part of a five-item scale differentiating between premedical neuroscience and/or neuroanatomy experiences for use in data analysis in Chapter 4 with the novel Neuroanatomy Self-Efficacy Scale (NSES). Scoring of this scale for use with the present study was from item 5 of the Premedical Neuroscience and/or Neuroanatomy Experience Scale (Appendix B). One confirmatory question, item $5 \mathrm{~B}$, asked respondents to confirm their lack of neuroscience and/or neuroanatomy exposure in any capacity before starting medical school. Only students who scored No for item 5 and the confirmatory item 5B, ensuring no premedical experience, were scored as no premedical neuroanatomy and/or neuroscience experience $(0=N o)$.

Other Predictor Variables. Respondents were asked to rate the impact of each one of these six independent variables [Lecture (Lec), Laboratory (L), 3D Models (3D), Clinical Examples (CE), Learning Objectives (LO), University of Louisville's Neuroanatomy Reputation (UL-REP)] on a 5-point Likert scale related to learning neuroanatomy $(1=$ Not at All Helpful, 2= Slightly Helpful, 3= Moderately Helpful, 4= Very Helpful, and 5= Extremely Helpful) (Appendix B).

Data Analysis. Initially, descriptive statistics of neuroanxiety levels of all participants will be performed to explore the level of neuroanxiety of respondents. Subsequently, 
neuroanxiety levels will be categorized by gender and medical school class at our institution. Independent $t$-tests will be performed to investigate differences in neuroanxiety between gender and medical school class. If a difference in neuroanxiety between genders is found, a factorial ANOVA will be performed to further investigate the differences in gender and any previous neuroanatomy and/or neuroscience experience on neuroanxiety levels. An effect size, Cohen's $d$, will be calculated to determine the magnitude of the significant differences. A Cohen's $d$ of 0.2 will be considered a small effect size, 0.5 a medium effect size and $\geq 0.8$ a large effect size (Cohen, 1992). The significance level used for hypothesis testing will be held at $p<0.05$.

A multiple linear regression, using the enter method, will be performed on the upper-class medical student population (comprised of second-, third-, and fourth-year medical students) to investigate whether the predictor (independent) variables explain a significant amount of variability in neuroanxiety (dependent variable). The eight predictor variables to be investigated are: gender $(\mathrm{G})$, previous neuroanatomy and/or neuroscience experience (PNE), lecture (Lec), lab (L), 3D models (3D), clinical examples (CE), learning objectives (LO), and the University of Louisville's neuroanatomy reputation (UL-REP). The equation being investigated is:

$$
\text { Neuroanxiety }=b_{0}+b_{1 G}+b_{2 P N E}+b_{3 L E C}+b_{4 L}+b_{53 D}+b_{6 C E}+b_{7 L O}+b_{8 U L R E P}
$$

We are interested in which specific predictor variables most strongly predict neuroanxiety levels. First, Pearson correlations will be performed to assess for multicollinearity between the predictor variables. A high correlation between any two 
independent variables would suggest there is a strong relationship between those two predictors and a problem with multicollinearity. A further check of the variance inflation factor, greater than 5, in the regression output from SPSS ${ }^{\circledR}$ Version 26 would determine of the predictor variable should be removed from the model (Daoud, 2017). The fit of the regression model will be determined by both the F-statistic and the $R^{2}$. A significant Fstatistic with the greatest $R^{2}$, or proportion of variance explained, will determine the best fit for the model. If there are predictors that are found not to be statistically significant, those factors will be removed from the model and subsequent linear regression models will be explored to determine if a better fit model explains more of the variance in neuroanxiety. The significance level used for this analysis will be held at $p<0.05$.

\subsubsection{Results}

3.3.4.1 Predictors of Neuroanxiety. To determine which independent variables most strongly predict neuroanxiety, multiple linear regression analysis was conducted on the upper-class medical student population $(n=111)$ to evaluate the independent variables gender $(\mathrm{G})$, premedical neuroanatomy and/or neuroscience experience (PNE), lecture (Lec), lab (L), 3D models (3D), clinical examples (CE), learning objectives (LO), and the University of Louisville's neuroanatomy reputation (UL-REP). No strong Pearson's correlations were found between the independent variables suggesting multicollinearity. The correlation between clinical examples (CE) and 3D models (3D), $r=0.31$, was the highest correlation reported. Descriptive statistics of both the dependent variable (neuroanxiety) and predictor variables are presented in Table 3.6. A significant regression equation was found ( $F[5,105]=8.68, p<0.001)$ with an $R^{2}$ of 0.29 , suggesting $29 \%$ of the variance is predicted by the variables in the following regression equation. This was 
the best fit model; however, this is still a low proportion of the overall variance explained in neuroanxiety. The respondents predicted neuroanxiety is equal to $8.015+2.12 * \mathrm{G}-$ $1.76 * \mathrm{PNE}-1.74 * \mathrm{Lec}+1.58 * 3 \mathrm{D}-0.93 * \mathrm{CE}$. However, learning objectives $(\mathrm{LO})(p=$ $0.29)$, laboratory (L) ( $p=0.62)$, and the University of Louisville's neuroanatomy reputation (UL-REP) $(p=0.58)$ were not significant predictors of the model.

Neuroanxiety levels are highest in female medical students lacking in previous neuroscience experience with a dissatisfaction in lecture, and desire for more 3D models and clinical experiences in their medical education (Table 3.7).

3.5 Overall Discussion. The data show the presence of neuroanxiety at our institution: (1) upper-class medical students experienced greater neuroanxiety than first-year medical students; and (2) female medical students, even with previous neuroscience and/or neuroanatomy experience, exhibited greater neuroanxiety than male medical students without any previous experience. Our findings that female gender (Kam et al., 2013), lack of clinical case integration within the curriculum (Youssef, 2009; Abulaban et al., 2015; Javaid et al., 2018), lecture difficulties (Youssef, 2009; Abulaban et al., 2015; Shiels et al., 2017; Javaid et al., 2018), and a lack of 3D imaging (Javaid et al., 2018) predict neuroanxiety is consistent with previous studies on contributors of neurophobia. The novel finding from this study shows previous neuroscience experience predicts a decrease in neuroanxiety. Our data show learning objectives and laboratory instruction did not significantly predict neuroanxiety.

Our findings that female gender, lecture difficulties, lack of 3D models, and difficult clinical examinations predicted neuroanxiety is consistent with previous 
neurophobia studies (Youssef, 2009; Kam et al., 2013; Pakpoor et al., 2014; Abulaban et al., 2015; Anwar et al., 2015; Shiels et al., 2017; Javaid et al., 2018). First, our findings that female gender predicted neuroanxiety in medical students aligns with data from the Kam et al. (2013) study. Kam et al. (2013) also reported female gender predicted neurophobia in medical students, which is supported by other studies showing female medical students score higher on neurophobia surveys (Pakpoor et al., 2014; Anwar et al., 2015). Our results, similar to Kam et al. (2013), conflict with the Shiels et al. (2017) data showing no significant neurophobia gender differences in medical students. Another variable in the model that predicted neuroanxiety, lack of quality and effectiveness of lectures, is consistent with previous studies (Youssef, 2009; Abulaban et al., 2015; Javaid et al., 2018) suggesting didactic neuroanatomy lecture experiences contribute to neurophobia. The qualitative study in Chapter 2 of this dissertation highlighted lecture spacing, organization, and overestimation of student's base neuroanatomy knowledge during lectures (Table 2.4) as contributing to medical student neuroanxiety. The desire for more 3D models, a third predictor variable, is supported by the literature suggesting a lack of 3D models to aid in visualization as another potential contributor of neurophobia (Youssef, 2009; Abulaban et al., 2015; Javaid et al., 2018). This is further supported by previous studies reporting increased test scores with 3D models when compared to 2D instruction (Estevez et al., 2010; Kockro et al., 2015; Allen et al. 2016). Neuroanatomy clinical cases is another variable in our model that predicted neuroanxiety and is supported by the literature (Schon et al., 2002; Youssef, 2009; Abulaban et al., 2015; Javaid et al., 2018). Previous studies described both the difficulty of neurological cases (Schon et al., 2002) and the lack of clinical cases in the curriculum (Youssef, 2009; 
Abulaban et al., 2015; Javaid et al., 2018) as potential contributors of neurophobia. Our data in this study is consistent with student responses from the discussion group in Chapter 2 of this dissertation. Students report not enough clinical content in the curriculum (Table 2.2) and difficult neurological cases (Table 2.1) as contributing to neuroanxiety at our institution. Our findings support previous studies in the literature that female gender, lecture difficulty, lack of 3D models, and difficult neurological cases predict neuroanxiety.

Our investigation into the predictor variables of laboratory instruction, learning objectives, and upper-class neuroanatomy reputation has not previously been explored in the neurophobia literature to our knowledge. However, our data show these factors did not significantly predict neuroanxiety in our model. We anticipated laboratory instruction would predict neuroanxiety due to student frustration with the instruction and faculty inconsistencies in the neuroanatomy laboratory reported in the Chapter 2 study of this dissertation (Table 2.3). Concurrent lecture and cadaveric laboratory experiences in neuroanatomy have been described to benefit medical student academic success (Macchi et al., 2007; Rae et al., 2016), but to our knowledge has not yet been explored in the neurophobia literature. Learning objectives were another instructional strategy we explored in contributing to neuroanxiety. We hypothesized learning objectives would predict neuroanxiety from the literature and our preliminary findings in the Chapter 2 study. Students described uncertainty around what to study increased their general anxiety in Chapter 2 of this dissertation. This is consistent with the Uncertainty and Anticipation Model of Anxiety (UAMA) (Grupe \&Nitschke, 2013) showing uncertainty promotes maladaptive anxiety responses. However, our data showed unclear learning 
objectives did not significantly predict neuroanxiety at our institution. Lastly, neuroanatomy has been described in the literature as being a difficult subject (Schon et al., 2002; Youssef, 2009; Abulaban et al., 2015), but has not yet been explored as a predictor of neuroanxiety. We explored the possibility of upper-class medical students at our institution influencing first-year medical students opinions about neuroanatomy upon their arrival to medical school. Our data did not show neuroanatomy's reputation for being a difficult subject at our institution predicted neuroanxiety. In summary, these findings add to the existing literature by showing laboratory instruction, inadequate learning objectives, and neuroanatomy's course poor reputation did not significantly contribute to neuroanxiety at our institution. Taken together with the significant predictors of neuroanxiety (female gender, lacking premedical experience, lectures difficulties, lack of 3D models, and difficult clinical cases), these data informed the development of a learning intervention designed to reduce neuroanxiety. In Chapter 5 of this dissertation, the effect of this learning intervention on neuroanxiety will be explored.

Data from this study show the presence of neuroanxiety at our institution with two distinct populations exhibiting greater neuroanxiety: upper-class and female medical students. The finding that upper-class (second-, third-, and fourth-year) medical students had higher neuroanxiety scores than first-year medical students may give researchers insight regarding the timing of neuroanxiety development in medical students. Our results suggest that students do not enter medical school with neuroanxiety, but develop it over time due to negative experiences during their medical education. Our data confirm the Shiels et al. (2017) reports of a higher prevalence of self-reported neurophobia (26\%) in second-year medical students when compared to first year-medical students (19\%). 
However, these results conflict with the Kam et al. (2013) data showing low neuroanatomy knowledge of first-year medical students predicted greater neuroanxiety. Therefore, a future study may be needed to differentiate neuroanxiety levels between medical student classes, differentiating between first-, second-, third-, and fourth-year students, to determine when neuroanxiety is at its peak in medical education. Female students reported greater neuroanxiety scores than male students, as anticipated from a previous study on neurophobia and gender (Kam et al., 2013). However, the interaction effect of premedical neuroscience and/or neuroanatomy experience and gender on neuroanxiety has not previously been explored in the literature. Given our findings of both gender and premedical experience predicting neuroanxiety, we anticipated female medical students, even with premedical experience, would exhibit greater neuroanxiety. Although not statistically significant $(p=0.06)$, our novel data shows female medical students with premedical neuroscience and/or neuroanatomy experience reported higher neuroanxiety levels than their male classmates without neuroanatomy experience.

Data from this study supported initial validity and reliability evidence along with the development of this novel scale. Exploratory factor analysis found the NAS is a short, yet informative, scale to measure one construct, neuroanxiety, based on the framework of the GAD-7 (Spitzer et al., 2006). The internal consistency (Cronbach's $\alpha=0.91$ ), namely the inter-relatedness of the scale items, shows the extent to which all scale items are measuring the same construct. Scales measuring emotions towards neuroanatomy and beliefs towards neuroanatomy administered concurrently with the NAS found significant correlations $(p<0.0001)$ with neuroanxiety scores. Lower scores suggested more fearbased emotions towards neuroanatomy while higher scores reflect excitement, or 
positive, emotions towards neuroanatomy based on the 3-point scoring system in the Dictionary of Affect in Language (Whissell, 1989; Whissell, 2009). A negative correlation would be anticipated between high neuroanxiety scores and low emotions and beliefs towards neuroanatomy scores. Since a strong negative correlation between these scores was observed, this suggests the NAS score is reflecting both fear-based emotions and beliefs towards neuroanatomy. This provides convergent validity evidence the NAS score is a measure capturing the neuroanxiety construct. Alternatively, a scale designed to measure an alternative construct, with a documented negative correlation in the literature, should provide the same evidence to support discriminant validity. The alternative construct, educational self-efficacy, has been shown to be negatively correlated with educational anxiety in the literature (van Dinther et al., 2011; Barrows et al., 2013; Razavi et al., 2018). Higher neuroanatomy self-efficacy scores reflect more task-specific confidence in completing neuroanatomy tasks (scale development of the neuroanatomy self-efficacy scale (NSES) is described in Chapter 4 of this dissertation). Our findings that NAS scores are negatively correlated with NSES scores is consistent with persons with high educational anxiety exhibiting less educational self-efficacy. This provides discriminant validity evidence the NAS score is reflecting a separate and distinct construct from the neuroanatomy self-efficacy score.

There were a few limitations to both parts of our study; sample size was major limitation of the regression analysis and lacking a score to obtain criterion validity was one major limitation of the scale development. The sample size $(n=111)$ may have been smaller than the ideal sample size for an 8-variable predictor regression model. Tabachnick and Fidell (2007, p. 123) advise 50 observations per predictor variable in a 
multiple regression to derive a reliable error term for the model. Alternatively, Altman (1991, p. 349), suggests a minimum of 10 observations per predictor variable is the minimum number of observations to derive an error term for the model. Our sample size of 111 was larger than the minimum required 80 observations; however, a larger sample size may have detected a bigger effect, relative to the error in the data, and a larger proportion of the explained variance in the model. Our results need to be taken cautiously, as the best fit regression model showed an overall low proportion of explained variance (29\%). Another limitation of our study was the inability to screen our students for anxiety disorders, due to lack of mental health professionals on the research team. This limited our ability to obtain criterion validity, by correlating the neuroanxiety scores with a published valid and reliable scale, the GAD-7 (Spitzer et al., 2006). To account for this limitation, a number of scales (Neuroanatomy Emotions Scale, Neuroanatomy Beliefs Scale, Neuroanatomy Self-Efficacy Scale) were created to explore the convergent validity (Neuroanatomy Emotions \& Beliefs Scales) and the divergent validity (Neuroanatomy Self-Efficacy Scale) of the novel scale. Additionally, screening our respondents for an anxiety disorder would allow the researchers to control for differences in pre-course anxiety levels when exploring medical student neuroanxiety scores.

In summary, female gender, lecture difficulties, difficult clinical cases and lack of 3D models to learn neuroanatomy predict neuroanxiety at our institution. Learning objectives, laboratory instruction, and neuroanatomy's reputation did not predict neuroanxiety. This study informed the development of a learning intervention, of which the effects on neuroanxiety will be investigated in Chapter 5 using the novel scale developed in this study. These data also provide information to medical educators that 
focusing resources on improving lecture, clinical case integration, and providing access to 3D technology may potentially reduce neuroanxiety in medical students. Differences in first-year compared to upper-class medical students need to be studied further to isolate when neuroanxiety is at its peak in medical school. Devoting resources focusing more heavily on the medical student population exhibiting the most neuroanxiety may have the most overall educational impact. Gender specific differences in neuroanxiety, particularly with premedical neuroscience and/or neuroanatomy experiences, may inform premedical neuroscience and neuroanatomy youth educational programs designed to target girls. This study showed female medical students with previous neuroscience and/or neuroanatomy experiences exhibit less neuroanxiety than their female classmates with no premedical experiences. More data in a future study is needed to further investigate this relationship. 


\section{TABLE 3.1}

\begin{tabular}{|l|l|}
\hline \multicolumn{2}{|c|}{ Student Responses Supporting DSM-5 GAD Symptoms } \\
\hline $\begin{array}{l}\text { Symptomatic } \\
\text { Diagnostic } \\
\text { Criteria }\end{array}$ & Supporting Key Quote(s) \\
\hline $\begin{array}{l}\text { Restless or } \\
\text { Feeling on } \\
\text { Edge ( } n=7)\end{array}$ & $\begin{array}{l}\text { Student: I've never even like worked with brains before in my life so it's just really } \\
\text { overwhelming } \\
\text { Student: ...I was so worried about, oh my gosh, I have to memorize all these pictures } \\
\text { Student: really intricate cases were...extremely overwhelming to hear that the lesion } \\
\text { could have been in like thirty different places most of which we never heard of before... }\end{array}$ \\
\hline $\begin{array}{l}\text { Fatigued } \\
(n=3)\end{array}$ & $\begin{array}{l}\text { Student: ...having four straight hours of neuroanatomy lecture in the morning is } \\
\text { exhausting and disheartening }\end{array}$ \\
\hline $\begin{array}{l}\text { Difficulty } \\
\text { concentrating } \\
(n=8)\end{array}$ & $\begin{array}{l}\text { Student: I liked having the break between them ...I think like it gave my brain a little } \\
\text { rest.... } \\
\text { Student: I became so incredibly overwhelmed that the rest of the day- I think we had } \\
\text { two other lectures that day- and the next day there were three- I just lost it } \\
\text { Student: So where is this lesion? It's like I can't remember forty minutes ago I don't } \\
\text { even remember my name right now... }\end{array}$ \\
\hline $\begin{array}{l}\text { Irritability } \\
\text { which } \\
\text { impaired } \\
\text { occupational } \\
\text { functioning } \\
\text { (n=12) }\end{array}$ & $\begin{array}{l}\text { Student: Well, I came in kind of really excited about neuroanatomy because I like the } \\
\text { brain, and that is sort of what I've always liked, but I was quickly crushed, or defeated, } \\
\text { or like quickly starting hating this }\end{array}$ \\
$\begin{array}{l}\text { Student: I was really intimidated and overwhelmed and I thought, oh, this is horrible } \\
\text { Student: It would be helpful...if (professors didn 't) just keep telling you how much you } \\
\text { don't know } \\
\text { Student:... it's a lot harder to relearn information a different way after you've been } \\
\text { given the information incorrectly... I just got mad and then I didn't learn anything from } \\
\text { that because I was just angry }\end{array}$ \\
\hline
\end{tabular}


TABLE 3.1 Student Responses Supporting DSM-5 GAD Symptoms. Supporting key student quotes, and total number of responses, are provided for each of the four diagnostic criteria for anxiety. 


\section{TABLE 3.2}

\begin{tabular}{|c|c|}
\hline \multicolumn{2}{|r|}{ Student Responses Supporting Three-Dimensional Model of Neuroanxiety } \\
\hline Factors & Supporting Key Quote(s) \\
\hline $\begin{array}{l}\text { Physiological } \\
(n=7)\end{array}$ & $\begin{array}{l}\text { Student: ...I was so worried about, oh my gosh...I couldn't sit through class } \\
\text { Student: ... having four straight hours of neuroanatomy lecture in the morning is } \\
\text { exhausting and disheartening } \\
\text { Student: ... I just got mad and then I didn't learn anything from that because I was just } \\
\text { angry (student observably speaking loudly with increased respiration depth and rate) }\end{array}$ \\
\hline $\begin{array}{l}\text { Cognitive } \\
(n=3)\end{array}$ & $\begin{array}{l}\text { Student: So where is this lesion? It's like I can't remember forty minutes ago I don't } \\
\text { even remember my name right now... } \\
\text { Student: I was really intimidated and overwhelmed and I thought, oh, this is horrible } \\
\text { Student: ...I was quickly crushed, or defeated, or like quickly starting hating this }\end{array}$ \\
\hline $\begin{array}{l}\text { Behavioral } \\
(n=3)\end{array}$ & $\begin{array}{l}\text { Student: I would get rid of the first neuro lecture (of the day) because that set the tone } \\
\text { for me and I became so incredibly overwhelmed the rest of the day... I just lost it (and } \\
\text { went home) } \\
\text { Student: I didn't really like spend enough time because I was so frustrated by the } \\
\text { quiz... }\end{array}$ \\
\hline
\end{tabular}


TABLE 3.2 Student Responses Supporting Three-Dimensional Model of Neuroanxiety. Supporting key student quotes, and total number of responses, are provided for each of the three dimensions of anxiety- physiological, cognitive, and behavioral. 
TABLE 3.3

\begin{tabular}{|c|c|c|c|c|c|c|c|c|c|c|}
\hline Class & $N$ & $\begin{array}{c}\mathbf{N M} \\
(n)\end{array}$ & & $\begin{array}{l}\mathbf{G} \\
\mathbf{P} \\
\mathbf{A}\end{array}$ & $\begin{array}{c}\text { BCPM } \\
\text { GPA }\end{array}$ & MCAT & GeI & der & Age & $\overline{\text { UIM }}$ \\
\hline 2015 & 154 & 3 & & 3.66 & 3.58 & $*$ & $58 \%$ & $42 \%$ & 24 & $14 \%$ \\
\hline 2016 & 156 & 2 & & 3.70 & 3.63 & 507 & $52 \%$ & $48 \%$ & 24 & $12 \%$ \\
\hline 2017 & 155 & 2 & & 3.73 & 3.65 & 507 & $54 \%$ & $46 \%$ & 23 & $6 \%$ \\
\hline 2018 & 160 & 8 & & 3.65 & 3.55 & 508 & $57 \%$ & $43 \%$ & 23 & $11 \%$ \\
\hline 2019 & 162 & 8 & & 3.68 & 3.59 & 508 & $54 \%$ & $46 \%$ & 22 & $12 \%$ \\
\hline Total: & 787 & 23 & Avg: & 3.68 & 3.60 & 507.5 & $55 \%$ & $45 \%$ & 23.2 & $11 \%$ \\
\hline
\end{tabular}




\section{TABLE 3.3 Study Population: Class profile demographics for ULSOM entering}

classes 2015 to 2019. $N=$ number of matriculated students; $N M=$ Number of neurobiology or neuroscience majors; $G P A=$ overall grade point average ( 4.0 scale); $B C P M G P A=$ overall Biology, Chemistry, Physics and Mathematics grade point average (4.0 scale); $M C A T=$ Medical College Admissions Test scores (* Entering class 2015 scores were graded on the old system and not included in the average. Entering Class 2015 MCAT scoring= Verbal: 9.68, Physical Science: 9.53; Biological Science 10.05); Gender $=$ binary gender listed as male $(\mathrm{M})$ and female $(\mathrm{F}) ;$ Age $=$ average age of entering class; $U I M=$ underrepresented in medicine reflecting racial diversity of the entering class. 
TABLE 3.4

\begin{tabular}{|c|c|c|c|c|c|}
\hline \multirow{2}{*}{$\begin{array}{c}\text { All Medical } \\
\text { Student } \\
\text { Matriculants }\end{array}$} & \multirow[t]{2}{*}{$\overline{\text { GPA }^{1}}$} & \multirow{2}{*}{$\begin{array}{c}\text { BCPM } \\
\text { GPA }^{1}\end{array}$} & \multirow[t]{2}{*}{ MCAT $^{1}$} & \multicolumn{2}{|c|}{ Gender $^{2}$} \\
\hline & & & & Male & Female \\
\hline 2016 & 3.70 & 3.64 & 508.7 & $50.2 \%$ & $49.8 \%$ \\
\hline 2017 & 3.71 & 3.64 & 510.4 & $49.3 \%$ & $50.7 \%$ \\
\hline 2018 & 3.72 & 3.65 & 511.2 & $48.4 \%$ & $51.6 \%$ \\
\hline 2019 & 3.73 & 3.66 & 511.5 & $47.6 \%$ & $52.4 \%$ \\
\hline
\end{tabular}




\section{TABLE 3.4 MCAT Scores, GPAs, and Gender Composite of Matriculants to US}

Medical Schools, 2016 through 2019. Data from the Association of American Medical Colleges (AAMC) showing the characteristics of total incoming matriculants to US Medical School for the same timeframe as this study. $G P A=$ overall grade point average (4.0 scale); $B C P M G P A=$ overall Biology, Chemistry, Physics and Mathematics grade point average (4.0 scale); $M C A T=$ Medical College Admissions Test scores; Gender= binary gender listed as male and female.

${ }^{1}$ Association of American Medical Colleges. 2020. MCAT Scores and GPAs for Applicants and Matriculants to U.S. Medical Schools, 2016-2017 through 2020-2021. [https://www.aamc.org/data-reports/students-residents/interactive-data/2020-factsapplicants-and-matriculants-data]. Accessed [28 February 2020]

${ }^{2}$ Association of American Medical Colleges. 2020. Applicants, First-Time Applicants, Acceptees, and Matriculants to U.S. Medical Schools by Sex, 2011-2012 through 20202021. [https://www.aamc.org/data-reports/students-residents/interactive-data/2020-factsapplicants-and-matriculants-data]. Accessed [28 February 2020] 


\section{TABLE 3.5}

\begin{tabular}{|l|l|l|}
\hline Item & $\begin{array}{l}\text { Factor } \\
\text { Matrix }\end{array}$ & $\boldsymbol{h}^{\mathbf{2}}$ \\
\hline $\begin{array}{l}\text { 2 Not being able to stop or control thinking neuroanatomy is too } \\
\text { difficult }\end{array}$ & 0.85 & 0.72 \\
\hline $\mathbf{3}$ Worrying too much and not being able to focus on neuroanatomy & 0.84 & 0.70 \\
\hline $\begin{array}{l}\text { 7 Feeling afraid that I either won't pass neuroanatomy or properly } \\
\text { diagnose a neurology patient }\end{array}$ & 0.79 & 0.62 \\
\hline 6 Becoming easily annoyed or irritable & 0.78 & 0.61 \\
\hline $\mathbf{1}$ Feeling nervous, anxious or on edge & 0.77 & 0.59 \\
\hline $\begin{array}{l}\text { 4 Trouble completing lesion isolation case scenario or clinical } \\
\text { problems }\end{array}$ & 0.69 & 0.48 \\
\hline 5 Being so restless that it's hard to sit still & 0.62 & 0.39 \\
\hline
\end{tabular}




\section{TABLE 3.5 Factor Matrix and Extraction Communalities (Principal Axis}

Factoring). The factor matrix demonstrates a high correlation of all items loading onto

one factor; $h^{2}=$ communalities, or the proportion of each item's variance explained by the factor; Oblimin rotation was used. 


\section{FIGURE 3.1}

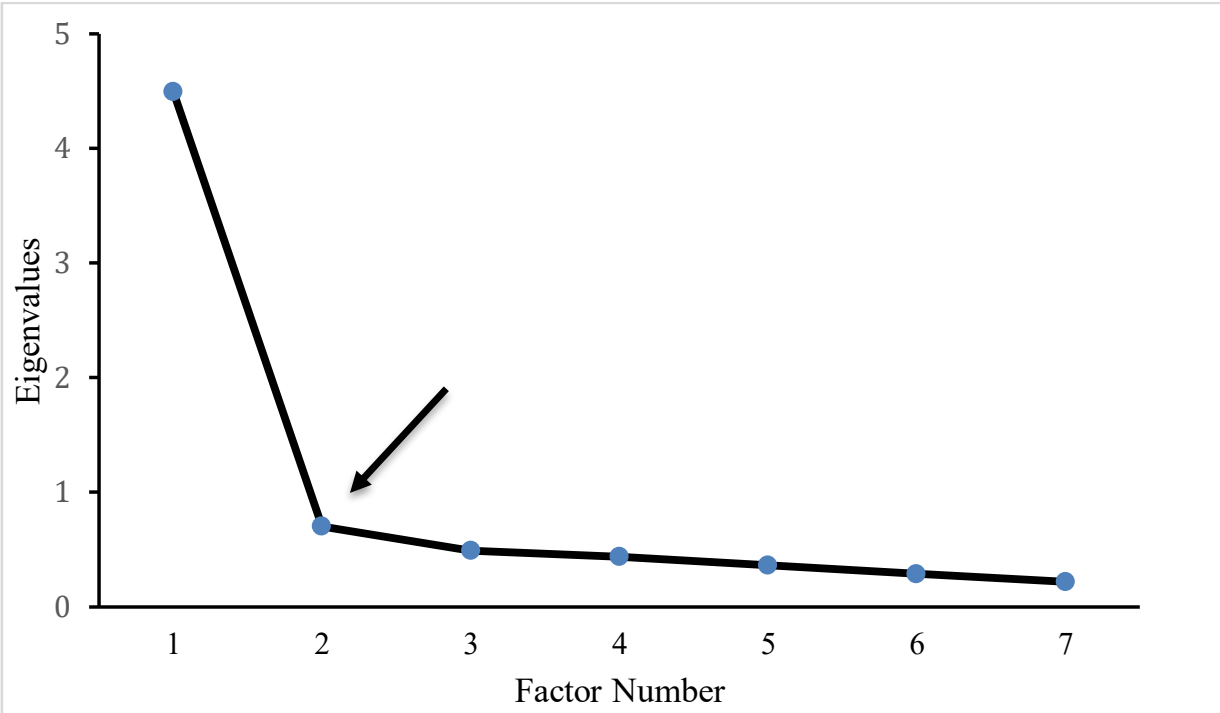


FIGURE 3.1 Scree Plot. The elbow of the scree plot (arrow) shows a one factor solution for this scale (with only one eigenvalue $>1$ ). The elbow of the scree plot is the sharp bend where the eigenvalues begin to level off. The factors to the left of the sharp bend, or elbow, are considered significant and should be retained. 


\section{FIGURE 3.2}

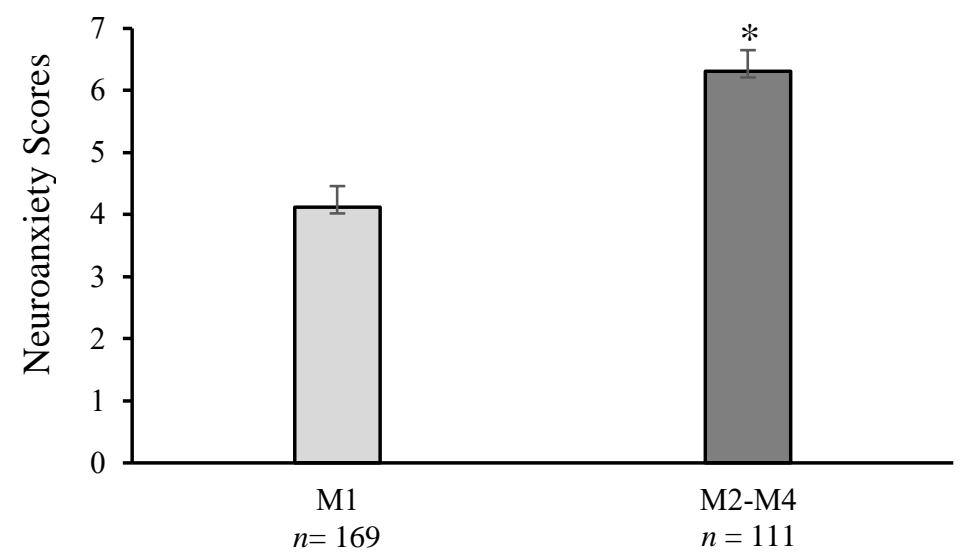

Medical Student Class 
FIGURE 3.2 Neuroanxiety scores (reported values are mean \pm S.E.) based on medical student class. Upper-class medical students (M2-M4) reported overall higher levels of neuroanxiety than first-year medical students (M1). The asterisk (*) indicates a significant difference $(p<0.001)$ in neuroanxiety levels of male students compared to female students. A medium effect size was observed (Cohen's $d=0.49$ ) indicating moderate practical significance. 


\section{FIGURE 3.3}

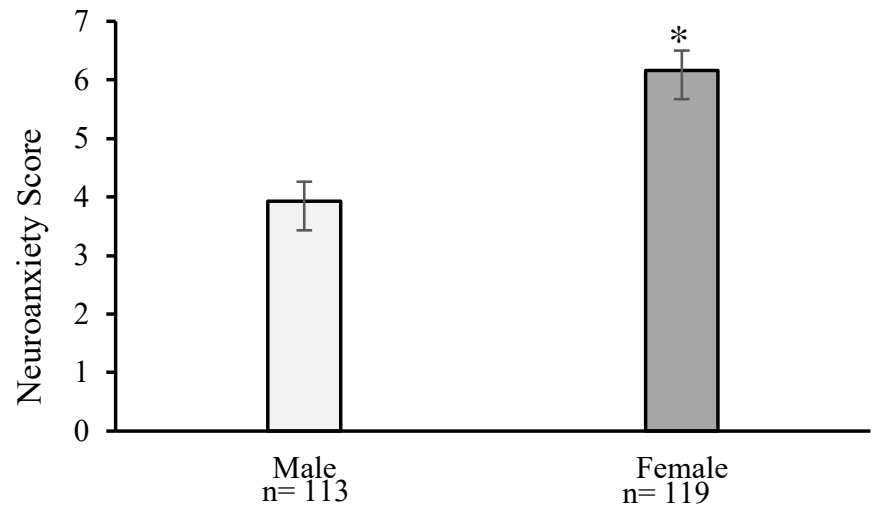

Binary Gender 
FIGURE 3.3 Neuroanxiety scores (reported values are mean \pm S.E.) based on

binary gender. Female medical students reported nearly double the neuroanxiety scores than male medical students. The asterisk $(*)$ indicates a significant difference $(p<0.001)$ in neuroanxiety levels of male students compared to female students. A medium effect size was observed (Cohen's $d=0.52)$ indicating moderate practical significance. 


\section{FIGURE 3.4}
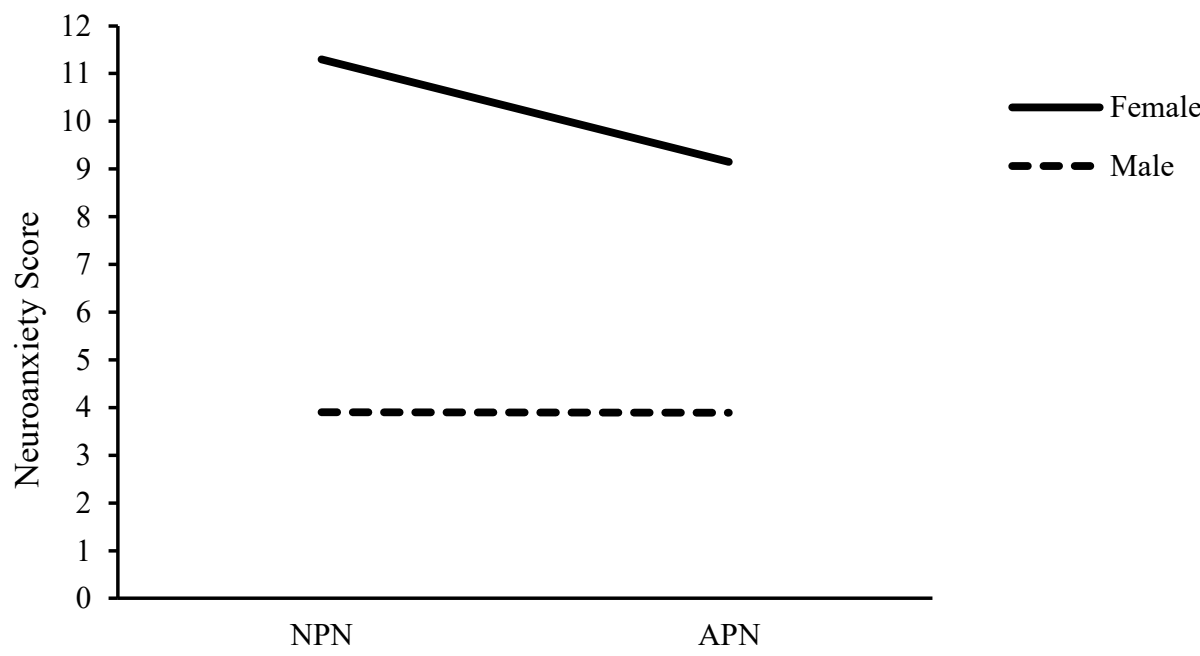

- - Male

Premedical Neuroscience and/or Neuroanatomy Experience 
FIGURE 3.4 Estimated marginal means of neuroanxiety score. The interaction effect between gender and previous neuroscience experience on neuroanxiety shows no significant difference $(p=.06)$. The data line is trending towards female students without any premedical neuroanatomy (NPN) and/or neuroscience experience show greater neuroanxiety levels than their female classmates with previous neuroanatomy and/or neuroscience experience (APN). 
TABLE 3.6

\begin{tabular}{|l|c|c|c|}
\hline \multicolumn{1}{|c|}{ Variable } & Mean & $\begin{array}{c}\text { Standard } \\
\text { Deviation }\end{array}$ & $\boldsymbol{n}$ \\
\hline DV: Neuroanxiety Score & 6.31 & 4.63 & 111 \\
\hline IV1: Lecture & 2.85 & 0.95 & 111 \\
\hline IV2: Laboratory & 3.06 & 1.19 & 111 \\
\hline IV3: 3D Models & 2.55 & 1.02 & 111 \\
\hline IV4: Clinical Examples & 3.42 & 1.02 & 111 \\
\hline IV5: Learning Objectives & 2.68 & 1.09 & 111 \\
\hline IV6: UL Neuroanatomy Reputation & 2.75 & 1.12 & 111 \\
\hline $\begin{array}{l}\text { IV7: Any premedical neuroanatomy and/or } \\
\text { neuroscience experience }\end{array}$ & 0.49 & 0.50 & 111 \\
\hline IV8: Gender & 1.53 & 0.50 & 111 \\
\hline
\end{tabular}


TABLE 3.6. Descriptive Statistics of Variables. Scores of the dependent variable (DV) and each one of the independent variables (IV1-IV6) are reported as mean values \pm Standard Deviation. Upper-class (second-, third- and fourth year) medical student data were used for the regression analysis. 
TABLE 3.7

\begin{tabular}{|c|c|c|c|c|c|}
\hline \multicolumn{4}{|c|}{ Unstandardized Coefficients } & \multirow[b]{2}{*}{$t$} & \multirow[b]{2}{*}{ Sig. } \\
\hline & $b$ & $\begin{array}{r}\text { Std. } \\
\text { Error }\end{array}$ & $\beta$ & & \\
\hline (Constant) & 8.015 & 2.102 & & 3.813 & $<<.001$ \\
\hline Gender $(\mathrm{G})$ & 2.120 & .769 & .230 & 2.755 & .007 \\
\hline Premedical Neuro (PNE) & -1.756 & .766 & -.190 & -2.292 & .024 \\
\hline 3D Models (3D) & 1.581 & .420 & .347 & 3.764 & $<.001$ \\
\hline Lecture Instruction (Lec) & -1.736 & .454 & -.355 & -3.828 & $<.001$ \\
\hline Clinical Examples (CE) & -.931 & .438 & -.206 & -2.127 & .036 \\
\hline
\end{tabular}


TABLE 3.7. Regression Model. Predicting neuroanxiety from gender (G), premedical neuroscience experience (PNE), 3D models (3D), lectures (Lec), and clinical examples (CE) in the curriculum. Fit for model $R^{2}=.292$, Adjusted $R^{2}=.259, F(5,105)=8.68, p<$ .001 . 


\section{CHAPTER 4}

\section{EFFECTS OF PREMEDICAL NEUROANATOMY EXPERIENCES AND GENDER ON NEUROANATOMY SELF-EFFICACY IN AN INTEGRATED MEDICAL CURRICULUM}

4.1 Introduction. Self-efficacy and premedical educational experiences are two related factors that influence student success in medical school (Mavis, 2001; Foreseter et al., 2002; Peterson and Tucker, 2005; Burgoon et al., 2012; Young et al., 2012; Bierer et al., 2015; Kondrashov et al., 2017; Hayat et al., 2020). Self-efficacy is defined as, “one's own perception of their ability to succeed at a task" (Bandura, 1977, p. 193) and the judgements one makes about their own abilities to complete tasks reflects their level of self-efficacy (Schunk, 1991). Self-efficacy is considered the critical agentic mechanism (Bandura, 1997), meaning one's belief in their ability to make things happen, and occupies a pivotal role in Social Cognitive Theory (SCT) (Bandura, 2001). In an academic setting, students with high self-efficacy believe they are capable of succeeding on exams and have an influence on producing a desired outcome, such as high grades. Self-efficacy in medical education research has been found not only to be related to academic performance, but also to persistence, motivation, and may even protect against medical student burnout. Educational experiences before entering medical school may provide a student additional benefit by increasing their self-efficacy through a student's 
perception of their own abilities. Premedical experiences, specifically related to cadaveric dissection, appear to provide the most benefit to first-year medical students learning gross anatomy in academic performance (Foreseter et al., 2002; Peterson \& Tucker, 2005; Kondrashov et al., 2017). However, an investigation into varying premedical neuroanatomy experiences and neuroanatomy specific self-efficacy has not yet been explored. This study aims to fill this gap in the literature.

Academic self-efficacy improves overall performance in medical school. Medical students with increased self-efficacy performed better in the first year of medical school in gross anatomy (Burgoon et al., 2012) and on first-year final examinations (StegersJager et al., 2012; Hayat et al., 2020). Medical students with higher self-efficacy scored higher on clinical performance examinations (Mavis, 2001) and self-efficacy predicted a medical student's clinical performance (Opacic, 2003). After graduation, medical residents with higher self-efficacy demonstrated improved patient care experiences (Young et al., 2012). However, research has shown there is a complex relationship between academic self-efficacy and other aspects of learning that impact medical student performance. Self-efficacy may influence key constructs of medical student motivation and persistence leading to medical student success. Premedical experiences may also, in turn, influence academic self-efficacy. A review of the relationship between academic self-efficacy and other aspect of learning, namely academic motivation, persistence, and previous educational experiences, and medical student success from the medical educational literature will be explored.

The relationship with self-efficacy and motivation, specifically intrinsic motivation, in medical students (Roohi et al., 2013) has been explored in the literature. 
Intrinsic motivation is one's motivation to engage in an activity for its own sake (Pintrich \& Schunk, 2002) and students pursuing medicine for their own personal reasons, such as helping others or improving community health, would demonstrate greater intrinsic motivation. Medical students who show high intrinsic motivation exhibit high selfefficacy levels with increased academic performance (Roohi et al., 2013). However, this relationship between self-efficacy, intrinsic motivation and academic success may be complex. Wu et al. (2020) found self-efficacy itself did not predict academic performance. Instead, it was self-efficacy's influence on intrinsic motivation that led to improved academic performance (Wu et al., 2020). Self-efficacy's role in indirectly influencing academic performance by intrinsic motivation needs further study to be elucidated.

Self-efficacy is related to medical student persistence through a construct called grit. Grit is defined as "perseverance and passion for long-term goals" (Duckworth et al., 2007). In a recent study, self-efficacy was found to be a mediator of grit in university students (Alhadabi \& Karpinski, 2020). Students with higher levels of grit, and more selfefficacy, show greater persistence and consistency with overcoming obstacles on their way to reaching their career goals (Alhadabi \& Karpinski, 2020). Medical students with more grit performed better in gross anatomy (Fillmore \& Helfenbein, 2015), on course examinations (Miller-Matero et al., 2018; Alzerwi, 2020), national licensing examinations (Ursua et al., 2021), were more likely to graduate within four years (MillerMatero et al., 2018; Alzerwi, 2020), and exhibited less burnout syndrome (Jumat et al., 2020). Self-efficacy is an important factor in many aspects of medical student success and performance. 
Another predictor of medical student success of interest to this study is premedical experience. Premedical experiences, specifically with a cadaveric dissection laboratory component, are positively correlated with medical school grades and GPA in medical gross anatomy courses. Forester et al. (2002) found students with premedical gross anatomy with prosected cadaveric specimens showed significantly higher anatomy grades than students with premedical anatomy experience lacking cadaveric specimens. Peterson and Tucker (2005) similarly found premedical anatomy experience with laboratory dissection highly correlates with GPA and moderately correlates with gross anatomy grades in medical school. Kondrashov et al. (2017) studied varying premedical anatomy course experiences and perceived benefit for student success in medical gross anatomy. Students with three or more undergraduate courses, and/or specifically one with cadaveric specimens, provided the greatest perceived benefit for medical school gross anatomy success (Kondrashov et al., 2017). This perceived benefit for success may contribute to a student's own perception of their ability to perform a task- which reflects the construct of self-efficacy.

Study Purpose. A literature search found no studies to-date, or psychometrically validated scales, measuring neuroanatomy self-efficacy. This present study will fill this gap in the literature. The purpose of this study is to provide a theoretical framework for neuroanatomy self-efficacy, develop a novel instrument to measure it, and provide data for initial validity and reliability evidence for the instrument. Additionally, the influence of premedical neuroanatomy and/or neuroscience experience on has not yet been explored in the literature. A second purpose of this study is to explore the effect of 
various premedical neuroscience and/or neuroanatomy experiences- especially related to premedical cadaveric dissection- on neuroanatomy self-efficacy. The neuroanatomy selfefficacy scale developed in this study will allow medical education researchers the ability to measure neuroanatomy self-efficacy in medical students and assess the effects of curriculum changes or educational interventions on neuroanatomy self-efficacy. In Chapter 5 of this dissertation, this neuroanatomy self-efficacy scale will be used to assess a neuroanatomy learning intervention designed to improve medical student neuroanatomy self-efficacy.

This study unfolds with an exploration of self-efficacy which is based in Social Cognitive Theory. Subsequently, conceptualization of neuroanatomy self-efficacy and the sources of neuroanatomy self-efficacy will be described prior to developing a neuroanatomy self-efficacy scale and assessing premedical neuroanatomy and/or neuroscience experiences on medical student neuroanatomy self-efficacy.

\subsection{Background.}

Social Cognitive Theory and Self-Efficacy. Social Cognitive Theory (SCT)

hypothesizes the role one's personal agency plays in an environment (Bandura 2001).

Personal agency is the ability to intentionally make things happen by one's actions (Bandura, 2001). Bandura (2001) describes four core features of personal agency: intentionality, forethought, self-reactiveness and self-reflectiveness. People with strong personal agency can adequately assess their abilities (self-reflectiveness), can anticipate future challenges (forethought) while taking action and making course corrections along the way (self-reactiveness) to produce a desired outcome (intentionality). Self-efficacy is 
considered the critical agentic mechanism (Bandura et al., 1996) and occupies a pivotal role in SCT (Bandura, 2001). One's self-efficacy beliefs influence the level of challenge one undertakes, their effort, and perseverance when obstacles arise. Bandura (2001) adds that self-efficacy can determine whether failures are motivating or demoralizing through regulation of self-enhancing or self-defeating thoughts. In an educational environment, students with high self-efficacy can assess their own strengths and weaknesses (selfreflectiveness), can anticipate challenges on future examinations (forethought) while focusing on strengthening their weaknesses (self-reactiveness) to succeed academically (intentionality). The sources of self-efficacy, therefore, are critical in determining one's personal agency in an educational environment. Bandura (1977) outlined four sources of self-efficacy: Performance, Vicarious Experience, Verbal Persuasion and Emotional Arousal. Below is a description of how the sources of self-efficacy, within the context of SCT, could be applied to an educational environment (Bandura, 1977, 1996, 2001, 2012), for example medical school.

Performance mastery creates the strongest level of self-efficacy (Bandura, 2001). Medical students successfully completing examinations, performing cadaver dissections, and solving case-studies would encompass performance mastery. In the context of SCT, medical students need the forethought to anticipate what questions might be asked on examinations or problems that might arise during dissection, the self-reflectiveness to assess their weakness and take action to improve them (self-reactiveness). Their level of academic success is the result of their intentionality in these efforts. These successful experiences would successively improve their self-efficacy as they progress through medical school. 
Vicarious experience creates self-efficacy through modeling (Bandura, 2001). Firstyear medical students will encounter problems for which they have no prior experience. Innovative thought processes are required for solving these novel problems. Modeling can be achieved in the classroom by an instructor or in a peer-to-peer learning environment. An instructor may explain their step-by-step thought process in diagnosing a patient, for example, and provide students with common missteps. SCT posits medical students could use an instructor's forethought to anticipate these common mistakes. In a later work, Bandura (2005) explains modeling has been misconstrued as merely imitation thereby limiting creative thought. However, students observing an instructor's thought process modeling generally take pieces of the instructor's thought process and combine them with their own independent thoughts to create their own unique process. In this way, medical students use SCT by reflecting on their own thought processes (selfreflectiveness), taking actions to create new thoughts (self-reactiveness) in order to create the intended outcome (intentionality) of patient diagnosis.

Verbal persuasion is one of the least effective ways to promote self-efficacy (Bandura, 2001). Within the context of SCT, educational verbal persuasion may be most related to one's self-reflectiveness. For example, an instructor provides a verbal persuasion that accurately describes difficult material, This is a high-level problem, but I know you can handle it. The student may find the problem difficult, self-reflect on their own abilities and use this verbal persuasion as an inspiration to persist through the difficult task. The result of successful completion in this scenario may improve a student's self-efficacy. However, student self-beliefs about their own abilities may impair their self-efficacy if externally received verbal persuasion reflects a discord in a student's 
own self-reflection. For example, if an instructor provides a persuasion, such as "this is simple" but a student feels the problem is quite difficult, the verbal persuasion does not match the student's beliefs of their own abilities. The student may perceive their own level of self-efficacy as low if it they are having difficulty completing the problem because they do not feel they have the skills to succeed. A student's internal dialogue may affect their emotional arousal level, as described below. Verbal persuasion is one of the least effective ways to improve self-efficacy and should be used sparingly in a challenging educational environment like medical school.

Emotional arousal can improve, or limit, self-efficacy (Bandura et al., 1996) depending on how these emotions are interpreted. In this way, SCT's self-reflection and one's resulting actions (self-reactiveness) have direct connections to the assessments one makes about their own emotions. A medical student can feel emotional arousal (i.e., increased heart rate, breathing rate, sweating, etc...) and interpret this feeling as excitement which inspires a student to keep studying and learning through intentional action. Alternatively, another medical student may feel the same emotional arousal (i.e., increased heart rate, breathing rate, sweating...) and perceive this emotion as anxiety which demotivates study and preparation. It is in this way that SCT suggests anxiety and self-efficacy are related to each other. One's own self-reflection of their emotional arousal influences their anticipation of future challenges (forethought), actions (selfreactiveness), and the outcomes of those actions (intentionality).

Neuroanatomy self-efficacy defined. The proposed operational definition of the latent construct neuroanatomy self-efficacy is based on Bandura's original definition of self- 
efficacy, "one's own perception of their ability to succeed at a task" (Bandura, 1977, p. 193). In this study, neuroanatomy self-efficacy will be defined as: One's perceived ability to successfully complete a neuroanatomical or clinical neurological task.

Dimensions of neuroanatomy self-efficacy. Neuroanatomy self-efficacy is proposed to be a multidimensional construct, based on Bandura's $(1977,1986)$ self-efficacy theory, with three dimensions: magnitude, generality, and strength. Magnitude is the amount of perceived neuroanatomical knowledge and/or skill that is required to perform a certain task. These skills are ranked in ascending order of difficulty level. In this case, the task could be a basic question about the location of a nucleus on a cross-sectional image, or a more advanced clinical case scenarios, lesion problems or neurological exam skills. Generality is the amount of transferability of neuroanatomical knowledge and/or skills from a specific to a more general situation. For example, knowing where the neuroanatomical tracts decussate (cross) in the spinal cord is foundational neuroanatomy knowledge. The quick retrieval of this information is a transferable skill to complete a clinical lesion problem on a written examination, and even further applicable to isolating a lesion in a patient when performing a neurological examination. A grasp of the foundational neuroanatomical knowledge will allow the student to transfer this information in many different clinical situations. Strength is the level of perseverance one demonstrates when performing neuroanatomical tasks and/or clinical skills. For example, a student with weak neuroanatomy self-efficacy would easily give up after failing to isolate the lesion on a clinical case scenario question. A student with strong neuroanatomy self-efficacy would persevere through the tough clinical case scenario 
question to isolate the lesion, despite multiple failures. The proposed instrument to measure neuroanatomy self-efficacy will encompass the three dimensions of Perceived Skill (Magnitude), Transferability (Generality), and Perseverance (Strength).

Sources of neuroanatomy self-efficacy. Understanding how the sources of self-efficacy are applied to neuroanatomy self-efficacy are essential to create instrument items to measure that construct. Below is a description of how each of the four sources of selfefficacy- Performance, Vicarious Experience, Verbal Persuasion and Emotional Arousalapply to neuroanatomy self-efficacy (Bandura, 1977, 1986, 2001, 2012).

Performance mastery creates the strongest level of self-efficacy (Bandura, 1977). Students successfully completing neuroanatomy questions in increasing level of difficulty. First order (identify) questions should be asked first before progressing to second (function) and third (clinical) order questions. Initially using "soft-feedback" terms such as "not quite" or "on the right track" instead of "correct" and "wrong" when completing first-order questions will not dissuade the student during the initial mastery experience. As the questions progress into the second and third order type questions, guiding students with explanations to the correct answer is an important part of creating neuroanatomy self-efficacy. Intentionally increasing the level of difficulty, responding with soft feedback and explanations is intended to improve neuroanatomy self-efficacy through performance mastery.

It has been shown that vicarious experience, especially when modeled by someone who is similar to the student in anxiety and confidence level, creates self-efficacy through modeling (Bandura, 1977). A new thought process, especially for second and third order 
neuroanatomy questions with a high difficulty level, might be required to solve a problem. Modeling can be achieved in the classroom or through a video explanation. This instructor explains how they were "initially confused about this" or in areas of common misconceptions as "I thought it was this at first...but I was wrong in my thought process". Modeling someone who is unsure of how to answer the question is important in high difficulty level neuroanatomy problems. Not only modeling how to do the problem, but also modeling someone who is possibly feeling how they are currently feeling who overcame those feelings and succeeded anyway.

Verbal persuasion should be used minimally, as this is one of the least effective ways to promote self-efficacy. Verbal persuasion can fail if over utilized or used nonintentionally. If one provides persuasion, such as, "this is simple" and the problem they are about to attack is quite difficult, the verbal persuasion may fail. The student may perceive their own level of self-efficacy as low if it takes a long time to complete the problem because they do not feel they have the skills to succeed. If the verbal persuasion provided is a more accurate representation of the level of the material, "this is a highlevel problem, but I know you can handle it"- the result of successful completion of that scenario may improve student self-efficacy. It took them a long time to complete the problem, but they were correctly informed this was a high-level problem, and now they feel they have the skills to attack other high-level problems. This is how verbal persuasion can be used minimally, and hopefully effectively, to increase neuroanatomy self-efficacy.

Perception of high emotional arousal level is important on determining self-efficacy. One can perceive emotional arousal (increased heart rate, breathing rate, sweating, etc...) 
as excitement which may increase motivation and self-efficacy. The "excited" student feels they have the skills to succeed and feels excited to learn more about neuroanatomy. Alternately, another student might perceive this emotional arousal as fear or anxiety. The "fearful" student may process this feeling as not having the skills to complete the task at hand. Utilizing words to describe complex neuroanatomy problems as exciting and energizing intend to increase neuroanatomy self-efficacy by framing the emotional arousal towards excitement and away from fear.

Comparison of self-efficacy with self-confidence. Self-confidence, which is frequently mistaken for self-efficacy, must be clarified in order to distinguish these two constructs from each other. Confidence reflects a certain degree of certainty about a perception, event or an outcome (Merkle \& Van Zandt, 2006). For example, the degree of certainty about the accuracy of one's answers on an exam after taking an exam. Efklides (2011) described confidence as a metacognitive experience- one's self-assessment of their outcome. Self-efficacy can be considered one's metacognition about their abilities before an event occurs. For example, the amount of confidence a student feels in their abilities about a certain subject before the examination. Confidence can be thought of as one's metacognition of their outcome (and their abilities indirectly) after an event occurs. In the same example, self-confidence would be how certain the student correctly answered questions on the examination after the exam is over. Another important distinction is that self-efficacy varies in magnitude, generality and strength which has a theoretical basis in Social Learning Theory (Bandura, 1997) while confidence is often used without a theoretical basis lacking empirical data. 
Measurement Issues. One main issue with survey instruments designed to measure selfefficacy in medical education is that many scales are incongruent with self-efficacy theory in both domain specificity and conceptualization. Domain specificity is key as self-efficacy is defined as, "one's own perception of their ability to succeed at a task" (Bandura, 1977, p. 193) and the judgements one makes about their own abilities to complete tasks reflects their level of self-efficacy (Schunk, 1991). One's ability to complete a task depends on the domain, or situation, in which one finds themself. For example, a medical student may be adept at dissection skills in an anatomy cadaver laboratory and perceives their dissection skill self-efficacy as high in that situation. However, this medical student has no experience with brain dissection and, therefore, their perception of their dissections skills in a neuroanatomy laboratory setting may not be as high due to lack of experience in the neuroanatomy domain. One's own perception about their skills at a task depends on the domain, or situation, being asked. As such, Bandura (2006) describes how there is "no one measure fits all approach" (p. 307) when it comes to self-efficacy because of this domain specificity. For example, as is the case in this study, a measure of anatomy self-efficacy in medical students cannot be used for measuring medical student neuroanatomy self-efficacy. If no scale exists in the literature to measure the self-efficacy domain in which you are working, for example neuroanatomy, one must be developed.

Secondly, many medical education self-efficacy measures are incongruent with conceptualization of self-efficacy theory. According to self-efficacy researchers (Pajares, 1996; Bandura, 2006; Bandura, 2012; Schunk \& DiBenedetto, 2016), self-efficacy 
measures must contain three concepts to accurately measure self-efficacy. Self-efficacy measures must contain: (1) beliefs about future actions; (2) beliefs about skills; and, (3) task-specific items (Artino, 2012). Klassen and Klassen's (2018) review of ( $n=68)$ selfefficacy studies in medical education found $46 \%(n=31)$ were incongruent with selfefficacy theory. Items in these instruments were not future oriented, measured outcomes and not skills, evaluated alternate constructs (self-confidence, self-esteem, anxiety), or assessed general problem-solving knowledge instead of task specific skills. Examples of medical education self-efficacy measures that are both congruent and incongruent with self-efficacy theory will be examined below. Issues with specific items in these scales will be identified and discussed.

Domain specificity will be explored first. An educational intervention designed for physicians to assist patients in smoking cessation evaluated physician self-efficacy (Garg et al., 2007). Items specific to the smoking cessation domain were asked. For example, an item asked respondents to rate How confident are you that you can convey to your patients the information they need to quit smoking? (Garg et al., 2007). This is an example of a measurement scale item that was congruent with the domain. Alternately, a study by Francis et al. (2020) measuring self-efficacy of physician assistants in an operating room immersion simulation is an example of an incongruent domain item. One item assessing self-efficacy of the operating room simulation asked respondents to rate their confidence in the following statement, I can always manage to solve difficult problems if I try hard enough (Francis et al. (2020). This item reflects general problemsolving skills and not their perceived capabilities to solve problems while using an operating room simulator, in this example. 
Incongruency with self-efficacy conceptualization will be explored next. A measure of self-efficacy ought to contain items reflecting the self-efficacy construct and not another construct. One scale measuring medical student self-efficacy on the Objective Structured Clinical Examination (OSCE) asks students to rate their degree of anxiety you feel in anticipation of the OSCE (Mavis, 2001). This is an item measuring another construct, anxiety, and not self-efficacy. Self-efficacy scale items should also be futureoriented and task specific to align with self-efficacy theory (Pajares, 1996; Bandura, 2006; Bandura, 2012). Another study developed an educational intervention designed to improve skills of medical students in the geriatric population. The aim of Nagoshi et al. (2018) was to evaluate medical student self-efficacy of their skills with geriatric patients. A sample item from their self-efficacy scale asked respondents to rate the following, Geriatrics education was part of all four years of my medical education (Nagoshi et al., 2008). This item is not task specific, instead it is measuring the breadth of their medical training over four years. Additionally, this item is past oriented, and not future oriented to align with self-efficacy theory.

Lastly, scales with items congruent with self-efficacy conceptualization will be considered. To align with self-efficacy theory, scale items need to be task-specific, future oriented, and assess participants self-beliefs about skills. A study assessing the selfefficacy of medical student clinical-skills asked respondents to rate their confidence in the following, I am able to perform the skills we learned thus far on a patient (Turan et al., 2013). This item is task-specific to the skills already learned up to this point in the semester. Another scale of particular importance to this study from Burgoon et al. (2012), the anatomy self-efficacy scale, shows examples of alignment with self-efficacy theory. 
This scale is of particular importance to this study, because this is the scale from which the neuroanatomy self-efficacy scale was developed. Burgoon et al. (2012) shows an example of an item reflecting one's self-beliefs about their skills when asking students to rate how confident that, I can make the proper cuts in the cadaver as outlined in the lab manual. A second example from the same anatomy self-efficacy scale asked respondents to rate how confident that, I will be able to retain and recall anatomical knowledge for use in a clinical setting (Burgoon et al., 2012). The medical students in this study are in their first year of medical school and will be using their clinical skills in the future. Therefore, this item is an example of how a scale item can be aligned with the futureorientation aspect of self-efficacy theory.

In summary, there were few examples of complete scales with items that were congruent with self-efficacy theory in the medical education literature. As such, it is important to reflect on item development for a novel scale in medical education research as to align with self-efficacy theory from researchers (Pajares, 1996; Bandura, 2006; Bandura, 2012; Schunk \& DiBenedetto, 2016) in both domain and conceptualization. A literature search to-date found no psychometrically validated scales measuring neuroanatomy self-efficacy in medical students. However, a scale measuring anatomy self-efficacy by Burgoon et al. (2012), aligned with self-efficacy theory in both domain and conceptualization, was used as a model from which to develop our novel instrument. The aim of this study is to fill a gap in the medical education literature by developing a novel instrument to measure neuroanatomy self-efficacy and provide data for initial validity and reliability evidence for the instrument. Developing a scale to measure neuroanatomy self-efficacy will allow researchers to measure the level of neuroanatomy 
self-efficacy of medical students and study the effects of future educational interventions or curriculum changes designed to address it. A description of the methods used to develop the neuroanatomy self-efficacy scale in this study is described below.

\subsection{Methods.}

Target Population. Medical students are the target population for this study. A convenience sample of medical students enrolled at a large, public, Southeastern university $(n=625)$ were invited to participate in the online survey administered in Qualtrics ${ }^{\circledR}$ in 2018. A second cohort of first-year medical students $(n=162)$ were invited to participate in the study in the Fall 2019 semester.

\section{Item Development of Neuroanatomy Self-Efficacy Scale (NSES). Thirteen subjective} items comprise this scale (Appendix B) represents a person's beliefs in his or her own abilities to successfully complete a neuroanatomy task, based on the work of self-efficacy researchers (Pajares, 1996; Bandura, 2006; Bandura, 2012; Schunk \& DiBenedetto, 2016). Based on self-efficacy theory, this scale aims to measure three dimensions of perceived skill (magnitude), transferability (generality), and perseverance (strength) when learning neuroanatomy. Thirteen items were developed and scored based on the 16-item anatomy self-efficacy scale developed by Burgoon et al. (2012). Principal Axis Factoring of the original 16-item scale found one factor, anatomy self-efficacy, with an internal consistency (Cronbach's $\alpha$ ) between 0.90 to 0.96 for all sixteen items (Burgoon et al., 2012). We modified this scale for use as a neuroanatomy self-efficacy scale. Items were modified to focus on neuroanatomy, instead of anatomy, and to reflect the three 
dimensions of perceived skill (magnitude; 4 items), transferability (generality; 5 items), and perseverance (strength; 4 items). Respondents were asked to rate their confidence level on a 5-point Likert scale in doing 13 different neuroanatomy specific tasks ( $1=$ Not at All Confident, 2= Only a Little Confident, 3= Fairly Confident, 4= Very Confident and $5=$ Totally Confident). Neuroanatomy self-efficacy is scored as raw scores reported as a sum score of all items where lower scores signify lower self-rated neuroanatomy selfefficacy while higher scores represent greater self-reported neuroanatomy self-efficacy. For this scale, higher scores are indicative of higher neuroanatomy self-efficacy.

Demographic information. One demographic item, asking the respondent's gender, was informed by studies for inclusive measures of gender (Reisner et al., 2014; Bauer et al., 2017; Fraser, 2018). With $0.6 \%$ of the United States population (approximately 1.4 million adults) identifying as transgender (Flores et al., 2016), survey research has increasingly sought to include individuals that do not identify as a binary (male or female) gender. We relied on survey research in this area to inform our item development (Reisner et al., 2014; Bauer et al., 2017; Fraser, 2018) since there is currently no "gold standard" for gender inclusive survey items. We used the flow chart published in Fraser (2018) to determine the best practices for writing our gender item. Even though it was not necessary to identify transgender participants within our sample, we wanted to include non-cisgender survey items to respect our student diversity. The gender item provides an open response where respondents can self-describe their gender or prefer not to say. If our data determine we need a more inclusive gender item for future studies, Reisner et al. (2014) suggests a two question gender item- first, asking natal sex or gender status and 
second, current gender identity - which demonstrates acceptable validity $(r=0.47-0.63$, $p<0.0001$ ) and internal consistency (Cronbach's $\alpha=0.83$ ).

Instruments. In addition to the 13 NSES items and demographic information, the development of four additional instruments is described below. These instruments aim to measure premedical neuroscience experiences, neuroanatomy beliefs, emotions towards neuroanatomy, and final course grade. Scales specific to measuring previous neuroanatomy experiences, attitudes and emotions towards neuroanatomy have not yet been developed since neuroanatomy self-efficacy has not yet been explored in the research. Previous studies on recognizing emotion of language (Whissell, 1989; Whissell, 2009) and premedical experiences in gross anatomy (Forester et al., 2002) informed item development. The administration of these scales, concurrently with the NSES, will allow initial validation of this novel instrument. Demographic information and premedical neuroscience experience permit investigating the effects of gender and previous neuroscience experiences on neuroanatomy self-efficacy. Self-reported student final course grade will be correlated with NSES scores (at the beginning of the semester) to assess predictive validity. Neuroanatomy beliefs and attitudes towards neuroanatomy will be correlated with NSES scores to provide convergent validity evidence.

Premedical neuroscience and/or neuroanatomy exposure. Five items assessing premedical exposure to neuroanatomy were modified from the Forester et al. (2002) study assessing medical student premedical exposure to gross anatomy. The five dichotomous items $(0=N o, 1=Y e s)$ discerned previous exposure to neuroanatomy 
compared to neuroscience prior to starting medical school. The capacity of neuroanatomy experience was further differentiated between gross anatomy course content and anatomy and physiology course material. One last item asked respondents to confirm their neuroscience and/or neuroanatomy exposure in any capacity before starting medical school, in case their specific example was not listed. Definitions of both neuroanatomy and neuroscience were provided to avoid confusion. We defined neuroanatomy as anatomical connectivity within the brain and spinal cord. Neuroanatomy examples provided were the locations of cell bodies with associated brainstem nuclei, synapses, and decussation points of specific tracts (corticospinal tract, spinocerebellar tract, spinothalamic tract, etc...). The scale specifically describes lesion location isolation within the brain and spinal cord- utilizing clinical case scenarios- as neuroanatomy. We defined neuroscience as the molecular study of neurons. The study of synapses, axonal physiology, neurotransmitters with receptor physiology, and microscopic neuronal connectivity were provided as examples of neuroscience (Duque-Parra, 2002; Fischl et al., 2002; Rushmore et al., 2020). Care was taken to provide students a clear definition of neuroanatomy to help define the variable and preserve content validity of the scale. The researcher's intention is to discern premedical neuroanatomy from neuroscience experience.

Neuroanatomy Beliefs. This scale contains three, open-response items requiring students to provide three beliefs they have about the field of neurology and neurologists. It was decided against providing a series of common negative neurology beliefs and neurologist stereotypes, for which students would score on a Likert scale, to minimize both response 
bias and survey fatigue. Keeping a neutral tone of survey items prevents leading students into providing answers, students believe, the researchers may want to hear. Having students provide open responses may prevent survey fatigue from the other $(n=20)$ Likert-response items within the instrument. Scoring emotional words is based on the circumplex model of human affect which proposes emotional states arise from two main dimensions: valence (level of pleasantness) and arousal (level of alertness or activation) (Russell, 1980; Barrett, 2004; Posner et al., 2005). A literature search found the Dictionary of Affect in Language (DAL) (Whissell, 1989; Whissell, 2009) provides an evaluation (pleasantness) and arousal (activation level) score for emotional words based on a 3 -point scale in both the evaluation $(1=$ unpleasant, $2=$ in-between, $3=$ pleasant $)$ and arousal $(1=$ passive, $2=$ in-between, $3=$ active $)$ dimensions. The concurrent validity reported for the 2009 revised DAL is $r=0.71$ for evaluation and $r=0.45$ for activation based on words from the original dictionary (Whissell, 1989); test-retest reliability correlations were $r=0.80$ for evaluation and $r=0.69$ for activation (Whissell, 2009). The total score is reported as an average of evaluation and activation scores for all three words provided by respondents. A lower score suggests the respondent had more fearbased beliefs towards neurology, whereas higher scores correspond to higher positive beliefs towards neurology and neurologists.

Emotions towards neuroanatomy. This scale contains three, open-response items requesting students to self-reflect on how they felt about neuroanatomy at the start of medical school and to provide three strongest emotions they felt during that time. Similar to the neuroanatomy beliefs scale, providing an encompassing list of common positive 
and negative emotions, for which students would score on a Likert scale may lead to survey fatigue. Alternatively, if a student feels an emotion that is not on the list may lead to inaccurate reporting or response bias. Having students provide open responses may prevent survey fatigue from the other $(n=20)$ Likert-response items within the instrument. Scoring, similar to the subscale above, the researcher assigns a 3-point score for evaluation $(1=$ unpleasant, $2=$ in-between, $3=$ pleasant $)$ and arousal $(1=$ passive, $2=$ inbetween, $3=$ active) dimensions for each word provided by the student based on the Dictionary of Affect in Language (DAL) (Whissell, 1989; Whissell, 2009). The validity and reliability evidence for the DAL is reported in the above subscale. The total subscale score is reported as an average of evaluation and activation scores for all three words provided by respondents. A lower score suggests the respondent felt more fearful, or negatively, towards neuroanatomy at the beginning of medical school and higher scores suggests respondents felt excited, or more positive, towards neuroanatomy at the start of medical school.

Final course grade. One factual item asked students to self-report their anticipated (or received) grade in their first-semester medical student course. Scoring of the course grade was reported as 3= "Honors $(90-100 \%)$ ", $2=$ "Pass $(70-89 \%)$ ", or $1=$ "Fail $(69 \%$ or below)". A score of 0 was reported for students who dropped the course or provided an open-ended response to "Other Circumstance". There are two limitations of this item: (1) The neuroanatomy specific course outcome cannot be parsed out from the entire integrated course grade; and (2) Pass/fail nature of the course limits the available grading range for statistical analyses. 
Pilot. A pilot of 10 second-year medical student volunteers was conducted to assess content validity, online administration functionality in Qualtrics ${ }^{\circledR}$, and timing the length of the survey. Respondents were asked for their feedback assessing content validity: grammar, language, wording and question sensitivity. The pilot test assessed the length of time for pilot survey participation for informing participants of the survey's anticipated time commitment. Rewording of items was not necessary to preserve content validity of the scale, the online administration in Qualtrics ${ }^{\circledR}$ functioned without issue and the average time to complete the survey was 12 minutes.

Data Collection Procedures. The director of the integrated first semester medical school course sent an email invitation to participate in the online survey twice during the Fall 2018 and Fall 2019 semesters. First-year medical students were invited to complete the survey at the beginning of the semester (pre-course) and again at the end of the semester (pos-course). Ideally, two data points would be collected for each first-year medical student. Students were asked to provide a 5-digit code (suggested the last 5 digits of the student's phone number) for matching pre-course and post-course surveys. Upper-class medical students in 2018 were invited to participate in the online survey once at the end of the Fall 2018 semester. Completed surveys were exported from Qualtrics into a csv file in Microsoft Excel $^{\circledR}$. IP addresses were deleted from the files to protect anonymity. The files were imported into IBM SPSS ${ }^{\circledR}$ Version 26 where missing or non-complete surveys were deleted prior to analysis. Subscales were coded and scored as described above. 
Exploratory Factor Analysis. An exploratory factor analysis (EFA) with principal axis factoring was used to explore the underlying factor structure of the NSES instrument. EFA is recommended over a confirmatory factor analysis for initial scale development since it is possible our hypotheses regarding the latent construct's factor structure may not be correct (Carpenter, 2018). Principle axis factoring, assuming a causal model which generalizes to the population exists, was chosen over a principal components analysis which is typically used to reduce the number of items in a scale.

A Bartlett's test of sphericity and the Kaiser-Meyer-Olkin test (KMO) will be conducted to determine if the data for the 13-item NSES are suitable for exploratory factor analysis (EFA). A significance level of $p<0.01$ for the Bartlett's test of sphericity (Bartlett, 1950) and a KMO > 0.6 suggests the proportion of variance of the items are due to an underlying factor- which makes a factor analysis useful for these data. If initial EFA results suggest the three factors were correlated, the factor analysis will be run using an oblique rotation allowing for factor correlation; otherwise, the factor analysis will be conducting using an orthogonal rotation. Several measures will be used to determine the number of factors to retain for each scale: Eigenvalues greater than 1 according to the Kaiser-Guttman rule (Kaiser, 1991), the elbow of the scree plot, and parallel analysis with principal components analysis $\left(95^{\text {th }}\right.$ percentile criterion). The following criteria were used for retaining factors: (1) minimal pattern coefficients of 0.4 ; (2) nonrelevant factor pattern coefficients are less than 0.3; and (3) nonrelevant factor pattern coefficients were 0.2 less than the pattern coefficient on the retained factor (Thompson, 2004; Shuck et al., 
2017). A three-factor structure is anticipated based on the three-dimensional construct of self-efficacy (Bandura, 1977, 1986, 2001).

Internal reliability and validity analysis. The internal consistency reliability for the 13item scale will be examined by calculating Cronbach's alpha and a Guttman split-half reliability coefficient (Guttman, 1946; Cronbach, 1951; Clark \& Watson, 1995). For items that group together onto factors, subscale Cronbach's alphas for grouped items for each factor will be examined. The recommended acceptable 0.8 level for internal consistency will be used for all analyses (Cronbach, 1951; Clark \& Watson, 1995).

We will examine relationships (correlations) between the novel NSES scale with subscale scores to further provide validity evidence. Predictive validity assesses how well a measure (neuroanatomy self-efficacy) can predict future behavior (course grades). Selfefficacy has been shown to be a predictor of medical student outcomes in gross anatomy and histology (Burgoon et al., 2012 Forester et al., 2002; Peterson \& Tucker, 2005). The final course grade subscale will be correlated with pre-course NSES scale scores (in M1 students) to assess predictive validity. A positive correlation is anticipated from previous literature showing self-efficacy's predictive role in course grades.

Convergent validity assesses how well the score of another scale theoretically measures the same construct (neuroanatomy self-efficacy) concurrently. The scores of the neuroanatomy beliefs and emotions subscales will be correlated with the novel NSES scores. Negative emotions, particularly anxiety, and fear-based beliefs have been shown to be negatively correlated with student self-efficacy in the literature (van Dinther et al., 2011; Barrows et al., 2013; Razavi et al., 2018). Higher scores of the neuroanatomy 
emotions and beliefs subscales represent less fear-based, anxious emotions and beliefs; therefore, a positive correlation between NSES scores and neuroanatomy emotions and beliefs subscales is anticipated based on previous literature demonstrating an inverse relationship between fear-based emotions and self-efficacy.

Data Analysis. An independent $t$-test will be performed to investigate whether students with any premedical exposure to neuroscience or neuroanatomy exhibit higher neuroanatomy self-efficacy levels. Our hypothesis that students with premedical exposure to neuroscience or neuroanatomy will exhibit greater neuroanatomy self-efficacy is based on the literature on anatomy self-efficacy and premedical experiences (Foreseter et al., 2002; Peterson \& Tucker, 2005; Kondrashov et al., 2017). If significantly higher neuroanatomy self-efficacy levels are found, an analysis of variance (ANOVA) will be conducted to examine the differences between the specific types of premedical neuroanatomy and/or neuroscience exposure on neuroanatomy self-efficacy levels, when compared to no prior exposure. A Tukey's post-hoc test will be performed to isolate which type(s) of premedical neuroanatomy and/or neuroscience exposure significantly impacted medical student neuroanatomy self-efficacy. An effect size, Cohen's $d$, will be calculated to determine the magnitude of the differences. A Cohen's $d$ of 0.2 will be considered a small effect size, 0.5 a medium effect size and $\geq 0.8$ a large effect size (Cohen, 1992). The significance level used for hypothesis testing will be held at $p<0.05$.

To explore binary gender differences in neuroanatomy self-efficacy levels, an independent $t$-test will be performed. The significance level used for this analysis will be held at $p<0.05$. If a significant neuroanatomy self-efficacy difference between genders is 
found: (1) a chi-square analysis will be performed to ensure both genders had equivalent premedical neuroscience and/or neuroanatomy exposure; and subsequently (2) the impact of premedical neuroscience and/or neuroanatomy experience and binary gender on neuroanatomy self-efficacy levels will be further explored with a two-way analysis of variance (ANOVA). An effect size, Cohen's $d$, will be calculated to determine the magnitude of the observed difference. A Cohen's $d$ of 0.2 will be considered a small effect size, 0.5 a medium effect size and $\geq 0.8$ a large effect size (Cohen, 1992). The significance level used for hypothesis testing will be held at $p<0.05$.

\subsection{Results.}

4.4.1 Participants and Response Rate. All medical students enrolled at a large, public, Southeastern university $(n=625)$ were invited to participate in the online survey administered in Qualtrics ${ }^{\circledR}$ in 2018. A second cohort of first-year medical students ( $n=$ 162) were invited to participate in the study in the Fall 2019 semester. A total of 278 medical students voluntarily participated resulting in a $35.32 \%$ survey response rate. Of the total $(n=278)$ respondents, $60.07 \%(n=167)$ were first-year and $39.93 \%(n=111)$ were upper-class (second-, third- or fourth-year) medical students.

The study drew from a total student population of 787 medical students, 55\% male and $45 \%$ female, 23 of which were undergraduate neuroscience majors (2.92\%) (see Table 1). Publicly available data from the University of Louisville School of Medicine show the average age of all five entering classes (2015 - 2019) was 23.2. The average undergraduate overall GPA was 3.68 with the average Biology Chemistry Physics and Math (BCPM) GPA being 3.60. Racial demographics, reported as underrepresented in 
medicine, comprised $11 \%$ of entering students. It should be noted that the entering class of Fall 2017 had the lowest racial diversity (6\% underrepresented in medicine) while the Fall 2015 entering class profile comprised the highest diversity (14\%). The average age of the entering classes from 2015 to 2019 appears to decrease slightly from 24 to 23 , with the Fall 2019 with the youngest average age of 22.

4.4.2. Descriptive Statistics. Chi square tests were used to examine the binary gender differences between the survey participants and the general population of medical students at the university. Chi square tests indicated there were no significant gender differences between first-year medical student survey participants and first-year matriculating students in both the Fall $2018\left(X^{2}[1, n=60]=0.54, p=0.46\right)$ and Fall 2019 $\left(X^{2}[1, n=107]=1.7, p=0.19\right)$ semesters. A chi square test also showed no differences in binary gender between upper-class medical student survey participants compared to enrolled upper-class medical students at ULSOM in 2018, $X^{2}(1, n=111)=2.3, p=0.13$. Incomplete surveys $(n=45)$ were dropped from the analysis resulting in a total of $n=233$ surveys for data analysis. The sample was comprised of $48.56 \%(n=119)$ female, $44.24 \%$ $(n=113)$ male respondents, and $(n=1)$ non-binary gender student. The non-binary gender student was given their own category, however not included in the binary gender analysis.

4.4.3. Exploratory Factor Analysis Results. The results of measuring sampling adequacy of the 13-item NSES with Bartlett's test of sphericity $\left(\chi^{2}=1626.18, \mathrm{df}=21, \mathrm{p}\right.$ $<0.001)$ and the Kaiser-Meyer-Olkin test (0.92) suggests the data were suitable for 
exploratory factor analysis (EFA). The factor correlation matrix of the initial NSES EFA suggests the three factors were correlated $(0.66,0.61,0.62)$; therefore, we ran the factor analysis using an oblique rotation (Promax) allowing for factor correlation.

The pattern matrix (with structure coefficients in parentheses) and extraction communalities $\left(h^{2}\right)$ from the exploratory factor analysis (EFA) is shown below in Table 2. Item 5 ("I am confident that I can learn neuroanatomical terms and definitions") and item 9 ("I am confident that I can actively participate in neuroanatomical discussions") violated the second criterion. Both of these items showed nonrelevant factor pattern coefficients greater than 0.3 (Item $5=0.34$ and Item $9=0.32$ ) demonstrating crossloadings. However, since these nonrelevant factor pattern coefficients were greater than 0.2 less than the retained factor pattern coefficient, these two items were retained for this scale. These results suggest a three-factor solution with scale items 1-3 loading onto Factor 3, items 4-8 and item 13 loading onto Factor 1, and items 9-12 loading onto Factor 2. The communalities $\left(h^{2}\right)$ in Table 2 show the proportion of variance in the items that was explained by the factors. A description of the three-factor solution will be described below.

The proposed three factor solution for the 13-item neuroanatomy self-efficacy scale are Factor 1 (Transferability): Items 4-8 and 13, Factor 2 (Perseverance): Items 912, and Factor 3: Items 1-3 (Perceived Skill). The transferability of neuroanatomy selfefficacy was reflected in questions about using neuroanatomy knowledge for application; for example, "for use in a clinical setting" in Item 8 or "successfully on neuroanatomy written exams" in Item 6. Items 4-8 and 13 showed strong pattern coefficients, ranging from $0.73-0.89$. Perseverance of neuroanatomy self-efficacy, measured by Items $9-12$, 
require more persistence to solve the problem. For example, Item 12 asks respondents about their confidence about "neuroanatomically-based questions during clinical rotations". Clinical rotations are historically challenging for medical students; attending physicians routinely ask students successively more difficult questions that require perseverance to answer them while under duress. Items $9-12$ showed moderate to strong loadings, with pattern coefficients ranging from $0.55-0.93$. Lastly, the perceived skill aspect of neuroanatomy self-efficacy was described in Items 1-3. These items discuss skills medical students need to succeed in neuroanatomy. For example, Item 1 states, "I am confident that I can identify brain structures". Identification of anatomical structures in the brain and spinal cord are essential skills prior to understand more complex pathways and how to isolate lesions. Items $1-3$ also demonstrated strong pattern coefficients, ranging from $0.69-0.93$. However, Items 5 and 9 were troublesome in that they both demonstrated cross-loadings. A cross-loading is when a survey item demonstrates characteristics of being categorized with more than one factor and is demonstrated by nonrelevant pattern coefficient loadings greater than 0.3 . Since these nonrelevant factor pattern coefficients were greater than 0.2 less than the retained factor pattern coefficient, these two items were retained for this scale.

Further analysis of the eigenvalues, with the scree plot, and a parallel analysis show somewhat conflicting results demonstrating both two- and three-factor solutions. The eigenvalues of three factors are greater than 1.0 accounting for $76.11 \%$ of the total variance, which suggests retaining three factors. The sharp elbow of the scree plot (Fig. 3 ) is observed at the second factor, suggesting retaining only two factors. However, a parallel analysis with principal components analysis $\left(95^{\text {th }}\right.$ percentile criterion $)$ supports a 
three-factor solution with three factor eigenvalues greater than 1.0. Two out of the three criteria for retaining factors suggest a three-factor solution for this scale.

4.4.4. Internal Consistency, Validity and Reliability Results. The internal consistency reliability for items that grouped together was examined. The Cronbach's alpha for Items 1,2 and 3 on factor 1 (Transferability) was 0.925 . Items 9 -12 that grouped together on factor 2 (Perseverance) showed a Cronbach's alpha of 0.894. The Cronbach's alpha of items 4-8 and 13 on Factor 3 (Perceived Skill) was 0.81 . These levels are above the recommended acceptable 0.8 level (Cronbach, 1951; Clark \& Watson, 1995) for internal consistency. The entire 13-item NSES demonstrated a Cronbach's alpha also above the acceptable 0.8 level $(\alpha=0.94)$. A Guttman split-half reliability coefficient was calculated to be 0.871 (Guttman, 1946).

Other instruments were administered along with the NSES instrument to assess the predictive and convergent validity of the novel NSES scale. These other scales included self-reported student grade, emotions towards neuroanatomy, beliefs towards neuroanatomy, and attitudes towards neuroanatomy. We correlated pre-course neuroanatomy self-efficacy scores with matched post-course self-reported student grades for first-year medical students. We observed a significant positive correlation $(r=0.55$, $p=0.01)$. The emotions towards neuroanatomy, beliefs towards neuroanatomy and attitudes towards neuroanatomy subscales were used to correlate with the NSES scores. A significant positive correlation between neuroanatomy self-efficacy and each of the subscales (emotions towards neuroanatomy $r=0.329, p<0.001$; beliefs towards 
neuroanatomy $r=0.164, p=0.006$; attitudes towards neuroanatomy $r=0.29, p<0.001$ ) was observed.

\subsubsection{Neuroanatomy self-efficacy differences and premedical neuroscience and/or}

neuroanatomy experiences. To investigate whether any premedical exposure to neuroscience or neuroanatomy affects neuroanatomy self-efficacy levels of medical students, an independent $t$-test was performed. Less than half $(43.8 \%, n=102)$ of our participants self-reported any previous exposure to neuroscience and/or neuroanatomy exposure and 56.2\% $(n=131)$ reported any previous neuroscience and/or neuroanatomy experience in any capacity prior to starting medical school (see Figure 4). Our students demonstrated, on average, a moderate level of neuroanatomy self-efficacy $(M=41.14$, $S D=9.9, n=233$ ); a significant difference in neuroanatomy self-efficacy levels was found between students who reported any previous exposure to neuroanatomy or neuroscience before starting medical school and students who reported none $(t(231)=-2.61, p=0.01$; Figure 4). Further, a Cohen's effect size $(d=0.34)$ suggested a small practical significance.

To further investigate differences between the specific types of premedical neuroanatomy or neuroscience exposure on neuroanatomy self-efficacy levels, when compared to no prior exposure, an analysis of variance (ANOVA) was performed. A significant difference in neuroanatomy self-efficacy levels between the groups, $F(5,227)$ $=4.01, p=0.002$ (Figure 5). A Tukey's post-hoc test was performed to isolate which type of premedical neuroanatomy or neuroscience exposure showed significant differences in neuroanatomy self-efficacy (when compared to students with no prior exposure). As 
expected, students with both premedical molecular neuroscience and neuroanatomy courses (NSAC) demonstrated the greatest neuroanatomy self-efficacy when compared to students with no premedical experience, NPN, $p=0.001$ (Figure 5). This mean difference showed a Cohen's $d=0.52$ suggesting a moderately practical significance of this category of premedical experience. Interestingly, neither a premedical molecular neuroscience course (NSC) or a premedical neuroanatomy course (NAC) showed a significant increase in neuroanatomy self-efficacy levels (Figure 5). However, indirect premedical neuroanatomy exposure as part of a premedical gross anatomy course (GR) showed significantly increased neuroanatomy self-efficacy $(p=0.01)$, with a moderate effect size (Cohen's $d=0.50$ ), compared to students with no premedical exposure (NPN). Students with indirect neuroanatomy exposure from having completed a premedical anatomy and physiology course (AP) demonstrated similar neuroanatomy self-efficacy to students with no previous neuroscience or neuroanatomy exposure (NPN).

\subsubsection{Gender differences in neuroanatomy self-efficacy and premedical}

neuroscience and neuroanatomy experiences. To investigate binary gender differences in neuroanatomy self-efficacy levels, an independent $t$-test was performed. There was a significant difference in neuroanatomy self-efficacy levels for gender, $t(230)=3.99, p<$ 0.001 , with a medium effect size (Cohen's $d=0.52$ ) suggesting moderate practical significance; male participants demonstrated higher levels of neuroanatomy self-efficacy, $M=42.96, S D=9.88, n=113$, than female participants, $M=37.87, S D=9.47, n=119$

(Figure 6). In order to determine if this was possibly due to male students having significantly more premedical neuroscience or neuroanatomy experience, a chi-square 
analysis was performed. Of students reporting any premedical exposure to neuroscience or neuroanatomy before starting medical school, $47.3 \%$ were male and $52.7 \%$ were female; male students did not have significantly more premedical neuroscience and/or neuroanatomy exposure, $X^{2}(2, n=232)=1.52, p=0.47$.

To further investigate the individual differences of premedical neuroscience experience and binary gender on neuroanatomy self-efficacy levels, a two-way analysis of variance (ANOVA) was performed. As reported above, a significant difference in neuroanatomy self-efficacy levels between the genders, $F(2,228)=9.87, p=0.001$, was found. Interestingly, male students with premedical neuroscience and/or neuroanatomy did not show significantly higher levels of neuroanatomy self-efficacy compared to their male colleagues without any experience $(p=0.93)$ (see Figure 7). However, female students with premedical neuroscience and/or neuroanatomy experience exhibited significantly higher neuroanatomy self-efficacy than their non-experienced female colleagues $(p=0.02)$.

4.5 Discussion. Our novel data show two distinct premedical neuroscience experiences that significantly impact first-year medical student neuroanatomy self-efficacy (when compared to no premedical experience): (1) combined premedical molecular neuroscience and neuroanatomy course enrollment, and (2) indirect neuroanatomy exposure as part of a premedical gross anatomy course. Our findings that any premedical neuroscience or neuroanatomy experience is associated with higher neuroanatomy selfefficacy in our first-year medical students is consistent with previous studies on premedical educational experiences and self-efficacy in gross anatomy (Forester et al., 
2002; Peterson \&Tucker, 2005; Kondrashov et al., 2017). These novel findings add to the breadth and depth of literature on premedical neuroanatomy experiences and selfefficacy.

Our results show students with previous enrollment in both premedical molecular neuroscience and neuroanatomy courses demonstrated the highest neuroanatomy selfefficacy scores. Our anticipation that these premedical neuroanatomy and neuroscience experiences may contribute to neuroanatomy self-efficacy is based on Bandura's (1977, 2001) work on self-efficacy and Social Cognitive Theory. As Bandura (1977) describes, performance mastery is the greatest source of self-efficacy. Medical students that have previously worked through both neuroanatomy and neuroscience material would have performance mastery experience in both areas, theoretically contributing to higher neuroanatomy self-efficacy scores. Our data supports this hypothesis. Additionally, our data show neither a premedical molecular neuroscience course nor premedical neuroanatomy course individually resulted in significantly higher neuroanatomy selfefficacy scores than no premedical experience. Previous studies show medical students have particularly limited abilities of self-assessment in both learning and performance (Edwards et al., 2003; Blanch-Hartigan et al., 2011; Hall et al., 2016) especially early in their medical school career (Swandon \& Finn, 2004). High achieving medical students tend to underestimate their abilities while low-achieving medical students overestimate their abilities (Edwards et al., 2003; Blanch-Hartigan, 2011). It would be anticipated that medical students with premedical neuroanatomy experience would score higher on neuroanatomy self-efficacy surveys through previous performance mastery experiences (Bandura, 1977). Perhaps these data are reflecting this limited self-assessment ability; 
higher performing medical students, even with premedical neuroanatomy experience, are underestimating their own abilities.

Students with indirect neuroanatomy experience, as part of a premedical gross anatomy course, showed the second highest levels of neuroanatomy self-efficacy. It was unexpected that gross anatomy experience outperformed direct study of individual neuroanatomy and neuroscience in premedical courses. One may anticipate neuroanatomy-specific performance mastery may contribute to higher neuroanatomy self-efficacy from Bandura's $(1977,2001)$ work on direct performance mastery as the greatest source of self-efficacy. It may be that premedical gross anatomy, with a cadaveric laboratory, provides a greater source of performance mastery than a standalone neuroanatomy course. Many gross anatomy courses study peripheral nerves and plexuses, including cranial nerves, as part of the course. Possibly studying the peripheral nervous system pathways, and visualizing nerves, enhances a student's understanding of central pathways by allowing the learner to connect the two. Previous studies with medical students in gross anatomy show cadaveric-specific experiences provide higher anatomy self-efficacy (Burgoon et al., 2012) and medical student success (Foreseter et al., 2002; Peterson \& Tucker, 2005; Kondrashov et al., 2017). In lieu of this finding, a future study may determine which aspects of the gross anatomy experience (ex. dissection compared to prosection, cranial nerve pathways, brachial plexus, etc...) best contribute to neuroanatomy self-efficacy.

Students with indirect neuroanatomy experience, as part of an anatomy and physiology course, show similar neuroanatomy self-efficacy levels to students having no neuroscience or neuroanatomy experience. Most anatomy and physiology courses review 
basic neuroscience (molecular details of synaptic transmission and nerve physiology) with little to no neuroanatomy exposure. It was not surprising that this secondary neuroscience experience showed lower neuroanatomy self-efficacy as compared to gross anatomy-where nerve pathways and some neuroanatomy content is delivered within the course. However, it was surprising that it provided no benefit when compared to students with no premedical neuroscience. It was anticipated some performance mastery (Bandura, 1977) would lead to an increase in neuroanatomy self-efficacy when compared to no experience. Many anatomy and physiology courses are delivered in a year-long course with two parts: part one in the fall semester and part two in the spring. Perhaps anatomy and physiology enrollees only took one part of the year-long course and did not encounter the neuroscience content. Or, the delivery of the neuroscience content was minimal and had no effect on the neuroanatomy self-efficacy level of the student.

An interesting secondary finding of this study was premedical exposure to neuroscience and/or neuroanatomy appears to increase neuroanatomy self-efficacy of female medical students while their male colleagues do not show the same pattern of improved neuroanatomy self-efficacy with premedical experiences. This finding is interesting considering the gender disparity in neurology: there are fewer women in the fields of neurology, neurosurgery and neuroradiology. A 2014 study from the Journal of the American Medical Association (JAMA) reports 11.9\% of full professor appointments were women compared to $28.6 \%$ men in a cross-sectional database of 91,073 medical school faculty appointments- a 16.7\% difference. A more recent 2018 study of 1,712 academic neurologists showed that only 30.8\% $(n=528)$ were women (McDermott et al., 2018). While a gender disparity exists in many medical school faculty departmental 
appointments, a particularly large gender gap exists in neurological medicine. Men outnumbered women at all academic faculty ranks and the disparity grew with advancing rank, but only $13.8 \%$ full professor neurology appointments were women compared to 86.2\% men (McDermott et al., 2018)- a 72.4\% difference. Given our findings that premedical neuroscience and/or neuroanatomy experiences appear to preferentially affect the neuroanatomy self-efficacy of female medical students, along with the gender disparity and overall declining interest in neurology careers, targeting female students to enter neurology may bridge these gaps. However, more study is needed on the impact of neuroanatomy self-efficacy on medical school neuroanatomy performance and one's interest to pursue neurology as a career.

Our initial data shows the neuroanatomy self-efficacy scale (NSES) is a valid and reliable scale. Convergent and predictive validity were shown through significant correlations with scales (Neuroanatomy Emotions, Neuroanatomy Beliefs, and SelfReported Student Grade) administered concurrently with the NSES. The entire 13-item NSES demonstrated strong reliability through a Cronbach's $\alpha(0.94)$ and a Guttman split-half reliability coefficient ( 0.87 ) above the acceptable 0.8 level. The construct validity results, shown through an exploratory factor analysis (EFA), suggest this survey may benefit from further editing. Items 5 and 9 were troublesome in that they both demonstrated cross-loadings. A cross-loading is when a survey item demonstrates characteristics of being categorized with more than one factor and is shown by nonrelevant pattern coefficient loadings greater than 0.3 . Since these nonrelevant factor pattern coefficients were greater than 0.2 less than the retained factor pattern coefficient, these two items were retained for this scale. First, our data showed a nonrelevant loading 
of Item 5 (0.342) on Factor 3 (Perceived Skill). This item showed primary characteristics of Factor 1 (Transferability) with a pattern coefficient of 0.729. The author's intention was Item 5 to primarily load onto to Factor 3 (Perceived Skill). This was to ensure a balanced number of items, loading onto each of the three factors, for a 13-item measure $($ Factor $1($ Transferability $)=5$ items, Factor $2($ Perseverance $)=4$ items, and Factor 3 (Perceived Skill) $=4$ items). The author suggests the deletion of "and definitions" part of the text of Item 5 . The way this item was written may imply anatomical terms would be used for transferability of this skill into an action, such as an explanation on a written examination. Additionally, adding Item 14 to read "I am confident that I can learn progressively more difficult neuroanatomical terms throughout the semester" may emphasize the development progressively increasing perceived skill level necessary to complete the task as the semester proceeds. Second, item 9 showed a primary loading (0.552) on Factor 2 (Perseverance) with a nonrelevant loading (0.319) on Factor 1 (Transferability). Editing Item 9 to read, "I am confident that I can be an active participant in difficult or complex neuroanatomical discussions" (with difficult or complex italicized) may shift the emphasis of this item to the perseverance aspect of this skill. Adding the words "difficult or complex" suggests a level of determination required to be an active part of the discussion. One who perseveres does not give up easily when discussions become challenging. Combined with the conflicting results of the scree plot, eigenvalues and the factor pattern matrix, another round of data collection and EFA- with the reworded and additional items- is suggested for further analysis. Another EFA may differentiate which item wording (either Item 5 or Item 14) may best load onto Factor 3 (Perceived Skill) with minimal cross-loadings for a more balanced 13-item scale. 
Additionally, a second round of EFA may also determine if rewording item 9 improves its primary loading on Factor 2 (Perseverance). Although a further round of EFA may further strengthen the construct validity of the novel NSES scale, the strong convergent validity, predictive validity, and reliability evidence suggest that the NSES scale in its current state is a valid and reliable tool.

In summary, premedical exposure to molecular neuroscience and neuroanatomy, particularly as part of a gross anatomy course, appears to be associated with higher neuroanatomy self-efficacy in first-year medical students. This premedical experience also appears to preferentially impact female students. Considering the gender disparity in neurology, and associated decline in neurologists, these findings support youth STEM neuroscience initiatives to give students increased pre-medical experiences with neuroscience and neuroanatomy. Additionally, the development of the neuroanatomy self-efficacy scale from this study will be used to evaluate the effects of a learning intervention designed to improve neuroanatomy self-efficacy, discussed in Chapter 5 of this dissertation. 


\section{TABLE 4.1}

\begin{tabular}{|c|c|c|c|c|c|c|c|c|c|c|}
\hline Class & $N$ & $\begin{array}{c}\text { NM } \\
(n)\end{array}$ & & $\begin{array}{l}\mathbf{G} \\
\mathbf{P} \\
\mathbf{A}\end{array}$ & $\begin{array}{c}\text { BCPM } \\
\text { GPA }\end{array}$ & MCAT & GeI & der & Age & UIM \\
\hline 2015 & 154 & 3 & & 3.66 & 3.58 & $*$ & $58 \%$ & $42 \%$ & 24 & $14 \%$ \\
\hline 2016 & 156 & 2 & & 3.70 & 3.63 & 507 & $52 \%$ & $48 \%$ & 24 & $12 \%$ \\
\hline 2017 & 155 & 2 & & 3.73 & 3.65 & 507 & $54 \%$ & $46 \%$ & 23 & $6 \%$ \\
\hline 2018 & 160 & 8 & & 3.65 & 3.55 & 508 & $57 \%$ & $43 \%$ & 23 & $11 \%$ \\
\hline 2019 & 162 & 8 & & 3.68 & 3.59 & 508 & $54 \%$ & $46 \%$ & 22 & $12 \%$ \\
\hline Total: & 787 & 23 & Avg: & 3.68 & 3.60 & 507.5 & $55 \%$ & $45 \%$ & 23.2 & $11 \%$ \\
\hline
\end{tabular}




\section{TABLE 4.1 Study Population: Class profile demographics for ULSOM entering}

classes 2015 to 2019. $N=$ number of matriculated students; $N M=$ Number of

neurobiology or neuroscience majors; $G P A=$ overall grade point average (4.0 scale);

$B C P M G P A=$ overall Biology, Chemistry, Physics and Mathematics grade point average

(4.0 scale); $M C A T=$ Medical College Admissions Test scores (* Entering class 2015

scores were graded on the old system and not included in the average. Entering Class

2015 MCAT scoring= Verbal: 9.68, Physical Science: 9.53; Biological Science 10.05);

Gender $=$ binary gender listed as male and female; Age $=$ average age of entering class;

$U I M=$ underrepresented in medicine reflecting the racial diversity of entering class. 


\section{TABLE 4.2}

\begin{tabular}{|c|c|c|c|c|c|}
\hline $\begin{array}{c}\text { All Medical } \\
\text { Student } \\
\text { Matriculants }\end{array}$ & GPA $^{\mathbf{1}}$ & $\begin{array}{c}\text { BCPM }^{\text {GCPA }} \\
\text { GPA }^{\mathbf{1}}\end{array}$ & MCAT $^{\mathbf{1}}$ & \multicolumn{2}{|c|}{ Gender $^{\mathbf{2}}$} \\
\hline 2016 & 3.70 & 3.64 & 508.7 & $50.2 \%$ & $49.8 \%$ \\
\hline 2017 & 3.71 & 3.64 & 510.4 & $49.3 \%$ & $50.7 \%$ \\
\hline 2018 & 3.72 & 3.65 & 511.2 & $48.4 \%$ & $51.6 \%$ \\
\hline 2019 & 3.73 & 3.66 & 511.5 & $47.6 \%$ & $52.4 \%$ \\
\hline
\end{tabular}




\section{TABLE 4.2 MCAT Scores, GPAs, and Gender Composite of Matriculants to US}

Medical Schools, 2016 through 2019. Data from the Association of American Medical Colleges (AAMC) showing the characteristics of total incoming matriculants to US Medical School for the same timeframe as this study. $G P A=$ overall grade point average (4.0 scale); $B C P M G P A=$ overall Biology, Chemistry, Physics and Mathematics grade point average (4.0 scale); $M C A T=$ Medical College Admissions Test scores; Gender= binary gender listed as male and female.

\footnotetext{
${ }^{1}$ Association of American Medical Colleges. 2020. MCAT Scores and GPAs for Applicants and Matriculants to U.S. Medical Schools, 2016-2017 through 2020-2021. [https://www.aamc.org/data-reports/students-residents/interactive-data/2020-factsapplicants-and-matriculants-data]. Accessed [28 February 2020]
}

${ }^{2}$ Association of American Medical Colleges. 2020. Applicants, First-Time Applicants, Acceptees, and Matriculants to U.S. Medical Schools by Sex, 2011-2012 through 20202021. [https://www.aamc.org/data-reports/students-residents/interactive-data/2020-factsapplicants-and-matriculants-data]. Accessed [28 February 2020] 


\section{TABLE 4.3}

\begin{tabular}{|c|c|c|c|c|}
\hline & \multicolumn{3}{|c|}{ Pattern Matrix } & Communalities \\
\hline Item & $\begin{array}{c}\text { Factor } \\
1 \\
\end{array}$ & $\begin{array}{c}\text { Factor } \\
2\end{array}$ & $\begin{array}{c}\text { Factor } \\
3\end{array}$ & $h^{2}$ \\
\hline $\begin{array}{l}\text { 2. I am confident that I can identify the } \\
\text { orientation of brain slices (ex: sagittal, } \\
\text { horizontal, coronal) }\end{array}$ & $\begin{array}{l}-0.10 \\
(0.45)\end{array}$ & $(0.49)$ & $\begin{array}{c}\mathbf{0 . 9 3} \\
(0.85)\end{array}$ & 0.74 \\
\hline $\begin{array}{l}\text { 1. I am confident that I can identify } \\
\text { brain structures }\end{array}$ & $(0.49)$ & $\begin{array}{c}0.17 \\
(0.58)\end{array}$ & $\begin{array}{c}\mathbf{0 . 7 2} \\
(0.79)\end{array}$ & 0.64 \\
\hline $\begin{array}{l}\text { 3. I am confident that I can correctly } \\
\text { pronounce neuroanatomical terms }\end{array}$ & $(0.55)$ & $(0.54)$ & $\begin{array}{c}\mathbf{0 . 6 9} \\
(0.78) \\
\end{array}$ & 0.62 \\
\hline $\begin{array}{l}\text { 8. I am confident that I will be able to } \\
\text { retain and recall neuroanatomical } \\
\text { knowledge for use in a clinical } \\
\text { setting }\end{array}$ & $\begin{array}{c}\mathbf{0 . 8 9} \\
(0.86)\end{array}$ & $(0.57)$ & $\begin{array}{l}-0.13 \\
(0.45)\end{array}$ & 0.74 \\
\hline $\begin{array}{l}\text { 6. I am confident that I can perform } \\
\text { successfully on neuroanatomy written } \\
\text { exams }\end{array}$ & $\begin{array}{c}\mathbf{0 . 8 8} \\
(0.87)\end{array}$ & $(0.58)$ & $(0.49)$ & 0.76 \\
\hline $\begin{array}{l}\text { 4. I am confident that I can } \\
\text { neuroanatomy relationships (i.e., how } \\
\text { one item relates to another in position } \\
\text { in the brain) }\end{array}$ & $\begin{array}{c}\mathbf{0 . 7 5} \\
(0.84)\end{array}$ & $(0.59)$ & $\begin{array}{c}0.16 \\
(0.61)\end{array}$ & 0.73 \\
\hline $\begin{array}{l}\text { 7. I am confident that I can perform } \\
\text { successfully on neuroanatomy lab } \\
\text { practical exams }\end{array}$ & $\begin{array}{c}\mathbf{0 . 7 3} \\
(0.80)\end{array}$ & $(0.55)$ & $\begin{array}{c}0.11 \\
(0.55)\end{array}$ & 0.64 \\
\hline $\begin{array}{l}\text { 13. I am confident that I can learn the } \\
\text { neuroanatomical content of this course }\end{array}$ & $\begin{array}{c}\mathbf{0 . 7 9} \\
(0.75) \\
\end{array}$ & $(0.51)$ & $\begin{array}{l}-0.17 \\
(0.36) \\
\end{array}$ & 0.57 \\
\hline $\begin{array}{l}\text { 5. I am confident that I can learn } \\
\text { neuroanatomical terms and definitions }\end{array}$ & $\begin{array}{c}0.73 \\
(0.83) \\
\end{array}$ & $\begin{array}{c}0.16 \\
(0.53) \\
\end{array}$ & $\begin{array}{l}0.34^{*} \\
(0.69) \\
\end{array}$ & 0.75 \\
\hline $\begin{array}{l}\text { 12. I am confident that I can } \\
\text { successfully answer } \\
\text { neuroanatomically-based questions } \\
\text { during clinical rotations }\end{array}$ & $(0.59)$ & $\begin{array}{c}\mathbf{0 . 9 3} \\
(0.90)\end{array}$ & $(0.54)$ & 0.82 \\
\hline $\begin{array}{l}\text { 11. I am confident that I can describe } \\
\text { neuroanatomical structures of the brain } \\
\text { to a non-medical person }\end{array}$ & $(0.52)$ & $\begin{array}{c}\mathbf{0 . 6 7} \\
(0.77)\end{array}$ & $\begin{array}{c}0.21 \\
(0.59)\end{array}$ & 0.61 \\
\hline $\begin{array}{l}\text { 10. I am confident that I can locate } \\
\text { clinically relevant neuroanatomical } \\
\text { lesions in the human brain }\end{array}$ & $(0.60)$ & $\begin{array}{c}\mathbf{0 . 7 9} \\
(0.85)\end{array}$ & $(0.56)$ & 0.73 \\
\hline $\begin{array}{l}\text { 9. I am confident that I can actively } \\
\text { participate in neuroanatomical } \\
\text { discussions }\end{array}$ & $\begin{array}{l}0.32 * \\
(0.70)\end{array}$ & $\begin{array}{c}\mathbf{0 . 5 5} \\
(0.77)\end{array}$ & $(0.56)$ & 0.66 \\
\hline
\end{tabular}




\section{TABLE 4.3 Exploratory Factor Analysis (Principal Axis Factoring) Pattern Matrix.}

Pattern coefficients in bold have values greater than 0.4 for primary loading of an item on that factor (structure coefficients are in parentheses). Items denoted with an asterisk $\left(^{*}\right)$ : Item 5 and Item 9 show cross-loadings with nonrelevant factor loadings greater than 0.3 ; $h^{2}=$ communalities of the measured variables; Promax rotation was used 


\section{FIGURE 4.1}

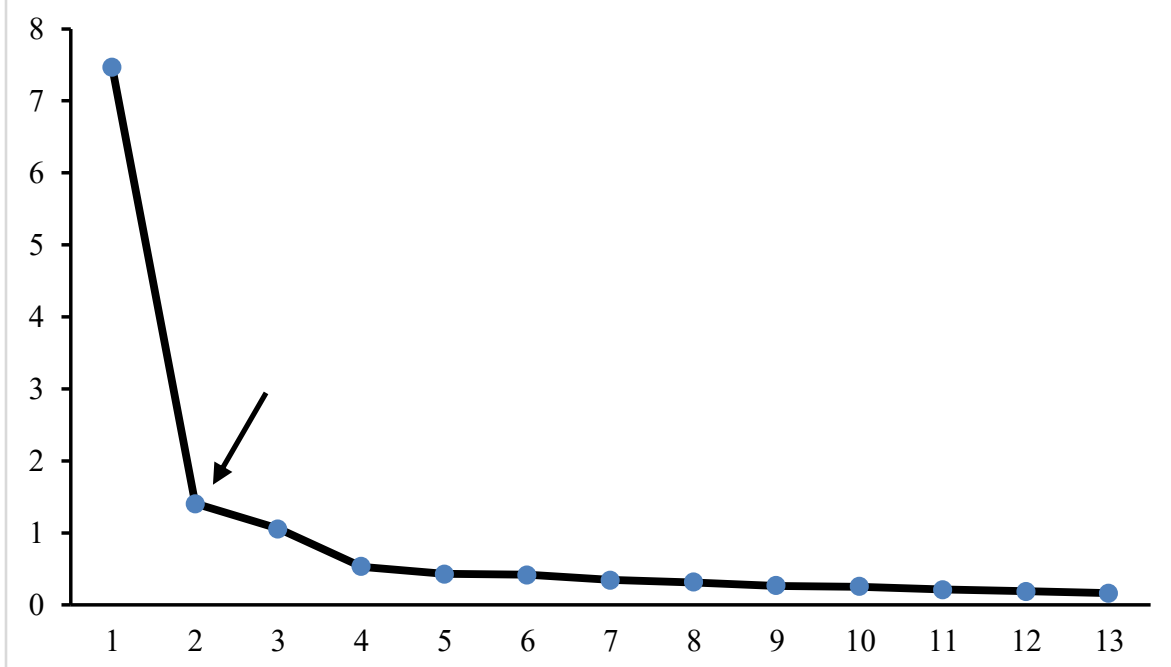


FIGURE 4.1. Scree Plot. Eigenvalues plotted for each factor shows the elbow at the second factor (arrow), suggesting a two-factor solution. A parallel analysis with principal components analysis $\left(95^{\text {th }}\right.$ percentile criterion) and exploratory factor analysis support a three-factor solution along with three factor eigenvalues greater than 1.0. Two out of the three criteria for retaining factors suggest a three-factor solution for this scale. 


\section{FIGURE 4.2}

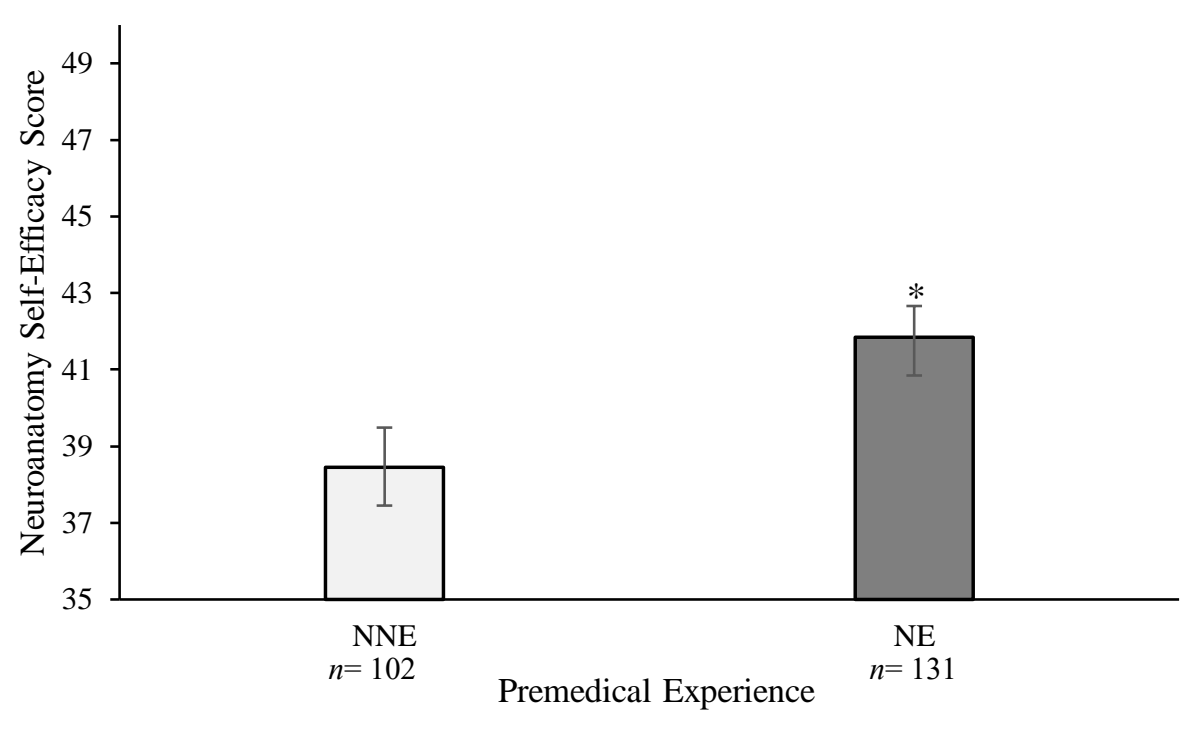


FIGURE 4.2 Neuroanatomy self-efficacy scores (reported values are mean \pm S.E.) based on premedical experience with neuroscience or neuroanatomy. The asterisk (*) indicates a significant difference $(p=.01)$ in neuroanatomy self-efficacy levels of students that were previously exposed to any level of neuroanatomy and/or neuroscience (NE) compared to students without prior experience (NPN). 


\section{FIGURE 4.3}

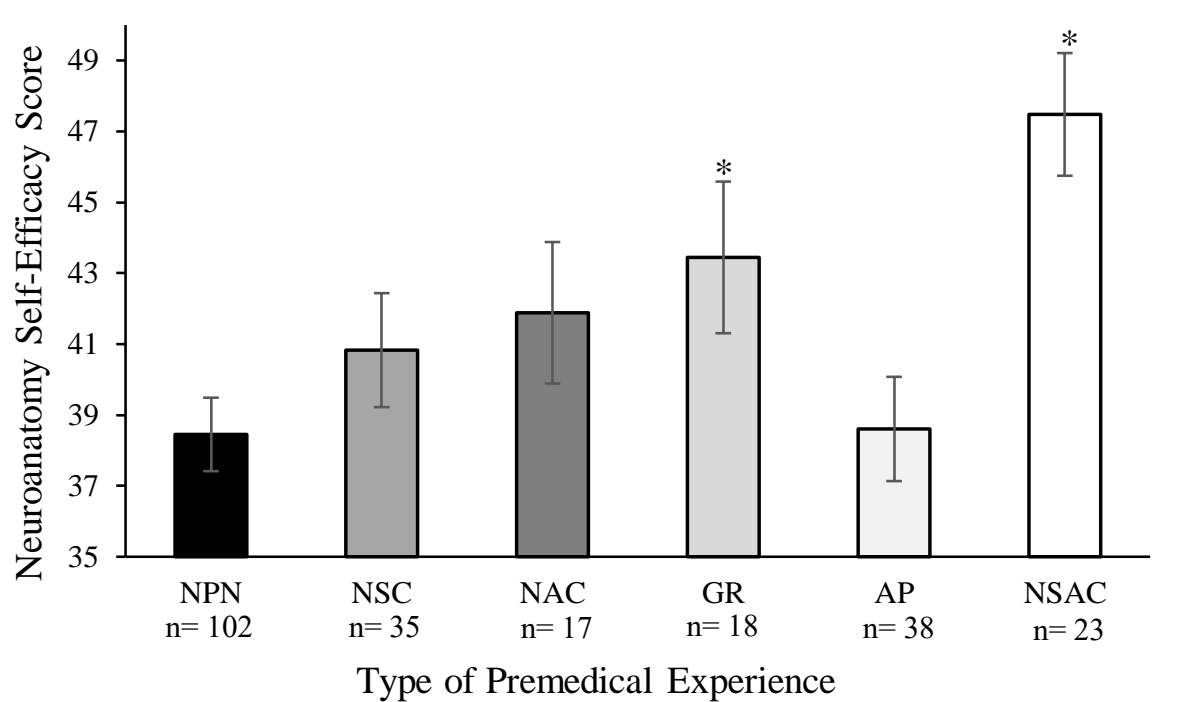


FIGURE 4.3 Differences in neuroanatomy self-efficacy scores (reported values are mean \pm S.E.) according to the type of premedical neuroanatomy experience. The asterisk $(*)$ indicates a significant difference $(p<0.05)$ in neuroanatomy self-efficacy levels of students that were previously exposed to neuroanatomy secondarily as part of a premedical gross anatomy course (GR; $p=0.04)$ or those students who took both premedical neuroscience and neuroanatomy courses (NSAC; $p=0.001)$ when compared to students without any prior experience (NPN). 
FIGURE 4.4

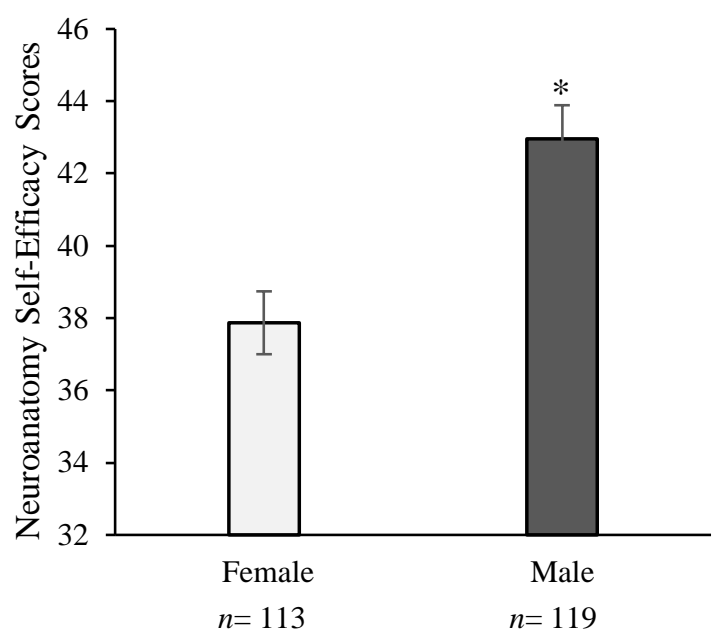


FIGURE 4.4. Neuroanatomy self-efficacy scores (reported values are mean \pm S.E.) based on binary gender. The asterisk $(*)$ indicates a significant difference $(p<0.001)$ in neuroanatomy self-efficacy levels of male students compared to female students. 


\section{FIGURE 4.5}

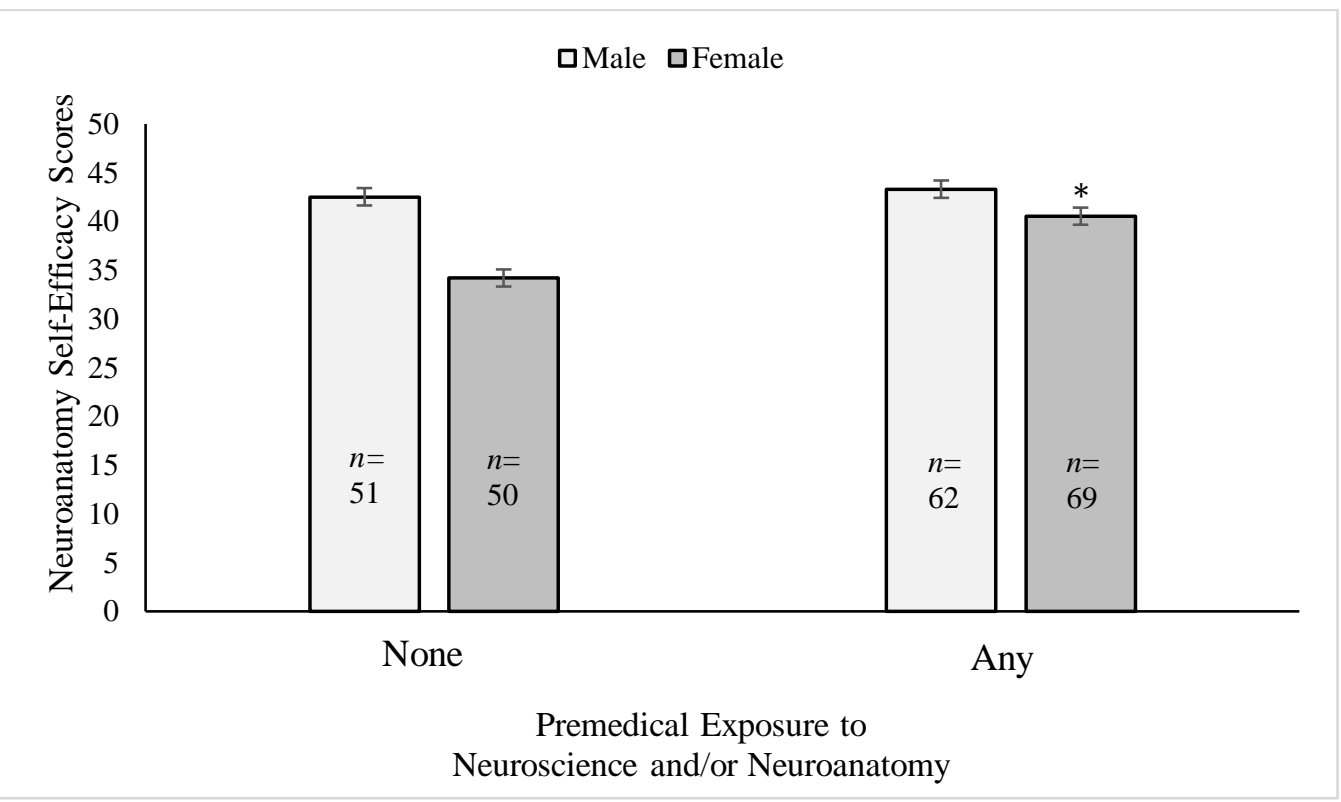


FIGURE 4.5. Neuroanatomy self-efficacy scores (reported values are mean \pm S.E.) according to gender and premedical neuroscience exposure. The asterisk (*) indicates a significant difference $(p=0.02)$ in neuroanatomy self-efficacy levels of female students that were previously exposed to any neuroanatomy and/or neuroscience compared to female students without any prior experience. 


\title{
CHAPTER 5
}

\author{
COMPARING THE EFFECTS OF A NON-ADAPTIVE TO AN ADAPTIVE \\ ELEARNING INTERVENTION ON FIRST-YEAR MEDICAL STUDENT \\ NEUROANATOMY SELF-EFFICACY AND NEUROANXIETY IN AN \\ INTEGRATED CURRICULUM
}

5.1 Introduction. Anxiety towards learning neuroanatomy in medical school may be negatively influencing medical student success in neuroanatomy (Hudson et al., 2006; Anwar et al., 2015; Shiels et al., 2017; Javaid et al., 2018; Sotgiu et al., 2020). This key neuroanxiety issue, originally called neurophobia, is extensively published in the literature (Schon et al., 2002; Flanagan et al., 2007; Youssef, 2009; Zinchuk et al., 2010; McCarron et al., 2014; Abulaban et al., 2015). We proposed neurophobia may more accurately be described as neuroanxiety as discussed in Chapter 3 of this dissertation. Concurrently, neuroanatomy education is becoming "increasingly marginalized in medical school curricula" (Mateen \& D’Eon, 2008; p. 538). Undergraduate medical education (UME) has been recently reformed in one major domain: integration (Price, 2005; Gonzalo et al., 2017). Curriculum integration, requiring the addition of clinical information and the loss lecture instructional hours, has particularly affected neuroanatomy instruction. Neuroanatomy suffered the largest drop, with an $18 \%$ decrease 
in lecture and laboratory hours in a survey of medical schools $(n=84)$ when converting the curriculum from systems-based to an integrated approach (Drake et al., 2002; Drake et al. 2009). The combined loss of instructional hours and increasing medical student anxiety towards learning neuroanatomy in medical education is an obstacle in need of attention. This study aims to address this challenge by providing a supplemental neuroanatomy instructional tool, for use within an integrated curriculum, designed to reduce medical student neuroanxiety.

Developing a supplemental neuroanatomy eLearning intervention may be an innovative solution as both neuroanatomy instructional hours are decreasing (Drake et al., 2002; Drake et al. 2009) and students are demonstrating increased anxiety towards learning neuroanatomy (Schon et al., 2002; Flanagan et al., 2007; Youssef, 2009; Zinchuk et al., 2010; McCarron et al., 2014; Abulaban et al., 2015). Research studies show medical students perceived neuroanatomy eLearning as beneficial (Foreman et al., 2005; Svirko \& Mellanby, 2017), especially for students preparing for national board examinations (Gould et al., 2008). Most (88\%) students overall found eLearning beneficial with $95 \%$ of students rating eLearning as better than traditional learning tools (Foreman et al., 2005). A 2017 study examined the depth of learning in second year medical students' $(n=869)$ approach to neuroanatomy: deep learning motivated by interest in the subject matter or surface learning motivated by fear of failure (Svirko \& Mellanby, 2017). This study found time spent using eLearning was positively correlated with deep learning; concurrently, a positive correlation between deep learning and academic performance was reported $(r=0.12, p<0.001)$ (Svirko \& Mellanby, 2017). Another study suggests 3D models used in eLearning can improve student learning of 
neuroanatomy when compared to 2D instruction (Estevez et al., 2010). Estevez et al. (2010) found the overall quiz scores were higher for the experimental group receiving the $3 \mathrm{D}$ model $(t[85]=2.02, p<0.05)$ compared to the control group receiving traditional $2 \mathrm{D}$ instruction. In summary, eLearning may decrease overall instructional time, perceived as beneficial and useful for learning neuroanatomy by students, and promotes deep learning and improved student outcomes.

Another important construct to consider when designing an eLearning intervention is academic self-efficacy, as explored in Chapter 4 of this dissertation. Academic selfefficacy has been found to improve overall performance in medical school. Medical students with increased self-efficacy performed better in the first year of medical school in gross anatomy (Burgoon et al., 2012) and on first-year final examinations (StegersJager et al., 2012; Hayat et al., 2020). Medical students with higher self-efficacy scored higher on clinical performance examinations (Mavis, 2001) and self-efficacy predicted a medical student's clinical performance (Opacic, 2003). After graduation, medical residents with higher self-efficacy demonstrated improved patient care experiences (Young et al., 2012). Additionally, there is a complex relationship between academic self-efficacy and academic anxiety. Self-efficacy is based in Social Cognitive Theory (SCT) which theorizes it is the critical mechanism by which one intentionally makes things happen, through taking action, in their environment (Bandura, 2001). SCT suggests the level of one's emotional arousal can impact their self-efficacy (Bandura, 1997). Emotional arousal can improve, or limit, self-efficacy (Bandura, 1997) depending on how one interprets these emotions. If one interprets their internal emotional state (i.e., rapid heart rate, increased blood pressure, increased breathing rate) as excitement, this 
may inspire intentional action towards a goal; however, if one interprets their internal physical and emotional state as threatening or anxiety, it may hinder action and prevent one from taking intentional action. Two educational studies (Nie et al., 2011; Barrows et al., 2013) suggest academic self-efficacy may counteract the effects of educational anxiety. To-date there have been no published studies exploring the relationship between neuroanxiety and neuroanatomy self-efficacy in medical students. One aim of this study is to fill this literature gap by creating a learning intervention- designed to target both improving neuroanatomy self-efficacy and decreasing neuroanxiety- and measuring its effects on both neuroanxiety and neuroanatomy self-efficacy of medical students.

With the shortened curricular hours of an integrated curriculum another important consideration is the development of the self-directed eLearning intervention is a timeefficient design. There is an increasing trend of implementing adaptive eLearning in medical education because adaptive eLearning may decrease instructional time while concurrently increase accuracy and performance (Krasne et al., 2013; Hu et al., 2016; Romito et al., 2016; Samulski et al., 2018). Adaptive eLearning forces students to demonstrate stepwise competencies where students progress through competent areas while additional content is provided for those areas where students struggle. Research shows students tend to focus on subjects in which they are competent and avoid subjects with which they struggle (Brown et al., 2015). Additionally, medical students have demonstrated particularly limited abilities of learning self-assessment (Edwards et al., 2003; Blanch-Hartigan et al., 2011; Hall et al., 2016). If medical students can not accurately assess their own learning, a self-directed (non-adaptive) eLearning intervention may not be as efficient as an adaptive eLearning intervention that forces 
students to demonstrate stepwise competencies and face their weaknesses. Another aim of this study is to compare the effects of a self-directed (non-adaptive) with an adaptive neuroanatomy eLearning intervention on both medical student neuroanxiety and neuroanatomy self-efficacy.

Study Purpose. University of Louisville School of Medicine (ULSOM) recently moved from a systems-based approach towards an integrated curriculum. The integrated first semester, first-year medical student (M1) course-Clinical Anatomy, Development and Examination (CADE) - was developed and implemented in 2014. CADE encompasses anatomy, neuroanatomy, embryology, and relevant clinical examination competencies. CADE is separated into five blocks of material throughout the semester from August to December. One of the challenges is scheduling the vast content to be administered simultaneously with the best integration of the curriculum. As a result of integration, neuroanatomy curricular hours were reduced and information delivery timing changed. Neuroanatomy curriculum was reduced $48.8 \%$ - from 82 hours (4-week systems-based course) to 42 hours (within CADE) to accommodate other first year requirements within the new course. Plans for an efficient, adaptive eLearning module were developed to accommodate the decrease in instructional time.

The purpose of this study is to develop an eLearning intervention designed to both reduce neuroanxiety and increase neuroanatomy self-efficacy. Through a causal comparative design, this study will compare a self-directed (non-adaptive) and an adaptive eLearning intervention on first-year medical student neuroanatomy self-efficacy and neuroanxiety levels. Neuroanxiety will be measured by the scale developed in 
Chapter 3 of this dissertation and neuroanatomy self-efficacy will be measured by scale developed in Chapter 4 of this dissertation. We hypothesize an adaptive eLearning intervention that forces students to demonstrate competency, and face their weaknesses, will decrease neuroanxiety and increase neuroanatomy self-efficacy more than a selfdirected (non-adaptive) eLearning intervention. This study unfolds with an exploration of how self-efficacy and anxiety are related constructs, self-directed learning challenges in medical students, and a review of adaptive eLearning prior to developing and assessing a novel neuroanatomy adaptive eLearning intervention.

\subsection{Background}

\section{Social Cognitive Theory: The Relationship between self-efficacy and anxiety. As}

discussed in Chapter 4 of this dissertation, Social Cognitive Theory (SCT) posits the role one's personal agency plays in an environment (Bandura 2001). Personal agency is the ability to intentionally make things happen by one's actions (Bandura, 2001). Bandura (2001) describes four core features of personal agency: intentionality, forethought, selfreactiveness and self-reflectiveness. People with strong personal agency can adequately assess their abilities (self-reflectiveness), can anticipate future challenges (forethought) while taking action and making course corrections along the way (self-reactiveness) to produce a desired outcome (intentionality). Self-efficacy is considered the critical agentic mechanism (Bandura, 1997) and occupies a pivotal role in SCT (Bandura, 2001). One's self-efficacy beliefs influence the level of challenge one undertakes, their effort, and perseverance when obstacles arise. Bandura (2001) adds that self-efficacy can determine whether failures are motivating or demoralizing through regulation of self-enhancing or 
self-defeating thoughts. In an educational environment, students with high self-efficacy can assess their own strengths and weaknesses (self-reflectiveness), can anticipate challenges on future examinations (forethought) while focusing on strengthening their weaknesses (self-reactiveness) to succeed academically (intentionality). The sources of self-efficacy, therefore, are critical in determining one's personal agency in an educational environment. Bandura (1997) outlined four sources of self-efficacy: Performance, Vicarious Experience, Verbal Persuasion and Emotional Arousal. Below is a description of how the sources emotional arousal in an educational environment, for example medical school, may be related to educational anxiety.

Emotional arousal can improve, or limit, self-efficacy (Bandura, 1997) depending on how these emotions are interpreted. In this way, SCT's self-reflection and one's resulting actions (self-reactiveness) have direct connections to the assessments one makes about their own emotions. A medical student can feel emotional arousal (i.e., increased heart rate, breathing rate, sweating, etc...) and interpret this feeling as excitement which inspires a student to keep studying and learning through intentional action. Alternatively, another medical student may feel the same emotional arousal (i.e., increased heart rate, breathing rate, sweating...) and perceive this emotion as anxiety which demotivates study and preparation. It is in this way that SCT suggests anxiety and self-efficacy are related to each other. One's own self-reflection of their emotional arousal influences their anticipation of future challenges (forethought), actions (self-reactiveness), and the outcomes of those actions (intentionality). 
Self-efficacy's potential moderating effects on educational anxiety. Few studies have discussed the potential of self-efficacy to moderate (decrease) educational anxiety. One study (Barrows et al., 2013) of college students $(n=110)$ measured both student text anxiety and self-efficacy three days prior and immediately after an examination. Bivariate linear regression found self-efficacy did not decrease test anxiety for a single college examination. However, students with higher self-efficacy and lower anxiety scored higher on the examination than those students with lower self-efficacy and higher anxiety. Another study (Nie et al., 2011) found the perceived importance of the task itself may increase student anxiety, especially when student self-efficacy is low. Nie et al. (2011) suggest strengthening a student's self-efficacy beliefs may be a more promising approach than trying to change their perception of the task itself. No published studies todate were found comparing medical student self-efficacy and anxiety in neuroanatomy. One could theorize medical students have a higher self-efficacy than the general population for even taking on the challenge of medical school. Medical students have the documented perception neuroanatomy is an anxiety inducing subject because of its difficulty level (Schon et al., 2002; Flanagan et al., 2007; Youssef, 2009; Zinchuk et al., 2010; McCarron et al., 2014; Abulaban et al., 2015). Especially since the medical school is a high-stakes environment, Nie et al (2011) suggests improving neuroanatomy selfefficacy may more effective than attempting to change student's perception about neuroanatomy, or their neuroanxiety level. This study aims to fill that gap in the literature by measuring the effect of a targeted eLearning intervention on both neuroanxiety and neuroanatomy self-efficacy of medical students. 
SCT and Self-Directed Learning in Medical Students. SCT suggests the ability to assess one's own strengths and weaknesses (self-reflection) and taking action on those weaknesses (self-reactiveness) for a desired outcome (intentionality) can be applied to self-directed learning. A student's ability to assess their own learning and performance (self-reflection) is important because it can influence a student's future study behavior (self-reactiveness) (Brown et al., 2015). However, students tend to focus on subjects in which they are competent and avoid subjects with which they struggle (Brown et al., 2015). Studies show medical students have particularly limited abilities of selfassessment in both learning and performance (Edwards et al., 2003; Blanch-Hartigan et al., 2011; Hall et al., 2016) especially early in their medical school career (Swandon \& Finn, 2004). After one semester of medical school, second-semester medical students were better able to predict their performance on a high stakes gross anatomy practical exam where first-semester medical students lacked that ability (Swandon \& Finn, 2004). A similar trend from a meta-analysis of medical student self-assessment suggests medical students are better able to more accurately predict their abilities later in medical school when compared to early in their medical school career (Blanch-Hartigan et al., 2011). Interestingly, there is a discrepancy between the self-assessment abilities of highachieving and low-achieving medical students. High achieving medical students tend to underestimate their abilities while low-achieving medical students overestimate their abilities (Edwards et al., 2003; Blanch-Hartigan, 2011). One study specific to selfassessment of neuroanatomy learning shows a similar trend. Medical students can not accurately assess their own neuroanatomy knowledge from a standard curriculum, where higher performing students underestimated their abilities and lower performing students 
overestimated their ability (Hall et al., 2016). Creating a self-directed learning intervention for medical students in their first semester of medical school must take into account their inability to accurately predict their learning; especially, early in their medical school career. In light of the Brown et al. (2015) study, first-semester medical students using a self-guided neuroanatomy eLearning intervention may spend more time on the information they already know and avoid the information on which they most need to focus. Alternatively, an eLearning intervention that forces students to demonstrate competency, providing additional content where students demonstrate weaknesses, may differentially improve overall learning of low-performing students. More studies are needed to investigate the differential effect of adaptive eLearning on medical students of varying abilities.

Review of Adaptive eLearning in Medical education. Adaptive learning is a teaching technique that forces students to demonstrate stepwise competencies; students quickly progress through competent areas while additional time and/or content are provided for those areas in which an individual student struggles. Adaptive eLearning applied to medical education shows students decrease learning time while concurrently increasing accuracy and overall performance (Krasne et al., 2013; Hu et al., 2016; Romito et al., 2016; Samulski et al., 2018). Adaptive eLearning modules are being innovated in medical education in the fields of surgical education (Hu et al., 2016) histopathology (Krasne et al., 2013), anesthesiology (Romito et al., 2016), and cytopathology (Samulski et al., 2018). A review of adaptive eLearning in medical education was conducted prior to developing a novel adaptive eLearning neuroanatomy intervention. 
The adaptive teaching method was shown to be more time effective in a surgical education study by $\mathrm{Hu}$ et al. (2016). In a randomized study of first- and second-year medical students, one group of 24 students received the adaptive method of using a surgical simulator while the control group received the traditional time spent with the simulator technique. The adaptive simulator assigned a numerical score for each attempt (based on predetermined minimum checklist criteria) and recorded the elapsed time for each attempt of three different surgical techniques. If a participant that once passed a task by obtaining the minimum score then later failed that same task, this was designated as a relapse. The control group participants were graded on the same checklist criteria by a teaching assistant and elapsed time was recorded for each attempt. A post-exam for both groups with a blinded, experienced surgical faculty member was used to measure technical proficiency on the surgical simulator. Three attempts were allowed for each task and the top two scores for each task were averaged and normalized. There was no statistically significant difference in the post-test between the two groups: (1) the adaptive learning group averaged $92.1 \%$ with +16.2 improvement from baseline on their post-test; while (2) the control group demonstrated a $93.5 \%$ average on their post-test and +15.7 improvement (Hu et al., 2016). However, the adaptive learning surgical simulator individualizes the training to each participant's learning patterns and seems to be more time effective. It provides an advantage at detecting relapses as it monitors in real time. Since the adaptive learning simulator seeks to correct deficiencies during the task and requires the learner to become proficient before advancing, it provides more guidance to allocating time where one needs improvement. All participants in the adaptive learning group obtained proficiency in all three surgical techniques during 6 (1-hour) sessions or 
less. Since only $29 \%$ of the control group obtained proficiency in all three techniques within 7 (1-hour) sessions, without guidance, the participants did not allocate their time appropriately on their own to correct their deficiencies. The adaptive learning approach both decreases learning time and improves accuracy and performance.

In a single group experimental pilot study, an adaptive eLearning module for firstyear medical students to teach introductory histopathology demonstrates increased accuracy and retention Krasne et al. (2013). A recurring theme in medicine is the ability of a physician to recognize patterns. It has been historically difficult to teach pattern recognition in traditional classroom setting. This adaptive eLearning module was designed to improve histopathological pattern recognition of four skin conditions: (1) normal; (2) inflammation; (3) cell injury; and (4) neoplasia. Eight total categories were tested, one at low resolution (x4-10) and one at high resolution (x20-40) for each of the four categories. For each category question, the target response time was set to 12 seconds and the student was timed-out of the question at 24 seconds. Feedback was given to the learner for each response whether it was a correct or incorrect answer. Once a learner performed accurately for each category, within the target response time, three times, the concept was considered fluent, or "learned", and that category was no longer tested. First-year medical students $(n=161)$ in 2011 and in $2012(n=155)$ utilized the adaptive eLearning module and completed a pre-test. A year later, the same medical students were post-tested to assess memory retention. Three different forms of the test were randomly assigned to account for any possible difference in difficulty level between the exams. This study demonstrated increased retention relative to the pre-test values, with $p<0.0001$ and an effect size (Cohen's $d$ ) of 0.8 , when comparing the delayed 
post-test (one year later) with those of the pre-test (Krasne et al., 2013). This suggests a significant increase in both accuracy and retention. One limitation of this study is the lack of a control group for comparison. Another limitation is the author of this study created the proprietary software for the adaptive eLearning module and the significance of the findings are to be taken with caution.

Another, more rigorous randomized study, investigated learning outcome differences of interpreting transesophageal echocardiography (TOE) between two groups of anesthesiology residents (Romito et al., 2016). An experimental group utilizing an adapting eLearning module was compared to a control group of residents only attending a traditional lecture. As in histopathology pattern recognition, TOE pattern recognition is also a difficult concept to learn quickly and an adaptive eLearning module was created and tested to determine its efficacy. Similar to the previous study, each diagnostic category was tested and 3 consecutive correct answers within a target response time was needed to achieve fluency. Two versions of the tests were created to minimize the instrumentation threat of differences in exam question difficulty to internal validity. Twelve residents were randomly assigned to the adaptive eLearning module group and 12 residents to the control group (no adaptive eLearning module). All groups received a lecture on TOE, a pre-test measuring accuracy and response time. The adaptive eLearning module group completed the post-test immediately after and both groups completed a post-test 6 months later. The adaptive eLearning module group had a significant improvement in accuracy and fluency (79\% versus $70 \%)$ in correctly diagnosing cardiac pathology. After 6 months, the adaptive eLearning module group remained significantly higher than the control group performance over this period 
(Romito et al., 2016). An adaptive eLearning module can significantly improve accuracy and fluency in diagnosing cardiac pathology by TOE, in a group of anesthesiology residents compared with residents not completing the adaptive eLearning module. The adaptive eLearning module allows students to focus on the topics where they need more time and focused attention.

A randomized, mixed-methods study designed to assess adaptive eLearning compared to traditional study methods in cytopathology showed adaptive eLearning not only improved test scores, but it was also well received by medical students (Samulski et al., 2018). Medical students perceived adaptive eLearning as an efficient way to review the material. They described the adaptive eLearning module as more engaging than lectures and textbooks. Medical students favored the immediate feedback and interactivity of the module; however, the inability to review prior content within the module was described as a limitation of the adaptive module. Overall, these studies suggest adaptive eLearning increases medical student outcomes and retention, while concurrently decreasing learning time. Adaptive eLearning was well received by medical students as a supplemental learning tool. This research guided our decision to develop and assess an adaptive neuroanatomy eLearning intervention designed to both reduce neuroanxiety and increase neuroanatomy self-efficacy for first-year medical students.

\subsection{Methods}

Study Population. A convenience sample of first-year medical students enrolled at a large, public, Southeastern university were invited to participate in the online eLearning modules and assessment surveys administered in Qualtrics ${ }^{\circledR}$. One cohort of first-year 
medical students were invited to participate in the non-adaptive eLearning intervention and surveys in the Fall 2018 semester $(n=160)$. A second cohort of first-year medical students were invited to participate in the adaptive eLearning intervention and assessment surveys in the Fall 2019 semester $(n=162)$.

Data Collection Procedures. The director of the integrated first semester medical school course sent an email invitation to participate in the study during the Fall 2018 and Fall 2019 semesters. First-year medical students were invited to complete the NAS survey capturing neuroanxiety levels at the beginning of the semester (pre-course) and again at the end of the semester (post-course). First-year medical student neuroanxiety levels were measured using the NAS scale developed in Chapter 3 of this dissertation. Four weeks following the survey, students in the Fall 2018 and Fall 2019 first-semester integrated medical school course were provided access to their respective non-adaptive and adaptive eLearning modules during the semester. The modules were published in a Learning Management System (LMS) format from Articulate Storyline ${ }^{\circledR}$ and subsequently uploaded to RedMed ${ }^{\circledR}$ for student access. RedMed ${ }^{\circledR}$ provides the number of distinct users accessing the module and the time the module was accessed; module usage data will be reported.

An optional link to the NSES surveys, capturing neuroanatomy self-efficacy, in Qualtrics ${ }^{\circledR}$ was provided to access the online pre-module and post-module surveys before and after using the eLearning module. Neuroanatomy self-efficacy of first-year medical students was measured by NSES scale developed in Chapter 4 of this dissertation. Students were made aware their data would be anonymized and used for this study if they 
chose to participate in the survey. Completed surveys were exported from Qualtrics into a csv file in Microsoft Excel ${ }^{\circledR}$. IP addresses were deleted from the files to protect anonymity. The files were imported into IBM SPSS ${ }^{\circledR}$ Version 26 where missing or noncomplete surveys were deleted prior to analysis.

Neuroanxiety Scale (NAS). The 7-item NAS scale (Appendix B) was developed in Chapter 3 of this dissertation. Respondents were asked to subjectively rate how often they were bothered by seven anxiety symptoms during the past two weeks while studying neuroanatomy, during neuroanatomy lecture or while performing a neurological exam $(0=$ Not at All, 1= Several Days, 2= More Than Half the Days and 3= Nearly Every Day $)$. These items encompass the scope of the operational definition of neuroanxiety: fear of neuroanatomy, ability to learn neuroanatomy and its clinical applications. Scoring of the NAS-7, adapted from the GAD-7 (Spitzer et al., 2006), is reported as a sum total of raw scores where significantly higher scores represent greater self-reported neuroanxiety.

Neuroanatomy Self-Efficacy Scale (NSES). The 13-item NSES scale (Appendix B) was developed in Chapter 4 of this dissertation. Respondents were asked to rate their confidence level on a 5-point Likert scale in doing 13 different neuroanatomy specific tasks (1= Not at All Confident, 2= Only a Little Confident, 3= Fairly Confident, 4= Very Confident and 5= Totally Confident). Neuroanatomy self-efficacy is scored as raw scores reported as a sum score of all items where lower scores signify lower self-rated neuroanatomy self-efficacy while higher scores represent greater self-reported 
neuroanatomy self-efficacy. For this scale, higher scores are indicative of higher neuroanatomy self-efficacy.

Module development. Development of both the non-adaptive and adaptive eLearning modules are described below. The content selection, features designed to reduce neuroanxiety, and features designed to increase neuroanatomy self-efficacy consistent in both modules will be explained first. Next, applying adaptive eLearning to the module, with specific features only applicable to the adaptive module, will be described. A flow chart of the non-adaptive and adaptive modules can be found in Figures 5.1 and 5.2, respectively. Both modules allow students to progress at their own pace; however, the non-adaptive module allows for bidirectional progression through the module while the adaptive module forces forward progression to allow for the adaptive capabilities.

Content selection. Three major content areas for the eLearning modules were selected based on a number of sources: student course evaluations, undergraduate medical education office evaluations, and neuroanatomy education research (Flanagan et al, 2007; Youssef, 2009; Zinchuk et al., 2010; Abulaban et al., 2015; Javaid et al., 2018). Three selected evidence-based content themes were consistent among sources: tracts, brainstem anatomy and case based clinical lesion problems. It was decided to focus the eLearning intervention on the three major tracts: dorsal column medial lemniscus (DCML), corticospinal tract (CST), and the spinothalamic tract (STT). First-year medical students must master these pathways prior to tackling more detailed brainstem anatomy and understanding how to solve case based clinical lesion problems. 
Features designed to reduce neuroanxiety. Specific module features were incorporated within the content, instruction, communication, and organization domains informed by the qualitative study in Chapter 2 of this dissertation. Figure 5.3 shows the content features of the module: neuroanatomy vocabulary assistance and $3 D$ relationships. Optional neuroanatomy vocabulary assistance is provided to students (Figure 5.3A and 5.3B) as complex jargon and lexicon were described by students as making neuroanatomy unnecessarily difficult. $3 D$ relationships in cross-sectional anatomy (Figure 5.3C and 5.3D) are provided along with the pathway information to provide students assistance with orienting where these three-dimensional neuroanatomical structures are located on a two-dimensional image. The instruction domain features of the module are shown in Figure 4: step-by-step instruction, clinical context, and structurefunction correlation. Students are not shown the pathway in its entirety at first, students are alternatively guided through step-by-step instruction (Figure 5.4A and 5.4B) by clicking on each step of the pathway in a progressive manner. Clinical context is provided with optional spinal cord lesion cases describing their clinical symptoms (Figure 5.4C and 5.4D). Simple structure-function correlation is shown through an example of how each pathway may be used in everyday life (Figure 5.4E and 5.4F). Overestimation of student knowledge was the main communication obstacle identified in our students. Figure 5.5 shows the communication content which provides students optional descriptions and definitions of commonly used neuroanatomy jargon. These optional definitions (Figures 5.5A- 5.5D) are provided to reduce the embarrassment and anxiety students may feel asking these questions in another format (i.e., lecture). Figure 
5.6 shows the consistent organization of the module. The main menu (Figure 5.6A) contains an icon to allow the student to enter each pathway. Each pathway unfolds following the same order to provide organizational consistency. Additionally, the module is organized so students may progress at their own pace (Figure 5.6B); students control slide advancement by clicking on the buttons designed to reduce their anxiety while learning neuroanatomy.

Features designed to increase neuroanatomy self-efficacy. Module items designed to improve neuroanatomy self-efficacy are based on Social Cognitive Theory (Bandura, 1986, 2001). Sources of self-efficacy (performance, vicarious experience, verbal persuasion and emotional arousal) described by Bandura (1986) can be applied to neuroanatomy experiences to improve neuroanatomy self-efficacy. Both the non-adaptive and adaptive modules contain features to improve the performance, verbal persuasion, and emotional arousal aspects of neuroanatomy self-efficacy. (The adaptive module contains additional vicarious experiences that will be described in the development of the adaptive module.) Performance mastery is provided within the module through successful completion of neuroanatomy questions in increasing level of difficulty. Early on in the pathways, simple questions are asked with answers and explanations provided. Intentionally increasing the level of difficulty, providing feedback with explanations of incorrect answers, is intended to improve neuroanatomy self-efficacy through performance. The module is also designed to intentionally reduce emotional arousal (Figure 5.7). Soft-feedback in the form of Oops instead of wrong or incorrect is provided to students when they receive wrong answers. The Let's Explain section provides 
students with detailed explanations of incorrect responses as an instructional tool (Figure 5.7A). Additionally, the module contains characters speaking to the students (Figure 5.7B). The characters act as guides and are designed to provide verbal persuasion at key points within the module. Verbal persuasion is provided by quotes from the characters within the module (Figure 5.8A and 5.8B) and describe hints on how best to study for neuroanatomy in the introduction. These quotes from the characters are summarizations of real quotes from actual second-year medical students to first-year medical students as captured in the piloting of the module. Inclusive characters representing students from different race, socioeconomic status, gender, and age were included within the module. These characters acknowledge difficult questions throughout the module and persuade the student to push on towards successful completion. Successful completion of an acknowledged difficult neuroanatomy problem was designed to improve student neuroanatomy self-efficacy.

eLearning Module Structure. Both non-adaptive and adaptive modules were designed in the Articulate Storyline ${ }^{\circledR}$ platform with four sections. An introduction section reviews pathway basics, nomenclature and their functions prior to granting the user access to the main menu (see Figures 5.1 and 5.2 for the non-adaptive and adaptive module design layout, respectively). Subsequently, a main menu grants the user access to three other sections, each explaining a major neuroanatomy pathway: dorsal column medial lemniscus (DCML), spinothalamic tract (STT), and the corticospinal tract (STT). The non-adaptive module allows the student bidirectional access to advance or go back and 
review previous content (represented by the bidirectional arrows in Figure 5.1). There is no time limit for the completion of either module.

Creating the adaptive module required designing novel checkpoints (to assess competency) and remediation loops (to provide additional content and further competency assessment). Below a description of applying adaptive eLearning to neuroanatomy in both the utility and mechanics of the adaptive eLearning module (Figure 5.2).

\section{Applying adaptive eLearning to neuroanatomy}

Adaptive features: Utility. As just described, the novel adaptive content was created using checkpoints and remediation loops. Each checkpoint consists of a short quiz (3-5 questions) ensuring competency before module progression. The student user must obtain $100 \%$ competency on one attempt of all the questions in the checkpoint to progress within the module. One hundred percent competency was chosen to maintain brevity to ensure the user had sufficient time to complete all aspects of the module, including all potential remediation loop content, within the allotted timeframe (two hours). This also ensures only students with the ability to apply their knowledge may skip the adaptive content and progress within the module. If the student passes the checkpoint with $100 \%$ competency

on the first attempt, they progress through to the next section of the module. If the student fails a checkpoint, they are shown remediation loop content. The user is not explicitly told they have failed or that the supplemental content is considered remediation. A remediation loop contains supplemental content that reviews the information and 
provides more comprehensive explanations. After reviewing this supplemental content, the user is asked to take a remediation quiz (similarly, the user is not explicitly told this is a remediation quiz). The remediation quiz contains questions similar to (although not identical to reduce question bias by allowing students to memorize questions and answer choices) the checkpoint questions and the user may continue once they obtain 100\% competency. The user is allowed to take the remediation quiz and review incorrect answers, with explanations, as many times as required to obtain $100 \%$ competency. Once the remediation quiz is successfully completed, the user is taken back to the main content of the module. The student is now back on track to receive the content they would have received if they initially passed the checkpoint.

The checkpoints within each pathway were administered at the same time point within the module to ensure content consistency. Each tract had two checkpoints: Somas \& Synapses and Tract Anatomy in 3D. The Somas \& Synapses checkpoint (Figure 5.9) assessed a student's knowledge of the location of neuronal cell bodies, synapses, axons and decussation points within each pathway (The DCML pathway was divided into gracilis and cuneatus pathways and, as such, each contain a Somas \& Synapses checkpoint as shown in Figure 5.2). Competency on the Somas \& Synapses checkpoint (Figure 5.9B), administered after the completion of the pathway anatomy content, results in the user advancing on to the pathways review. If the user does not demonstrate $100 \%$ competency, they are brought to a Drawing Video remediation loop (Figure 5.9C). The Drawing Video remediation demonstrates how to draw the pathway on paper along with an audio description of the location of the cell bodies, axons, synapses, and decussation points within the tract. The student is encouraged to draw along with the video. This 
video models how to draw the tracts quickly, for an example during an examination, and was intended to increase neuroanatomy self-efficacy through vicarious experience (Bandura 1986). Subsequently, a Drawing Video remediation quiz asks similar (not identical) questions about the location of the neuronal cell bodies, synapses, axons and decussation points within the pathway. The student is allowed to return to pathway review once competency on the Drawing Video remediation quiz is acquired.

A pathways review shows the student the location of the neurons within the pathway on spinal cord and brainstem cross-sections. The Tract Anatomy in 3D checkpoint (Figure 5.10) assesses a student's ability to identify the location of neuronal cell bodies, axons, synapses, and decussation points on brainstem and spinal cord crosssections (Figure 5.10A). If the student user does not demonstrate 100\% competency on the Tract Anatomy in 3D checkpoint (Figure 5.10B) on the first attempt, the user is brought to a 3D Model Video remediation loop (Figure 5.10C). A 3D model was incorporated into the module from the results of Estevez et al. (2010) showing improved medical student learning outcomes using 3D models. The 3D paper model used, courtesy of Nicole Herring Ph.D., shows the cross-sectional anatomy of the spinal cord along with caudal and rostral cross sections of the medulla, pons, and midbrain. The printed crosssections contain anatomical landmarks associated with the three major tracts (dorsal column medial lemniscus, spinothalamic, and corticospinal) on thick, cardstock paper. A hole punch was used to allow for colored pipe cleaners to be threaded through the crosssections demonstrating the anatomical location of the axons and cell bodies within the pathway in a three-dimensional space. A video showing this model, along with an audio description of the neurons within the pathway, is provided. The user has the ability to 
stop, pause and replay the video as many times as desired. After completion of the video, the user is asked similar (not identical) questions on a 3D Model Video remediation quiz. Once $100 \%$ competency on the $3 D$ Model Video remediation quiz is obtained the user is brough to a congratulations slide to demonstrate competency and completion of the pathway.

Adaptive features: Mechanics. The Articulate Storyline ${ }^{\circledR}$ platform was chosen for ease of creating adaptive features and web-publishing capabilities with minimal knowledge of computer coding needed to create the functionality. Details on how to create the checkpoint and remediation loop competencies are described below.

Checkpoint competency was created using the multiple-choice quiz function. One attempt per question and a passing score of $100 \%$ were selected in the quiz function menu in Articulate Storyline ${ }^{\circledR}$. A quiz review slide was shown to the user upon quiz completion. The pass or fail quiz review slide shown, depending on the student's quiz score, determines the next step within the module. The pass page contains a button labelled continue; a trigger is added to the pass continue button to advance the user to the next slide in the main section of the module. Alternate ways of advancing the slides in a forward or backward direction were disabled by removing the "next" and "prev" button and disabling the user the ability to advances the slides without demonstrating competency. The fail quiz review slide also contains a button labelled continue; a trigger is added to the fail continue button to advance the user to the first slide in the remediation loop. 
Remediation loop competency was also created using the multiple-choice quiz function. Students are asked to demonstrate remediation loop competency by asking similar, not identical multiple-choice questions, to prevent question bias by allowing students to memorize the questions. The questions within the Articulate Storyline ${ }^{\circledR}$ question bank allows randomization of both remediation quiz questions and answer choices. Unlimited attempts were selected (with a passing score of 100\%) in the quiz function menu to allow the user to demonstrate competency before advancing in the module. Explanations of incorrect answers were provided by including variables with an if/then statement and revealing a hidden layer. A variable for each incorrect answer choice, with an if/then statement, that shows a hidden layer when that specific incorrect choice was selected. This hidden layer revels an explanation of why that answer choice was not correct and contains a hint for the student (Figure 5.11). A variable and hidden layer was inserted into the module for each incorrect answer choice in the remediation loop quiz questions. Similarly, these quiz questions were only allowed to advance in a forward direction by disabling any alternative slide advancement.

Pilot. A pilot of 5 second-year medical student volunteers was conducted. Second-year medical students were asked for feedback assessing module design and aesthetics, ease of module use, and the functionality of the link to Qualtrics ${ }^{\circledR}$ survey from the module. Second-year medical students were also asked for helpful hints to studying for and learning medical neuroanatomy. These suggestions were provided to the first-year medical students throughout the module for increasing neuroanatomy self-efficacy by verbal persuasion. The design of the module, particularly with the characters, were well 
received by the second-year medical students. Non-functioning module triggers were reported to the author and subsequently repaired. The average time for a second-year medical student to complete the module was 25 minutes.

Data Analysis. A causal comparative study was used to determine whether an adaptive neuroanatomy eLearning intervention increases neuroanatomy self-efficacy (NSE) and decreases neuroanxiety (NA) more than a non-adaptive intervention. A chi square test of gender and independent $t$-tests of GPA and MCAT scores between the Fall 2018 (nonadaptive) and Fall 2019 (adaptive) was conducted to ensure equivalent study populations for this causal comparative study.

An independent samples $t$-test was performed on the pre-test NSE scores between the control (Fall 2018) and experimental (Fall 2019) groups. The pre-test NSE scores differed significantly, therefore an analysis of covariance (ANCOVA) was used to determine differences in the pre- and post-test NSE scores while controlling for the differences in the pre-test scores. Our hypothesis is that the adaptive learning intervention will increase NSE scores more than the non-adaptive learning intervention, after controlling for differences in pre-test scores. The significance level used for hypothesis testing will be held at $p<0.05$.

An independent samples $t$-test was performed on the pre-test NA scores between the control (Fall 2018) and experimental (Fall 2019) groups and no significant difference between the means was found. A mixed design analysis of variance (ANOVA) was used to determine differences in the pre- and post-test NA scores since the pre-test NA scores did not differ significantly. Our hypothesis is that the adaptive learning intervention will 
decrease NA scores more than the non-adaptive learning intervention. The significance level used for hypothesis testing will be held at $p<0.05$.

\subsection{Results}

5.4.1 Module Usage Data and Survey Response Rates. The Fall 2018 semester reported 154 distinct student users of the non-adaptive module. This is a $96.3 \%$ utilization rate with 160 first-year medical students enrolled in the Fall 2018 semester. The Fall 2019 semester showed 162 distinct student users of the adaptive module. This is a 100\% utilization rate with 162 students enrolled in the Fall 2019 semester.

Pre- and post-module neuroanatomy self-efficacy (NSES) survey response rates are lower than module usage. The Fall 2018 semester found a 13.75\% response rate; $n=22$ students completed the pre-module neuroanatomy self-efficacy (NSES) survey and $n=15$ students completing both the pre-module and the post-module NSES surveys. The Fall 2019 semester observed a 38.9\% response rate; $n=115$ students completed the premodule NSES survey with $n=63$ students completing both the pre-module and the postmodule NSES surveys.

Pre- and post-course neuroanxiety (NAS) surveys reported response rates even lower than the pre- and post-module NSES survey response rates. The Fall 2018 semester found a $13.1 \%$ response rate; $n=25$ students completed the pre-course neuroanxiety (NAS) survey and only $n=21$ students completed both the pre- and post-course NAS surveys for matched data samples. The Fall 2019 semester observed a 16\% response rate; $n=99$ students completed the pre-course NAS survey with only $n=26$ students completing both the pre-and post-course NAS surveys providing matched data samples. 
5.4.2 Descriptive Statistics. Chi square and independent $t$-tests were used to ensure equivalent groups for this causal comparative study. A chi square test indicated there were no significant gender differences between first-year medical students in the nonadaptive-Fall 2018 and the adaptive-Fall $2019\left(X^{2}[1, n=322]=0.02, p=0.88\right)$ semesters. Independent $\mathrm{t}$-tests showed there were no significant differences in GPA, $t(320)=1.35$, $p=0.18$, and MCAT scores, $t(320)=1.79, p=0.07$, between first-year medical students in the non-adaptive-Fall 2018 and adaptive-Fall 2019 semesters.

The non-adaptive module-Fall 2018 participants reported higher average premodule neuroanatomy self-efficacy (NSES) scores, $M=16.45, S D=4.83$, than the adaptive-Fall 2019 participants, $M=11.26, S D=4.84$. The results of the independent samples $t$-test show a significant difference in pre-test NSES scores between nonadaptive and adaptive groups, $t(135)=4.62, p<0.001$, with a large effect size (Cohen's $d=1.07)$.

Descriptive statistics showed non-adaptive module-Fall 2018 participants selfreported lower pre-course neuroanxiety levels, $M=3.43, S D=4.57$, than adaptive participants, $M=5.27, S D=5.4$, however this difference was not significant, $t(45)=1.24$, $p=0.22$.

\subsubsection{Neuroanatomy self-efficacy (NSES) differences using a non-adaptive compared} to an adaptive neuroanatomy eLearning intervention. An analysis of covariance (ANCOVA) was used to find differences in the pre-module and post-module NSES scores when comparing non-adaptive and adaptive eLearning intervention groups while 
controlling for the differences in the pre-module NSES scores. A Shapiro-Wilk test showed no significant departure from normality for both groups $(W(15)=0.95, \mathrm{p}=0.58$ and $W(63)=0.88, p=0.05)$. These data met the homogeneity of variances assumption $(F$ $=1.52, p=0.22)$ for an ANCOVA.

The results of the ANCOVA showed a significant effect for module type on posttest neuroanatomy self-efficacy scores (NSES) after controlling for pre-test neuroanatomy self-efficacy scores, $F(1,75)=6.2, p=0.015$ (Table 5.1). The reported partial eta squared effect size $\left(\eta^{2}=0.076\right)$ suggested a moderate practical significance. First-year medical student participants in the Fall 2019 semester reported significantly higher post-module NSES scores after using the adaptive intervention compared to the Fall 2018 first-year medical school participants using the non-adaptive intervention (Figure 5.12). These findings support our hypothesis that an adaptive eLearning intervention increases neuroanatomy self-efficacy scores more than a non-adaptive eLearning intervention, while controlling for the differences in the pre-module NSES scores.

\subsubsection{Neuroanxiety differences using a non-adaptive compared to an adaptive} neuroanatomy eLearning intervention during the course of the semester. A mixed design analysis of variance (ANOVA) was performed to determine the differences in the pre-course and post-course neuroanxiety (NAS) scores between participants using the non-adaptive compared to the adaptive eLearning intervention. The results of the mixed design ANOVA showed that there was no significant effect of module type $(F(1,45)=$ 2.16, $\left.p=0.148, \eta^{2}=0.046\right)$ on neuroanxiety overall (Table 5.2). The non-adaptive 
participants began the Fall 2018 semester with pre-course NAS scores $(M=3.42$, $S D=4.57, n=21)$; these scores were slightly lower at the end of the Fall 2018 semester $(M=3.19, S D=3.98, n=21)$ (Figure 5.12). The adaptive participants began the Fall 2019 semester with higher pre-course NAS scores $(M=5.27, S D=5.40, n=26)$. However, these scores were not significantly lower for these participants at the end of the Fall 2019 semester after having used the adaptive eLearning intervention during the course of the semester $(M=4.69, S D=4.29, n=26)$ (Figure 5.13). Both non-adaptive and adaptive participants showed a decrease in neuroanxiety levels at the end of the first-semester of medical school. However, the adaptive module did not significantly decrease post-course neuroanxiety levels of first-year medical students compared to the non-adaptive module.

5.5 Discussion. Our findings show that an adaptive neuroanatomy eLearning intervention, designed to target both neuroanatomy self-efficacy and neuroanxiety, improved neuroanatomy self-efficacy but did not significantly reduce neuroanxiety in our medical student population. In this causal comparative study, the Fall 2018 first-year medical students were administered the self-directed (non-adaptive) module. The Fall 2019 first-year medical students were given the adaptive module and the differences in neuroanatomy self-efficacy and neuroanxiety before and after using the module for both groups were recorded. Chi square analyses and independent $t$-tests demonstrated both Fall 2018 and Fall 2019 incoming first-year medical students were equivalent with respect to binary gender, GPA and MCAT scores.

First-year medical students using an adaptive eLearning intervention showed an increase in neuroanatomy self-efficacy (NSES) compared to students using a 
self-directed (non-adaptive) eLearning intervention, while controlling for the differences in the pre-module NSES scores. These results suggest demonstrating competency in the adaptive module revealed to students their strengths and weaknesses. As suggested by studies showing medical students have limited self-assessment capabilities (Edwards et al., 2003; Blanch-Hartigan et al., 2011; Hall et al., 2016), especially in their first year (Swandon \& Finn, 2004), they may need assistance with identifying academic weaknesses. The adaptive module highlighted student's weaknesses through required competency-based questions. This forced competency may have been particularly helpful for lower performing medical students, who tend to overestimate their abilities (Edwards et al., 2003; Blanch-Hartigan, 2011). Revealing students strengths and weaknesses allows them to focus on their weaknesses, instead of their inclination to avoid weaknesses and focus study time on their strengths (Brown et al., 2015).

These results also suggest the sources of neuroanatomy self-efficacy are consistent with the sources of self-efficacy as described by Bandura (1977). The module increased neuroanatomy self-efficacy through performance mastery by having students answering direct questions and completing lesion isolation problems. The module improved neuroanatomy self-efficacy through vicarious experience by watching videos of modeling the thought process of how to solve clinical lesion problems. Emotional arousal was kept low throughout the module by providing students guided feedback to incorrect answers and not using the traditional symbols and colors for wrong answers (i.e. wrong, an $X$, or variations of red colors). Verbal persuasion, used minimally, provided guidance and support as students completed the module. 
Neuroanxiety in our study was slightly decreased on both studies with utilization of both modules. The module features of content, instruction, communication, and organization informed from our qualitative study in Chapter 2 of this dissertation showed promising results with decreasing neuroanxiety. The modules incorporated features, such as, consistent organization, a step-by-step instructional approach, vocabulary assistance, 2D to 3D orientation, and clinical correlations to ease neuroanxiety. Our results, even though not significant, showed some effect on reducing medical student neuroanxiety.

However, there are some limitations to the results of this study. While module utilization was high, few students completed all surveys which limited our sample size and may contributed to non-response bias. For example, a larger survey response would give a more accurate representation of the student population completing the module. It is unknown only the high performing students, perhaps with high neuroanatomy selfefficacy and concurrent low neuroanxiety, took the extra time to complete the surveys. Additionally, there were some differences in design between the adaptive and nonadaptive modules. In the adaptive module only, select additional remedial content contained vicarious experiences in the form of pre-recorded videos. These videos modeled how to draw the tracts and walked through the thought process of solving a clinical lesion problem. If students used these videos in the remedial loop and found them helpful for learning tract information, these vicarious experience videos may have biased the data by increasing neuroanatomy self-efficacy scores among those in the adaptive learning group only. The utilization data provided by RedMed ${ }^{\circledR}$ did not include how many times students accessed these vicarious experience videos by the adaptive module users. Nonetheless, vicarious experience is not as important as performance mastery 
when considering the sources of self-efficacy according to Bandura (2001). Module design features based on performance mastery, the most vital source of self-efficacy (Bandura, 2001), were held constant between the non-adaptive and adaptive modules. These findings support our hypothesis that an adaptive eLearning intervention increases neuroanatomy self-efficacy scores more than a non-adaptive eLearning intervention.

Using an adaptive eLearning intervention did not show a significant decrease in neuroanxiety compared to students using a self-directed (non-adaptive) eLearning intervention. One major limitation of this study was that survey response rates were much lower than module utilization. A larger response rate to the surveys and therefore a larger sample size, encompassing more of the student population, would reduce the potential for survey non-response bias. A larger sample size would also increase the statistical power by increasing the probability of detecting a difference between the mean neuroanxiety scores. Since no difference in mean neuroanxiety scores was found, a larger sample size may reduce the probability of this being a Type II error. Additionally, the differential timing of survey administration may have unintentionally skewed the results in favor of the module's effectiveness for neuroanatomy self-efficacy. As described in the methods, the neuroanxiety scales were administered at the beginning and the end of the semester (pre- and post-course) while the neuroanatomy self-efficacy surveys were administered immediately before and after the module (pre- and post-module). The differential timing of survey administration was intended to prevent survey fatigue while simultaneously collecting data for all aspects of this dissertation project. However, maturation, or students learning neuroanatomy content throughout the semester, may have resulted in decreased neuroanxiety post-course scores as a function of learning neuroanatomy over 
time. Therefore, this may have unintentionally skewed the results in favor of finding a larger mean difference for neuroanatomy self-efficacy before and after utilization of the module.

These initial data are consistent with the conclusion by the Nie et al (2011) study. This study suggests that strengthening a student's self-efficacy beliefs may be a more promising approach to moderating academic anxiety rather than trying to change their perception of the (high-stakes) task itself. Medical neuroanatomy is a high-stakes environment which may induce neuroanxiety, and even perhaps, general anxiety or other anxiety disorder in predisposed medical students. Our findings suggest an adaptive eLearning intervention significantly improved neuroanatomy self-efficacy. Perhaps one route to creating alternative educational interventions to address neuroanxiety (previously called neurophobia) may be to target neuroanatomy self-efficacy instead of trying to reduce neuroanxiety or change a medical student's perception that neuroanatomy is difficult.

One limitation of this study was in the causal comparative design. We were unable to conduct an experiment with random assignment, for example randomly assigning half of the class the adaptive module and the other half a non-adaptive module. Our medical students study in close-knit groups and frequently share study materials. It was likely students in the adaptive group would share their content with the non-adaptive group and threaten the validity of our study. Another limitation of this study was the inability of RedMed ${ }^{\circledR}$ to capture individual session utilization data. Our intention was to capture module session usage time per user, to assess any differences in how long students used the adaptive compared to the non-adaptive modules. However, the time 
usage data reported a running total for each user and on visual inspection of the module time usage data found many users accessed the module for over 36 hours at one time. This may suggest if the module was left open on the computer screen, it kept recording time usage data although the student was no longer actively working in the module. Alternatively, this could suggest a student logged into the module repeatedly and completed it multiple times for a total of 36 hours. The decision was made to not report these data in the analyses because the long module usage times were unable to be resolved.

In summary, an adaptive neuroanatomy eLearning intervention increased firstyear medical student neuroanatomy self-efficacy. This novel study adds to the literature by showing the utility of applying adaptive eLearning to medical neuroanatomy. The adaptive module slightly decreased neuroanxiety, albeit not significantly as anticipated, but it's benefit to neuroanxiety may be indirect. Given the moderating effect of selfefficacy on academic anxiety (Nie et al., 2011; Barrows et al., 2013) perhaps future educational interventions to address neuroanxiety (also called neurophobia) could instead focus on improving neuroanatomy self-efficacy to indirectly reduce neuroanxiety. Adaptive eLearning may be a time efficient way to incorporate a supplemental learning tool within an integrated curriculum. Students responded favorably to the learning intervention through course evaluations. This novel study supports future investigation into creating eLearning tools targeting academic self-efficacy to moderate academic anxiety. These findings may suggest an idea that is generalizable toward helping medical students alleviate academic anxiety in other areas of medical education. 
Acknowledgements. The author wishes to acknowledge Dr. Nicole Herring for creating the 3D paper tract model used for this project. 


\section{FIGURE 5.1}

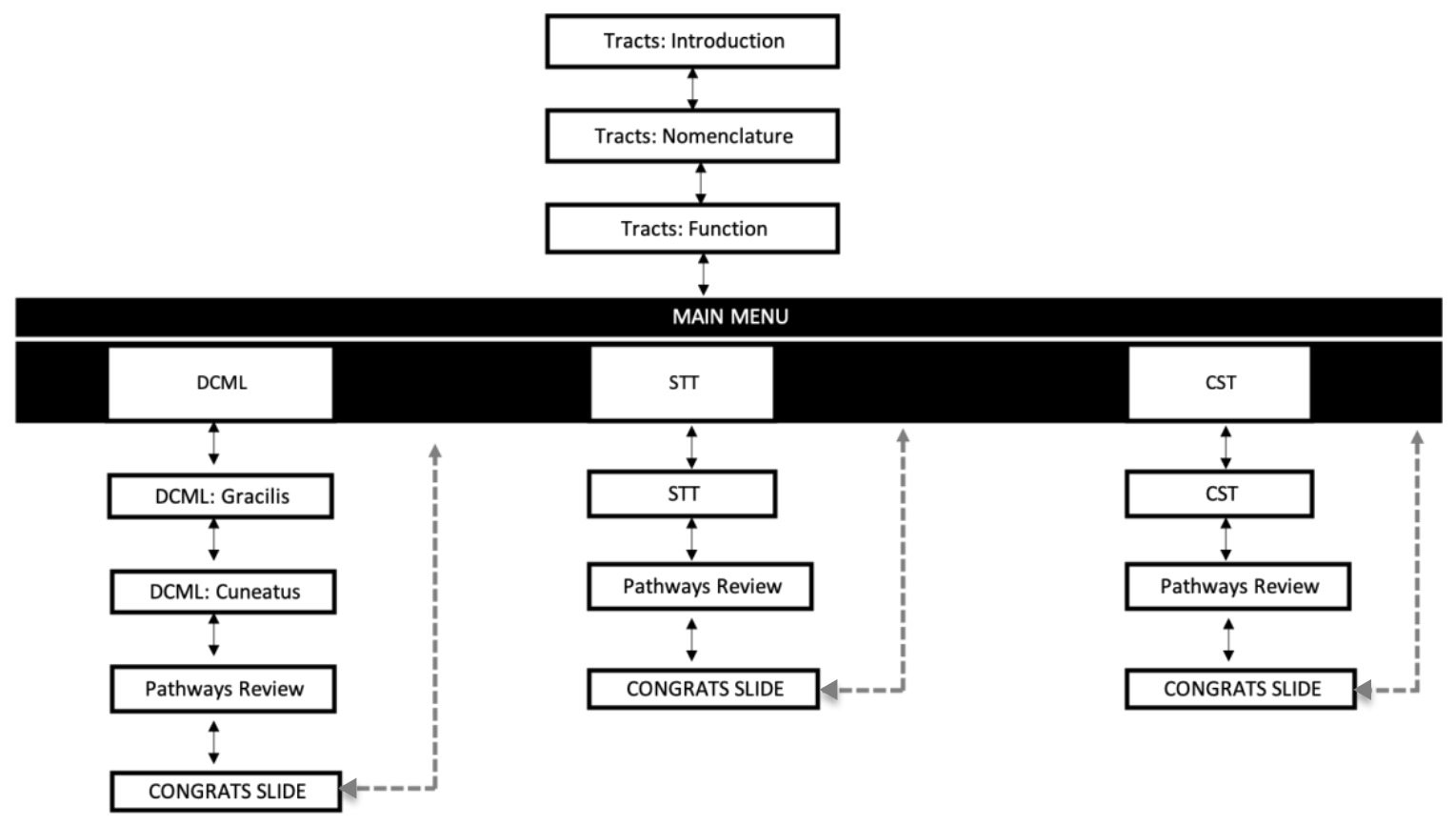


FIGURE 5.1. Non-Adaptive Module Flow Chart. Double headed arrows represent bidirectional capability of the user to progress forward and backwards as they desire. Students can access the three major tracts: dorsal column medial lemniscus (DCML), spinothalamic (STT), and corticospinal (CST) from the main menu. The congrats slide is a congratulations slide informing the students they have completed the pathway and are subsequently redirected back to the main menu. 


\section{FIGURE 5.2}

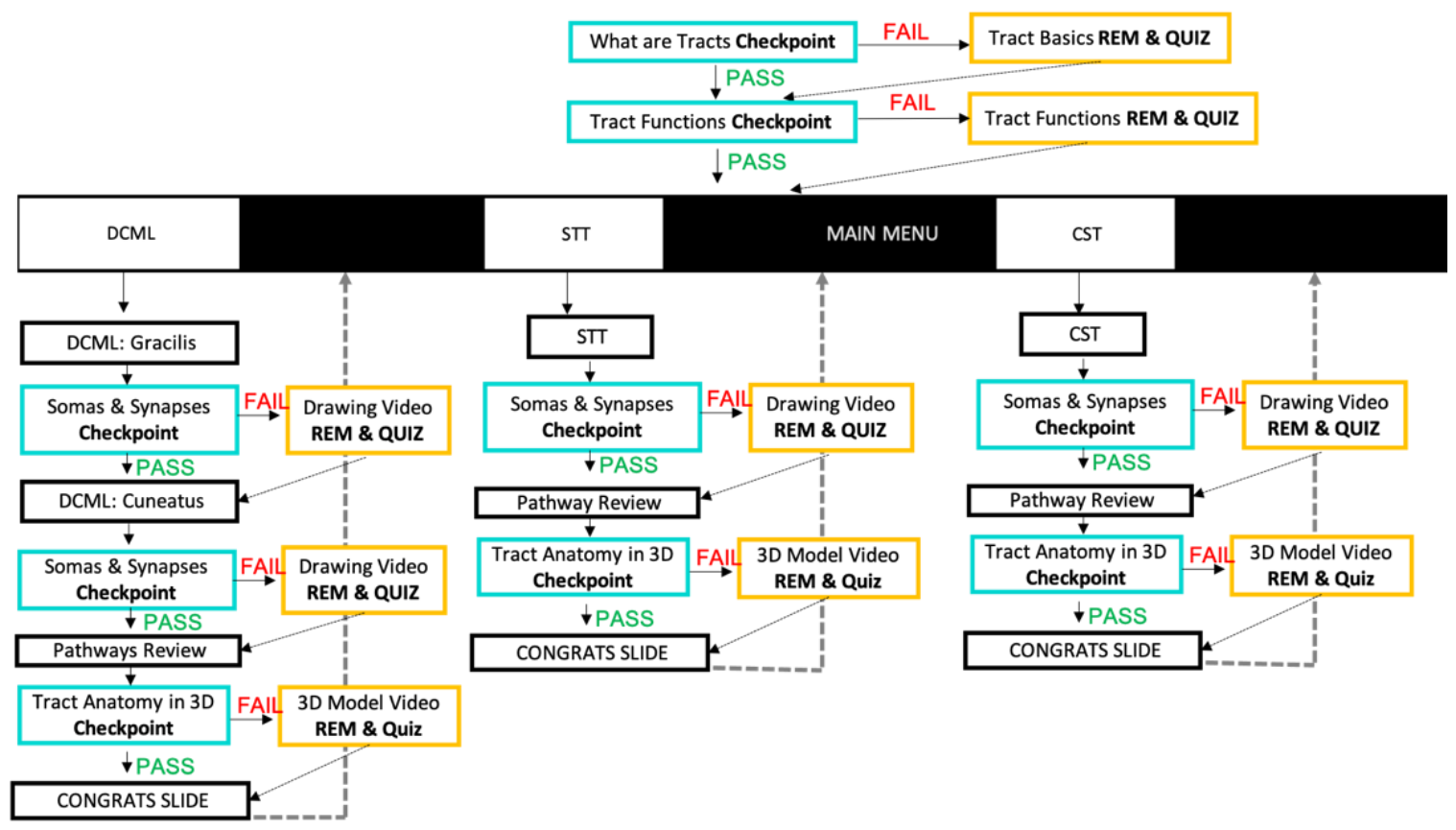


Figure 5.2. Adaptive Module Flow Chart. The blue boxes represent adaptive checkpoints and the orange boxes represent remediation loops. Checkpoints are short quizzes (3-5 questions) that require $100 \%$ competency prior to advancement to the next slide (denoted $P A S S$ ). If a student fails a checkpoint (denoted $F A I L$ ), the student is brought to remediation content (REM) and a remediation quiz (Quiz). Remediation quizzes also require $100 \%$ competency, but allow unlimited attempts and provide explanations for incorrect answers as a learning tool to guide the student. Single headed arrows represent the previous (backwards) capability have been disabled and the user is forced to progress through the module in the indicated direction. Similar to the selfdirected non-adaptive module in Figure 1, the congrats slide is a congratulations slide informing the students they have completed the pathway and are subsequently redirected back to the main menu. 


\section{FIGURE 5.3}
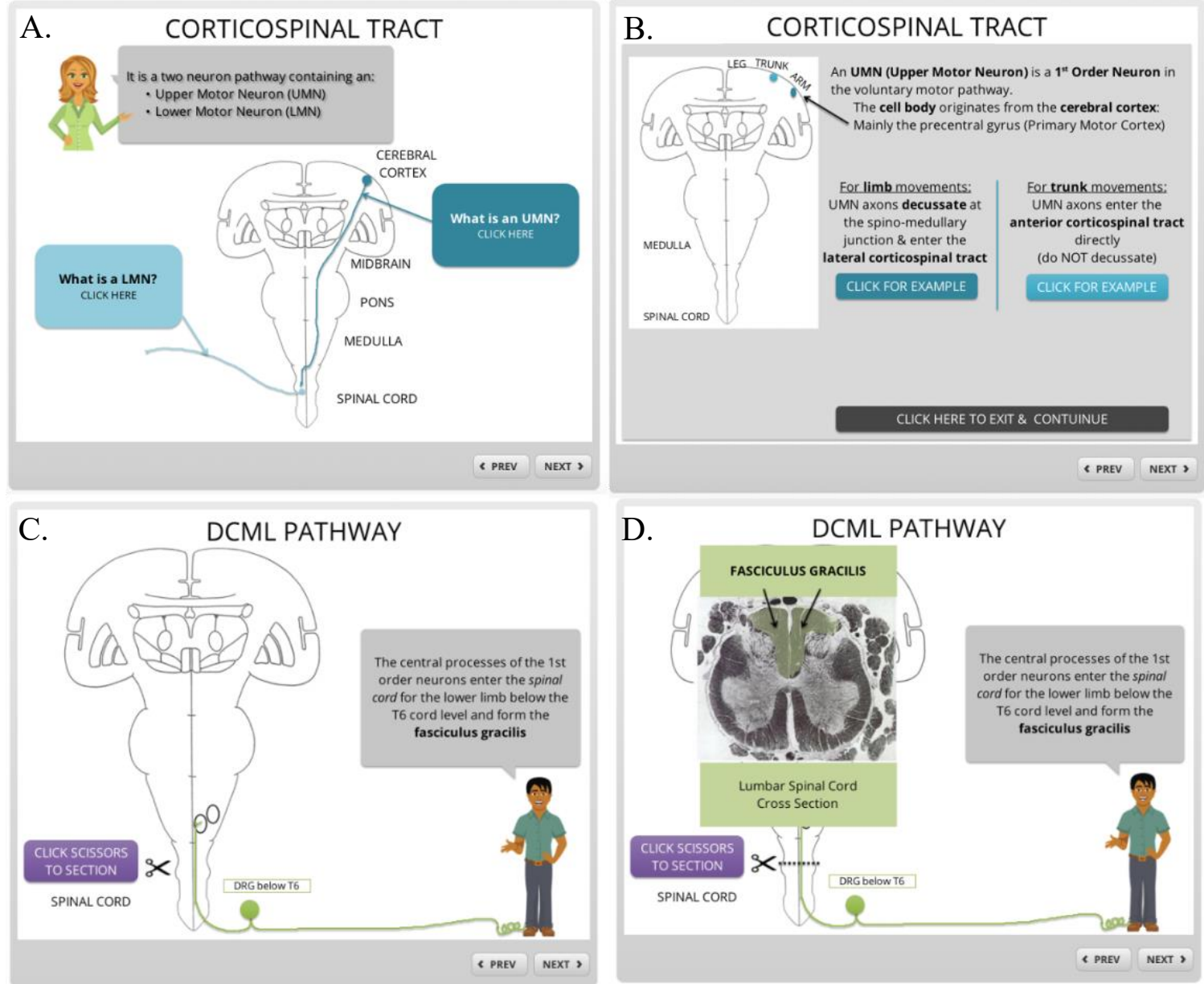
FIGURE 5.3. Content features designed to reduce neuroanxiety. Neuroanatomy vocabulary assistance and $3 D$ relationships are content features of the module designed to reduce neuroanxiety. Optional neuroanatomy vocabulary assistance is provided to students (Figure 3A and 3B) as complex jargon and lexicon were described by students as making neuroanatomy unnecessarily difficult. A student can click on the dark blue box What is an UMN? (Figure 3A) to see an optional definition (Figure 3B). 3D relationships in cross-sectional anatomy (Figure 3C and 3D) are provided along with the pathway information to provide students assistance with orienting where these three-dimensional neuroanatomical structures are located on a two-dimensional image. A student can click on the scissors (Figure 3C) to see where that pathway is located on a spinal cord crosssection (Figure 3D). 


\section{FIGURE 5.4}
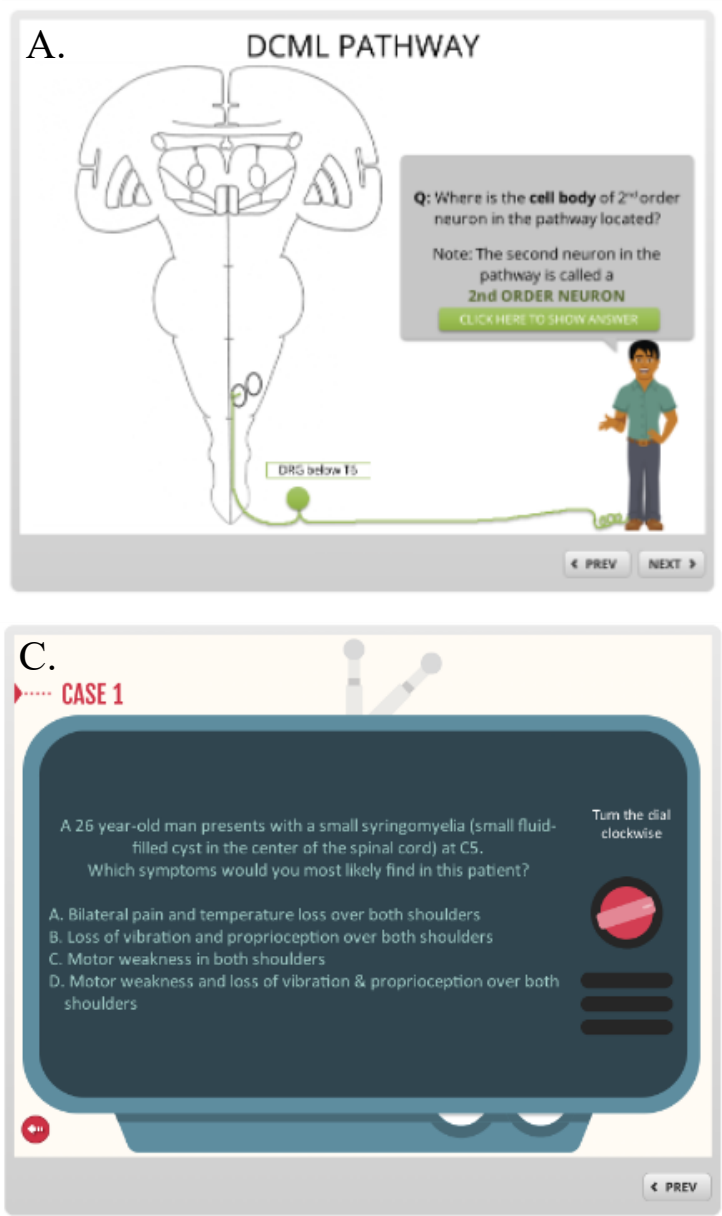

\section{E. CORTICOSPINAL TRACT}

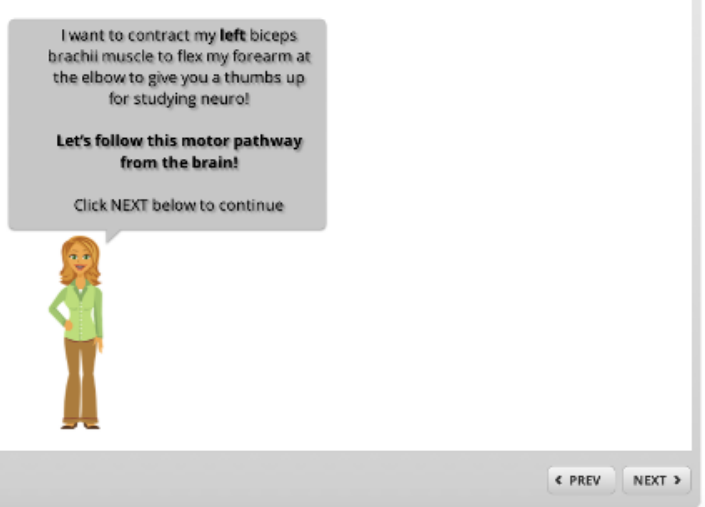

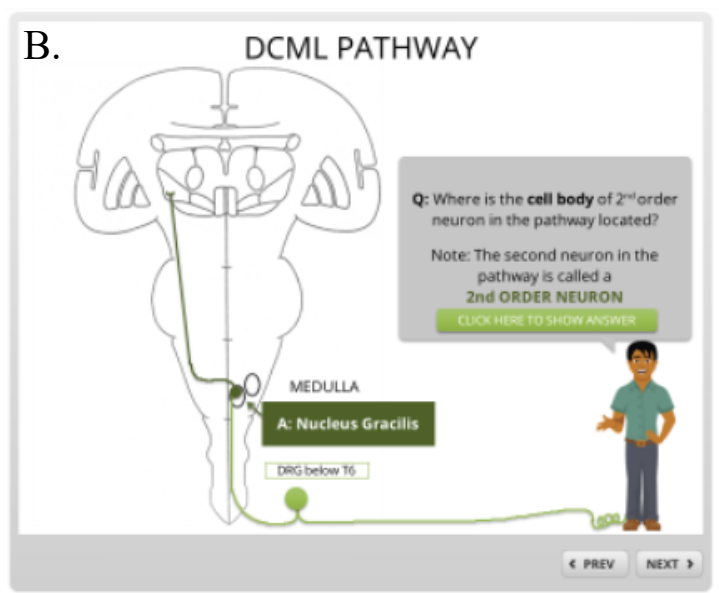

D.

1.... CASE 1
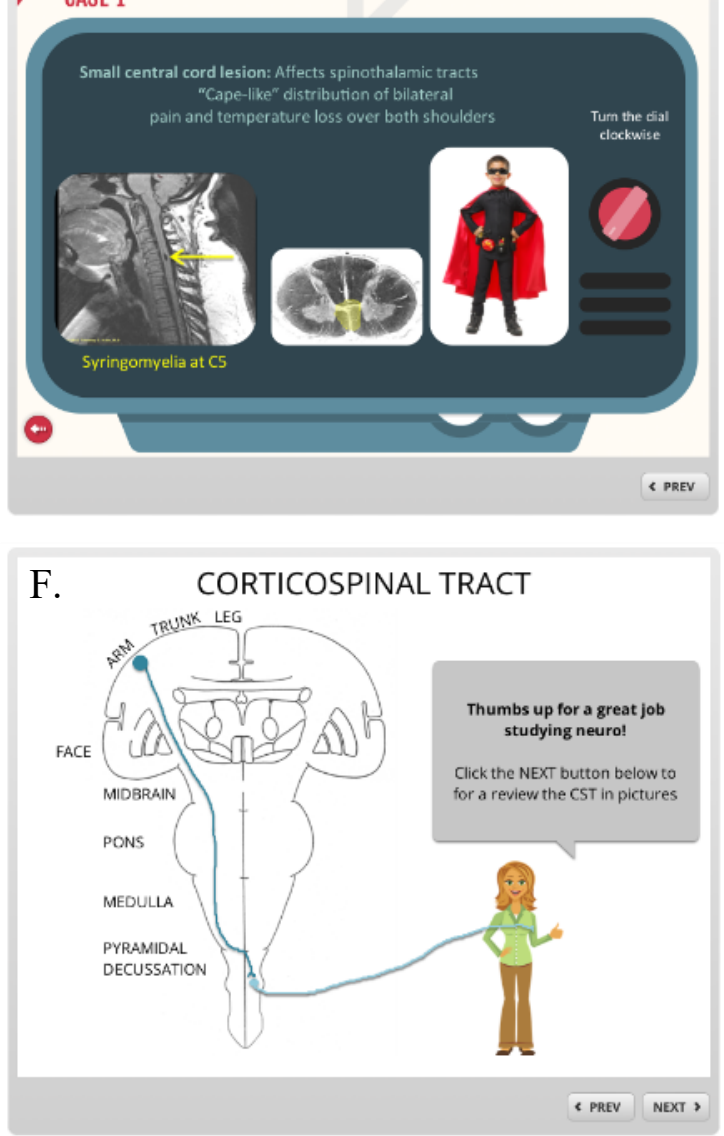
FIGURE 5.4. Instructional features designed to reduce neuroanxiety. Step-by-step instruction, clinical context, and structure-function correlation are instructional features incorporated into the module to reduce neuroanxiety. Students are shown the pathway in a step-by-step instructional manner where they answer questions along the way by clicking the Click Here to Show Answer box (Figure 4A) and being shown the answer (Figure 4B), which is the next step in the pathway. Clinical context is provided with optional spinal cord lesion cases (Figure 4C) where students work through the case until they uncover the patient's symptoms associated with that case (Figure 4D). Simple structure-function correlation is shown through an example of how the pathway may be used in everyday life. For example, a student can follow the pathway to contract the left biceps muscle (Figure 4E) and the process of outlining this pathway is completed at the end of this section (Figure 4F). 


\section{FIGURE 5.5}

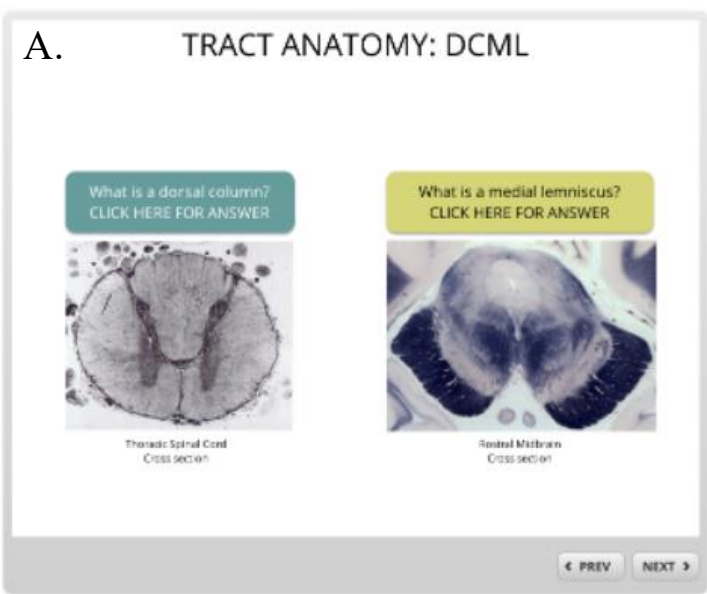

B. TRACT ANATOMY: DCML

Dorsal Column: Posterior region of the spinal cord (greent through which thes
tibers enter \& ascend
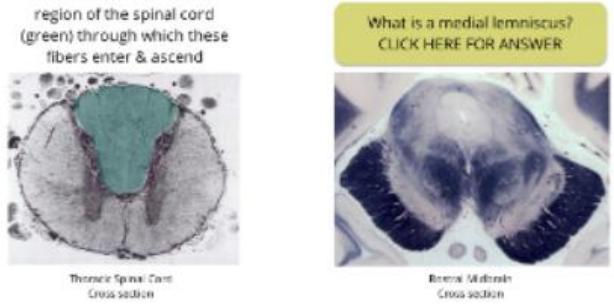

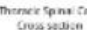

Gowasedist
C.

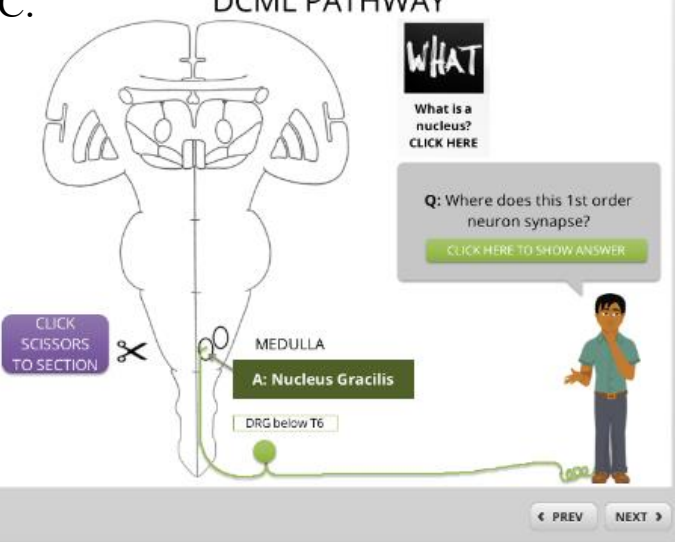

( PREV NEST 3

D.

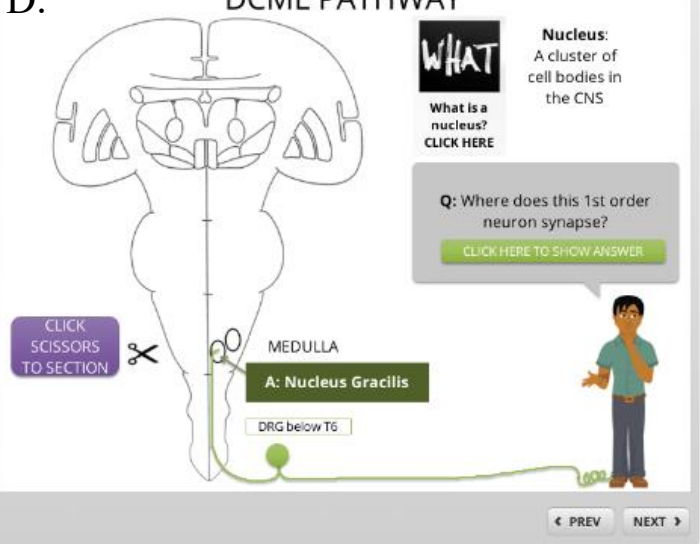


FIGURE 5.5. Communication features designed to reduce neuroanxiety. Overestimation of student knowledge was the main communication domain designed into the modules. Optional definitions of commonly used neuroanatomy phrases, for example dorsal column in the green box can be clicked on (Figure 5A) for a definition and clarification of where this is located anatomically (Figure 5B). Optional definitions are also provided within the module, for example What is a nucleus? (Figure 5C) can be clicked on and a definition is provided (Figure 5D). These optional definitions are embedded within the module to reduce the embarrassment and anxiety students may feel for not already knowing these terms. 


\section{FIGURE 5.6}

A.
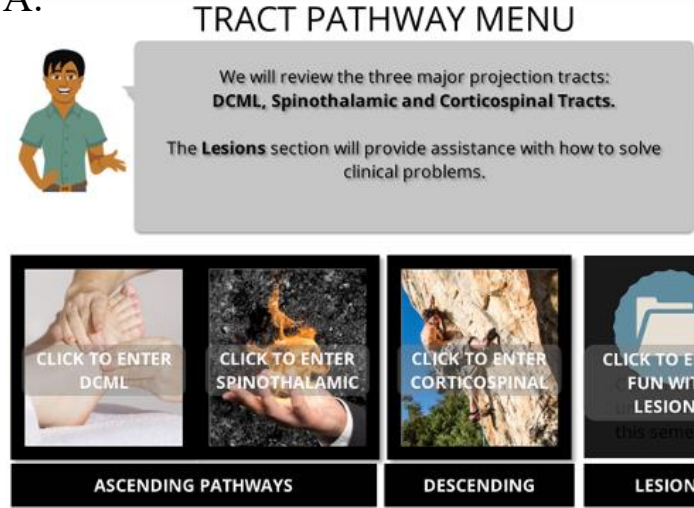

B.

\section{NEXT >}


FIGURE 5.6. Organization features designed to reduce neuroanxiety. The organization of the module was consistent to reduce neuroanxiety. The main menu (Figure 6A) contains icons to allow students to enter each pathway. Each pathway unfolds following the same order to provide organizational consistency. Additionally, students may progress at their own pace (Figure 6B); students control slide advancement by clicking on the buttons designed to reduce their anxiety while learning neuroanatomy. 


\section{FIGURE 5.7}

A.

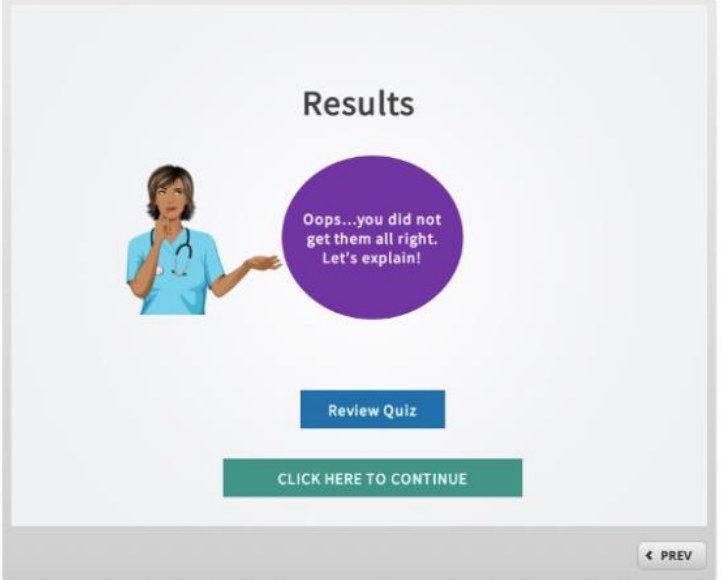

B.

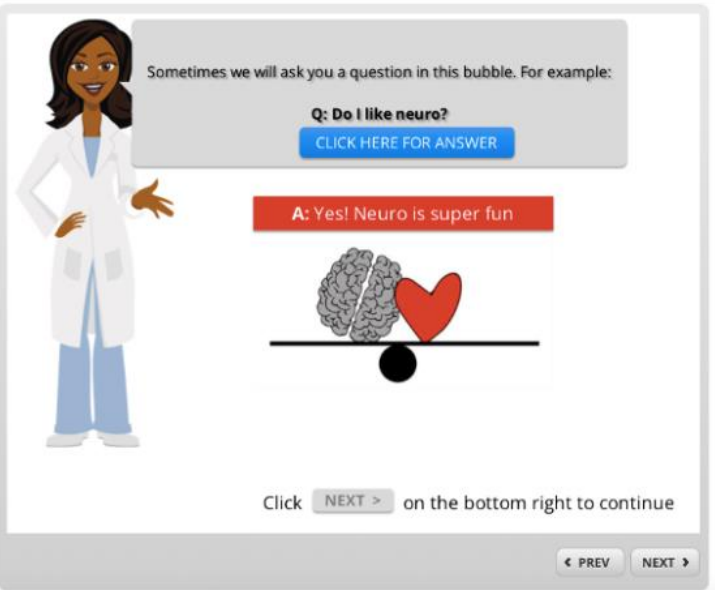


FIGURE 5.7. Emotional arousal features designed to improve neuroanatomy selfefficacy. Preventing students from becoming anxious or frustrated while using the module are features designed to keep student emotional arousal low. Soft-feedback in the form of Oops...Let's Explain along with using a neutral color (purple) instead of red is provided to students when they receive wrong answers (Figure 7A). Additionally, the module contains characters speaking directly to the students (Figure 7B). These characters act as guides assisting students during key challenging times within the module. 


\section{FIGURE 5.8}

A.

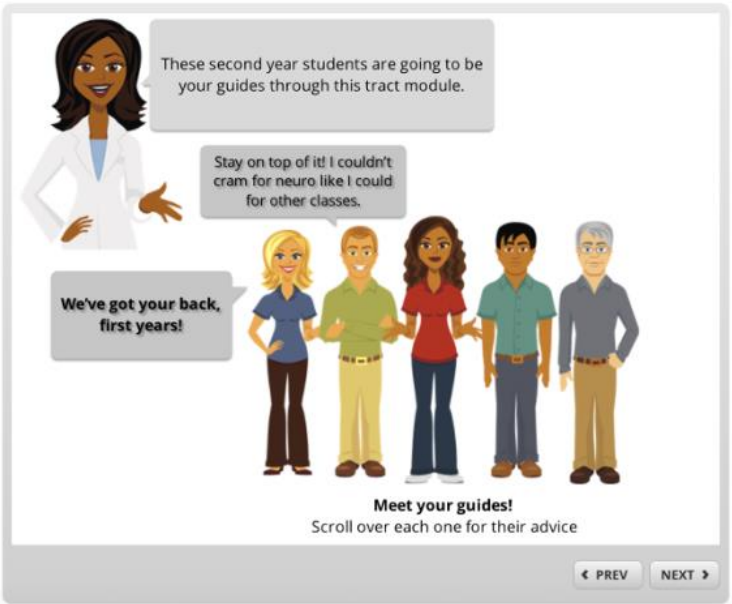

B.

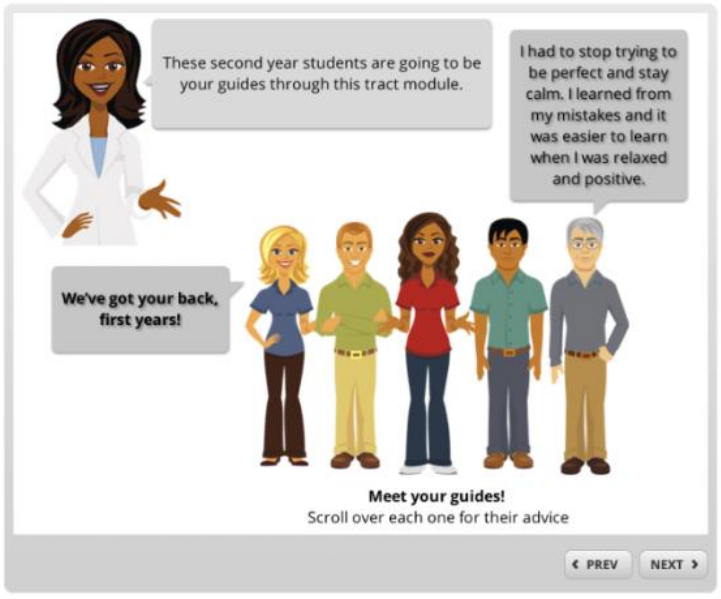


FIGURE 5.8. Verbal persuasion features designed to improve neuroanatomy selfefficacy. Verbal persuasion is provided by quotes from the characters within the module by scrolling over individual characters. For example, scrolling over the students in the green shirt (Figure 8A) and gray shirt (Figure 8B) describe hints on how best to study for neuroanatomy in the introduction. Inclusive characters representing students from different race, socioeconomic status, gender, and age were included within the module. These characters acknowledge difficult questions throughout the module and persuade the student to push on towards successful completion designed to improve student neuroanatomy self-efficacy. 


\section{FIGURE 5.9}

A.

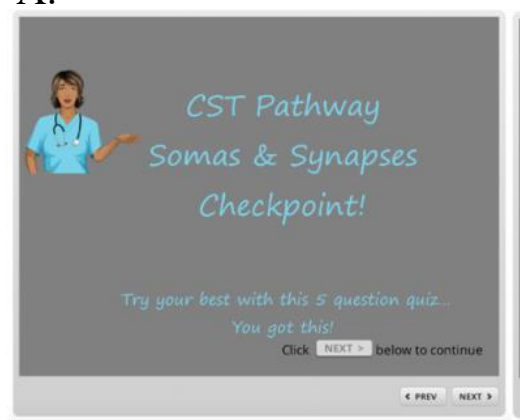

B.

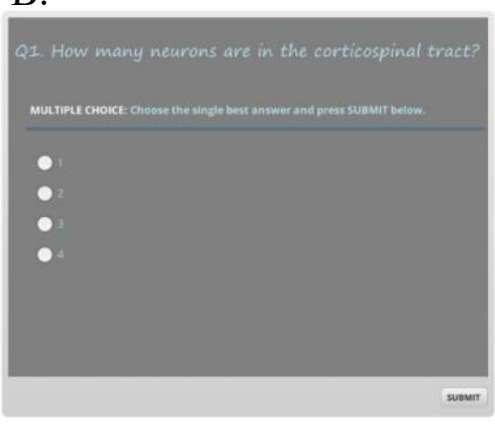

C.

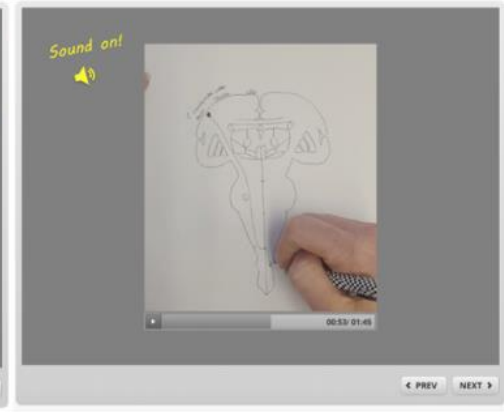


FIGURE 5.9. Somas and Synapses Checkpoint. The arrival at the Somas \& Synapses checkpoint slide (Figure 9A) after the completion of the pathway anatomy content, denotes the beginning of adaptive (competency-based) portion of the module. Competency on the Somas \& Synapses checkpoint quiz questions (Figure 9B) results in the user advancing on to the pathways review. If the user does not demonstrate $100 \%$ competency, they are brought to a Drawing Video remediation loop (Figure 9C). The Drawing Video remediation demonstrates how to draw the pathway on paper along with an audio description of the location of the cell bodies, axons, synapses, and decussation points within the tract. The student is encouraged to draw along with the video and this is intended to increase neuroanatomy self-efficacy through vicarious experience (Bandura 1986). 


\section{FIGURE 5.10}

A.

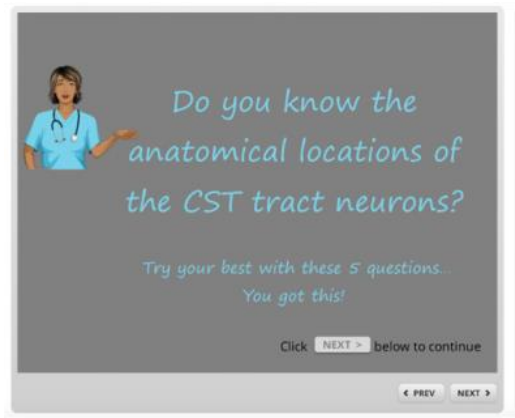

B.

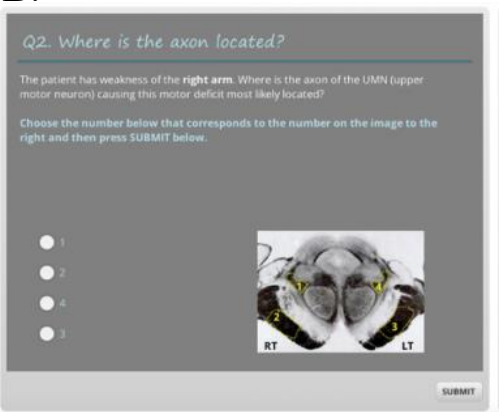

C.

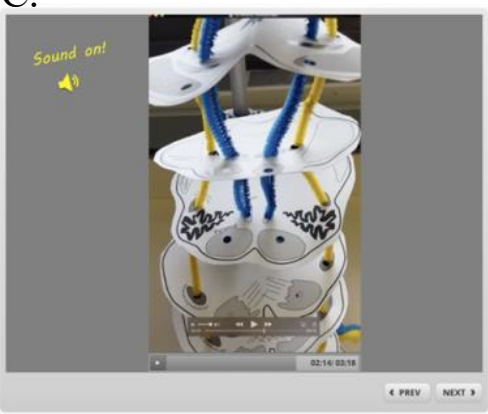


FIGURE 5.10. Tract Anatomy in 3D Checkpoint. The arrival at the Tract Anatomy in $3 D$ checkpoint slide (Figure 10A) is provided to students after the pathway review. This checkpoint assesses a student's ability to identify the location of neuronal cell bodies, axons, synapses, and decussation points on brainstem and spinal cord cross-sections (Figure 10B). If the student user does not demonstrate 100\% competency on the Tract Anatomy in $3 D$ checkpoint quiz on the first attempt, the user is brought to a $3 D$ Model Video remediation loop (Figure 10C). A 3D model was incorporated into the module from the results of Estevez et al. (2010) showing improved medical student learning outcomes using 3D models. The 3D paper model used, courtesy of Nicole Herring Ph.D., shows the cross-sectional anatomy of the spinal cord along with caudal and rostral cross sections of the medulla, pons, and midbrain. A video showing this model, along with an audio description of the neurons within the pathway, is provided to demonstrate the anatomical location of the axons and cell bodies within the pathway in a threedimensional space. 


\section{FIGURE 5.11}

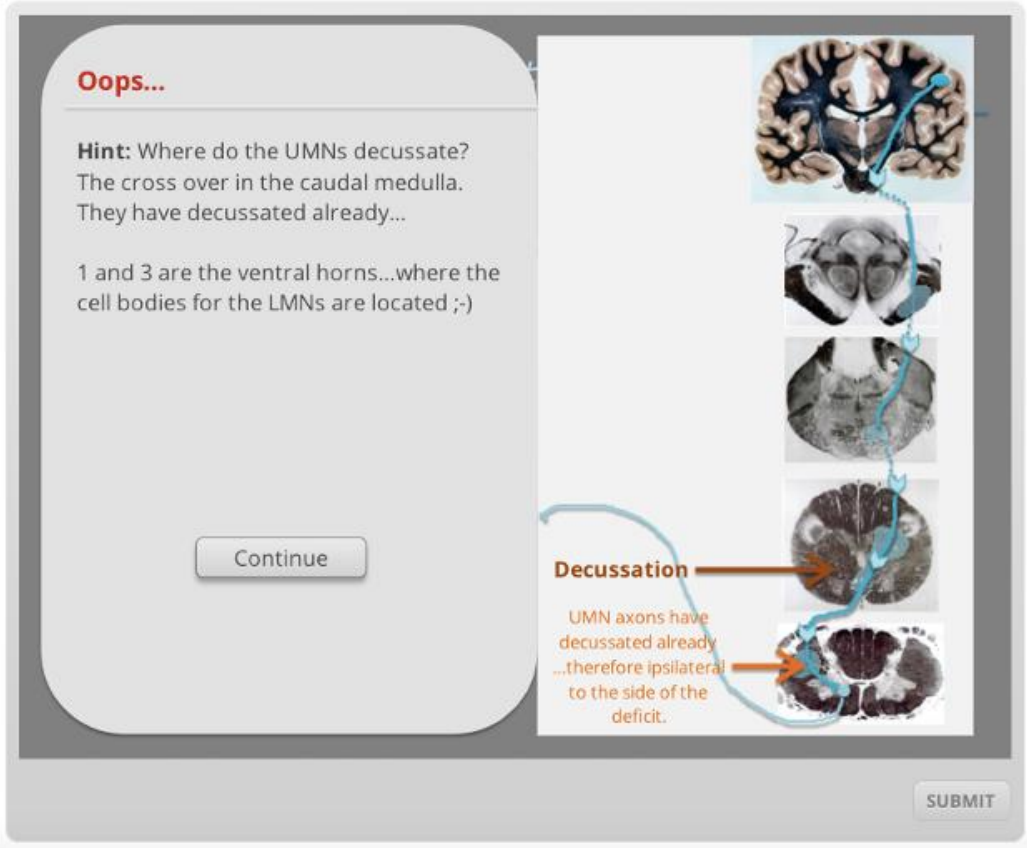




\section{FIGURE 5.11. Remediation loop quiz: Incorrect answer choice explanations.}

Explanations of incorrect answers were provided by including variables with an if/then statement revealing a hidden layer. For each specific incorrect answer choice selected, a hidden layer revels an explanation of why that answer choice was not correct and contains a hint for the student. 


\section{TABLE 5.1}

Analysis of Covariance for NSE scores by module type (treatment) with pre-test NSE scores as a covariate

\begin{tabular}{|l|c|c|c|c|c|c|}
\hline \multicolumn{1}{|c|}{ Source } & SS & df & MS & F & $\boldsymbol{p}$ & $\boldsymbol{\eta}^{\mathbf{2}}$ \\
\hline $\begin{array}{l}\text { Pre-Test NSE } \\
\text { (Covariate) }\end{array}$ & 20.73 & 1 & 20.73 & 1.48 & 0.23 & 0.019 \\
\hline $\begin{array}{l}\text { Treatment } \\
\text { (Module type) }\end{array}$ & 86.82 & 1 & 86.82 & 6.20 & 0.015 & 0.076 \\
\hline Error & 1049.98 & 75 & 14.00 & & & \\
\hline
\end{tabular}


TABLE 5.1. Analysis of Covariance (ANCOVA) for NSE scores by module type (treatment) with pre-test NSE scores as a covariate. The results of the ANCOVA showed a significant effect for module type on post-test neuroanatomy self-efficacy scores (NSES) after controlling for pre-test neuroanatomy self-efficacy scores, $F(1,75)=$ $6.2, p=0.015$. The reported partial eta squared effect size $\left(\eta^{2}=0.076\right)$ suggests a moderate practical significance. 
FIGURE 5.12

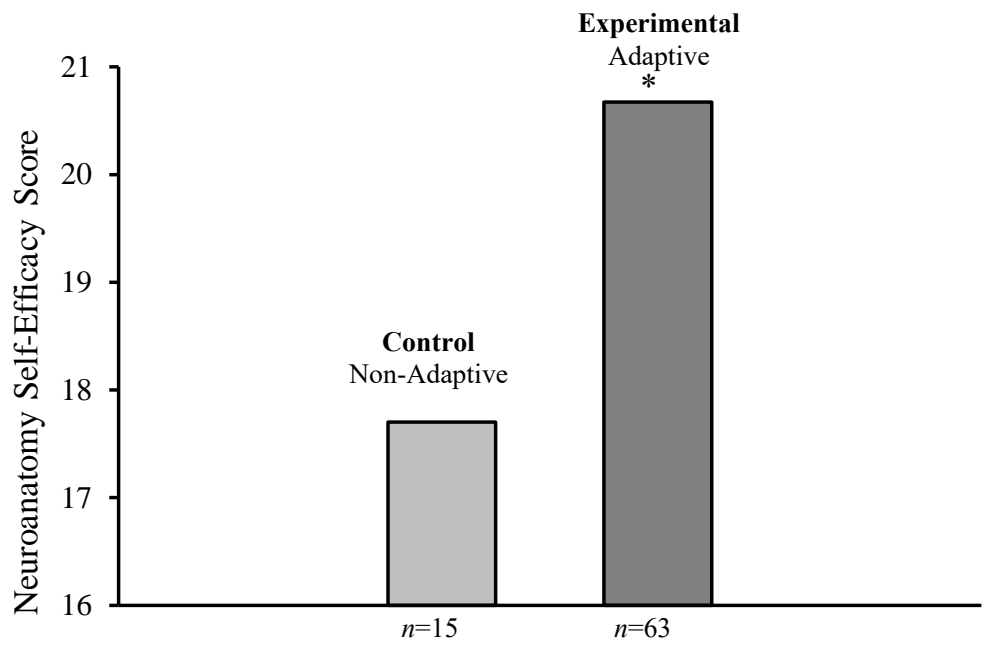

Treatment Group 
FIGURE 5.12. Effect of Module Type on NSES Scores. The asterisk (*) indicates a significant effect ( $p=0.01$ ) of module type on post-module neuroanatomy self-efficacy scores (NSES) after controlling for pre-module NSES scores, $F(1,75)=6.2, p=0.015, \eta^{2}$ $=0.076$. 
TABLE 5.2

\begin{tabular}{|l|c|c|c|c|c|c|}
\hline \multicolumn{1}{|l|}{ Tests of Between-Subjects Effects } \\
\hline \multicolumn{1}{|c|}{ Source } & SS & df & MS & F & p & $\boldsymbol{\eta}^{\mathbf{2}}$ \\
\hline Intercept & 1596.85 & 1 & 1596.85 & 53.19 & $<0.001$ & 0.542 \\
\hline $\begin{array}{l}\text { Treatment } \\
\text { (Module type) }\end{array}$ & 64.89 & 1 & 64.89 & 2.16 & 0.148 & 0.046 \\
\hline Error & 1350.96 & 45 & 30.02 & & & \\
\hline
\end{tabular}


TABLE 5.2. Tests of Between-Subjects Effects. The results of the mixed design ANOVA showed that there was no significant effect of module type $(F(1,45)=2.16, p=$ $\left.0.148, \eta^{2}=0.046\right)$ on neuroanxiety overall. Both non-adaptive and adaptive participants showed a decrease in neuroanxiety levels at the end of the first-semester of medical school. However, the adaptive module did not significantly decrease post-course neuroanxiety levels of first-year medical students compared to the non-adaptive module. 


\section{FIGURE 5.13}

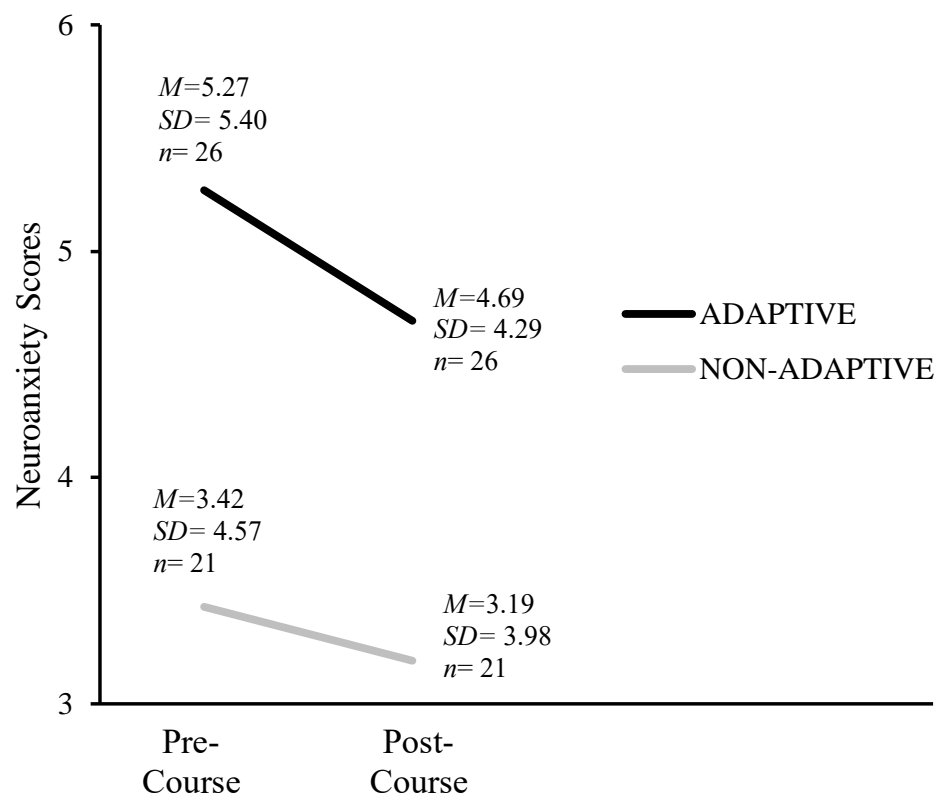


Figure 5.13. Estimated Marginal Means of Neuroanxiety Scores. The non-adaptive participants began the Fall 2018 semester with pre-course NAS scores $(M=3.42$, $S D=4.57, n=21)$; these scores were slightly lower at the end of the Fall 2018 semester $(M=3.19, S D=3.98, n=21)$. While the adaptive group appears to have slightly larger decreases in neuroanxiety pre- and post-course than the non-adaptive group upon visual inspection, these differences were not significant $\left(F(1,45)=.052, p=0.82, \eta^{2}=0.001\right)$. The adaptive module did not significantly decrease post-course neuroanxiety levels of first-year medical students compared to the non-adaptive module. 


\section{CHAPTER 6}

\section{DISCUSSION}

Neuroanxiety, also termed neurophobia, and a potentially related construct, neuroanatomy self-efficacy, in medical students learning neuroanatomy within an integrated curriculum were explored in the studies in this dissertation. Scales were developed to measure both neuroanxiety and neuroanatomy self-efficacy in order to assess the effects of a learning intervention designed to reduce neuroanxiety and improve neuroanatomy self-efficacy. An adaptive computer-based eLearning intervention, that could be efficiently implemented within the time constraints of an integrated curriculum, was developed. The effect of the adaptive eLearning intervention on both neuroanxiety and neuroanatomy self-efficacy was compared to a non-adaptive eLearning intervention. Both modules were developed to help ease neuroanxiety and bolster neuroanatomy selfefficacy; however, only the adaptive module significantly increased neuroanatomy selfefficacy. Both adaptive and non-adaptive modules slightly decreased neuroanxiety, although none of those data proved significant. These findings suggest neuroanatomy self-efficacy may be influenced, more than neuroanxiety, by an adaptive eLearning intervention.

The findings in Chapter 2 reveal the presence of neurophobia at our institution through an open dialogue with first-year medical students. The qualitative data from the 
discussion group suggest ways in which neuroanatomy instruction can be improved in the four domains of content, instruction, communication, and organization. Neuroanatomy content could be improved through a progression of clinical case complexity throughout the semester, more frequent distribution of concise neuroanatomy terms lists, and neuroanatomy laboratory videos to explain anatomical variability. Instruction may be improved through greater structure-function correlation and guiding students through the step-by-step thought process of solving clinical case problems. Communication between students and faculty needs improvement by making it clear what is expected of students, increased faculty to faculty communication in a team-taught course, and better consistency in neuroanatomy lab instruction. Improved organization of neuroanatomy content with more applicable timing of the integrated anatomy content and more even distribution of neuroanatomy material throughout the semester. This study contributes depth to the literature by describing specific instructional strategies to improve neuroanatomy teaching, in the four domains described above, that can be used to minimize the inadequate teaching described in previous studies (Flanagan et al, 2007; Youssef, 2009; Zinchuk et al., 2010; Abulaban et al., 2015). This study was limited by the large size and time constraints of scheduling one discussion group section. Further qualitative study, with smaller, successive focus groups more diligently following grounded theory methodology until there is redundancy in student responses, will allow for more comprehensive qualitative findings. Additionally, a future qualitative study with moderators outside the instructional team would further minimize moderator bias as students may have felt restrained from criticizing, or perhaps even, felt compelled to praise the instruction. 
As discussed in Chapter 3, we developed a 7-item scale to measure neuroanxiety, explored the level of neuroanxiety of our medical students, and the predictors of neuroanxiety at our institution. Our findings suggest the Neuroanxiety Scale (NAS) is a short, yet informative, scale to measure one construct, neuroanxiety, based on the framework of the GAD-7 (Spitzer et al., 2006). It shows high internal consistency (Cronbach's $\alpha=0.91$ ), convergent validity with correlated scores on the Neuroanatomy Emotions and Beliefs Scales, and discriminant validity through negative correlation with the Neuroanatomy Self-Efficacy Scale (NSES). Our findings suggest both upper-class and female medical students exhibited greater neuroanxiety than first-year and male medical students. Female medical students, even with premedical neuroscience and/or neuroanatomy experience, are trending towards greater levels of neuroanxiety than their male peers. Additionally, this study found female gender, lecture difficulties, lack of clinical case integration and 3D models to learn neuroanatomy predict neuroanxiety at our institution. This study contributes a novel scale to measure neuroanxiety in medical students and to the depth of the literature on contributors of neurophobia. The novel finding that premedical neuroanatomy and/or neuroscience experience predicts lower neuroanxiety in medical school adds depth to the existing neurophobia literature. This study was limited by our sample size. Our data on neuroanxiety and gender show female medical students are trending to self-report greater neuroanxiety levels, even with premedical experiences, although not significant $(p=0.06)$. Another limitation of our study was the inability to screen our students for anxiety disorders, due to lack of mental health professionals on the research team, to control for medical students with an anxiety disorder when exploring neuroanxiety levels. 
The focus of Chapter 4 was to investigate which, if any, premedical neuroanatomy and/or neuroscience experiences are associated with increased neuroanatomy self-efficacy. First, scale development of the 13-item neuroanatomy selfefficacy scale (NSES) was modified from an anatomy self-efficacy scale (Burgoon et al., 2012) and was hypothesized to have three dimensions - perceived skill (magnitude), transferability (generality), and perseverance (strength) — based on self-efficacy theory (Pajares, 1996; Bandura, 2006; Bandura, 2012; Schunk \& DiBenedetto, 2016). Our findings suggest the NSES is a useful 13-item scale to measure the three-dimensional construct neuroanatomy self-efficacy. Exploratory factor analysis showed a three-factor solution with transferability, perseverance, and perceived skill; each subscale showed high internal reliability (Cronbach's $\alpha=0.925,0.894$, and 0.81 respectively). Second, two distinct different types of premedical neuroscience and/or neuroanatomy experiences were found to have an effect on neuroanatomy self-efficacy: (1) combined premedical molecular neuroscience and neuroanatomy course enrollment, and (2) indirect neuroanatomy exposure as part of a premedical gross anatomy course. An interesting secondary finding of this study was premedical exposure to neuroscience and/or neuroanatomy appears to increase neuroanatomy self-efficacy of female medical students while their male colleagues do not show the same pattern of improved neuroanatomy self-efficacy with premedical experiences. This study contributes a novel scale to measure neuroanatomy self-efficacy in medical students to the literature. Additionally, the novel findings that indirect neuroanatomy exposure as part of a premedical gross anatomy course and female medical students seem to benefit more from premedical experiences adds breadth to the academic self-efficacy literature. Considering the gender 
disparity in neurology, and associated decline in neurologists, these findings support youth STEM neuroscience initiatives to give female students increased pre-medical experiences with neuroscience and neuroanatomy to attract them to the field. One of the limitations of our study was the exploratory factor analysis suggest this survey may benefit from further development. Items 5 and 9 were troublesome in that they both demonstrated cross-loadings, or groupings with more than one dimension. This scale could benefit from another round of data collection, with rewording of scale items, and factor analysis to clarify the factor structure.

The exploration of comparing adaptive and non-adaptive eLearning on neuroanxiety and neuroanatomy self-efficacy in Chapter 5 showed adaptive eLearning only significantly influenced neuroanatomy self-efficacy. The adaptive eLearning intervention slightly reduced medical student neuroanxiety, but these results were not significant. Academic self-efficacy is suggested to moderate, or reduce, academic anxiety in two previous studies (Nie et al., 2011; Barrows et al., 2013). The Nie et al. (2011) study focused on the moderating effect of academic self-efficacy on academic anxiety in a high-stakes environment, such as neuroanatomy in medical school. Our findings add depth to the existing literature on academic self-efficacy and anxiety by suggesting that an adaptive eLearning intervention may improve neuroanatomy self-efficacy in a highstakes environment, such as medical school. Limitations of this study were the low survey response rates, and differential administration, to both the pre-course and postcourse neuroanxiety scale (NAS) and the pre-module and post-module neuroanatomy self-efficacy scale (NSES). This study may benefit from another round of data collection with a larger sample size that may explain differences that did not appear in the small 
sample population. The timing of the NAS survey administration differed from the NSES scale; the NAS was administered at the beginning and end of the semester while the NSES was administered before and after completion of the module. The differential timing of survey administration, intended to reduce survey fatigue, may have unintentionally biased the results. It may be that the close timing between the completion of the eLearning module and the completion of the NSES survey strengthened the relationship between these factors. Completing the NSES immediately after finishing the module may reflect a more accurate representation of a student's neuroanatomy selfefficacy measurement as a result of the module. In contrast, the longer length of time passing between the completion of the eLearning module and the completion of the NAS survey (approximately 9 weeks) may have contributed to the lack of statistical significance in this relationship. Maturation of students learning neuroanatomy content throughout the semester may have resulted in lower neuroanxiety post-course scores as a function of learning neuroanatomy with the passage of time. Additionally, this differential timing of survey administration may have resulted in mismeasurement of neuroanxiety. Since nine weeks had passed, it is uncertain if the feelings students reported at the end of the semester would have been the same as those they may have felt at the time of the module. Overall, adaptive eLearning for neuroanatomy was well received by our students and showed improved neuroanatomy self-efficacy.

Collectively, the data from this dissertation suggest adaptive eLearning may preferentially improve neuroanatomy self-efficacy over reducing neuroanxiety. Our data suggest, together with the current literature of the potential moderating effect of academic self-efficacy on academic anxiety (Nie et al., 2011; Barrows et al., 2013), perhaps future 
educational interventions to address neuroanxiety (neurophobia) might focus on improving neuroanatomy self-efficacy to indirectly moderate neuroanxiety. This work supports an exploration of this hypothesis in a future study. This work also supports further development and implementation of neuroanatomy adaptive eLearning interventions for use within the time constraints of an integrated curriculum. Additionally, our data show premedical neuroanatomy experiences, particularly associated with a cadaveric gross anatomy laboratory, may increase neuroanatomy selfefficacy. This finding, taken together with our novel finding that premedical neuroanatomy and/or neuroscience experience also predicts lower neuroanxiety in medical school, suggests neuroanatomy self-efficacy may be an even more important construct to explore with the aim of mitigating the effects of neuroanxiety. Lastly, our findings suggest that premedical neuroscience and/or neuroanatomy experience appears to preferentially effect female students, however, more study is needed in this area. This work may support youth STEM neuroscience initiatives to give girls increased premedical experiences with neuroscience and neuroanatomy to attract more women to neurology - considering the gender disparity in neurology and concurrent shortage of neurologists. 


\section{REFERENCES}

AAN. (2013). The doctor won't see you now? Study: US facing a neurologist shortage. ScienceDaily. Retrieved from:

www.sciencedaily.com/releases/2013/04/130417164444.htm [accessed 24 June 2020]

AAN. (2017). Gender disparity task force report. American Academy of Neurology. Retrieved from: https://www.aan.com/conferences-community/memberengagement/Learn-About-AAN-Committees/committee-and-task-forcedocuments/gender-disparity-task-force-report/ [accessed 21 September 2019]

Abdi, H., \& Williams, L.J. (2010). Principal component analysis. WIREs Comp Stat, 2: 433-459. https://doi.org/10.1002/wics.101

Abulaban, A.A., Obeid, T.H., Algahtani, H.A., Kojan, S.M., \& Al-Khathaami, A.M. (2015). Neurophobia among medical students. Neurosciences, 20, 37-40.

Abushouk, A.I. \& Duc, N.M. (2016). Curing neurophobia in medical schools: evidencebased strategies. Medical Education Online, 21 (32476).

http://dx.doi.org/10.3402/meo.v21.32476.

Akle V, Peña-Silva RA, Valencia DM, Rincón-Perez CW. (2018). Validation of clay modeling as a learning tool for the periventricular structures of the human brain. Anat Sci Educ, 11(2), 137-145. doi:10.1002/ase.1719

Alhadabi, A., \& Karpinski, A.C. (2020). Development And Psychometric Assessment Of The Social Media Motives Scale Among University Students. European Journal of Educational Research, 9(2), 835-851. https://doi.org/10.12973/eu-jer.9.2.835

Allen LK, Eagleson R, de Ribaupierre S. (2016). Evaluation of an online threedimensional interactive resource for undergraduate neuroanatomy education. Anat Sci Educ, 9(5), 431-439. doi:10.1002/ase.1604

Altman, D.G. (1991). Practical statistics for medical research. Chapman and Hall, London. https://doi.org/10.1002/sim.4780101015

Alzerwi NAN. (2020). Grit Scores: A Predictor of Medical School Success? [Response to 
Letter]. Adv Med Educ Pract, 11, 537-538

https://doi.org/10.2147/AMEP.S274616

AMA. (2014). 2008 - 2014 AAMC/AMA National GME Censuses. Retrieved from: https://members.aamc.org/eweb/upload/Physician\%20Specialty\%20Databook\%2 02014.pdf [accessed 26 June 2020]

American Educational Research Association, American Psychological Association, National Council on Measurement in Education, Joint Committee on Standards for Educational and Psychological Testing (U.S.). (2014). Standards for educational and psychological testing. Washington, DC: AERA.

American Psychiatric Association. (2013). Diagnostic and statistical manual of mental disorders ( $5^{\text {th }}$ ed.). https://doi.org/10.1176/appi.books. 9780890425596

Anwar, K., Shaikh, A.K., Sajid, M.R., Cahusac, P., Norah A. \& Shedoukhy, A. (2015). Tackling student neurophobia in neurosciences block with team-based learning. Medical Education Online, 20, 1, DOI: 10.3402/meo.v20.28461

Arantes, M., Andrade, J. P., Barbosa, J., \& Ferreira, M. A. (2020) Curricular changes: the impact on medical students knowledge of neuroanatomy. BMC medical education, 20(1), 20. https://doi.org/10.1186/s12909-019-1907-1

Artino, A. (2012). Academic self-efficacy: From educational theory to instructional practice. Perspectives on medical education, 1, 76-85. 10.1007/s40037-012-0012-5.

Association of American Medical Colleges. 2020. Weeks of Instruction and Contact Hours Required at US Medical Schools. AAMC Curriculum Inventory, 20182019. [https://www.aamc.org/data-reports/curriculum-reports/interactivedata/weeks-instruction-and-contact-hours-required-us-medical-schools]. Accessed [19 June 2020].

Bandura, A. (1977). Self-efficacy: Toward a unifying theory of behavioral change. Psychol Rev, 84, 191-215.

Bandura, A. (1986). Social foundations of thought and action-A social cognitive theory. Englewood Cliffs, NJ: Prentice-Hall.

Bandura, A., Barbaranelli, C., Caprara, G.V., \& Pastorelli, C. (1996). Multifaceted Impact of Self-Efficacy Beliefs on Academic Functioning. Child Development, 67, 1206-1222. https://doi.org/10.1111/j.1467-8624.1996.tb01791.x

Bandura, A. (2001). Social Cognitive Theory: An Agentic Perspecive. Annual Review of Psychology, 52, 1-26. https://doi.org/10.1146/annurev.psych.52.1.1 
Bandura, A. (2005). The Evolution of Social Cognitive Theory. In K. G. Smith, \& M. A. Hitt (Eds.), Great Minds in Management (pp. 9-35). Oxford: Oxford University Press.

Bandura, A. (2006). Guide for constructing self-efficacy scales. In F. Pajares, \& T. Urdan (Eds.), Adolescence and education: Vol. 5. Self efficacy and adolescence (pp. 307-337). Greenwich, CT: Information Age.

Bandura, A. (2012). On the Functional Properties of Perceived Self-Efficacy Revisited. Journal of Management, 38(1), 9-44. https://doi.org/10.1177/0149206311410606

Barrett, L. F. (2004). Feelings or Words? Understanding the Content in Self-Report Ratings of Experienced Emotion. Journal of Personality and Social Psychology, 87(2), 266-281. https://doi.org/10.1037/0022-3514.87.2.266

Barrows, J., Dunn, S., \& Lloyd, C.A. (2013). Anxiety, Self-Efficacy, and College Exam Grades. Universal Journal of Educational Research 1(3), 204-208. DOI: 10.13189/ujer.2013.010310

Bartlett, M.S. (1950). Test of significance in factor analysis. British Journal of Statistical Psychology, 3, 77-85. https://doi.org/10.1111/j.2044-8317.1950.tb00285.x

Bauer GR, Braimoh J, Scheim AI, \& Dharma C. (2017) Transgender-inclusive measures of sex/gender for population surveys: mixed-methods evaluation and recommendations. PLoS ONE 12(5): e0178043. https://doi.org/10.1371/journal.pone.0178043

Beck, A. T., Epstein, N., Brown, G., \& Steer, R. A. (1988). An inventory for measuring clinical anxiety: psychometric properties. Journal of consulting and clinical psychology, 56(6), 893-897. https://doi.org/10.1037//0022-006x.56.6.893

Berwick, Donald M. MD, MPP; Finkelstein, Jonathan A. MD, MPH Preparing Medical Students for the Continual Improvement of Health and Health Care: Abraham Flexner and the New "Public Interest", Academic Medicine: September 2010 Volume 85 - Issue 9 - p S56-S65 doi: 10.1097/ACM.0b013e3181 ead779.

Bierer, S.B., Prayson, R.A., \& Dannefer, E.F. (2015). Association of research selfefficacy with medical student career interests, specialization, and scholarship: a case study. Adv in Health Sci Educ, 20, 339-354. DOI 10.1007/s10459-014-95317

Blanch-Hartigan, D. (2011). Medical students' self-assessment of performance: Results from three meta-analyses. Patient education and counseling, 84, 3-9. 10.1016/j.pec.2010.06.037.

Brauer, D.G. \& Ferguson, K.J. (2015). The integrated curriculum in medical education: 
AMEE Guide No. 96, Medical Teacher, 37(4), 312-322.

http://dx.doi.org/10.3109/0142159X.2014.970998

Brown, G. T. L., Andrade, H. L., \& Chen, F. (2015) Accuracy in student self-assessment: directions and cautions for research. Assessment in Education: Principles, Policy \& Practice, 22(4), 444-457. DOI: 10.1080/0969594X.2014.996523

Buja L.M. (2019). Medical education today: all that glitters is not gold. BMC Med Educ, 19(110), doi: 10.1186/s12909-019-1535-9.

Burgoon, J.M., Meece, J.L. and Granger, N.A. (2012), Self-efficacy's influence on student academic achievement in the medical anatomy curriculum. Anat Sci Ed, 5, 249-255. https://doi.org/10.1002/ase. 1283

Burns, E.C., Martin A.J., Kennett R.K., Pearson J., Munro-Smith V. (2021). Optimizing science self-efficacy: A multilevel examination of the moderating effects of anxiety on the relationship between self-efficacy and achievement in science. Contemporary Educational Psychology, 64 (101937), https://doi.org/10.1016/j.cedpsych.2020.101937

Carpenter, S. (2018). Ten steps in scale development and reporting: a guide for researchers. Communication Methods and Measures, 12(1), 25-44. htpps://doi.org/10.1080/19312458.2017.1396583

Chen H., van den Broek C., Sjoukje W.E., ten Cate O. (2015). The Case for Use of Entrustable Professional Activities in Undergraduate Medical Education, Academic Medicine, 90(4), 431-436 DOI: 10.1097/ACM.0000000000000586.

Choi-Lundberg D.L., Al-Aubaidy, H.A., Burgess, J. R., Clifford, C. A., Cuellar, W. A., Errey, J. A., Harper, A. J., Malley, R. C., Ross, R. M., Williams, A. M. \& Hays, R. (2020). Minimal effects of reduced teaching hours on undergraduate medical student learning outcomes and course evaluations, Medical Teacher 42(1), 58-65. DOI: $10.1080 / 0142159$ X.2019.1652258

Clark, L. A., \& Watson, D. (1995). Constructing validity: Basic issues in objective scale development. Psychological Assessment, 7, 309-319. DOI: $10.1037 / 10403590.7 .3 .309$

Cohen, J. (1992). Statistical Power Analysis. Current Directions in Psychological Science, 1(3), 98-101. https://doi.org/10.1111/1467-8721.ep10768783

Combes, J.R., \& Arespacochaga, E. (2012) Physician Competencies for a 21st Century Health Care System. Journal of Graduate Medical Education, 4 (3): 401-405.

Cooke, M., Irby, D. M., Sullivan, W., \& Ludmerer, K. M. (2006). American medical 
education 100 years after the Flexner report. The New England journal of medicine, 355(13), 1339-1344. https://doi.org/10.1056/NEJMra055445

Cronbach, L.J. (1951). Coefficient alpha and the internal structure of tests. Psychometrika (16): 297-334. https://doi.org/10.1007/BF02310555

Crowley, WF and Gusella, JF. (2009). Changing Models of Biomedical Research. Science Translational Medicine, 1(1). DOI: 10.1126/scitranslmed.3000124.

Cuddy, M. M., Swanson, D. B., Drake, R. L., Pawlina, W. (2013). Changes in anatomy instruction and USMLE performance: empirical evidence on the absence of a relationship. Anat Sci Educ 6, 3-10.

Daoud, J. (2017). Multicollinearity and regression analysis. J. Phys.: Conf. Ser. 949(012009), 1-6. DOI:10.1088/1742-6596/949/1/012009.

Denzin, N.K. (2012). Triangulation 2.0. J Mix Methods Res 6(2), 80-88.

Dixon, A., Silva, N.A., Sotayo, A., \& Mazzola, C.A. (2019). Female Medical Student Retention in Neurosurgery: A Multifaceted Approach. World Neurosurgery, 122, 245-251. https://doi.org/10.1016/j.wneu.2018.10.166.

Drake, R.L., Lowrie, D., Jr \& Prewitt, C.M. (2002). Survey of gross anatomy, microscopic anatomy, neuroscience, and embryology courses in medical school curricula in the United States. Anat. Rec., 269, 118122. https://doi.org/10.1002/ar.10079

Drake, R. L., McBride, J. M., Lachman, N., \& Pawlina, W. (2009). Medical education in the anatomical sciences: the winds of change continue to blow. Anatomical sciences education, 2(6), 253-259. https://doi.org/10.1002/ase.117

Duckworth, A. L., Peterson, C., Matthews, M. D., \& Kelly, D. R. (2007). Grit: Perseverance and passion for long-term goals. Journal of Personality and Social Psychology, 92(6), 1087-1101. https:// https://doi.org/10.1037/00223514.92.6.1087

Duque-Parra, J.E. (2001). Functional neuroanatomy: the first daughter of neuroscience and the mother of neural science. Anat. Rec., 265: 250 - 253. https://doi.org/10.1002/ar.10037

Edwards, R. K., Kellner, K. R., Sistrom, C. L., \& Magyari, E. J. (2003). Medical student self-assessment of performance on an obstetrics and gynecology clerkship. American journal of obstetrics and gynecology, 188(4), 1078-1082. https://doi.org/10.1067/mob.2003.249

Efklides, A. (2011). Interactions of Metacognition With Motivation and Affect in Self- 
Regulated Learning: The MASRL Model. Educational Psychologist, 46, 25 - 6.

Estevez, M.E., Lindgren, K.A. and Bergethon, P.R. (2010), A novel three-dimensional tool for teaching human neuroanatomy. Anat Sci Ed, 3, 309

317. https://doi.org/10.1002/ase.186

Evenson, R. C., Holland, R. A., Mehta, S., \& Yasin, F. (1980). Factor analysis of the Symptom Checklist-90. Psychological reports, 46(3 Pt 1), 695-699. https://doi.org/10.2466/pr0.1980.46.3.695

Ferreri, F., Lapp, L.K., \& Peretti, C.S. (2011). Current research on cognitive aspects of anxiety disorders. Current Opinion in Psychiatry, 24(1), 4954. DOI: $10.1097 / \mathrm{YCO} .0 \mathrm{~b} 013 \mathrm{e} 32833 \mathrm{f5} 585$

Fillmore, E. and Helfenbein, R. (2015), Medical Student Grit and Performance in Gross Anatomy: What are the Relationships?. The FASEB Journal, 29: 689.6. https://doi.org/10.1096/fasebj.29.1_supplement.689.6

Fischl, B., Salat, D.H., Busa, E., Albert, M., Dieterich, M., Haselgrove, C., van der Kouwe, A., Killiany, R., Kennedy, D., Klaveness, S., Montillo, A., Makris, N., Rosen, B., \& Dale, A.M. (2002). Whole Brain Segmentation: Automated Labeling of Neuroanatomical Structures in the Human Brain. Neuron, 33(3), 341355. https://doi.org/10.1016/S0896-6273(02)00569-X.

Flanagan, E., Walsh, C. \& Tubridy, N.J. (2007). Neurophobia- attitudes of medical students and doctors in Ireland to neurological teaching. European Journal of Neurology, (14). DOI:10.1111/j.1468-1331.2007.01911.x

Flexner, A. (1908) The American college; a criticism. New York, The Century Co. [Web.] Retreived from the Library of Congress, https://lccn.loc.gov/08030713.

Flexner, A., Updike, D. B. (1910). Carnegie Foundation for the Advancement of Teaching, \& Merrymount Press. Medical education in the United States and Canada: A report to the Carnegie foundation for the advancement of teaching.

Flores, Andrew R., Jody L. Herman, Gary J. Gates, and Taylor N. T. Brown. (2016). How Many Adults Identify as Transgender in the United States? Los Angeles: The Williams Institute. Retrieved from: https://williamsinstitute.law.ucla.edu/publications/trans-adults-united-states/ [accessed 22 September 2019]

Foreman, K.B., Morton, D.A., Musolino, G.M., \& Albertine, K.H. (2005). Design and utility of a web-based computer-assisted instructional tool for neuroanatomy selfstudy and review for physical and occupational therapy graduate students. Anat Rec B New Anat, 285(1):26-31. DOI:10.1002/ar.b.20069 
Forester, J.P., McWhorter, D.L. \& Cole, M.S. (2002). The relationship between premedical coursework in gross anatomy and histology and medical school performance in gross anatomy and histology. Clin. Anat., 15, 160164. https://doi.org/10.1002/ca.1114

Francis, E.R., Bernard, S., Nowak, M. L., Daniel, S., \& Bernard, J.A. (2020). Operating Room Virtual Reality Immersion Improves Self-Efficacy Amongst Preclinical Physician Assistant Students. Journal of Surgical Education, 77(4), 947-952. https://doi.org/10.1016/j.jsurg.2020.02.013.

Fraser, G. (2018). Evaluating inclusive gender identity measures for use in quantitative psychological research. Psychology \& Sexuality, 9(4), 343-357. DOI: 10.1080/19419899.2018.1497693

Freeman, W. D., Vatz, K. A., Griggs, R. C., \& Pedley, T. (2013). The workforce task force report: Clinical implications for neurology. Neurology 81(5), 479-486.

Frenk, J., Chen, L., Bhutta, Z. A., Cohen, J. C., Crisp, N., Evans, T., Fineberg, H., Garcia, P., Ke, Y., Kelley, P., Kistmasamy B., Meleis, A., Naylor, D., Pablos-Mendez, A., Reddy, S., Scrimshaw, S., Sepulveda, J., Serwadda, D., \& Zurayl, H. (2010). Health professionals for a new century: transforming education to strengthen health systems in an interdependent world. Lancet, 376(9756), 1923-1958. DOI:10.1016/S0140-6736(10)61854-5

Garg, A., Serwint, J. R., Higman, S., Kanof, A., Schell, D., Colon, I., \& Butz, A. M. (2007). Self-efficacy for smoking cessation counseling parents in primary care: an office-based intervention for pediatricians and family physicians. Clinical pediatrics, 46(3), 252-257. https://doi.org/10.1177/0009922806290694

Giles, J. (2010). Clinical neuroscience attachments: a student's view of 'neurophobia'. The Clinical Teacher, 7, 9-13. https://doi.org/10.1111/j.1743-498X.2009.00330.x

Gonzalo, J.D., Dekhtyar, M., Starr, S.R., Borkan, J., Brunett, P., Fancher, T., Green, J., Grethlein, S.J., Lai, C., Lawson, L., Monrad, S., O'Sullivan, P., Schwartz, M.D., $\&$ Skochelak, S. (2017). Health Systems Science Curricula in Undergraduate Medical Education: Identifying and Defining a Potential Curricular Framework, Academic Medicine, 92(1),123-131. DOI: 10.1097/ACM.0000000000001177

Gottlieb-Smith, R., Balmer, D., \& Gelb, D. (2020). Why Neurology (or Not)? Understanding Career Decision-Making of First-Year Medical Students. Neurology, 94(15): 741.

Gould, D.J., Terrell, M.A., \& Fleming, J. (2008). A usability study of users' perceptions toward a multimedia computer-assisted learning tool for neuroanatomy. Anat Sci Educ, 1(4),175-183. DOI:10.1002/ase.36 
Greenwald, R., \& Quitadamo, I.J. (2014). A mind of their own: using inquiry-based teaching to build critical thinking skills and intellectual engagement in an undergraduate neuroanatomy course. Journal of undergraduate neuroscience education : JUNE : a publication of FUN, Faculty for Undergraduate Neuroscience, 12 2, A100-6.

Grupe, D. \& Nitschke, J. (2013). Uncertainty and anticipation in anxiety: an integrated neurobiological and psychological perspective. Nat Rev Neurosci, 14, 488-501. https://doi.org/10.1038/nrn3524

Gupta, N.B., Khadilkar, S.V., \& Bangar, S.S. (2013). Neurology as career option among postgraduate medical students. Ann Indian Acad Neurol, 16(4), 478-82.

Guttman, L. (1946). The test-retest reliability of qualitative data. Psychometrika, 11, 8195. https://doi.org/10.1007/BF02288925

Gutmann, L., Cahill, C., Jordan, J. T., Gamaldo, C. E., Santini, V., Ali, I., Soni, M.,Wilson, R. B., Said, R., Czeisler, B. M., \& Smith, A. G. (2019).

Characteristics of graduating US allopathic medical students pursuing a career in neurology. Neurology, 92(17), e2051-e2063. https://doi.org/10.1212/wnl.0000000000007369

Hall, S.R., Stephens, J.R., Seaby, E.G., Andrade, M.G., Lowry, A.F., Parton, W.J., Smith, C.F. \& Border, S. (2016). Can medical students accurately predict their learning? A study comparing perceived and actual performance in neuroanatomy. American Association of Anatomists, 9, 488-495. https://doi.org/10.1002/ase.1601

Hasan, T. F., Turnbull, M. T., Vatz, K. A., Robinson, M. T., Mauricio, E. A., \& Freeman, W. D. (2019). Burnout and attrition: Expanding the gender gap in neurology?. Neurology, 93(23), 1002-1008. https://doi.org/10.1212/WNL.0000000000008583.

Hayat, A. A., Kojuri, J., \& Amini, M. (2020). Academic procrastination of medical students: The role of Internet addiction. Journal of advances in medical education \& professionalism, $8(2), 83-89$. https://doi.org/10.30476/JAMP.2020.85000.1159.

Heisler, E.J., Jansen, D.J., Mitchell, A., Panangala, S.V., \& Villagrana, M.A. (2018). Congressional Research Service. Federal support for graduate medical education: an overview. https://fas.org/sgp/crs/misc/ R44376.pdf. [accessed 18 June 2020]

Hodgson, R., \& Rachman, S. (1974). II. Desyncnchrony in measures of fear. Behaviour Research and Therapy, 12(4), 319 - 326. DOI: https://doi.org/10.1016/00057967(74)90006-0

Hudson, J.N. (2006). Linking neuroscience theory to practice to help overcome student 
fear of neurology, Medical Teacher, 28:7, 651-

653, DOI: $10.1080 / 01421590600726409$

Hu ,Y., Brooks, K.D., Kim, H., Mahmutovic, A., Choi, J., Le, I.A., Kane, B.J., McGahren, E.D., \& Rasmussen, S.K. (2016). Adaptive simulation training using cumulative sum: a randomized prospective trial. Am J Surg, 211(2), 377-383. doi:10.1016/j.amjsurg.2015.08.030.

Irby, D. M., Cooke, M., \& O'Brien, B. C. (2010). Calls for reform of medical education by the Carnegie Foundation for the Advancement of Teaching: 1910 and 2010. Academic medicine : journal of the Association of American Medical Colleges, 85(2), 220-227. https://doi.org/10.1097/ACM.0b013e3181c88449.

Javaid, M. A., Chakraborty, S., Cryan, J.F., Schellekens, H., \& Toulouse, A. (2018). Understanding neurophobia: Reasons behind impaired understanding and learning of neuroanatomy in cross-disciplinary healthcare students. Anat Scie Educ, 11, 81-93.

Javaid, M. A., Chakraborty, S., Cryan, J.F., Schellekens, H., \& Toulouse, A. (2019). Evaluation of neuroanatomy web resources for undergraduate education: Educators' and students' perspectives. Anat Scie Educ, 13, 237-249.

Jena, A.B., Khullar, D., Ho, O., Olenski, A.R., \& Blumenthal, D.M. (2015). Sex Differences in Academic Rank in US Medical Schools in 2014. JAMA, 314(11), 1149-1158. DOI:10.1001/jama.2015.10680

Jena, A.B., Olenski, A.R., \& Blumenthal, D.M. (2016). Sex Differences in Physician Salary in US Public Medical Schools. JAMA Intern Med, 176(9), 1294-1304. DOI:10.1001/jamainternmed.2016.3284.

Jozefowicz, R.F. (1994). Neurophobia: The Fear of Neurology Among Medical Students. Archives of Neurology, 51(4), 328-329. doi:10.1001/archneur.1994.00540160018003

Jumat, M. R., Chow, P. K., Allen, J. C., Jr, Lai, S. H., Hwang, N. C., Iqbal, J., Mok, M., Rapisarda, A., Velkey, J. M., Engle, D. L., \& Compton, S. (2020). Grit protects medical students from burnout: a longitudinal study. BMC medical education, 20(1), 266. https://doi.org/10.1186/s12909-020-02187-1

Kaiser, H. F. (1991). Coefficient Alpha for a Principal Component and the KaiserGuttman Rule. Psychological Reports, 68(3), 855-858. https://doi.org/10.2466/pr0.1991.68.3.855

Kam, K. Q., Tan, G. S., Tan, K., Lim, E. C., Koh, N. Y., \& Tan, N. C. (2013). Neurophobia in medical students and junior doctors--blame the GIK. Annals of the Academy of Medicine, Singapore, 42(11), 559-566. 
Kirk, A., Meyer, J.M., Whisman, M.A., Deacon, B.J., \& Arch, J.J. (2019). Safety behaviors, experiential avoidance, and anxiety: a path analysis approach. Journal of Anxiety Disorders, 64, 9-15. https://doi.org/10.1016/j.janxdis.2019.03.002

Klassen, R.M., \& Klassen, J.R.L. (2018). Self-efficacy beliefs of medical students: a critical review. Perspect Med Educ, 7, 76-82. https://doi.org/10.1007/s40037018-0411-3

Klement, B.J., Paulsen, D.F., \& Wineski, L.E. (2011). Anatomy as the backbone of an integrated first year medical curriculum: design and implementation. Anat Sci Educ, 4, 157-69.

Kondrashov, P., McDaniel, D.J. \& Jordan, R.M. (2017). Premedical anatomy experience and student performance in medical gross anatomy. Clin. Anat, 30, 303-311. https://doi.org/10.1002/ca.22846.

Kockro, R.A., Amaxopoulou, C., Killeen, T., \& Wagner, W. (2015). Stereoscopic neuroanatomy lectures using a three-dimensional virtual reality environment. Annals of Anatomy, DOI: 10.1016/j.aanat.2015.05.006

Krasne, S., Hillman, J. D., Kellman, P. J., \& Drake, T. A. (2013). Applying perceptual and adaptive learning techniques for teaching introductory histopathology. Journal of pathology informatics, 4, 34. https://doi.org/10.4103/2153-3539.123991

Kroenke, K., Strine, T. W., Spitzer, R. L., Williams, J. B., Berry, J. T., \& Mokdad, A. H. (2009). The PHQ-8 as a measure of current depression in the general population. Journal of affective disorders, 114(1-3), 163-173. https://doi.org/10.1016/j.jad.2008.06.026

Kruger, R.A. (1994). Focus groups: a practical guide for applied research. 2nd Ed. Thousand Oaks, CA: Sage.

LaFaver, K., Miyasaki, J. M., Keran, C. M., Rheaume, C., Gulya, L., Levin, K. H., Jones, E. C., Schwarz, H. B., Molano, J. R., Hessler, A., Singhal, D., Shanafelt, T. D., Sloan, J. A., Novotny, P. J., Cascino, T. L., \& Busis, N. A. (2018). Age and sex differences in burnout, career satisfaction, and well-being in US neurologists. Neurology, 91(20), e1928-e1941. https://doi.org/10.1212/WNL.0000000000006497

Lang, P.J. (1971). The application of psychophysiological methods to the study of psychotherapy and behavior change. A.E. Bergin, S.L. Garfield (Eds.), Handbook of Psychotherapy and Behavior Change: An Empirical Analysis, Wiley, New York. 
Lang, P.J. (1978). Anxiety: Toward a psychophysiological definition. In: Akiskal HS, Webb WL, editor. Psychiatric Diagnosis: Exploration of Biological Predictors. New York: Spectrum, pp 365-389.

Lubelski, D., Xiao, R., Mukherjee, D., Ashley, W. W., Whitham, T., Brem, H., Huang, J., \& Wolfe, S. (2019). Improving medical student recruitment to neurosurgery, Journal of Neurosurgery, 1-7.

Lucey, C.R. (2013) Medical Education: Part of the Problem and Part of the Solution. JAMA Intern Med, 173(17), 1639-1643.

doi:10.1001/jamainternmed.2013.9074

Ludmerer, K. (1985). Learning to Heal: The Development of American Medical Education. New York, NY: Basic Books.

Ludmerer, K. (2010). Commentary: Understanding the Flexner report. Academicmedicine : journal of the Association of American Medical Colleges, 85(2), 193-196. https://doi.org/10.1097/ACM.0b013e3181c8fle7

Mabel, O. \& Olayemi, O. (2020). A comparison of principle component analysis, maximum likelihood and the principl axis in factor analysis. American journal of mathematics and statistics, 10(2): 44-54. DOI: 10.5923/j.ajms.20201002.03

Macchi, V., Porzionato, A., Stecco, C., Parenti, A., \& De Caro, R. (2007). Clinical neuroanatomy module 5 years' experience at the School of Medicine of Padova. Surg Radiol Anat, 29(3): 261-7. DOI: 10.1007/s00276-007-0201-9.

Martin, E. I., Ressler, K. J., Binder, E., \& Nemeroff, C. B. (2009). The neurobiology of anxiety disorders: brain imaging, genetics, and psychoneuroendocrinology. The Psychiatric clinics of North America, 32(3), 549-575. https://doi.org/10.1016/j.psc.2009.05.004

Mateen, F. J., \& D'Eon, M. F. (2008). Neuroanatomy: a single institution study of knowledge loss. Medical teacher, 30(5), 537-539. https://doi.org/10.1080/01421590802064880.

Matthias, A. T., Nagasingha, P., Ranasinghe, P., \& Gunatilake, S. B. (2013). Neurophobia among medical students and non-specialist doctors in Sri Lanka. BMC medical education, 13, 164. https://doi.org/10.1186/1472-6920-13164.

Mavis, B. (2001). Self-Efficacy and OSCE Performance Among Second Year Medical Students. Adv Health Sci Educ Theory Pract 6, 93-102. https://doi.org/10.1023/A:1011404132508

McBride, J.M. \& Drake, R.L. (2018). National survey on anatomical sciences in 
medical education. American Association of Anatomists, 11, 7-14.

DOI:10.1002/ase. 1760

McCarron, M.O., Stevenson, M., Loftus, A.M., \& McKeown, P. (2014). Neurophobia among general practice trainees: The evidence, perceived causes and solutions. Clin Neurol Neurosurg, 122, 124- 128.

McDermott, M., Gelb, D.J., Wilson, K., Pawloski, M., Burke, J.F., Shelgikar, A.V., \& London, Z.N. (2018). Sex Differences in Academic Rank and Publication Rate at Top-Ranked US Neurology Programs. JAMA Neurol, 75(8), 956-961. DOI:10.1001/jamaneurol.2018.0275

McKeown, P.P., Heylings, D.J., Stevenson, M., McKelvey, K.J., Nixon, J.R., \& McCluskey, D.R. (2003). The impact of curricular change on medical students' knowledge of anatomy. Med Educ, 37, 954-61.

Merkle, E. C., \& Van Zandt, T. (2006). An application of the Poisson race model to confidence calibration. Journal of Experimental Psychology: General, 135(3), 391-408. https://doi.org/10.1037/0096-3445.135.3.391

Michelson, L. (1986). Treatment consonance and response profiles in agoraphobia: the role of individual differences in cognitive, behavioral and physiological treatments. Behaviour research and therapy, 24(3), 263-275. https://doi.org/10.1016/0005-7967(86)90186-5

Miller-Matero, L. R., Martinez, S., MacLean, L., Yaremchuk, K., \& Ko, A. B. (2018). Grit: A predictor of medical student performance. Education for health (Abingdon, England), 31(2), 109-113. https://doi.org/10.4103/efh.EfH_152_16

Moreno-Zambrano, D., Meza, J., Freire-Bonifacini, A., Santana, D. , García-Santibáñez, R., \& Santibáñez-Vasquez, R. (2019). Neurophobia, a Worldwide Concern for Neurology Training: A Systematic Review. Neurology, 92 (15), 1.9-075.

Muller, J.H., Jain, S., Loeser, H. \& Irby, D.M. (2008), Lessons learned about integrating a medical school curriculum: perceptions of students, faculty and curriculum leaders. Medical Education, 42, 778-785. DOI:10.1111/j.13652923.2008.03110.x

Nagoshi, M. H., Tanabe M. K., Sakai, D. H., Masaki, K. H., Kasuya, R. T. \& Blanchette, P. L. (2008). The Impact of Curricular Changes on the Geriatrics Knowledge, Attitudes and Skills of Medical Students. Gerontology \& Geriatrics Education, 28(3), 47-58, DOI: 10.1300/J021v28n03_04

Ng-Sueng, L.F., Vargas-Matos, I., Mayta-Tristán, P., Pereyra-Elías, R., MontenegroIdrogo, J.J., Inga-Berrospi, F., ...Vidal, M. (2016). Gender Associated with the Intention to Choose a Medical Specialty in Medical Students: A Cross-Sectional 
Study in 11 Countries in Latin America. PLoS ONE, 11(8), e0161000. https://doi.org/10.1371/journal.pone.0161000

Nie, Y., Lau, S. \& Liau, A. (2011). Role of academic self-efficacy in moderating the relation between task importance and test anxiety. Learning and Individual Differences, 21, 736-741. DOI: 10.1016/j.lindif.2011.09.005.

Norton, G. R., \& Johnson, W. E. (1983). A comparison of two relaxation procedures for reducing cognitive and somatic anxiety. Journal of behavior therapy and experimental psychiatry, 14(3), 209-214. https://doi.org/10.1016/00057916(83)90050-2

O’Brien, B.C. \& Irby, D.M. (2013) Enacting the Carnegie Foundation Call for Reform of Medical School and Residency, Teaching and Learning in Medicine, 25, sup1, S1-S8, DOI: 10.1080/10401334.2013.842915.

Opacic, D.A. (2003). The relationship between self-efficacy and student physician assistant clinical performance. Journal of Allied Health, 32(3), 158-66. http://echo.louisville.edu/login?url=https://www.proquest.com/scholarlyjournals/relationship-between-self-efficacy-student/docview/210975759/se2? accountid=14665 [accessed 20 March 2021]

Ose, S.O. (2016). Using Excel and Word to Structure Qualitative Data. J Appl Soc Sci 10(2), 147-162. URL: https://doi.org/10.1177/1936724416664948

Öst, L., \& Hugdahl, K. (1981). Acquisition of phobias and anxiety response patterns in clinical patients. Behaviour Research and Therapy 19(5). 439-447. https://doi.org/10.1016/0005-7967(81)90134-0

Pajares, F. (1996). Self-Efficacy Beliefs in Academic Settings. Review of Educational Research, 66(4), 543-578. DOI:10.2307/1170653

Pakpoor, J., Handel, A. E., Disanto, G., Davenport, R. J., Giovannoni, G., \& Ramagopalan, S. V. (2014). National survey of UK medical students on the perception of neurology. BMC medical education, 14, 225. https://doi.org/10.1186/1472-6920-14-225

Peterson, C.A., \& Tucker, R.P. (2005). Undergraduate coursework in anatomy as a predictor of performance: Comparison between students taking a medical gross anatomy course of average length and a course shortened by curriculum reform. Clin. Anat., 18, 540-547. https://doi.org/10.1002/ca.20154

Pintrich, P. R., \& Schunk, D. (2002). Motivation in education: Theory, research, and applications (2nd ed.). Upper Saddle River, NJ: Prentice Hall.

Posner, J., Russell, J. A., \& Peterson, B. S. (2005). The circumplex model of affect: an 
integrative approach to affective neuroscience, cognitive development, and psychopathology. Development and psychopathology, 17(3), 715-734. https://doi.org/10.1017/S0954579405050340

Price, D. (2005). Continuing medical education, quality improvement, and organizational change: implications of recent theories for twenty-first-century CME. Med Teach, 27(3), 259-268. DOI:10.1080/01421590500046270

Rae, G., Cork, R.J., Karpinski, A.C., \& Swartz, W.J. (2016). The integration of brain dissection within the medical neuroscience laboratory enhances learning. American Association of Anatomists, 9, 565-574. https://doi.org/10.1002/ase.1611

Razavi, S. A., Shahrabi, A., \& Siamian, H. (2017). The Relationship Between Research Anxiety and Self-Efficacy. Materia socio-medica, 29(4), 247-250. https://doi.org/10.5455/msm.2017.29.247-250

Regenstein, M., Trott, J., Williamson, A., \& Theiss, J. (2018). Addressing Social Determinants Of Health Through Medical-Legal Partnerships. Health affairs (Project Hope), 37(3), 378-385. https://doi.org/10.1377/hlthaff.2017.1264.

Reisner, S.L., Conron, K.J., Tardiff, L.A., Jarvi, S., Gordon, A.R., \& Austin, S.B. (2014). Monitoring the health of transgender and other gender minority populations: validity of natal sex and gender identity survey items in a U.S. national cohort of young adults. BMC Public Health, 14, 1224. DOI: 10.1186/1471-2458-14-1224.

Romito, B. T., Krasne, S., Kellman, P. J., \& Dhillon, A. (2016). The impact of a perceptual and adaptive learning module on transoesophageal echocardiography interpretation by anaesthesiology residents, BJA: British Journal of Anaesthesia, 117(4), 477-481. https://doi.org/10.1093/bja/aew295

Roohi, G., Asayesh, H., Bathai, S. A., Shouri Bidgoli, A. R., Badeleh, M. T., \& Rahmani, H. (2013). The relationship between self-efficacy and academic motivation among students of medical sciences. Jmed, 8(1), 45-51. Retrieved March 5, 2021, from http://jmed.ssu.ac.ir/article-1-100-en.html

Rushmore, R.J., Bouix, S., Kubicki, M., Rathi, Y., Yeterian, E.H., \& Makris, N. (2020). How human is human connectional neuroanatomy? Frontiers in Neuroanatomy, 14 (18), 1 - 8. DOI: 10.3389/fnana.2020.00018

Russell, J. (1980). A Circumplex Model of Affect. Journal of Personality and Social Psychology, 39, 1161-1178. https://doi.org/10.1037/h0077714

Samulski, T.D., La, T., \& Wu, R.I. (2016). Adaptive eLearning modules for cytopathology education: A review and approach. Diagn Cytopathol, 44(11), 944951. DOI:10.1002/dc. 23558 
Samulski, T.D., Taylor, L.A., La, T., Mehr, C.R., McGrath, C.M., \& Wu, R.I. (2018). The utility of adaptive eLearning in cervical cytopathology education. Cancer cytopathology, 126(2), 129-135. https://doi.org/10.1002/cncy.21942

Sandrone, S., Chuquilin, M., Cios, J., Ghosh, P., Gottlieb-Smith, R., Kushlaf, H., Mantri, S., Masangkay, N., Menkes, D., Nevel, K., Sarva, H., \& Schneider, L. (2019). Neurologic and neuroscience education

Mitigating neurophobia to mentor health care providers. Neurology, 92(4), 174179; DOI: 10.1212/WNL.0000000000006716

Sanya, E.O., Ayodele, O.E., \& Olanrewaju, T.O. (2010). Interest in neurology during medical clerkship in three Nigerian medical schools. BMC Med Educ; 10, 36.

Saravanan C., \& Wilks, R. (2014). Medical students' experience of and reaction to stress: the role of depression and anxiety. The Scientific World Journal, 737382. DOI: $10.1155 / 2014 / 737382$.

Sawdon, M. \& Finn, G. (2014). The 'unskilled and unaware' effect is linear in a realworld setting. J. Anat., 224, 279-285. https://doi.org/10.1111/joa.12072

Schon ,F., Hart, P., \& Fernandez, C. (2002). Is clinical neurology really so difficult? Journal of Neurology, Neurosurgery \& Psychiatry, 72, 557-559. DOI: http://dx.doi.org/10.1136/jnnp.72.5.557

Schunk, D.H. (1991). Self-Efficacy and Academic Motivation. Educational Psychologist, 26, 3-4, 207-231, DOI: 10.1080/00461520.1991.9653133

Schunk, D. H., \& DiBenedetto, M. K. (2016). Self-Efficacy Theory in Education. Handbook of Motivation at School, 2, 34-54.

Selvarajah, L. \& Aojula, N. (2019). Decreasing teaching hours for undergraduate medical students: A step in the right direction?. Medical teacher, 1. https://doi.org/10.1080/0142159X.2019.1688274

Sethi, N.K. (2015). It is just not neurophobia! Editorial in: Neurology. American Academy of Neurology, Wolters Kluwer, Inc. URL: https://n.neurology.org/content/it-just-not-neurophobia [accessed 21 March 2020]

Shiels, L., Majmundar, P., Zywot, Sobotka, J., Lau, C., \& Jalonen, T.O. (2017). Medical student attitudes and educational interventions to prevent neurophobia: a longitudinal study. BMC Med Educ, 17, 225. https://doi.org/10.1186/s12909-0171055-4

Shin, L., \& Liberzon, I. (2010). The Neurocircuitry of Fear, Stress, and Anxiety Disorders. Neuropsychopharmacology, 35, 169-191. 
Shuck, B., Adelson, J.L., \& Reio, T.G., Jr. (2017). The Employee Engagement Scale: Initial Evidence for Construct Validity and Implications for Theory and Practice. Hum Resour Manage, 56, 953-977. https://doi.org/10.1002/hrm.21811

Siegel, M. G., Brand, J. C., Rossi, M. J., \& Lubowitz, J. H. (2018). "Publish or Perish" Promotes Medical Literature Quantity Over Quality. Arthroscopy : the journal of arthroscopic \& related surgery : official publication of the Arthroscopy Association of North America and the International Arthroscopy Association, 34(11), 2941-2942. https://doi.org/10.1016/j.arthro.2018.08.029

Skochelak S.E. (2010). A decade of reports calling for change in medical education: what do they say?. Academic medicine : Journal of the Association of American Medical Colleges, 85(9 Suppl), S26-S33. https://doi.org/10.1097/ACM.0b013e3181f1323f

Skochelak, S.E., \& Stack, S.J. (2017). Creating the Medical Schools of the Future. Academic medicine: Journal of the Association of American Medical Colleges, 92(1), 16-19. https://doi.org/10.1097/ACM.0000000000001160

Sotgiu, M.A., Mazzarello, V., Bandiera, P., Madeddu, R., Montella, A., \& Moxham, B. (2020). Neuroanatomy, the Achille's Heel of Medical Students. A Systematic Analysis of Educational Strategies for the Teaching of Neuroanatomy. Anatomical sciences education, 13(1), 107-116. https://doi.org/10.1002/ase.1866

Spielberger, C. D. (1989). State-Trait Anxiety Inventory: Bibliography (2nd ed.). Palo Alto, CA: Consulting Psychologists Press.

Spitzer, R. L., Kroenke, K., Williams, J. B., \& Löwe, B. (2006). A brief measure for assessing generalized anxiety disorder: the GAD-7. Archives of internal medicine, 166(10), 1092-1097. https://doi.org/10.1001/archinte.166.10.1092

Stegers-Jager, K.M., Cohen-Schotanus, J. \& Themmen, A.P.N. (2012). Motivation, learning strategies, participation and medical school performance. Medical Education, 46, 678-688. https://doi.org/10.1111/j.1365-2923.2012.04284.x

Strauss, A., \& Corbin, J. (1998). Basics of qualitative research: Techniques and procedures for developing grounded theory. $2^{\text {nd }}$ ed. Thousand Oaks, CA: Sage Publications, Inc. 336 p.

Svirko E., \& Mellanby, J. (2008). Attitudes to e-learning, learning style and achievement in learning neuroanatomy by medical students. Med Teach, 30(9-10), e219-e227. DOI:10.1080/01421590802334275

Svirko, E., \& Mellanby, J. (2017). Teaching neuroanatomy using computer-aided 
learning: What makes for successful outcomes? Anat Sci Educ, 10(6), 560-569. DOI:10.1002/ase. 1694

Tabachnick, B. G., \& Fidell, L. S. (2007). Using Multivariate Statistics (5th ed.). New York: Allyn and Bacon.

Tachere, R.O., \& Modirrousta, M. (2017). Beyond anxierty and agitation: a clinical approach to akathisia. The Royal Australian College of General Practitioners, 46(5), $296-298$.

Thompson, B. (2004). Exploratory and confirmatory factor analysis: Understanding concepts and applications. Washington, DC: American Psychological Association.

Thurmond, V.A. (2001). The Point of Triangulation. J Nurs Scholarsh, 33, 253-258.

Triola, M.M., Hawkins, R.E., \& Skochelak, S.E. (2018). The Time Is Now: Using Graduates' Practice Data to Drive Medical Education Reform, Academic Medicine, 93(6), 826-828. DOI: 10.1097/ACM.0000000000002176

Turan, S., Valcke, M., Aper, L., Koole, S., \& Derese, A. (2013). Studying self-efficacy beliefs in medical education. Procedia Social and Behavioral Sciences (pp. 13111314). Presented at the 3rd World Conference on Learning, Teaching and Educational Leadership (WCLTA), AMSTERDAM, NETHERLANDS: ELSEVIER SCIENCE BV.

United Nations. (2002). World Population Ageing, 1950-2050. Department of Economic and Social Affairs. Population Division. New York: United Nations. 483 p.

Ursua, F., Altholz, J., Durning, S., Torre, D., \& Dong, T. (2021). Once in the door, grit may matter more: an evaluation of grit in medical students. Military Medicine, 186(1-2), 13-17.

van Dalen, H.P. \& Henkens, K. (2012). Intended and unintended consequences of a publish-or-perish culture: A worldwide survey. J Am Soc Inf Sci Tec, 63, 1282-1 293. DOI:10.1002/asi.22636

van Dinther, M., Dochy, F., \& Segers, M. (2011). Factors affecting students' selfefficacy in higher education. Educational Research Review 6(2), 95 - 108. https://doi.org/10.1016/j.edurev.2010.10.003

Veeramani, R., Madhugiri, V.S., \& Chand, P. (2015). Perception of MBBS students to "flipped class room" approach in neuroanatomy module. Anatomy \& Cell Biology 48(2),138-143. DOI: 10.5115/acb.2015.48.2.138

Whissell, C.M. (1989). The dictionary of affect in language. In R. Plutchik \& H. 
Kellerman (Eds.), The Measurement of Emotions (pp. 113-131). Academic Press. DOI: 10.1016/B978-0-12-558704-4.50011-6

Whissell, C. (2009). Using the Revised Dictionary of Affect in Language to quantify the emotional undertones of samples of natural language. Psychological reports, 105(2), 509-521. https://doi.org/10.2466/PR0.105.2.509-521

World Health Organization. (2008). Neurological disorders: Public health challenges. $J$ Nerv Ment Dis 196(2): 176.

Wu, H., Li, S., Zheng, J., \& Guo, J. (2020). Medical students' motivation and academic performance: the mediating roles of self-efficacy and learning engagement. Medical education online, 25(1), 1742964. https://doi.org/10.1080/10872981.2020.1742964

Young, H. N., Schumacher, J. B., Moreno, M. A., Brown, R. L., Sigrest, T. D., McIntosh, G. K., Schumacher, D. J., Kelly, M. M., \& Cox, E. D. (2012). Medical student self-efficacy with family-centered care during bedside rounds. Academic medicine : journal of the Association of American Medical Colleges, 87(6), 767-775. https://doi.org/10.1097/ACM.0b013e318253dcdb

Youssef, Y. (2009). Neurophobia and Its Implications: Evidence from a Caribbean Medical School. BMC Education, 9(39), DOI:10.1186/1472-6920-9-39.

Zagni, E., Simoni, L., \& Colombo, D. (2016). Sex and Gender Differences in Central Nervous System-Related Disorders. Neuroscience journal, 2827090. https://doi.org/10.1155/2016/2827090.

Zinchuk, A.V., Flanagan, E.P., Tubridy, N.J., Miller, W.A. \& McCullough, L.D. (2010). Attitudes of US medical trainees towards neurology education: Neurophobia- a global issue. BMC Medical Education, 10(49). DOI: 10.1186/1472-6920-10-49. 


\section{APPENDICES}

\section{APPENDIX A}

List of abbreviations

AAMC - American Association of Medical Colleges

AAN - American Academy of Neurologists

AERA - American Educational Research Association

AMA - American Medical Association

ANCOVA - Analysis of Covariance

ANOVA - Analysis of Variance

AP - Anatomy \& Physiology (premedical experience secondary to a course in Chapter 4)

APA - American Psychological Association

BAI- Beck Anxiety Inventory

BCPM GPA - Biochemistry, Chemistry, Physics, and Mathematics Grade Point Average

CBT-Case-based Teaching

CADE - Clinical Anatomy, Development and Examination

CME - Council on Medical Education

CST - Corticospinal Tract

DCML - Dorsal Column Medial Lemniscus

DAL - Dictionary of Affect in Language

DSM-5 - Diagnostic and Statistical Manual of Mental Disorders, $5^{\text {th }}$ edition

EFA - Exploratory Factor Analysis

EPC - Educational Program Committee 
GAD-7 - Generalized Anxiety Disorder Scale

GDTF - Gender Disparity Task Force

GPA - Grade Point Average

GR - Gross Anatomy (premedical experience secondary to a course in Chapter 4)

HPA- Hypothalamic Pituitary Adrenal Axis

JAMA - Journal of American Medical Association

KMO - Kaiser-Meyer-Olkin

LMN - Lower Motor Neuron

LMS - Learning Management System

M1- First-year Medical Student

M2 - M4 - Upper-class Medical Student (Second, Third, or Fourth Year)

MCAT - Medical College Admissions Test

mPFC - Medial Prefrontal Cortex

NA - Neuroanxiety

NAC - Neuroanatomy Course

NAS - Neuroanatomy Anxiety Scale

NBME - National Board of Medical Examiners

NCME - National Council on Measurement in Education

$\mathrm{NE}$ - Neuroscience and/or Neuroanatomy Experience

$\mathrm{NIH}$ - National Institute of Health

NPN - No Premedical Neuroscience or Neuroanatomy

NSAC - Neuroscience and Neuroanatomy Course

NSC - Neuroscience Course 
NSE - Neuroanatomy Self-Efficacy

NSES - Neuroanatomy Self-Efficacy Scale

OSCE - Objective Structured Clinical Examination

PBL - Problem Based Learning

PAF - Principal Axis Factoring

PCA- Principal Component Analysis

PHQ-8 - Patient Health Questionnaire

SC-90 - Symptom Checklist-90

SCT - Social Cognitive Theory

STAI- State Trait Anxiety Inventory

STT - Spinothalamic Tract

TBL - Team Based Learning

TOE - Transesophageal Echocardiography

UAMA - Uncertainty and Anticipation Model of Anxiety

UME - Undergraduate Medical Education

UMN - Upper Motor Neuron

UN - United Nations

USMLE - United States Medical Licensing Examination ${ }^{\circledR}$

ULSOM - University of Louisville School of Medicine

WHO - World Health Organization 


\section{APPENDIX B}

Scales

\section{NEUROANATOMY SELF-EFFICACY SCALE (NSES)}

\section{Please CIRCLE the number that applies}

1 - If you are not at all confident that you can do the task

2 - If you are only a little confident that you can do the task

3 - If you are fairly confident that you can do the task

4 - If you are very confident that you can do the task

5 - If you are extremely confident that you can do the task

\begin{tabular}{|c|c|c|c|c|c|}
\hline & $\begin{array}{l}\text { Not at } \\
\text { all confi- } \\
\text { dent }\end{array}$ & $\begin{array}{l}\text { Only a } \\
\text { little } \\
\text { confi- } \\
\text { dent }\end{array}$ & $\begin{array}{l}\text { Fairly } \\
\text { confi- } \\
\text { dent }\end{array}$ & $\begin{array}{l}\text { Very } \\
\text { confi- } \\
\text { dent }\end{array}$ & $\begin{array}{l}\text { Extremely } \\
\text { confident }\end{array}$ \\
\hline $\begin{array}{l}\text { 1. I am confident that I can identify brain } \\
\text { structures }\end{array}$ & 1 & 2 & 3 & 4 & 5 \\
\hline $\begin{array}{l}\text { 2. I am confident that I can identify the } \\
\text { orientation of brain slices (example: sagittal, } \\
\text { horizontal, coronal) }\end{array}$ & 1 & 2 & 3 & 4 & 5 \\
\hline $\begin{array}{l}\text { 3. I am confident that I can correctly pronounce } \\
\text { neuroanatomical terms }\end{array}$ & 1 & 2 & 3 & 4 & 5 \\
\hline $\begin{array}{l}\text { 4. I am confident that I can learn neuroanatomy } \\
\text { relationships (i.e. how one item relates to } \\
\text { another in position in the brain) }\end{array}$ & 1 & 2 & 3 & 4 & 5 \\
\hline $\begin{array}{l}\text { 5. I am confident that I can learn } \\
\text { neuroanatomical terms and definitions }\end{array}$ & 1 & 2 & 3 & 4 & 5 \\
\hline $\begin{array}{l}\text { 6. I am confident that I can perform successfully } \\
\text { on the neuroanatomy course written exams }\end{array}$ & 1 & 2 & 3 & 4 & 5 \\
\hline $\begin{array}{l}\text { 7. I am confident that I can perform successfully } \\
\text { on the neuroanatomy lab practical exams }\end{array}$ & 1 & 2 & 3 & 4 & 5 \\
\hline $\begin{array}{l}\text { 8. I am confident that I will be able to retain and } \\
\text { recall neuroanatomical knowledge for use in a } \\
\text { clinical setting }\end{array}$ & 1 & 2 & 3 & 4 & 5 \\
\hline $\begin{array}{l}\text { 9. I am confident that I can actively participate } \\
\text { in neuroanatomical discussions }\end{array}$ & 1 & 2 & 3 & 4 & 5 \\
\hline $\begin{array}{l}10 \text {. I am confident that I can locate clinically } \\
\text { relevant neuroanatomical lesions in the human } \\
\text { brain }\end{array}$ & 1 & 2 & 3 & 4 & 5 \\
\hline $\begin{array}{l}\text { 11. I am confident that I can describe } \\
\text { neuroanatomical structures of the brain to a non- } \\
\text { medical person }\end{array}$ & 1 & 2 & 3 & 4 & 5 \\
\hline $\begin{array}{l}\text { 12. I am confident that I can successfully answer } \\
\text { neuroanatomically-based questions during } \\
\text { clinical rotations }\end{array}$ & 1 & 2 & 3 & 4 & 5 \\
\hline $\begin{array}{l}\text { 13. I am confident that I learned the } \\
\text { neuroanatomical content of this course }\end{array}$ & 1 & 2 & 3 & 4 & 5 \\
\hline
\end{tabular}

PREMEDICAL NEUROSCIENCE EXPERIENCE SCALE 


\section{For purposes of this survey, please review the following definitions:}

- Neuroanatomy: Anatomical connectivity within the brain and spinal cord (specific tracts such as the corticospinal tract, spinocerebellar tract, etc...), how these tracts function and isolating the location of lesions utilizing clinical scenarios.

- Neuroscience: The study of neurons, synapses and neuronal connectivity at the molecular level.

1. Have you ever been enrolled in a neuroanatomy (brain and/or spinal cord anatomy) course before starting medical school?
○ Yes
- No

If yes, what was the grade you received in the course?
○ A $(90-100 \%)$
B $(80-89 \%)$
$\mathrm{C}(70-79 \%)$
$\mathrm{D}(60-69 \%)$
F $(59 \%$ or below)
I do not recall my grade in the course, but I passed the course
I dropped the course
- Other Circumstance:

2. Have you ever been enrolled in a college-level neuroscience (molecular study) course before starting medical school?
○ Yes
○ No

If yes, what was the grade you received in the course?
○ A $(90-100 \%)$
○ $\mathrm{B}(80-89 \%)$
○ $\mathrm{C}(70-79 \%)$
○ $\mathrm{D}(60-69 \%)$
○ F (59\% or below)
- I do not recall my grade in the course, but I passed the course
- I dropped the course
- Other Circumstance:

3. I studied neuroanatomy as part of a gross anatomy course before starting medical school
○ Yes
$\circ$ No

4. I studied neuroanatomy as part of an anatomy and physiology course before starting medical school
○ Yes
$\circ$ No

5. I studied neuroanatomy in any capacity before starting medical school
$\circ$ Yes
$\circ$ No

If yes, please briefly explain the capacity in which you studied neuroanatomy:

$\circ$ None of these apply and I have never studied neuroscience or neuroanatomy on my own or worked with neuroscience before starting medical school. 


\section{NEUROANXIETY SCALE (NAS)}

Please CIRCLE the number the applies:

While studying neuroanatomy, during neuroanatomy lectures or during a neurological clinical setting, how often have you been bothered by the following?

$0=$ Not at all sure

$1=$ Several Days

2=Over Half the days

3=Nearly Every Day

\begin{tabular}{|l|c|c|c|c|}
\hline & $\begin{array}{c}\text { Not at } \\
\text { all }\end{array}$ & $\begin{array}{c}\text { Several } \\
\text { Days }\end{array}$ & $\begin{array}{c}\text { Over } \\
\text { Half the } \\
\text { Days }\end{array}$ & $\begin{array}{c}\text { Nearly } \\
\text { Every } \\
\text { Day }\end{array}$ \\
\hline 7. Feeling nervous, anxious or on edge & 0 & 1 & 2 & 3 \\
\hline 8. Not being able to stop or control thinking neuroanatomy is too difficult & 0 & 1 & 2 & 3 \\
\hline 9. Worrying too much and not being able to focus on neuroanatomy & 0 & 1 & 2 & 3 \\
\hline 10. Trouble completing lesion isolation case scenario or clinical problems & 0 & 1 & 2 & 3 \\
\hline 11. Being so restless that it's hard to sit still & 0 & 1 & 2 & 3 \\
\hline 12. Becoming easily annoyed or irritable & 0 & 1 & 2 & 3 \\
\hline $\begin{array}{l}\text { 13. Feeling afraid that I either won't pass neuroanatomy or properly } \\
\text { diagnose a neurology patient }\end{array}$ & 0 & 1 & 2 & 3 \\
\hline
\end{tabular}




\section{NAS SCALE MODIFICATIONS FROM THE GAD-7}

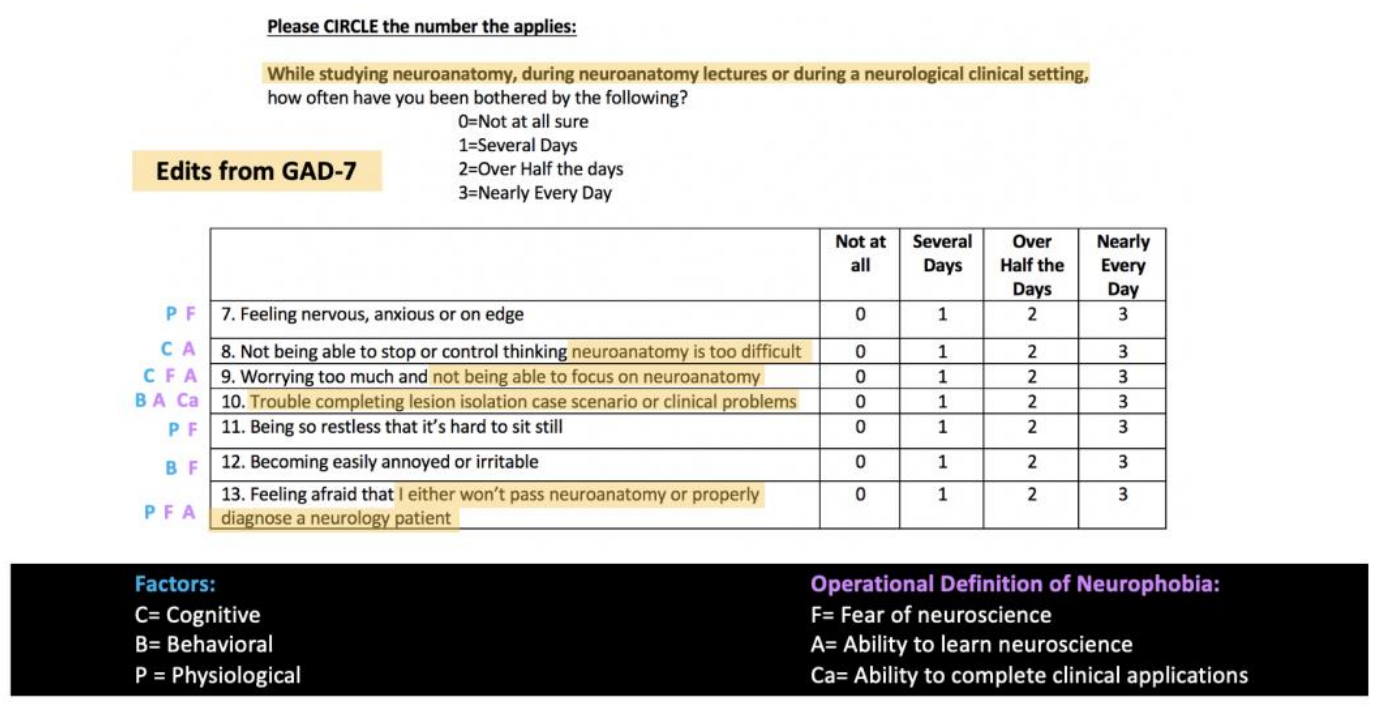

The highlighted portions of the text are wording edits from the GAD-7 Scale (Spitzer et al., 2006) to reflect anxiety specific to learning and applying neuroanatomy. Dimensions represent how each of the items reflect the three dimensions of anxiety related to learning neuroanatomy: Items 2 and 3 reflect cognitive aspects of anxiety, items 4 and 6 reflect the behavioral ramifications of anxiety, and items 1,5 , and 7 reflect physiological symptoms of anxiety. The operational definition of neuroanxiety shows how each item relates to measuring each aspect of neuroanxiety: Items $1,3,5$, and 6 relates to fear of neuroscience; items 2, 3, 4, and 7 relate to the ability to learn neuroscience; and item 4 relates to the ability to apply neuroanatomy to a clinical setting. 


\author{
CURRICULUM VITAE \\ Jessica S. Bergden, M.S., D.C.
}

\title{
EDUCATION:
}

Ph.D.

University of Louisville, Louisville, KY

2021 (Expected), Anatomical Sciences and Neurobiology Advisor: Jennifer Brueckner-Collins, Ph.D. Specialization: Medical Education

M.S.

University of Colorado Anschutz Medical Campus, Aurora, CO 2015, Modern Human Anatomy Advisor: W. Frank Hughes, Ph.D. Specialization: Neuroanatomy eLearning

D.C. Palmer College of Chiropractic, Davenport, IA 2001, Doctor of Chiropractic

B.S. Purdue University, West Lafayette, IN 1996, Biology

\section{ACADEMIC POSITIONS:}

Teaching Assistant University of Louisville, Louisville, KY $2017-2020$

Adjunct Professor Rocky Vista University, Parker, CO 2016

Teaching Assistant University of Colorado Anschutz Medical Campus, Aurora, CO $2014-2015$

Adjunct Professor Middlesex Community College, Bedford, MA $2010-2013$

\section{AWARDS:}

Ruth Greenberg Award in Medical Education Research, 2 ${ }^{\text {nd }}$ Place at Research!Louisville 2018 


\section{PROFESSIONAL ACTIVITIES:}

U of L Academic Technology Support Committee Graduate Student Representative 2018-2020

Louisville Student Chapter for the Society for Neuroscience Member 2017-2020

American Association of Anatomists Member 2019-2020

U of L Delphi University Participant 2017

Society for Neuroscience Member 2015

Women in Wellness Co-Founder 2004

Business Networking International Visitor Host 2003 - 2004

Warwick Rotary Chair of Charity Committee, Board of Directors 2002 - 2005

Big Sister Fund Board of Directors $2002-2004$

Leadership Warwick Participant 2001

NCCJ Wall of Hope Project Ambassador 2001 - 2002

Rhode Island Chiropractic Alliance Member 2001 - 2007

\section{TEACHING:}

University of Louisville School of Medicine, Louisville, KY

Gross Anatomy for Massage Therapy Students*

Dissection Instructor

ASNB 801/601 Medical Gross Anatomy

Lecturer \& Laboratory Instructor

2017- 2020

BMSC 809/ASNB 672 Dental Gross Anatomy

Lecturer \& Laboratory Instructor

$2018-2021$

Human Anatomy Neurobiology Degree Students

Mentor

$2017-2021$

Distinction in Medical Education Track for Medical Students

Mentor

$2019-2021$

Paige Hart (Class 2021): Development of a competency-based neuroanatomy eLearning intervention designed to bridge a gap within an integrated curriculum 
Rocky Vista University, Parker, CO

Human Gross Anatomy: Osteopathic Medicine Students

Lab Instructor

2016

Neuroanatomy: Osteopathic Medicine Students

Lab Instructor

2016

University of Colorado Anschutz Medical Campus, Aurora, CO

Human Body Block: Medical Gross Anatomy

Lab Instructor

2014

ANAT 6111 Gross Anatomy: Graduate Students

Lecturer \& Lab Instructor

2015

Physical Medicine \& Rehabilitation Residency

Prosection Demonstrations

2015

Middlesex Community College Bedford, MA \& Lowell, MA

BIO 231 Anatomy and Physiology I*

BIO 232 Anatomy and Physiology II*

BIO 131 General Biology I*

BIO 120 Introduction to Biology*

BIO 105 Basic Anatomy and Physiology*
Lecturer \& Lab Instructor

Lecturer \& Lab Instructor

Lecturer \& Lab Instructor

Lecturer \& Lab Instructor

Lecturer
$2010-2013$

$2010-2013$

$2010-2013$

$2010-2013$

$2010-2013$

*Denotes developed course

\section{MULTIMEDIA EDUCATIONAL PROGRAM DEVELOPMENT:}

Each of the following programs were authored in Articulate Storyline ${ }^{\circledR}$. Each program is an individually published, web-based multimedia educational module with interactive features such as click-to-identify and drag-and-drop images, instructional videos, and competency based quiz questions.

Bergden, J. (2018, July). Neuroanatomy Pathways. Interactive, step-by-step approach to teaching the three main neuroanatomy tracts: corticospinal, dorsal column-medial lemniscus, and spinothalamic. Designed to specifically reduce neuroanatomy anxiety and improve neuroanatomy self-efficacy in first year medical students. Administered in the Fall of 2018 at the University of Louisville School of Medicine, Louisville, KY. (Articulate Storyline)

Bergden, J. (2018, August). Orientation to Brain Sectional Anatomy. A basic orientation to neuroanatomy cross-sectional anatomy for first year medical students. Designed to guide novice learners to develop pattern recognitions skills to more quickly and easily identify coronal, horizontal and sagittal images of the brain. Administered during Fall Semesters 2018 - 2020 at the University of Louisville School of Medicine, Louisville, KY. 
Bergden, J. (2018, September). Brainstem Level Identification. A first-year medical student introduction to identifying levels of the brainstem. This program includes three main sections: (1) draw-it-to-learn-it guided video to drawing midbrain, pons, and medulla cross-sections; (2) pattern recognition guidance for novice neuroanatomy learners to recognize differences between cranial and caudal midbrain, pons, and medulla cross-sections; and (3) a practice drag-and-drop style quiz to help students orient the 2D cross-sectional brainstem images to their corresponding location on a 3D image. Administered during Fall Semesters 2018 - 2020 at the University of Louisville School of Medicine, Louisville, KY.

Bergden, J. (2018, November). Larynx eLearning Flipped Classroom. A first-year medical student model for a flipped classroom. Students are guided through lecture content in sections from the basic overview of structure and function, laryngeal skeleton, musculature, and corresponding vascular supply and nerve innervation. Each section consists of a short instructional video and an interactive competency based quiz to master before moving on to the next section. Administered during Fall Semesters 2018 - 2020 at the University of Louisville School of Medicine, Louisville, KY.

Bergden, J. (2019, July). Fun with Spinal Cord Lesions. A practical guide to assist firstyear medical students with the thought process necessary to identify spinal cord lesions questions using clinical cases. A draw-it-to-learn-it video shows students how to quickly draw the three major tracts (corticospinal, dorsal column-medical lemniscus, and spinothalamic) while demonstrating how to think through, draw, and solve neuroanatomy lesion problems during a high-stakes exam situation. A review quiz at the end of the module ensures competency. Administered during Fall Semesters 2019 - 2020 at the University of Louisville School of Medicine, Louisville, KY.

Bergden, J. (2019, August). Adaptive Neuroanatomy Pathways. A redesign of the original interactive, step-by-step approach to teaching the three main neuroanatomy tracts: corticospinal, dorsal column-medial lemniscus, and spinothalamic to first-year medical students. This redesign includes: competency based checkpoints, remediation loops with further instructional videos, and reassessments to ensure competency. Designed to target neuroanatomy anxiety and improve neuroanatomy self-efficacy in first year medical students. Administered during Fall Semesters 2019 - 2020 at the University of Louisville School of Medicine, Louisville, KY.

Bergden, J. (2019, September). Basal Ganglia Primer. This short module is designed as a primer for first-year medical students to work through prior to delivery of the lecture content. A review of the neuroanatomy structures that comprise the basal ganglia, neurotransmitters involved, and direct and indirect pathways is provided. Administered during Fall Semesters 2019 - 2020 at the University of Louisville School of Medicine, Louisville, KY.

Bergden, J. (2019, February). PGY1 Neurology Resident Education. Three independent modules were designed for PGY1 neurology residents: Somatomotor Neuroanatomy Pathways, Somatosensory Neuroanatomy Pathways, and Blood Supply Review. 
Additional content was added to the first-year medical student Neuroanatomy Pathways modules: extrapyramidal and corticobulbar tracts with corresponding clinical lesion case example quiz questions. A novel Blood Supply Review module was designed for neurology residents with interactive videos from the Cerefy Atlas of Cerebral Vasculature $^{\circledR}$. Major segments and subsequent branches of each cerebral artery were reviewed. Vascular supply to the brainstem was reviewed along with clinical-case quiz questions for learners to identify the vascular lesion location if provided either the affected brainstem area or clinical deficits. Embedded quiz questions throughout the program ensure competency. Administered during the Spring 2019 Semester at the University of Louisville School of Medicine, Louisville, KY.

Bergden, J. (2019, February). Neuroanatomy Bridging Activity. A review of major neuroanatomy content for second-year medical students. This module was designed to bridge a one-year time gap between the delivery of the first-year neuroanatomy content and the start of their second-year Neuropathology course. This module is comprised of content adapted from: Neuroanatomy Pathways, Brainstem Level Identification, Basal Ganglia Primer, and the neurology resident Blood Supply Review programs. It also provides a novel case review where students are provided video examples of case history and neurological examinations of mock patients. Students must make diagnostic decisions after visualizing these examinations and are guided towards narrowing down their differential diagnoses as they work through the case. Embedded quiz questions throughout the program ensure competency. Administered during Spring Semesters 2019 - 2020 at the University of Louisville School of Medicine, Louisville, KY.

\section{POSTER PRESENTATIONS:}

Bergden, J., Immekus, J., Sawning, S., Carr, E., \& Brueckner-Colllins, J. (2020, April). Neurophobia: Anxiety and Self-Efficacy Related to Learning Neuroanatomy in a Medical Student Population. Poster session to be presented at the Annual Meeting at Experimental Biology, San Diego, CA. *Meeting cancelled due to COVID-19

Bergden, J., Immekus, J., Sawning, S., Carr, E., \& Brueckner-Colllins, J. (2019, October). Neurophobia: Anxiety and self-efficacy related to learning neuroanatomy among neurology residents and medical students. Poster session presented at the annual Research!Louisville meeting, Louisville, KY.

Bergden, J., Noonan, E., Maglinger, G. B., Sawning, S., Carr, E., \& Brueckner-Colllins, J. (2019, April). Investigating causes of neurophobia to inform the development of an eLearning intervention in a first-year medical student population. Poster session presented at the Association of American Medical Colleges Central and Southern GSA/OSR Joint Regional Spring Meeting, Louisville, KY.

Bergden, J., Immekus, J., Sawning, S., Carr, E., \& Brueckner-Colllins, J. (2019, March). Neurophobia: Gender-Specific Differences in Anxiety and Self-Efficacy Related to Learning Neuroanatomy in a Medical Student Population. Poster session presented at the Human Anatomy and Physiological Society Regional Meeting, Bellarmine University, Louisville, KY. 
Bergden, J., Immekus, J., Sawning, S., Carr, E., \& Brueckner-Colllins, J. (2018, October). Investigating causes of neurophobia in the first-year medical student population. Poster session presented at the annual Research!Louisville meeting, Louisville, KY.

Bergden, J., Stabio, M. E. (2016, April). Development of a 3D Interactive Model for Teaching Intercortical Connectivity of Cerebral White Matter Tracts. Poster session presented at the Annual Meeting at Experimental Biology, San Diego, CA.

Bergden, J., Hughes, W. F., \& Caldwell, J. (2015, October). Dementia in 3D: A casebased instructional module exploring the impact of cerebral vascular anatomy in subcortical pathology leading to dementia. Poster session presented at the annual Society for Neuroscience Conference, Chicago, IL.

\section{INVITED PRESENTATIONS:}

Bergden, J. (2020, August). Using Student Feedback for Quality Improvement in Basic Sciences. Presentation at the Medical Education Grand Rounds in the Faculty Development Series, University of Louisville, Louisville, KY.

Bergden, J. (2019, February). Neurophobia: Investigating causes, effects and interventions at the University of Louisville. Presentation at the Seminars in Anatomical Sciences and Neurobiology, University of Louisville, Louisville, KY.

Bergden, J. (2019, February). NAS7: Neuroanxiety Scale Development and Initial Data. Presentation to ECPY 740 Instrument Development course at University of Louisville, Louisville, KY.

Bergden, J. (2019, October). Road to a PhD in Anatomical Sciences and Neurobiology. Presentation to the Future Physicians of America Club, Male High School, Louisville, KY.

\section{WORKSHOPS:}

Bergden, J., Brueckner-Collins, J., Sharp, M. Herring, N. (2019, March). Surgical Dissections as an Instructional Tool. Workshop conducted at the Human Anatomy and Physiology Society Regional Meeting, Bellarmine University, Louisville, KY.

\section{PUBLICATIONS:}

Cardenas-Trowers O.O., Bergden J.S., Gaskins J.T., Gupta A.S., Francis S.L., Herring N.R. (2020). Development of a safety zone for rectus abdominis fascia graft harvest based on dissections of the ilioinguinal and iliohypogastric nerves. Am J ObstetGynecol, 222:480.e1-7.

\section{SERVICE:}


Brain Days, Comparative Anatomy, Kentucky Science Center Presenter

exBEERiment Fundraiser, Kentucky Science Center Volunteer

Forensic Comparative Anatomy, Kentucky Science Center Co-Presenter

High School Science Fair, U of L Poster Judge

Academic Technology Support Committee, U of L Student Representative

$2017-2019$

\section{CLINICAL EXPERIENCE:}

Bergden Chiropractic, Chiropractor

2003- 2008

Warren Chiropractic, Associate Chiropractor

$2001-2002$

PROFESSIONAL LICENSURE:

RI Department of Health

$2001-2008$

National Board of Chiropractic Examiners, Parts I - IV

2001 